Copyright: The author, John Godwin, 2020.

\title{
A Mathematical Study of Amplitudes Through Their Singularities
}

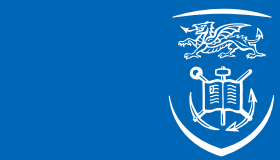

Swansea University

Prifysgol Abertawe

John H. Godwin

Department of Physics

Swansea University

Submitted to Swansea University in fulfilment of the requirements for the degree of

Doctor of Philosophy 


\begin{abstract}
In this thesis, amplitudes in pure Yang-Mills and an extended theory of gravity are investigated through the use of four-dimensional generalised unitarity and recursive techniques. In particular, the two-loop all-plus helicity amplitude in Yang-Mills is examined which, when viewed through the lens of four-dimensional unitarity, has a singularity structure related to general one-loop amplitudes. This helicity amplitude is computed for $n=6,7$ at leading order in the number of colours, presenting new results for the rational terms of these amplitudes through augmented recursion. Augmented recursion deals with the complications faced when performing complex recursion on an amplitude that contains double poles in particular momentum channels. A section is dedicated to reviewing this method in great detail. Additionally, the reach of four-dimensional unitarity and recursion is extended to all orders in the number of colours, and used to compute all partial amplitudes that contribute to the two-loop $n=5$ all-plus amplitude in $U\left(N_{c}\right)$ Yang-Mills. In the second half of the thesis, tree-level and one-loop amplitudes that arise in a theory of EinsteinHilbert (EH) gravity extended by an $\alpha R^{3}$ term are examined. Such a theory provides counterterms for two-loop amplitudes in EH gravity. Focussing on the leading deformation of $\mathrm{EH}$ gravity, the $\mathcal{O}(\alpha)$ treelevel $S$-Matrix is obtained solely by demanding the amplitudes factorise as a propagator goes on shell, and defining the $\mathcal{O}\left(\alpha^{0}\right)$ and $\mathcal{O}(\alpha)$ three-point amplitudes. Finally, the renormalisability of the one-loop amplitudes in this theory are studied by using four-dimensional techniques to determine the UV singularities present in bubble integral functions.
\end{abstract}




\section{Declaration}

This work has not previously been accepted in substance for any degree and is not being concurrently submitted for any degree.

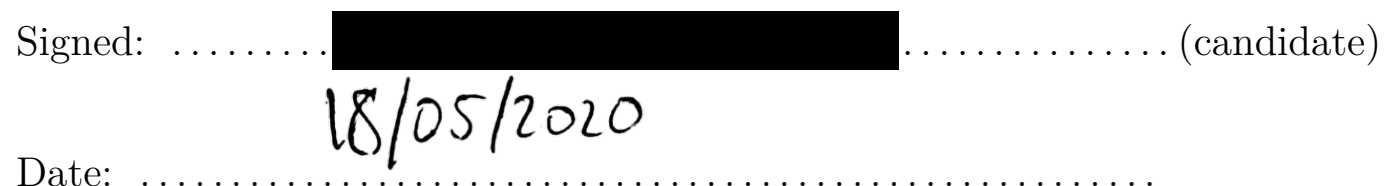

\section{Statement 1}

This thesis is the result of my own investigations, except where otherwise stated. Where correction services have been used, the extent and nature of the correction is clearly marked in a footnote(s). Other sources are acknowledged by footnotes giving explicit references. A bibliography is appended.

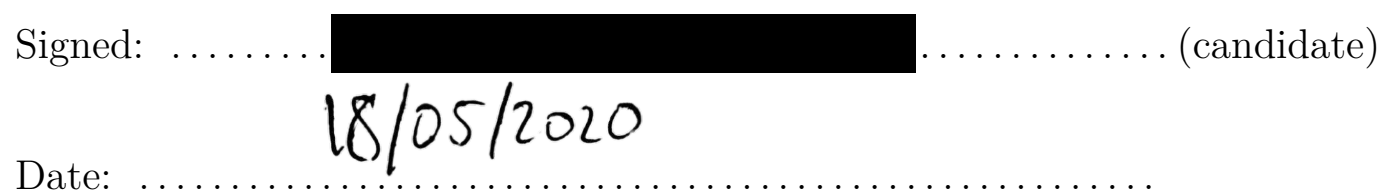

\section{Statement 2}

I hereby give consent for my thesis, if accepted, to be available for photocopying and for inter-library loan, and for the title and summary to be made available to outside organisations.

Signed:

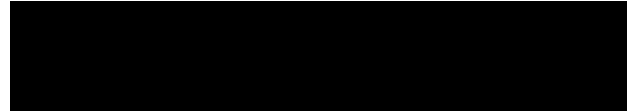
(candidate)

Date:

$$
18 / 05 / 2020
$$




\section{Contents}

List of Figures ix

1 Introduction and Historical Overview 1

2 Review of Techniques for Calculating Scattering Amplitudes 15

2.1 Pure Yang-Mills and Pertubative Gravity . . . . . . . . . . . . 18

2.1.1 Pure Yang-Mills . . . . . . . . . . . . . . . 18

2.1.2 Pertubative Gravity . . . . . . . . . . . . . . 21

2.2 Perturbation Theory and Conventions . . . . . . . . . . . 22

2.3 Colour Ordering and Group Theory Relations . . . . . . . . . . . 24

2.3.1 Colour Ordering . . . . . . . . . . . 25

2.3.2 Decoupling Identities . . . . . . . . . . . . . . 32

2.3 .3 Summary . . . . . . . . . . . . . . . . 36

2.4 Spinor Helicity Formalism . . . . . . . . . . . . . . . . 37

2.4.1 Spinor Weight: Little Group Scaling . . . . . . . . . . . 42

2.5 From Feynman Diagrams to On-shell Amplitudes . . . . . . . . . 43

2.6 Tree-Level Amplitudes: Analytic Structure and Factorisation . . . 45

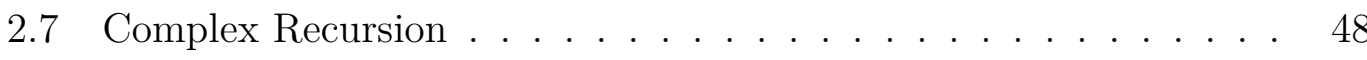

2.7.1 BCFW Recursion . . . . . . . . . . . . . 48

2.8 Loop-Amplitudes . . . . . . . . . . . . . . . . . . . 55

2.8.1 Dimensional Regularisation . . . . . . . . . . . . 56

2.8.2 Integral Reduction of One-Loop Amplitudes . . . . . . . . 57

2.8.3 UV and IR singularities . . . . . . . . . . . . . 59

2.9 Exploiting the Singularity Structure of Loop Amplitudes: Unitarity and Branch Cuts ................ 62 
2.9.1 The Unitarity Method . . . . . . . . . . . . . 64

2.9.2 Generalised Unitarity . . . . . . . . . . . 67

2.9.3 D-dimensional Unitarity . . . . . . . . . . . . . 70

2.9.4 Factorisation of Loop Amplitudes . . . . . . . . . . . 71

2.10 Rational Contributions to Loop Amplitudes . . . . . . . . . . . 73

2.10.1 Complex Recursion Revisited: Augmented Recursion . . . 75

2.10.2 The Rational Part of Two-Loop All-Plus Amplitudes: Double Poles. . . . . . . . . . . . . . . . . 79

2.10.3 Determining the leading current, $\tau_{5 ; 1}^{(1)}\left(\alpha^{-}, \beta^{+}, c^{+}, d^{+}, e^{+}\right) . \quad 84$

2.10.4 Integrating the leading current, $\tau_{5 ; 1}^{(1)}\left(\alpha^{-}, \beta^{+}, c^{+}, d^{+}, e^{+}\right) . \quad .90$

2.10 .5 Calculating Residues . . . . . . . . . . . . 93

3 Analytic all-plus-helicity gluon amplitudes in QCD 97

3.1 Introduction . . . . . . . . . . . . . . . . . 97

3.2 The all-plus helicity amplitude . . . . . . . . . . . . . 100

3.3 Determining $P_{n: 1}^{(2)}$ and Confirming the IR Singularity Structure . . 102

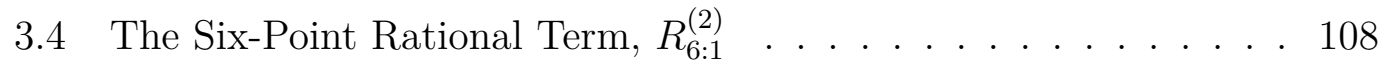

3.5 Factorisations of $R_{6}^{(2)} \ldots \ldots \ldots \ldots \ldots$

3.6 The $s_{a b}$ pole in the Six-Point Rational Term . . . . . . . . . . . 111

$3.7 \quad \lambda_{q}$ Independent $R_{6: 1}^{(2)} \quad \ldots \ldots \ldots \ldots \ldots \ldots$

3.8 The Seven-Point Rational Piece . . . . . . . . . . . . . . . . . 119

3.9 Conclusion . . . . . . . . . . . . . . . . . . . . . 122

4 Colour Dressed Unitarity and Recursion for Yang-Mills TwoLoop All-Plus Amplitudes $\quad \mathbf{1 2 5}$

4.1 Introduction . . . . . . . . . . . . . . . . . 125

4.2 Colour Decomposition of Amplitudes for Colour Dressed Unitarity and Recursion . . . . . . . . . . . . . . . . . . . . . . . . . . . . . . . . . . . . . . . .

4.3 Two-Loop Amplitudes . . . . . . . . . . . . . . . . 135

4.4 Singularity Structure of the Two-Loop All-Plus Amplitudes . . . . 138

4.5 Colour Dressed Unitarity . . . . . . . . . . . . . . . . . . 139

4.6 Recursion . . . . . . . . . . . . . . . . . . . . . . . . . . . . . . . . . . . . . . . . .

4.7 Conclusion . . . . . . . . . . . . . . . . . . . 163 
5 Diagrammar in an Extended Theory of Gravity 165

5.1 Introduction . . . . . . . . . . . . . . . 165

5.2 Determining the Tree-Level $S$-Matrix Elements . . . . . . . . 167

5.3 Soft Limits . . . . . . . . . . . . . . . . . . 176

5.4 Other Theories . . . . . . . . . . . . . . . . 178

5.5 Conclusion . . . . . . . . . . . . . . . . . 179

6 Loop Amplitudes in an Extended Theory of Gravity 181

6.1 Introduction . . . . . . . . . . . . . . . . . 181

6.2 Structure of the Amplitudes . . . . . . . . . . . . . . . . 182

6.3 Yang-Mills Case . . . . . . . . . . . . . . . . . . . . . . . . 184

6.4 Extended Gravity Amplitudes . . . . . . . . . . . . . . . . . . 191

6.5 Beyond Cubic Vertices . . . . . . . . . . . . . . . . . 195

6.6 Conclusion . . . . . . . . . . . . . . . 197

7 Conclusions $\quad 199$

A Feynman Rules for Gluon Amplitudes 203

B Integrals 205

B.1 Integral Functions . . . . . . . . . . . . . 205

B.1.1 Bubble Integrals . . . . . . . . . . . . . . . 205

B.1.2 Triangle Integrals . . . . . . . . . . . . . . . . . . . . . . . . . . . . . . . . . . . . . . . .

B.1.3 Box Integrals . . . . . . . . . . . . . . . 207

C Derivation of the One-Loop Six-Gluon Current 209

C.1 App 2: Integrations . . . . . . . . . . . . . . 218

C.1.1 The $C_{d p}$ piece . . . . . . . . . . . . . . . . 219

C.1.2 The $C_{n f}$ piece................. . . . . 220

D 5-pt all plus - Infra-Red Divergences 227

Bibliography 231 


\section{Acknowledgements}

I'd like to express my deepest gratitude to my supervisor Warren Perkins. His guidance, patience and support encouraged me to improve year by year. Without him I would likely not have had the chance to experience the last four years as part of the Amplitudes group, for that I will always be grateful.

I'd equally like to thank Prof. David Dunbar. His enthusiasm and insight helped me to see the subject from a different perspective.

To Guy Jehu and Joe Strong, working with you both has been a pleasure.

To Roberta, your support (sometimes in cake form) has kept me going. Finally, to my family. Thank you for always keeping me grounded. Grandma, this is for you. 


\section{List of Figures}

1.1 The number of independent Feynman diagrams required to calculate an amplitude increases with the number of external legs [1]. . 3

2.1 Diagrammatic form of the three-point gluon vertex where all momenta are outgoing. . . . . . . . . . . . . . 20

2.2 Diagrammatic form of the four-point gluon vertex where all momenta are outgoing. . . . . . . . . . . . . . 20

2.3 Colour dressed one-mass box diagrams, demonstrating how the trace structures $N_{c} \operatorname{Tr}(a b c d e)$ and $\operatorname{Tr}(a b) \operatorname{Tr}(c d e)$ can arise in a one-loop amplitude. . . . . . . . . . . . . . . 29

2.4 The 'maximally non-factorising' sub-subleading rational contribution to the full colour two-loop five-gluon amplitude, which multiplies the trace structure $N_{c}^{0} \operatorname{Tr}(a b c d e) . \ldots . . . . . . .$.

2.5 Diagrammatic representation of an amplitude factorising as the internal propagator $t_{1 \ldots n}$, goes on-shell. The factorisation includes a sum over internal helicity configurations.

2.6 The origin of the double pole. The double pole corresponds to the coincidence of the singularity arising in the complex threepoint all-plus amplitude and the propagator when the amplitude factorises as $K^{2}=s_{a b} \rightarrow 0 \ldots \ldots \ldots \ldots \ldots$ 


\section{LIST OF FIGURES}

2.7 Diagram containing the leading and sub-leading poles as $s_{a b} \rightarrow 0$. The axial gauge construction permits the off-shell continuation of the internal legs. The two internal helicity configurations must be summed over to obtain the complete contribution to the $1 / s_{a b}$ residue. The one-loop current $\tau_{n}^{(1)}$ can be built from the on-shell $n$-point one-loop single minus amplitude. . . . . . . . . . . 81

2.8 The factorisations that $\tau_{n}^{(1)}$ must reproduce. . . . . . . . . . 83

2.9 When the amplitude is excited with a Risager shift acting on the legs $p_{a}, p_{b}$ and $p_{c}$ with general $\lambda_{q}$, four diagrams corresponding to the rational part of the two-loop all-plus are excited. . . . . . . 94

3.1 Four dimensional cuts of the two-loop all-plus amplitude involving an all-plus one-loop vertex. In the boxes $K_{2}$ may be null but $K_{4}$ must contain at least two external legs. . . . . . . . . . . . . 103

3.2 The non-vanishing quadruple cut. $A$ is a MHV tree amplitude whereas $B$ is a one-loop all-plus amplitude. The set $K_{2}$ may consist of a single leg but the set $K_{4}$ must contain at least two legs. The integral function depends upon $S \equiv\left(p_{i-1}+K_{2}\right)^{2}$ and $T \equiv\left(K_{2}+\right.$

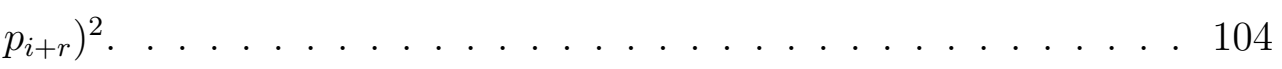

3.3 The one-loop to one-loop factorisation that corresponds to the factorisations derived in the current, $C_{d p}$ and $C_{s b}$. $C_{d p}$ corresponds to the factorisation involving the one-loop three-point all-plus and the one-loop five-point single-minus.

4.1 The origin of the double pole. The double pole arises due to the coincidence of the singularity arising in the one-loop three-point all-plus vertex, and the propagator defining the channel in the factorisation corresponding to $K^{2}=s_{a b} \rightarrow 0$. The helicities on the right hand side of the factorisation are general. . . . . . . . 132

4.2 Four dimensional cuts of the two-loop all-plus amplitude involving an all-plus one-loop vertex. For the five-gluon amplitude, we take $K_{2} \rightarrow 0$, and as always $K_{4}^{2} \neq 0 \ldots \ldots \ldots \ldots$ 


\section{LIST OF FIGURES}

4.3 Four-dimensional quadruple cuts uniquely define the coefficients of the box integral functions. Extended to a full colour amplitude, the cut diagrams appear as three types of boxes reflecting the three types of colour structures contained in the full colour four-point all-plus one-loop amplitude. . . . . . . . . . . . . . . 144

4.4 The box diagram considered in the quadruple cut with explicit colour labels and internal helicity configuration. . . . . . . . . 145

4.5 Diagram containing the leading and sub-leading poles as $s_{a b} \rightarrow 0$. The axial gauge construction permits the off-shell continuation of the internal legs. . . . . . . . . . . . . . . . . . 154

4.6 The rational piece, $R_{5: 3}^{(2)}$, multiplying the trace structure $N_{c} \operatorname{Tr}[a b] \operatorname{Tr}[c d e]$, is constructed from currents of the form $\tau_{5: 1}^{(1)}\left(\alpha^{ \pm}, c^{+} ; \beta^{\mp}, d^{+}, e^{+}\right) . \quad$. 158

4.7 The maximally non-factorising rational piece, $R_{5: 1 B}^{(2)}$, multiplying the trace structure $\operatorname{Tr}[a b c d e]$, is constructed from currents of the form $\tau_{5: 3}^{(1)}\left(\alpha^{ \pm}, e^{+} ; \beta^{\mp}, c^{+}, d^{+}\right) \ldots \ldots \ldots \ldots \ldots$

5.1 The non-zero three-point amplitudes. . . . . . . . . . . . . . . . 169

5.2 General factorisation for an $n$-point $\mathcal{O}(\alpha)$ all-plus amplitude. The $\mathcal{O}(\alpha)$ amplitude can only enter the factorisation on the side of the on-shell propagator with a positive helicity. . . . . . . . . 170

5.3 The only type of factorisation of the four-point $\mathcal{O}(\alpha)$ single-minus. 172

5.4 Factorisations of the five-point single-minus amplitude. . . . . . . 174

5.5 Factorisations of the five-point MHV amplitude . . . . . . . . . . 175

5.6 Factorisations of the four-point MHV amplitude at $\alpha^{2}$. . . . . 179

6.1 The two-particle cut of the all-plus amplitude. . . . . . . . . . 187

6.2 The bubbles for single minus . . . . . . . . . . . . . . . . 189

6.3 Factorisations of the four-point MHV amplitude at $\alpha^{2}$. . . . 196 


\section{Chapter 1}

\section{Introduction and Historical Overview}

The field of amplitudes is one for which theory meets experiment. When thinking about the way physics was conducted traditionally, one imagines taking some physical theory and performing an experiment to test the observables. For high energy particle physics, the data we need to gather in order to test our theories is obtained from colliders experiments. For example, collider experiments performed at the Large Hadron Collider (LHC) are vital to a full understanding of the Standard Model of particle physics and possible deviations from it. In the LHC, high energy beams of protons are fired at one another producing swarms of particles, whose signatures are measured at detectors on the boundary. The signatures are quantified and measured using the total cross-section of all possible particle interactions that can occur between two protons in a beam. This is an object that gives a probability of all the various interactions that can occur. At high energies, the parton model [2, 3] provides a basis for analysing total crosssections. The parton model is an illustration of the hadrons that comprise particle collisions through quantum chromodynamics (QCD). Its key feature being that the hadrons contain a multitude of subatomic particles and gauge boson - partons. In the case of the proton 1 , deep inelastic scattering experiments [4, 5] uncovered that three quarks are confined inside the proton by way of gluon interactions.

\footnotetext{
${ }^{1}$ at low energies
} 


\section{INTRODUCTION AND HISTORICAL OVERVIEW}

At higher energies, the proton can contain many particle anti-particle pairs and many more gluons. The parton model and the QCD factorisation theorem tell us that the total cross-section between two protons is written as the convolution of parton distribution functions (PDF's) and a partonic cross-section. The PDF's are a non-perturbative quantity that describe the composition of the hadrons involved in the scattering, these are determined experimentally. While the partonic cross-section is a perturbative quantity that describes the hard scattering interactions between the partons.

The majority of the signal detected in hadron-hadron collisions comes from jet signals that are considered as 'noise' in the detector since they can obscure more interesting signals. A large portion of these jets originate from gluon interactions. If we are to test the predictive strength of the Standard Model, understand deviations from it, or accurately look for resonances belonging to new unidentified particles, these jet signals must be understood to higher and higher degrees of precision. This amounts to understanding, in part, gluon amplitudes order by order in perturbation theory that contribute to the partonic cross sections.

Since we can model the total cross-section from a theoretical standpoint and measure such a quantity in a collider experiment, we are provided with a testable link between theory and experiment.

We approach this collaboration from the theoretical perspective. In this thesis, we consider a theory of pure Yang-Mills [6]. We consider only gluon interactions and promote the gauge group from $S U(3)$ to, in general, $U\left(N_{c}\right)$. Protons involved in collisions at the LHC are ultra-relativistic. In this regime the coupling constant of QCD is small, and we can take a pertubative approach to calculating the amplitudes that describe gluon interactions in an expansion of the coupling constant.

Traditionally, amplitudes are computed by deriving Feynman rules from the Lagrangian of the theory in question and calculating every Feynman diagram [7] that contributes to the scattering process of interest. For low multiplicities and at leading order in perturbation theory, this method can be used successfully with a fair amount of effort. However, we are concerned with more complicated interactions i.e. higher multiplicity and higher order amplitudes. This poses a 


\begin{tabular}{|l|l|l|l|l|l|l|l|}
\hline $\mathrm{n}=$ & 4 & 5 & 6 & 7 & 8 & 9 & 10 \\
\hline- & 4 & 25 & 220 & 2485 & 34300 & 559,405 & $10,525,900$ \\
\hline
\end{tabular}

Figure 1.1: The number of independent Feynman diagrams required to calculate an amplitude increases with the number of external legs [1].

problem for the traditionalist, since they will have to calculate hundreds to thousands of diagrams, with each diagram being comprised of unwieldy expressions. Fig. 1.1 illustrates how many Feynman diagrams contribute to a calculation at tree-level for an $n$-pt gluon scattering amplitude. For comparison at one-loop, the seven-point gluon amplitude contains 227,585 diagrams.

Moreover, each Feynman diagram is a gauge dependent object, with gauge independence only restored in the full sum of Feynman diagrams contributing to an amplitude. The lack of an appropriate way to group these Feynman diagrams together into gauge invariant subsets meant that there is a vast amount of redundancy. This redundancy manifests itself as large expressions for an amplitude that are subject to a multitude of cancellations and simplifications resulting in compact formulae. These sorts of scenarios often to point to a misunderstanding, the notion that there must be a better way to formulate the problem.

A breakthrough emerged in the late 1980's with the calculation of the treelevel six-gluon amplitudes in Yang-Mills [8, 9]. The authors utilised a new technique in calculating multi-gluon scattering, inspired by Chan-Paton factors [10] arising in string theory. It was shown that the colour information could be used to organise the amplitude as 'colour $\times$ kinematics', where the kinematics were labelled as the 'subamplitudes'. These subamplitudes - referred to as partial amplitudes in this thesis - are gauge invariant objects with a symmetry governed by the colour trace structure they multiply. This trace based colour decomposition is achieved by rewriting the structure constants, that carry the colour information of the vertices, as traces of the group generators of the external gluons in the fundamental representation. The full tree-level amplitude could then be expressed as a sum over all the independent diagrams multiplied by their colour trace structures, given by a sum over non-cyclic permutations of a tree-level amplitude. Additionally, the partial amplitudes present themselves with symmetries 


\section{INTRODUCTION AND HISTORICAL OVERVIEW}

that aid in the calculation of the full tree-level amplitude. They can be related to each other via cyclic shuffling of the legs, a flip symmetry and decoupling identities, then referred to as dual Ward identities [8, 11]. Colour decomposition is now a well established procedure in the literature and can at times be taken for granted, but it has great significance in the genesis of the modern approach - the amplitude could be organised into gauge invariant subsets eliminating some redundancy from the outset. With this insight, researchers could focus on calculating the gauge invariant partial amplitudes and reduce the scale of the computation. Other colour decompositions have emerged since [12 14] that are useful for calculating cross-sections or for efficiency improvements in numerical evaluation. Indeed, comparison between the various colour decompositions has lead to more complete relations between partial amplitudes at different orders of colour $[15,16]$.

Following the expression for a six-gluon amplitude in 1985 [17], an important understanding of the simplicity of the partial amplitudes arose with the conjecture for the $n$-gluon MHV amplitude ${ }^{1}$ by Parke and Taylor [19]. However, this conjecture was written in terms of four momenta. Through the work of the CALKUL collaboration [20 25] and an extension to non-abelian gauge theories by [26] it was found that polarisation vectors could be expressed in the helicity basis and the most compact way to express gluon amplitudes was through the spinor helicity formalism. This method allowed on-shell momenta to be expressed using two-component Weyl spinors and lead to compact six-point expressions [8, 9].

Tree-level calculations made a leap forward when Witten [27] found a dual definition of Yang-Mills using Penrose's twistor space. Fourier transforming from the usual description of momentum space scattering amplitudes to Pensrose's twistor space [28], allowed Witten to show that non-vanishing amplitudes in Yang-Mills live on specific curves in twistor space. In addition, Witten motivated an alternative way of viewing the three-point amplitudes of the theory. In particular for amplitudes with all but one gluon having positive helicity, the amplitudes are described by a curve of degree zero - a point. This implies that $p_{i} \cdot p_{j}=0$, for all $i, j \in n$, which is only valid for $n=3$. The three-point MHV amplitude

\footnotetext{
${ }^{1}$ The conjecture was later proven by Berends and Giele [18] using off-shell recurrence relations, whereby an $n$-point amplitude is found using an $(n-1)$-point off-shell current.
} 
vanishes for real momenta in Minkowski space with the signature (+- - ), but is well-defined and can be written in a Parke-Taylor like expression in a different signature, or by using complex momenta. This prompted a new method for calculating tree-level amplitudes in Yang-Mills using MHV amplitudes appropriately continued off shell as vertices, known as the CSW formalism [29].

By this point Britto, Cachazo and Feng developed generalised unitarity in the pursuit of calculating one-loop amplitudes in $\mathcal{N}=4 \mathrm{SYM}$ [30]. At one-loop this supersymmetric theory can be expressed as a sum over six types of scalar box integrals, massless through to four-mass boxes. Quadruple cuts of these boxes uniquely determine the integral functions coefficients, hence the amplitude is determined by reading off the cut expression. The IR singular terms of all $n$-point one-loop QCD amplitudes are well understood [31-33] and are proportional to the same helicity $n$-point tree-amplitude. Since $\mathcal{N}=4$ SYM is simpler than QCD, in that its integral basis only contains box integral functions - the IR singular terms in $\mathcal{N}=4 \mathrm{SYM}$ are determined by the sum of the box integral functions multiplied by coefficients. Thus the box coefficients are related to the $n$-point treelevel amplitude. This lead to a definition of tree-level amplitudes as a sum of the coefficients of the various box integrals appearing in one-loop amplitudes in $\mathcal{N}=4$ SYM [34]. The culmination of this line of work lead to the now famous tree-level recurrence relation [35] based on factorisation theorems for tree-level amplitudes. Developing a complex shift that altered the position of the spinors of two gluons in complex momentum space allowed the amplitude to become a meromorphic function in the complex shift parameter $z$. In conjunction with the existence of the complex valued three-point MHV and Googly amplitudes [27], the authors showed that a tree-level amplitude can be calculated by considering complex analysis and factorisations of a tree-level amplitude as various $z$-dependent propagators go onshell [36].

Understanding that an amplitude in Yang-Mills could be calculated recursively via its pole structure and factorisation theorems caused a surge of developments in the field of amplitudes at tree-level and for a variety of one-loop amplitudes (both pure gluon and more phenomenologically related amplitudes ${ }^{2}$ ) [37-

\footnotetext{
${ }^{1}$ dubbed the BCFW shift

${ }^{2}$ The list of references is a small sample of the work, but shows the scope of the method.
} 


\section{INTRODUCTION AND HISTORICAL OVERVIEW}

43]. Since the BCFW relations rely on factorisation theorems where an internal propagator goes on-shell, it had a natural extension to other physical theories. Indeed this was found to be the case for tree-level gravity amplitudes [44]. Benincasa and Cachazo [45], introduced a set of consistency conditions of the $S$ Matrix based on factorisation of tree-level amplitudes. Theories that admitted construction from three-point vertices with a non-trivial $S$-matrix were deemed 'constructible', and 'fully constructible' if this approach extended to determining all n-point amplitudes.

Interest in amplitudes calculations moved on to one-loop calculations before fully understanding tree-level amplitudes. The necessity of investigating one-loop amplitudes arose from the need for more precise predictions for theoretical crosssection calculations to compare with experiment. Without the next-to-leading order calculations, we can only predict the magnitude of the cross-section without any added precision.

The S-Matrix program of the 1960's [46] attempted to determine amplitudes via their singularity structure and the unitarity condition of the $S$-matrix. The unitarity condition, in essence, expressed the discontinuity over a branch cut contained in an $L$-loop amplitude as the product of $(L-1)$-loop amplitudes using Cutkosky's cutting rules [47]. However, at one-loop this amounted to calculating dispersion integrals that described the branch cut singularity structure of a oneloop amplitude. Lacking sufficient additional constraints, this method produced generalised dispersion relations, which are far too computationally involved to be of practical use. Progress in this vein met a dead end until the theory of QCD emerged, allowing an amplitude to be expressed as a sum of Feynman integrals. The integrals range over all momenta that the unconstrained loop momenta can take, i.e. $\infty \leq \ell \leq+\infty$ and are plagued by divergences at high (ultraviolet) and low (infrared) energies in $D=4$ dimensions. This was rectified by analytically continuing to $D=4-2 \epsilon$ dimensions with dimensional regularisation [48], shifting the divergences to poles in $\epsilon$ as $\epsilon \rightarrow 0$. With the added perspective of QCD, it was shown that a general one-loop $n$-point tensor integral could be reduced to a sum of box, triangle and bubble integral functions [49 51] with rational coefficients, 
through Passarino-Veltman reduction [52]. Applying the reduction in four dimensions amounts to rational ambiguities at $\mathcal{O}\left(\epsilon^{0}\right)[53]$ that must be determined via a different route. Alternative methods emerged for dimensionally regulated amplitudes, keeping track of the $-2 \epsilon$ dimensional degrees of freedom [54, 55]. The integral basis thus restricts the space of transcendental functions (and thus branch cut structures) that can arise at one-loop. Leaning on this description of the amplitude, the authors of refs. [53, 56] realised by considering Cutkosky's rules in four dimensions 1 and promoting the cut integrals to covariant integrals, dispersion relations could be circumvented. The unitarity method matches integrals arising from the cuts to integrals in the basis, identifying the coefficients. The unitarity method substantially simplified the approach to one-loop amplitude calculations and lead to a more manageable technique.

Double cuts of an amplitude were the primary incarnation of this method. In the modern approach, we are comfortable with more complicated cuts - cutting more than two propagators at a time to isolate more specific branch cut structures described by particular integral functions. This idea has its roots in the work of the $S$-Matrix program [46] but triple cuts were employed to calculate one-loop amplitudes [57] and the splitting functions of two-loop amplitudes [58]. However, its now accustomed form came in the calculation of one-loop $N M H V$ and $N^{2} M H V$ amplitudes in $\mathcal{N}=4 \mathrm{SYM}$ [30]. The authors found that applying a maximal cut, i.e. cutting all four propagators of a one-loop box integral, constrained the loop momenta entirely. This demonstrated that the box integral coefficient could be given by a product of four tree-level amplitudes summed over the solutions given by the cut constraints. Although ref. [30] approached the issue of a vanishing real valued three-gluon amplitude in the $(+----)$ signature by using an alternative metric, inspiration from Witten [27], allowed for an easier path by using complex momenta in the $(+----)$ signature. This method had two impacts on the community; the aforementioned major breakthrough in tree-level calculations [36] and it also led to a variation in the canonical unitarity method in the form of generalised unitarity. Systematically applying quadruple

\footnotetext{
${ }^{1}$ applied in all channels
} 


\section{INTRODUCTION AND HISTORICAL OVERVIEW}

cuts to fix the box coefficients, then applying triple cuts and double cuts, allows the extraction of all integral coefficients in a hierarchical fashion designed to decrease the complexity of the calculation. Lower order cuts that don't fix the loop momenta entirely, like a triple or double cuts, have degrees of freedom that remain in the cut to be integrated over. Methods based on complex analysis [59] and evaluating a basis of possible integrals that can arise from the cuts [60], emerged to calculate the coefficients from triple and double cuts.

The philosophy above relates to the four-dimensional unitarity method that relies on performing cuts in four-dimensions, disregarding the $-2 \epsilon$ dimensional part of the loop momenta. Another method that is widely used is $D$-dimensional unitarity 61 66], that keeps track of these dimensionally regulated degrees of freedom. This method requires a $D$-dimensional reduction of an $n$-point loop amplitude [67, 68]. In this framework, the cut constructible pieces can be calculated in a similar manner to the above case, however the rational terms are identified as arising from higher dimensional integrals depending on the $-2 \epsilon$ dimensional components of the loop momenta [62, 63, 69, 70]. All rational contributions to one-loop amplitudes have been identified from the integral reduction [71].

Not all loop-amplitudes contain transcendental functions that have branch cuts in the kinematic invariants. The all-plus and single minus amplitudes are rational at one-loop, owing to the fact their tree-level counterparts vanish 72 74]. These rational functions only contain collinear and multiparticle poles, in fact the highest order pole for these one-loop amplitudes in QCD are second order poles, arising from a factorisation of the amplitude where one of the factorised amplitude is the complex one-loop three-point all-plus amplitude. In theory, these amplitudes are obtainable from the tree-level recursive methods outlined by refs. [35, 36], general factorisation theorems of one-loop amplitudes [38], collinear limits of one-loop amplitudes [53] and soft theorems [75, 76]. The one-loop allplus amplitude was conjectured by Bern, Chalmers, Dixon and Kosower [77, 78. through string theory methods and also by a collinear bootstrap method $[56$, 69, 77, 78] and was later proven by Mahlon [79]. The one-loop single-minus $n$ point amplitude was written down by Mahlon [79] using a generalisation [80] 
of the Berends-Giele recurrence relations [18]. The single-minus and all-plus amplitudes were interestingly revisited in ref. [38] using BCFW recursion. This amplitude contains double poles that alter the form of the recurrence relations used to derive the $n$-point single minus amplitudes. Examining the five-point single minus amplitude as a guide to a general expression, the authors found that three of the four terms in the expression could be generated via the factorisation theorem given by BCF [35]. However, the subleading single pole arising from the double pole cannot be found this way. Instead, the subleading contribution from the double pole term was built from a combination of soft factors.

This is a very important point, rational parts of loop amplitudes can contain double poles in the spinor variables, whose Laurent series contains a subleading single pole contribution that general factorisation theorems are insensitive to.

Augmented recursion, is a method developed to determine coefficients of subleading poles in a Laurent series about double pole contributions (or in principle, higher order poles) to rational amplitudes, or rational parts of loop amplitudes. In particular, for an amplitude that contains simple and double poles; factorisation theorems and BCFW recursion can be used to determine the coefficients of simple pole contributions and leading singularities of higher order poles - augmented recursion is required to determine the remaining subleading poles. It has been applied to a variety of one-loop leading in colour Yang-Mills and gravity amplitudes [81 84] and also the leading in colour two-loop all-plus Yang-Mills amplitudes [85 88] with a generalisation to the full colour rational parts of the fiveand six-gluon amplitudes in Yang-Mills [89 91]. The methods found in [89, 91] are simply extended to seven-point and are currently under way. This method is examined in exhaustive detail in later chapters, however the principle is very similar to both [38, 79], in that one constructs a doubly off-shell current and in doing so exposes the subleading pole structure belonging to the Laurent series of the double pole. This is combined with the knowledge that at one- and two-loops the double poles arise from factorisations of the amplitude that involve the one-loop all-plus three-point vertex that is singular when the two external legs go collinear. As such the doubly off-shell current that is derived from the $(L-1)$-loop, $n$-point single-minus (and MHV) amplitude for the $L$-loop all-plus 


\section{INTRODUCTION AND HISTORICAL OVERVIEW}

(single-minus) amplitude must reproduce these factorisations up to additional non-factorising contributions.

Two-loop amplitudes represent the state of the art for the field of amplitudes in non-supersymmetric pure Yang-Mills. The full colour all-plus four-point gluon helicity amplitude in 2000 [70] was the first two-loop amplitude computed using a unitarity based approach. It was swiftly succeeded by a description of all helicity amplitudes at two-loop four-point in QCD [92] that found agreement with ref. [93] which calculated the tree-level to two-loop interference for cross section calculations. Many issues make life harder at two-loops; the lack of a general basis of integral functions and more complicated Feynman integrals pose a significant obstacle to practical calculation. Beyond four-point, the authors of ref. [94] calculated numerically, the leading in colour five-point gluon amplitude [94] using a generalised OPP reduction [67 and $D$-dimensional unitarity. The full colour numerical result followed shortly after [95]. Analytic results at five-point came with the all-plus [96], again using $D$-dimensional unitarity and a technique for relating all the integrals present to a set of ad hoc master integrals, via differential equations [97]. Gehrmann et al. showed that the two-loop all-plus could be reorganised in terms of its singularity structure in the dimensional regulator $\epsilon$. The amplitude is composed of IR divergent terms comprised of soft divergences, finite polylogarithmic parts and a rational piece.

These $D$-dimensional unitarity techniques have led to many advancements at two-loop five-point, including full colour results for five-point amplitudes and leading in colour single-minus and MHV amplitudes [98 110]. Despite its obvious success, $D$-dimensional unitarity techniques have been thus far unable to reach higher multiplicities at the all-plus level. Four-dimensional methods uncover the fact that the one-loop integral basis may be applied to this specific two-loop amplitude, as cuts of the one-loop all-plus amplitude vanish. The one-loop all-plus amplitude appears as a tree-like rational vertex in the corner of one-loop box, triangle and bubble diagrams that contribute to the two-loop amplitude. This major simplification allowed a combination of four-dimensional unitarity and augmented recursion to be applied to the helicity amplitude, leading to compact forms of the five-, six- and seven-gluon leading in colour amplitudes [86 88]. Since the cut 
constructible parts of the amplitude are easily calculated using one-loop methods, a general $n$-gluon expression was given for the IR and polylogarithmic parts of the all-plus [111], whose IR terms agreed with the predicted forms given by Catani [33]. The bottleneck to an all- $n$ form of the full amplitude comes from the derivation of the rational piece via augmented recursion. However, the general method applied at leading in colour has recently been extended to full colour results via colour dressed unitarity and recursion up to six-point [89, 91]. Additionally, the first sub-subleading partial amplitude in the colour decomposition has been given to all- $n$ [90]. The four dimensional helicity method and augmented recursion are the computational schemes we review and use in this thesis.

The structure of the thesis is as follows. In chapter 2 we outline all the technology we require to describe the papers that form the foundations of chapter 3 [88], chapter 4 [89], chapter 5 [112] and chapter 6 [113]. We introduce the two theories of interest in this thesis, pure Yang-Mills and pertubative gravity. We then outline the colour decomposition and the spinor helicity formalism that organise the amplitudes into compact gauge invariant partial amplitudes. Then, the derivation of low multiplicity tree-level amplitudes are discussed, from the Feynman diagram perspective and we constrain the form of the three-point amplitudes from Lorentz invariance and locality. Subsequently, we develop on these three-point complex trees to build higher multiplicity tree-level amplitudes using factorisation theorems and BCFW recursion. Later, loop amplitudes are introduced and we show how we dimensionally regulate amplitudes and show some universal divergent properties of unrenormalised Yang-Mills amplitudes. We then discuss how a one-loop amplitude is reduced to a basis of scalar integrals and demonstrate the unitarity and generalised unitarity methods. After outlining the important factorisation properties of one-loop amplitudes, we thoroughly demonstrate augmented recursion with an explicit example ascertaining the double pole contribution to the rational part of the two-loop five-gluon all-plus amplitude. Since this technique is used in both chapter 3 and chapter 4 , we dedicate a large portion of the thesis to adequately explain the method.

Chapter 3, is based on the work performed in ref. [88]. In this chapter we determine the leading in colour two-loop seven-gluon all-plus amplitude in Yang- 


\section{INTRODUCTION AND HISTORICAL OVERVIEW}

Mills. The IR divergent parts of the amplitude have been given in general by Catani [33], and the $n$-point finite polylogarithmic parts were worked out in [111]. The major contribution to the field comes from deriving the rational part of the amplitude. Despite this, we take the time to review the $n$-point calculation from a four-dimensional unitarity perspective before moving on to determining the rational part of the amplitude. The improved techniques developed in this derivation of the seven-point rational piece 1 were developed in conjunction with an improvement for the six-point rational piece. As the six-point rational piece is more manageable to deal with, we show this improvement in terms of the sixpoint rational term and present the seven-point rational piece at the end of the chapter.

Chapter 4 extends the work on four-dimensional unitarity and augmented recursion from its application at leading in colour, to determining a full colour amplitude - the two-loop five-point all-plus. Casting the amplitude in terms of $U\left(N_{c}\right)$ colour factors we directly determine both the physical and non-physical partial amplitudes by colour dressing the cut integrals and augmented recursion calculation with full colour amplitudes instead of the leading in colour partial amplitudes. The advantage of working in $U\left(N_{c}\right)$ Yang-Mills comes from additional consistency checks afforded to us from the decoupling identities that relate some of the partial amplitudes. In this context they act as independent checks on the directly evaluated partial amplitudes. The sub-subleading single trace partial amplitude, $A_{5: 1 B}^{(2)}$, is the only partial amplitude that is not related to another partial amplitude via decoupling relations, and thus is not uniquely specified by its decoupling identity. To resolve this, we use additional five-point specific group theory relations [15, 16] that specify this amplitude in terms of other partial amplitudes. We verify the full colour IR divergences predicted by Catani $[33]^{2}$ and the results obtained in ref. [110]. We present the full colour forms for the finite polylogarithmic functions arising from the full colour box integrals and show a major development in augmented recursion. We demonstrate that only the leading in colour one-loop current ${ }^{3}$ is required to calculate all subleading in colour

\footnotetext{
${ }^{1}$ compared with the original implementations at two-loop for the five- and six-point rational pieces, originally calculated in [87]

${ }^{2}$ and present another form of the all- $n$ IR divergences of this amplitude in Appendix D.

${ }^{3}$ which in essence defines the most complicated portion of the rational piece
} 
rational terms.

Chapter 5 deals with determining the tree-level $S$-Matrix elements of a theory of gravity, extended by the term $\alpha R^{3}$. This theory defines the counterterms for two-loop Einstein-Hilbert gravity. As a foundation, we use the tree-level three-graviton amplitudes from Einstein-Hilbert (EH) gravity, and we constrain the forms of the three-point amplitudes given by the extension at $\mathcal{O}(\alpha)$ using Lorentz invariance and locality. From these grounds we recursively construct the higher multiplicity graviton amplitudes at $\mathcal{O}(\alpha)$ using $\mathrm{BCFW}$ recursion, involving a tree-level EH amplitude and an $\mathcal{O}(\alpha)$ tree-level amplitude given by the extension. Collinear limits are employed as checks on these amplitudes, in addition to soft limits. Graviton amplitudes satisfy universal [114 116$]$ soft limits to sub-subleading order [115, 117] and we verify this is the case for each helicity amplitude in the extended theory of gravity up to $n=8$. We also briefly consider this approach to other extensions, both the at a higher order in $\alpha$ and by the extension of an additional $R^{4}$ term.

Chapter 6 considers the same theory at the next order in perturbation theory. Using the integral basis of one-loop amplitudes and four-dimensional unitarity techniques we examine the renormalisability of such a theory by considering the UV divergences arising from the bubble integral functions. As a comparison to this approach, we also consider the extension of pure Yang-Mills by an $\mathcal{O}(\alpha) F^{3}$ terms. For extended Yang-Mills, we find that the UV singularity at $\mathcal{O}(\alpha)$ is proportional to the $\mathcal{O}(\alpha)$ tree-level amplitude and a redefinition of the coupling constant provides an appropriate counterterm to cancel the divergence. In the extended gravity case however, the UV singularities are not proportional to the tree amplitude and higher dimensional operators - corresponding to an additional four point vertex in the diagrammar approach - must be added to renormalise the theory. 
1. INTRODUCTION AND HISTORICAL OVERVIEW 


\section{Chapter 2}

\section{Review of Techniques for Calculating Scattering Amplitudes}

Amplitudes are an important bridge between theories of particle physics and experiment. They are essential components of the observables constructed from a Quantum Field Theory and thus allow us to test the legitimacy and accuracy of a particular theory.

In this chapter we briefly discuss the role that amplitudes play in comparison to experimental measurements, through cross-sections. In section 2.1 we introduce the theories that describe the interactions of the amplitudes we are interested in calculating, namely pure Yang-Mills and Gravity, and we present the perturbative approach to calculating amplitudes in these theories in section 2.2. The link from the Lagrangian approach to an amplitude calculation, is the derivation of the Feynman rules that govern the allowed interaction vertices of a theory. In Yang-Mills, these are a product of colour factors and kinematics. Generating an amplitude using these Feynman rules leads to often unwieldy intermediate expressions before large cancellations occur. Much work has been performed in reducing this calculational complexity, in the form of a reorganisation of the colour factors and a more compact representation for the momenta of the interacting particles. Colour ordering and the spinor helicity method are presented in section 2.3 and section 2.4 respectively. 


\section{REVIEW OF TECHNIQUES FOR CALCULATING SCATTERING AMPLITUDES}

In section 2.5 we determine the complex three-gluon amplitudes in Yang-Mills in two fashions and find agreement. First, by considering the Feynman rules for gluon interactions, and secondly by fixing the form of the three-gluon amplitudes by Lorentz invariance and locality. The second of which is the basis for calculating rational amplitudes with single poles, in particular tree-level amplitudes through the recursive BCFW formalism. This is outlined in section 2.7.

Loop amplitudes that contribute to higher orders in perturbation theory are introduced and discussed in section 2.8. The unconstrained loop momenta are integrated over, and in four dimensions these integrals are divergent in the high energy (ultraviolet) and low energy (infrared) regions of the loop integration. We tame these infinities through dimensional regularisation, and present the UV and IR divergences of one-loop (and a special two-loop case: the all-plus) amplitudes after reducing the problem to a set of integral basis functions. In general, these loop momenta contain branch cut singularity structures plus additional rational terms. We discuss the unitarity method, which utilises the branch cut singularity structures and the integral basis for a general one-loop amplitude, to calculate the 'cut constructible' parts of the amplitude in section 2.9.

This chapter concludes with section 2.10, where we discuss the rational parts of one-loop amplitudes and the two-loop all-plus amplitude. These amplitudes can have double poles that obstruct the general implementation of the BCFW technique. As such we have developed a technique to determine the rational terms despite this - augmented recursion. We take the time to give a thorough calculation of the necessary elements to determining the rational pieces in this fashion.

Returning to the link between theory and experiment, consider the total crosssection given by the collision of two partons with momentum $x_{1} p_{1}$ and $x_{2} p_{2}$, in a collider experiment. At high energies the QCD Factorisation Theorem tells us that a total cross-section is a convolution of the parton distribution functions (PDF's) $f_{i}$ and $f_{j}$ of the colliding partons and the partonic cross-section, $\hat{\sigma}_{i j}$ 
related to the hard-scattering process [118],

$$
\sigma\left(p_{1}, p_{2}\right)=\sum_{i, j} \int d x_{1} d x_{2} f_{i}\left(x_{1}, \mu^{2}\right) f_{j}\left(x_{2}, \mu^{2}\right) \hat{\sigma}_{i j}\left(x_{1} p_{1}, x_{2} p_{2}, \alpha\left(\mu^{2}\right), \frac{Q^{2}}{\mu^{2}}\right)
$$

The PDF's are non-perturbative objects that depend on the Bjorken variable $x$, the coupling constant $\alpha$, the factorisation scale $\mu$ and the energy scale of the hard scattering $Q$. They describe the long range behaviour of the partons and are a probability density of finding a quark or gluon inside the hadron in question, namely a proton in the case of LHC experiments. The DGLAP evolution equations [119 121] show the variation of the PDF's with $Q$. It facilitates the evolution of the PDF's between different energy scales, such that measurements of the functions at particular collision energy can be used for collisions at another energy. Although QCD predicts the scaling behaviour with respect to $Q$, it cannot predict the form of the PDF's with respect to $x$ - the PDF's must be determined experimentally.

On the other hand, the partonic cross-section describes the short range interactions. Since it depends on the coupling constant, at high energiest it is a perturbative object, that is comprised of all the hard-scattering processes that can contribute to interactions between the partons [118],

$$
\hat{\sigma}_{i j}=\sum_{k} c_{i j, k} \alpha^{k}
$$

The coefficients of the coupling constant are the scattering cross-sections that contribute to a hard scattering event at each order in perturbation theory. They are proportional to the square of an amplitude summed over colours. In general they would be summed over all contributing particles, however we will only consider the purely gluonic contributions. In the next section, we define the theories responsible for the amplitudes we will calculate.

\footnotetext{
${ }^{1}$ where $\alpha<<1$
} 


\section{REVIEW OF TECHNIQUES FOR CALCULATING SCATTERING AMPLITUDES}

\subsection{Pure Yang-Mills and Pertubative Gravity}

In this section, we introduce the two theories of interest in this thesis that describe the amplitudes; pure Yang-Mills and pertubative gravity. We briefly discuss the definition of these theories with enough information to move on to the modern approach using on-shell gauge invariant partial amplitudes.

\subsubsection{Pure Yang-Mills}

The Lagrangian for pure Yang-Mills takes the form:

$$
\mathcal{L}=-\frac{1}{4} \operatorname{Tr}\left(F_{\mu \nu} F^{\mu \nu}\right)
$$

where the field strength tensor $F_{\mu \nu}$ is composed of derivatives of the gluon-field $A_{\mu}$

$$
F_{\mu \nu}=\partial_{\mu} A_{\nu}-\partial_{\nu} A_{\mu}+i g f^{a b c} A_{\mu}^{b} A_{\nu}^{c}
$$

Imposing that the theory is invariant under local transformations amounts to the fact that the gluon fields, $A_{\mu}$, are invariant under the gauge transformations described by $S U\left(N_{c}\right)$. We can extend $S U\left(N_{c}\right)$ to $U\left(N_{c}\right)$, since $U\left(N_{c}\right)=S U\left(N_{c}\right) \times$ $U(1)$ and we consider both gauge groups in this thesis. The transformations can be described by the generators of the group via

$$
U(x)=e^{i T^{a} x^{a}}
$$

where the $T^{a}$ are the traceless Hermitian generators that span the space of the transformation and $x^{a}$ is a continuous variable.

The structure constants $f^{a b c}$ are defined by the commutator of the gauge boson generators in the fundamental representation,

$$
\left[T^{a}, T^{b}\right]=i f^{a b c} T^{c}
$$


that are normalised according to

$$
\operatorname{Tr}\left[T^{a} T^{b}\right]=\delta^{a b}
$$

Gauge fixing the Lagrangian using the axial gauge term,

$$
L_{g f}=-\frac{1}{\xi}\left(q^{a} A_{a}^{\mu}\right)^{2},
$$

imposes that the ghosts decouple from the gluons, meaning that we can ignore them. The gluon propagator is then given as

$$
\frac{i}{p^{2}} d_{\mu \nu}=\frac{i}{p^{2}}\left(-\eta_{\mu \nu}+2 \frac{p_{\mu} q_{\nu}+q_{\mu} p_{\nu}}{2 q \cdot p}\right)
$$

where the metric is the Minkowski metric in the Lorentz signature (+ - - -) and we have selected the lightcone gauge, which ultimately amounts to setting $\xi=0$ and $q^{2}=0$ once the gluon propagator has been derived 1 . The lightcone gauge is the most natural gauge to be used when working with the spinor helicity formalism. The axial gauges only permit two physical polarisation states of the gluons to exist which satisfy

$$
\begin{aligned}
& \epsilon_{\mu}(p, q) \cdot p^{\mu}=0, \\
& \epsilon_{\mu}(p, q) \cdot q^{\mu}=0 .
\end{aligned}
$$

Extracting the Feynman rules in the canonical way leads to the following allowed three-gluon and four-gluon interactions,

$$
V_{3}\left(p_{1}^{\mu}, p_{2}^{\nu}, p_{3}^{\rho}\right)=i g f^{a b c}\left[\eta^{\mu \nu}\left(p_{1}-p_{2}\right)^{\rho}+\eta^{\nu \rho}\left(p_{2}-p_{3}\right)^{\mu}+\eta^{\rho \mu}\left(p_{3}-p_{1}\right)^{\nu}\right]
$$

\footnotetext{
${ }^{1}$ There is an intermediate step that we omit for brevity, where the gluon propagator has an extra term proportional to $\left(q^{2}+\xi p^{2}\right)$. This term is dropped once we select the lightcone gauge.
} 


\section{REVIEW OF TECHNIQUES FOR CALCULATING SCATTERING AMPLITUDES}

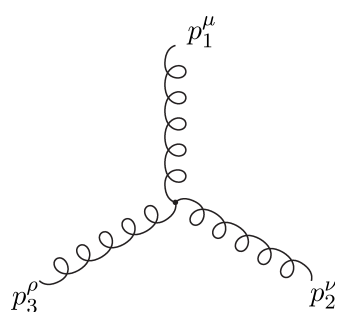

Figure 2.1: Diagrammatic form of the three-point gluon vertex where all momenta are outgoing.

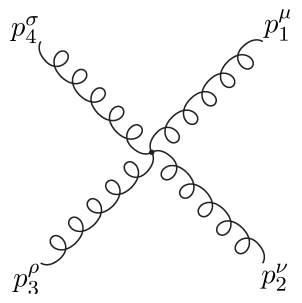

Figure 2.2: Diagrammatic form of the four-point gluon vertex where all momenta are outgoing.

$$
\begin{aligned}
V_{4}\left(p_{1}^{\mu}, p_{2}^{\nu}, p_{3}^{\rho}, p_{3}^{\sigma}\right)=i g^{2}\left[f^{a b e} f^{c d e}\left(\eta_{\mu \rho} \eta_{\nu \sigma}-\eta_{\mu \sigma} \eta_{\nu \rho}\right)\right. & +f^{a c e} f^{d b e}\left(\eta_{\mu \sigma} \eta_{\rho \nu}-\eta_{\mu \nu} \eta_{\rho \sigma}\right) \\
& \left.+f^{a d e} f^{b c e}\left(\eta_{\mu \nu} \eta_{\sigma \rho}-\eta_{\mu \rho} \eta_{\sigma \nu}\right)\right] .
\end{aligned}
$$

To calculate the scattering amplitude for a particular interaction, the polarisation vectors, $\epsilon_{\mu}^{ \pm}(p, q)$, of the external gluons must be contracted with the interaction rules and each independent Feynman diagram must be summed. This represents the traditional approach to calculating scattering amplitudes.

We present the three-gluon interaction vertex specifically for the following two reasons. We use it to derive forms for the off-shell three-point gluon vertex via axial gauge methods [122], since we will show that all tree-level amplitudes can be built from the three-gluon vertices [27, 36]. Secondly, the Feynman rule for the interaction constrains the mass dimension/momentum weight of the interaction. Combined with the helicity of the external legs, we may uniquely fix

\footnotetext{
${ }^{1}$ and subsequently on-shell three-point vertices.
} 


\subsection{Pure Yang-Mills and Pertubative Gravity}

the form of a three-point amplitudel. In this sense, we make use of the concept that a method of calculating scattering amplitudes exists via Feynman rules and Feynman diagrams only to simplify calculations avoiding their explicit use. We explore this passive use in greater detail in the subsequent chapters.

\subsubsection{Pertubative Gravity}

Gravitational theories have been shown to be intimately linked with pure YangMills, through for example the KLT relations [123].

The Einstein-Hilbert Lagrangian is written as

$$
\mathcal{L}=-\frac{2}{\kappa^{2}} \int d^{4} x \sqrt{-g} R
$$

where $R$ is the Ricci scalar, $\sqrt{-g}$ is the square root of the determinant of the metric and $\kappa=\sqrt{32 \pi G_{N}}$.

We consider the weak field approximation, when the spacetime is almost flat with small perturbations around the Minkowski metric defined by the graviton $\left|h_{\mu \nu}\right|<<1$,

$$
g_{\mu \nu}=\eta_{\mu \nu}+\kappa h_{\mu \nu}
$$

The perturbation generates an infinite series from an expansion of $\sqrt{-g}$ and the Ricci scalar. This produces an infinite series of $n$-point graviton interaction terms in the Lagrangian, where $n=2$ gives the propagator, and $n \geq 3$ are the interactions.

The calculation of scattering amplitudes requires the condition that the states enter from infinity where they are non-interacting, interact and subsequently propagate out to infinity again to a regime of non-interaction. In terms of a theory of general relativity, we can have well-defined states when the gravity force is weak and the interactions take place on a flat spacetime i.e. in the weak field approximation. One may approach the problem of calculating these interaction vertices by standard Feynman diagram techniques. However, this approach is

\footnotetext{
${ }^{1}$ specifically in pure Yang-Mills, pertubative gravity, and a particular extension to these theories.
} 


\section{REVIEW OF TECHNIQUES FOR CALCULATING SCATTERING AMPLITUDES}

plagued by large expressions and computational strain. We refer the reader to Veltman's lecture notes on the subject for a glimpse at the Feynman rules [124] as we will not directly consider that approach here. Rather, as with the Yang-Mills case, we make use of the concept that a Feynman diagram approach exists to simplify our calculations. One key takeaway from the field theory approach though, is the three-graviton interaction term is of the form [125] $h h \partial^{2} h$. Therefore it has momentum weight two, and the Feynman rules can be shown to be proportional to a product of three-point Yang-Mills Feynman rules 126,

$$
M_{3}\left(p_{\mu_{1} \mu_{2}}, p_{\nu_{1} \nu_{2}}, p_{\rho_{1} \rho_{2}}\right) \propto V_{3}\left(p_{1}^{\mu_{1}}, p_{2}^{\nu_{1}}, p_{3}^{\rho_{1}}\right) V_{3}\left(p_{1}^{\mu_{2}}, p_{2}^{\nu_{2}}, p_{3}^{\rho_{2}}\right)
$$

\subsection{Perturbation Theory and Conventions}

In this section we outline the conventions we use in labelling and describing the amplitudes present in the pertubative expansion. A pertubative expansion is permitted when the strength of the coupling constant accompanying a scattering amplitude is small. In Yang-Mills, the coupling constant is small for high energies above the QCD scale $\Lambda$. In the General Relativity, we expand in the metric when the metric describes small perturbations around a flat metric.

Within a Yang-Mills an $n$-gluon amplitude in may be expanded in the gauge coupling constant,

$$
\mathcal{A}_{n}=\delta^{(4)}\left(\sum_{i}^{n} p_{i}\right) i g^{n-2} \sum_{L \geq 0} \alpha^{L} \mathcal{A}_{n}^{(L)}
$$

where $\alpha=g^{2} c_{\Gamma}$ and the delta function ensures conservation of momentum. We define the amplitudes $\mathcal{A}_{n}^{(L)}$ to be an $n$-point, $L$-loop full colour amplitude that is short hand for

$$
\begin{aligned}
\mathcal{A}_{n}^{(L)} & \equiv \mathcal{A}_{n}^{(L)}\left(1^{h_{1}}, 2^{h_{2}}, \ldots, n^{h_{n}}\right) \equiv \mathcal{A}_{n}^{(L)}\left(\left\{T^{a_{1}}, p_{1}, \epsilon_{1}^{h_{1}}\right\},\left\{T^{a_{2}}, p_{2}, \epsilon_{2}^{h_{2}}\right\}, \ldots,\left\{T^{a_{n}}, p_{n}, \epsilon_{n}^{h_{n}}\right\}\right) \\
& \equiv \mathcal{A}_{n}^{(L)}\left(p_{1}^{h_{1}}, p_{2}^{h_{2}}, \ldots, p_{n}^{h_{n}}\right) .
\end{aligned}
$$

\footnotetext{
${ }^{1}$ The factor $c_{\Gamma}$ is defined as $\Gamma(1+\epsilon) \Gamma^{2}(1-\epsilon) / \Gamma(1-2 \epsilon) /(4 \pi)^{2-\epsilon}$.
} 
The amplitudes are a function of the group generators of the gluons, $T^{a_{i}}$, that carry the colour structure, the gluon momenta, $p_{i}$ and polarisation vectors, $\epsilon_{i}^{h_{i}}$ that have positive or negative helicity, $h_{i}= \pm$. Each amplitude is composed of helicity amplitudes that are specific assignments of all allowed helicity configurations at each loop-order and are elements of the $S$-matrix.

Gravity amplitudes have a similar prescription,

$$
\mathcal{M}_{n}^{(L)}=\delta^{(4)}\left(\sum_{i}^{n} p_{i}\right) i\left(\frac{\kappa}{2}\right)^{n-2} \sum_{L \geq 0} b^{L} M_{n}^{(L)}
$$

where $b=\left(\frac{\kappa}{2}\right)^{2} c_{\Gamma}$, and we label the stripped amplitude as

$$
\begin{aligned}
M_{n}^{L} & \equiv M_{n}^{L}\left(1^{h_{i}}, 2^{h_{2}}, \ldots, n^{h_{n}}\right) \equiv M_{n}^{(L)}\left(\left\{p_{1}, \epsilon_{1}^{h_{1}}\right\},\left\{p_{2}, \epsilon_{2}^{h_{2}}\right\}, \ldots,\left\{p_{n}, \epsilon_{n}^{h_{n}}\right\}\right) \\
& \equiv M_{n}^{L}\left(p_{1}^{h_{i}}, p_{2}^{h_{2}}, \ldots, p_{n}^{h_{n}}\right) .
\end{aligned}
$$

For both Yang-Mills and Gravity theories, calculation of scattering amplitudes by Feynman diagrams is the natural next step in this field theory approach. At $L=0$ this is cumbersome but manageable for low multiplicities. We will see shortly that through efficiency improvements given by colour ordering and the spinor helicity formalism we can adopt a recursive approach to calculating treelevel amplitudes. This method makes use of the singularity structure of the amplitudes via factorisation theorems and complex analysis. At $L \geq 1$, Feynman integrals of massless theories contain divergences at high energies (UV singularities) and at low energies (IR singularities). We regularise these divergences using dimensional regularisation [127],

$$
\int \frac{d^{4} \ell}{(2 \pi)^{4}} \frac{\mathcal{P}(\ell)}{\ell^{2}\left(\ell-p_{1}\right)^{2} \cdots\left(\ell+p_{n}\right)^{2}} \rightarrow \int \frac{d^{D} \ell}{(2 \pi)^{D}} \frac{\mathcal{P}(\ell)}{\ell^{2}\left(\ell-p_{1}\right)^{2} \cdots\left(\ell+p_{n}\right)^{2}},
$$

where $D=4-2 \epsilon$ and the divergences are shifted to singularities in the regularisation parameter $\epsilon$. Dimensional regularisation is covered in more depth in subsection 2.8.1.

The subsequent sections outline techniques that allow us to calculate these amplitudes in a more economical way. 


\section{REVIEW OF TECHNIQUES FOR CALCULATING SCATTERING AMPLITUDES}

\subsection{Colour Ordering and Group Theory Rela- tions}

Amplitudes in Yang-Mills are composed of kinematic and colour factors as can be seen from the form of the Feynman rules presented in subsection 2.1.1. Were we to calculate an amplitude from these Feynman rules we would have to consider many Feynman diagrams, each of which would be independently gauge dependent with gauge invariance only restored in the full sum. Moreover, the full sum involves a large amount of Feynman diagrams that admit cancellations culminating in a simple expression i.e. there is a great deal of redundancy in this approach. A huge step forward in simplifying the procedure came with the development of an organisation of the amplitude through its colour factors. In calculations involving the six-gluon tree-level amplitude [8, 9], the colour components were factorised from the (then called) 'subamplitudes' into traces of the group generators in the fundamental representation belonging to each external gluon line 1 . This organisation is called colour ordering and is now well established in the literature. Colour ordering can at times be taken for granted, but it has great significance in the genesis of the modern approach. Before the amplitude was organised into a sum over non-cyclic permutations of the partial amplitudes, there had been no general method of organising the amplitude into gauge invariant subsets of the Feynman diagrams that contribute to the full amplitude. However the treelevel amplitudes that multiply the colour trace structures after colour ordering are gauge invariant - the gauge invariant subsets of Feynman diagrams are those that are related by cyclic symmetry. Moreover, each independent amplitude in the colour ordering is guaranteed to be gauge invariant under a transformation in the polarisation vectors 2 [8].

$$
\epsilon_{ \pm}^{\mu}\left(p_{i}, q_{i}\right) \rightarrow \epsilon_{ \pm}^{\mu}\left(p_{i}, q_{i}\right)+c p_{j}^{\mu}
$$

where $p_{j}^{\mu}$ is the momentum of an external gluon and $c$ is a constant. With this insight, the calculation is reduced to determining the gauge invariant partial am-

\footnotetext{
${ }^{1}$ inspired by Chan-Paton factors [10] arising in string theory

2i.e. the Ward Identity.
} 


\subsection{Colour Ordering and Group Theory Relations}

plitudes.

In this section, we show how the colour ordering works in a trace basis approach for tree-level and loop-level amplitudes, showing the explicit $U\left(N_{c}\right)$ colour decomposition up to two-loops. We also explore the various symmetries and relations that each type of partial amplitude can have, including; cyclic symmetry, flip symmetry and group theory relations including decoupling identities. We dedicate subsection 2.3.2 to summarising decoupling identities [128] and the group theory relations [15, 16] that relate partial amplitudes to each other. In doing so, we take a cursory glance at alternative methods of colour decomposition and note their advantages.

\subsubsection{Colour Ordering}

Yang-Mills is invariant under gauge transformations described by the gauge group $S U\left(N_{c}\right)$. In terms of the colour factors we have met in the Feynman rules, this amounts to writing the generators of the external gluons in a representation of $S U\left(N_{c}\right)$. We are free to extend $S U\left(N_{c}\right)$ to $U\left(N_{c}\right)$ as $U\left(N_{c}\right)=S U\left(N_{c}\right) \times U(1)$.

Working in $U\left(N_{c}\right)$ effectively amounts to working in $S U\left(N_{c}\right)$ since the $U(1)$ 'photon' does not couple to the gluons. Therefore, the only physical partial amplitudes that are present in $U\left(N_{c}\right)$ are those that arise in the $S U\left(N_{c}\right)$ theory as well. The remaining non-physical $U\left(N_{c}\right)$ amplitudes are only present in order to ensure that when a $U(1)-$ 'photon' is introduced into the theory, the partial amplitudes that survive, sum to give a vanishing contribution to the amplitude. If the amplitudes are non-physical it is natural to wonder about their use. The reasoning is two-fold:

- the colour algebra for $U\left(N_{c}\right)$ theories is simpler to perform,

- the vanishing sums of partial amplitudes when including $U(1)$-'photons' into the theory source the decoupling identities that we use as checks.

Each gluon has an associated Hermitian group generator $T^{a}$, where the generators belong to either $U\left(N_{c}\right)$ or $S U\left(N_{c}\right)$ as appropriate. The unitary group $U\left(N_{c}\right)$ 


\section{REVIEW OF TECHNIQUES FOR CALCULATING SCATTERING AMPLITUDES}

has dimension $N_{c}^{2}$ whilst the special unitary group $S U\left(N_{c}\right)$ has dimension $N^{2}-1$ and the group generators span the colour space. The index $a$ labels each of the generators and in the fundamental representation $a=1, \ldots, N^{2}$ for $U\left(N_{c}\right)$ and $a=1, \ldots, N^{2}-1$ for $S U\left(N_{c}\right)$. Both $U\left(N_{c}\right)$ and $S U\left(N_{c}\right)$ are non-abelian groups described by the commutation relation,

$$
\left[T^{a}, T^{b}\right]=i f^{a b c} T^{c}
$$

where $f^{a b c}$ are the non-trivial structure constants. We normalise the generators according to

$$
\operatorname{Tr}\left(T^{a} T^{b}\right)=\delta^{a b}
$$

The colour decomposition follows from considering a particular diagram written in terms of three-point vertices where one of the legs labels an internal line. Each three-point vertex has a factor of $f^{a b c}$ associated with it. These structure constants can be rewritten in terms of traces of the gluon generators, using the commutation relation of the gluon generators resulting in

$$
i f^{a b c}=\operatorname{Tr}\left(T^{a} T^{b} T^{c}\right)-\operatorname{Tr}\left(T^{c} T^{b} T^{a}\right) .
$$

An internal line will have its generator appear in two structure constants and subsequently in the product of traces. By combining the traces using a Fierz identity the colour decomposition can be achieved. In order to obtain the amplitude in $S U\left(N_{c}\right)$, we could apply the $S U\left(N_{c}\right)$ Fierz identity:

$$
\sum_{a} T_{i j}^{a} T_{k l}^{a}=\delta_{i l} \delta_{j k}-\frac{1}{N_{c}} \delta_{i j} \delta_{k l}
$$

However we choose to obtain the amplitude in $U\left(N_{c}\right)$ using the $U\left(N_{c}\right)$ form of the Fierz identity:

$$
\sum_{a} T_{i j}^{a} T_{k l}^{a}=\delta_{i l} \delta_{j k}
$$

The following trace structures are all the possible combinations of traces shar- 


\subsection{Colour Ordering and Group Theory Relations}

ing the same generator that can appear in a colour decomposition. Using the Fierz identity with our normalisation of the generators we have,

$$
\begin{aligned}
& \operatorname{Tr}\left(T^{a} X\right) \operatorname{Tr}\left(T^{a} Y\right)=T_{i j}^{a} X_{j i} T_{k l}^{a} Y_{l k}=T_{i j}^{a} T_{k l}^{a} X_{j i} Y_{l k}=\delta_{i l} \delta_{j k} X_{j i} Y_{l k}=X_{j i} Y_{i j}=\underset{(2.28)}{\operatorname{Tr}(X Y),} \\
& \operatorname{Tr}\left(T^{a} X T^{a} Y\right)=T_{i j}^{a} X_{j k} T_{k l}^{a} Y_{l i}=T_{i j}^{a} T_{k l}^{a} X_{j k} Y_{l i}=\delta_{i l} \delta_{j k} X_{j k} Y_{l i}=X_{j j} Y_{i i}=\operatorname{Tr}(X) \operatorname{Tr}(Y),
\end{aligned}
$$

and

$\operatorname{Tr}\left(X T^{a} T^{a} Y\right)=X_{i j} T_{j k}^{a} T_{k l}^{a} Y_{l i}=T_{j k}^{a} T_{k l}^{a} X_{i j} Y_{l i}=\delta_{j l} \delta_{k k} X_{i j} Y_{l i}=X_{i j} Y_{j i}=N_{c} \operatorname{Tr}(X Y)$

Repeated application of the previous formulae lead to the expression of the $n$-point tree amplitude in a colour trace basis as [8, 9]

$$
\mathcal{A}_{n}^{(0)}(1,2,3, \cdots, n)=\sum_{S_{n} / Z_{n}} \operatorname{Tr}\left[T^{a_{1}} \cdots T^{a_{n}}\right] A_{n}^{(0)}\left(a_{1}, a_{2}, \cdots a_{n}\right)
$$

The sum is over all non-cyclic permutations of the legs, defined by the quotient group $S_{n} / Z_{n}$. This group is composed of $S_{n}$, the permutation group of $n$ objects and the cyclic group $Z_{n}$. Every partial amplitude in the colour decomposition shares the same symmetry as the traces they multiply. In the tree-level case we have what we define to be single traces, i.e. the group generators are contained in one trace. Since a trace is symmetric under a cycling of its elements, the partial amplitude is invariant under a cycling of the external legs as well,

$$
A_{n}^{(0)}\left(a_{1}, a_{2}, \ldots, a_{n}\right)=A_{n}^{(0)}\left(a_{2}, \ldots, a_{n}, a_{1}\right)
$$

Hence, performing the colour ordering allows us to reduce the amount of diagrams that are required to describe a tree-level amplitude because we can relate $n$ of the amplitudes by cyclic symmetry. After taking into account this cyclic symmetry, the number of independent diagrams is $n ! / n=(n-1) !$ - all the non-cyclic permutations of the external legs. 


\section{REVIEW OF TECHNIQUES FOR CALCULATING SCATTERING AMPLITUDES}

Other non-trivial relations exist that reduce the number of independent partial amplitudes. Although we don't make use of them in this thesis, the developments should be taken note of. The Kleiss-Kuijf relations [129] reduce the number of independent diagrams to $(n-2)$ ! and the BCJ relations [130] that reduce this further to $(n-3)$ !.

The colour ordering procedure simplifies the game if one were wish to proceed using Feynman diagrams. The three-gluon and four-gluon Feynman rules are stripped of their colour factors and are given by ${ }^{\mathrm{t}}$,

$$
\begin{aligned}
&\left.V_{3}\left(p_{1}^{\mu}, p_{2}^{\nu}, p_{3}^{\rho}\right)\right|_{\text {c.o. }}=\left[\eta^{\mu \nu}\left(p_{1}-p_{2}\right)^{\rho}+\eta^{\nu \rho}\left(p_{2}-p_{3}\right)^{\mu}+\eta^{\rho \mu}\left(p_{3}-p_{1}\right)^{\nu}\right], \\
&\left.V_{4}\left(p_{1}^{\mu}, p_{2}^{\nu}, p_{3}^{\rho}, p_{3}^{\sigma}\right)\right|_{\text {c.o. }}=\left[2 \eta^{\mu \rho} \eta^{\nu \sigma}-\eta^{\mu \nu} \eta^{\rho \sigma}-\eta^{\mu \sigma} \eta^{\nu \rho}\right] .
\end{aligned}
$$

This form of the Feynman rules highlights another property. The tree-level partial amplitudes are also invariant under a reflection identity, hereto referred to as a flip symmetry defined by

$$
A_{n}^{(0)}\left(a_{1}, a_{2}, \ldots, a_{n}\right)=(-1)^{n} A_{n}^{(0)}\left(a_{n}, \ldots, a_{2}, a_{1}\right)
$$

This follows from the anti-symmetry of the colour ordered Feynman Rules.

Since the full amplitude is gauge invariant and we have separated the amplitude into linearly independent partial amplitudes, each of the independent partial amplitudes must be gauge invariant. With this formulation we ensure that in the construction of the full amplitude, we are working with gauge invariant subsets of the full amplitude. By identifying the gauge invariant subsets of the amplitude we avoid large cancellations that would occur from the Feynman diagram approach, where each Feynman Diagram is not gauge invariant and gauge invariance is only recovered in the full sum. This is a core principle of the modern amplitudes approach.

\footnotetext{
${ }^{1}$ We have removed a factor of $i$ from the vertices due to the convention set out in (2.17).
} 
At one-loop, we can play the same games to decompose the amplitude into colour and kinematic factors. In a $U\left(N_{c}\right)$ gauge theory the one-loop $n$-point amplitude can be expanded as [128],

$$
\begin{aligned}
& \mathcal{A}_{n}^{(1)}(1,2,3, \cdots, n)=\sum_{S_{n} / Z_{n}} N_{c} \operatorname{Tr}\left[T^{a_{1}} \cdots T^{a_{n}}\right] A_{n: 1}^{(1)}\left(a_{1}, a_{2}, \cdots a_{n}\right) \\
& +\sum_{r=2}^{[n / 2]+1} \sum_{S_{n} /\left(Z_{r-1} \times Z_{n+1-r}\right)} \operatorname{Tr}\left[T^{b_{1}} \cdots T^{b_{r-1}}\right] \operatorname{Tr}\left[T^{c_{r}} \cdots T^{c_{n}}\right] A_{n: r}^{(1)}\left(b_{1} \cdots b_{r-1} ; c_{r} \cdots c_{n}\right) .
\end{aligned}
$$

For $n$ even and $r=n / 2+1$ there is an extra $Z_{2}$ modded out of the summation to ensure each colour structure only appears once. At leading order in the number of colours $N_{c}$, we have the familiar single trace structures. In the large $N_{c}$ limit [131] these diagrams dominate and lead to a planar theory. We can gain an intuition for this planarity by examining the colour structures appearing at subleading orders in colour too. Double trace structures appear here, where the group generators are split across the product of two traces. The difference between these colour structures is clearly shown diagrammatically by colour dressing the partial amplitudes.
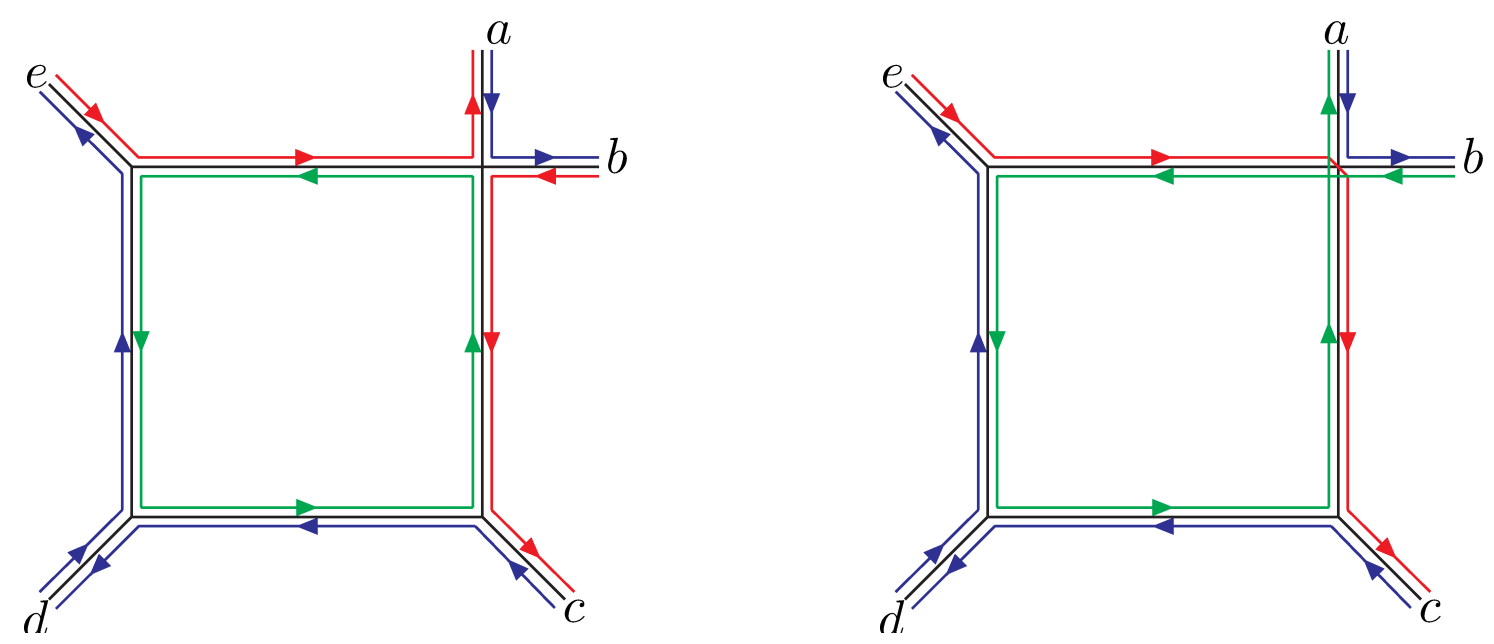

Figure 2.3: Colour dressed one-mass box diagrams, demonstrating how the trace structures $N_{c} \operatorname{Tr}(a b c d e)$ and $\operatorname{Tr}(a b) \operatorname{Tr}(c d e)$ can arise in a one-loop amplitude. 


\section{REVIEW OF TECHNIQUES FOR CALCULATING SCATTERING AMPLITUDES}

The leading in colour partial amplitude $A_{5: 1}^{(1)}$ is planar as there are no colour lines that cross. The subleading in colour amplitude has a non-planar structure owing to the crossing colour lines. This is the distinction we make between planar and non-planar amplitudes.

The leading in colour partial amplitudes share the same symmetry as the tree-level partial amplitudes, (2.32) and (2.35), while the sum is again over all non-cyclic permutations of all the external legs.

The double trace structures of the subleading in colour partial amplitudes also reflect the symmetries of the partial amplitudes. If we consider separating set of the all the external gluons of the partial amplitude into the two sets $\left\{b_{1}, \ldots, b_{r-1}\right\}$ and $\left\{c_{r}, \ldots, c_{n}\right\}$ defined by

$$
\operatorname{Tr}\left[T^{b_{1}} \cdots T^{b_{r-1}}\right] \operatorname{Tr}\left[T^{c_{r}} \cdots T^{c_{n}}\right] A_{n: r}^{(1)}\left(b_{1} \cdots b_{r-1} ; c_{r} \cdots c_{n}\right) .
$$

The subleading partial amplitudes exhibit cyclic invariance under a cycling of the two sets individually. Additionally, the partial amplitudes have a similar flip symmetry to the planar case,

$$
A_{n: r}^{(1)}\left(b_{1}, b_{2} \ldots, b_{r-1} ; c_{r}, c_{r+1}, \ldots, c_{n}\right)=(-1)^{n} A_{n: r}^{(1)}\left(b_{1}, b_{r-1}, \ldots, b_{2} ; c_{r}, c_{n}, \ldots, c_{r+1}\right),
$$

where both sets are flipped simultaneously. For the special case, $r=3$, we are free to flip the sets independently. This is due to the fact that a flip of a two element set is equivalent to the cyclic reordering of such a set.

The partial amplitudes $A_{n: 2}^{(1)}$ are the only unphysical partial amplitudes present in the $U\left(N_{c}\right)$ colour decomposition at one-loop. They are absent (or zero) in the $S U\left(N_{c}\right)$ case since the generators of $S U\left(N_{c}\right)$ are traceless.

Two-loop amplitudes are the only other class of amplitudes we will consider

in this thesis. A general two-loop amplitude may be expanded in a $U\left(N_{c}\right)$ colour 


\subsection{Colour Ordering and Group Theory Relations}

trace basis as,

$$
\begin{aligned}
& \mathcal{A}_{n}^{(2)}(1,2, \cdots, n)=N_{c}^{2} \sum_{S_{n} / Z_{n}} \operatorname{Tr}\left[T^{a_{1}} T^{a_{2}} \cdots T^{a_{n}}\right] A_{n: 1}^{(2)}\left(a_{1}, a_{2}, \cdots, a_{n}\right) \\
& +N_{c} \sum_{r=2}^{[n / 2]+1} \sum_{S_{n} /\left(Z_{r-1} \times Z_{n+1-r}\right)} \operatorname{Tr}\left[T^{b_{1}} T^{b_{2}} \cdots T^{b_{r-1}}\right] \operatorname{Tr}\left[T^{c_{r}} \cdots T^{c_{n}}\right] A_{n: r}^{(2)}\left(b_{1}, b_{2}, \cdots, b_{r-1} ; c_{r} \cdots c_{n}\right) \\
& +\sum_{s=1} \sum_{t=s}^{[n / 3]} \sum_{S_{n} /\left(Z_{s} \times Z_{t} \times Z_{n-s-t}\right)} \operatorname{Tr}\left[T^{d_{1}} \cdots T^{d_{s}}\right] \operatorname{Tr}\left[T^{e_{s+1}} \cdots T^{e_{s+t}}\right] \operatorname{Tr}\left[T^{f_{s+t+1}} \cdots T^{f_{n}}\right] \\
& +\sum_{S_{n} / Z_{n}} \operatorname{Tr}\left[T^{a_{1}} T^{a_{2}} \cdots T^{a_{n}}\right] A_{n: 1 B}^{(2)}\left(a_{1}, a_{2}, \cdots, a_{n}\right) .
\end{aligned}
$$

Again, for $n$ even and $r=n / 2+1$ there is an extra $Z_{2}$ modded out of the summation to ensure each colour structure only appears once. In the $s, t$ summations there is an extra $Z_{2}$ when exactly two of $s, t$ and $n-s-t$ are equal and an extra $S_{3}$ when all three are equal.

This amplitude has three different orders in colour, two of which we have already seen. Triple trace structures appear at sub-subleading in colour. Subsubleading in colour is composed of two colour structures, $A_{n: s, t}^{(2)}$ and a single trace structure $A_{n: 1 B}^{(2)}$. Despite the fact $A_{n: 1 B}^{(2)}$ multiplies a single trace, this is a non-planar amplitude. In chapter 4 we calculate the full colour two-loop fivepoint all-plus amplitude and calculate this partial amplitude explicitly. Part of this partial amplitude is a rational piece that can be calculated by considering fig. 2.4, where it is clear that this is a non-planar contribution but still has a single trace colour structure.

The two-loop partial amplitudes exhibit generalised versions of the cyclic and flip symmetries already presented. At two-loops however, the full amplitude cannot be determined by decoupling identities alone. There exist additional group theory identities [15, 16] that are discussed at the end of the next section. 


\section{REVIEW OF TECHNIQUES FOR CALCULATING SCATTERING AMPLITUDES}

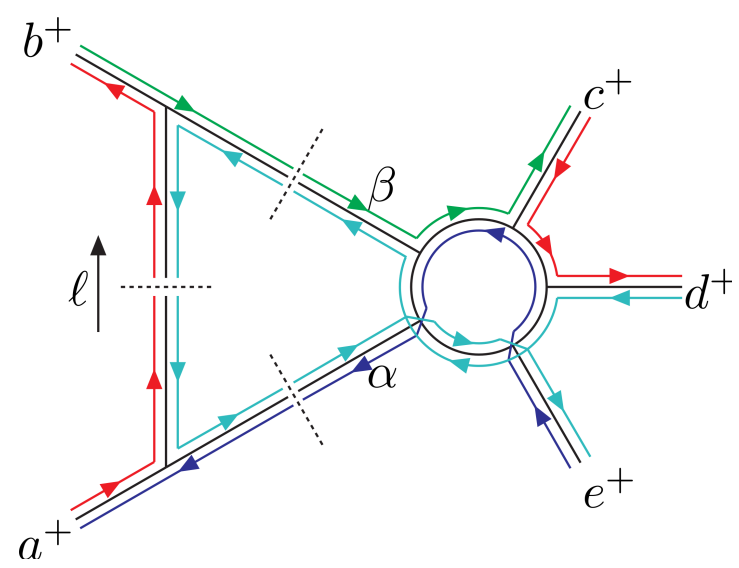

Figure 2.4: The 'maximally non-factorising' sub-subleading rational contribution to the full colour two-loop five-gluon amplitude, which multiplies the trace structure $N_{c}^{0} \operatorname{Tr}(a b c d e)$.

\subsubsection{Decoupling Identities}

Using $U\left(N_{c}\right)$ as the gauge group for Yang-Mills provides additional constraints on the physical and unphysical partial amplitudes in the colour decomposition. Setting any of the generators of the gauge bosons in the amplitude to be the $U(1)$ generator

$$
T^{a_{i}} \rightarrow T^{U(1)_{i}}=\frac{1}{\sqrt{N_{c}}} \mathbb{1}
$$

where $i$ labels any of the external $\operatorname{legs} i \in\{1, \ldots, n\}$, we obtain traces whose product is a sum of partial amplitudes. The sum is the contribution to an unphysical amplitude containing a $U(1)$-'photon' therefore it should vanish. The vanishing sum arising from the various ways of setting the gluon generators to be the 'photon' gives the decoupling identities [128].

As a short example, take a four point tree-level amplitude whose colour decomposition is,

$$
\mathcal{A}_{4}^{(0)}=\sum_{S_{n} / Z_{n}} \operatorname{Tr}\left[T^{a_{1}} T^{a_{2}} T^{a_{3}} T^{a_{4}}\right] A_{4}^{(0)}(1,2,3,4) .
$$

Setting $T^{a_{1}}$ to be the $U(1)$ generator reduces the traces to a common trace with 


\subsection{Colour Ordering and Group Theory Relations}

the vanishing coefficient

$$
\frac{1}{\sqrt{N_{c}}} \operatorname{Tr}\left[T^{a_{2}} T^{a_{3}} T^{a_{4}}\right]\left(A_{4}^{(0)}\left(a_{1}, a_{2}, a_{3}, a_{4}\right)+A_{4}^{(0)}\left(a_{2}, a_{1}, a_{3}, a_{4}\right)+A_{4}^{(0)}\left(a_{2}, a_{3}, a_{1}, a_{4}\right)\right) .
$$

This vanishing coefficient gives the decoupling identity,

$$
A_{4}^{(0)}\left(a_{1}, a_{2}, a_{3}, a_{4}\right)+A_{4}^{(0)}\left(a_{2}, a_{1}, a_{3}, a_{4}\right)+A_{4}^{(0)}\left(a_{2}, a_{3}, a_{1}, a_{4}\right)=0,
$$

with the extension to $n$-point given by,

$$
A_{n}^{(0)}(1,2,3, \ldots, n)+A_{n}^{(0)}(2,1,3,4, \ldots, n)+A_{n}^{(0)}(2,3,1, \ldots, n)+\ldots+A_{n}^{(0)}(2,3, \ldots, 1, n)=0
$$

This is identical to the dual Ward identities found and discussed in [8, 11]. We will refer to this a a tree-level decoupling identity as these types of decoupling identities also appear at loop-level.

At one-loop there are additional decoupling identities that are available from taking one or more of the generators of the gauge bosons to be $U(1)$-'photons'.

Before continuing, in the colour decompositions at loop-level there is a subtlety regarding our definition of the labels of the external legs that can cause an issue when discussing decoupling identities. At one-loop, for subleading orders in colour we use the sets $\left\{b_{i}\right\} \cup\left\{c_{i}\right\} \in\left\{p_{1}, p_{2}, \ldots, p_{n}\right\}$ to distinguish between the gauge bosons whose generators appear in a double trace. Whereas we use $a_{i} \in\left\{p_{1}, p_{2}, \ldots, p_{n}\right\}$ to label the gauge bosons whose generators appear in the single trace terms. When we use numbers by themselves, i.e. $(1,2,3,4,5)$ we are referring to an explicit choice, i.e. $a_{1}=p_{1}$, etc. This explicit choice will arise when evaluating the sums in the colour decomposition. Consequently, there are instances when for example $b_{1}=a_{1}$ and $c_{i}=a_{i}$ for $i=2, \ldots, n$.

Using this assignment in the one-loop decomposition of (2.36) for $n=5$ we may relate leading and subleading partial amplitudes.

Taking leg $a_{1}$ to be the $U(1)$ leg and extracting the coefficient of $\operatorname{Tr}\left[T^{a_{2}} T^{a_{3}} T^{a_{4}} T^{a_{5}}\right]$ 


\section{REVIEW OF TECHNIQUES FOR CALCULATING SCATTERING AMPLITUDES}

implies

$$
\begin{aligned}
N_{c}\left(A_{5: 2}^{(1)}\left(b_{1} ; c_{2}, c_{3}, c_{4}, c_{5}\right)\right. & +A_{5: 1}^{(1)}\left(a_{1}, a_{2}, a_{3}, a_{4}, a_{5}\right)+A_{5: 1}^{(1)}\left(a_{2}, a_{1}, a_{3}, a_{4}, a_{5}\right) \\
& \left.+A_{5: 1}^{(1)}\left(a_{2}, a_{3}, a_{1}, a_{4}, a_{5}\right)+A_{5: 1}^{(1)}\left(a_{2}, a_{3}, a_{4}, a_{1}, a_{5}\right)\right)=0 .
\end{aligned}
$$

Thus the $A_{5: 2}^{(1)}$ are expressible in terms of the leading in colour amplitudes.

Another type of subleading partial amplitude, $A_{5: 3}^{(1)}$, is related to $A_{5: 1}^{(1)}$ and $A_{5: 2}^{(1)}$ by setting legs $a_{1}$ and $a_{2}$ to be the $U(1)$ legs. Only one trace structure survives from the $A_{5: 3}^{(1)}$, s, i.e. $\operatorname{Tr}\left[T^{b_{1}} T^{b_{2}}\right] \operatorname{Tr}\left[T^{c_{3}} T^{c_{4}} T^{c_{5}}\right]$ (when $a_{i}=b_{j}$ for $i, j=1,2$ ) which reduces to $\operatorname{Tr}\left[T^{a_{3}} T^{a_{4}} T^{a_{5}}\right]$. Both the $A_{5: 2}^{(1)}$ 's and $A_{5: 1}^{(1)}$ 's will contribute to this decoupling identity, relating all three types of trace structures at one-loop. Using (2.45) the $A_{5: 3}^{(1)}$ partial amplitude is expressed in terms of the leading in colour partial amplitude. This extends to $n$-point via repeated application of the decoupling identities. All the $A_{n: r}^{(1)}$ can be expressed as sums over the $A_{n: 1}^{(1)}$ [128,

$$
A_{n ; r}^{(1)}\left(b_{1}, b_{2}, \ldots, b_{r-1} ; c_{r}, c_{r+1}, \ldots, c_{n}\right)=(-1)^{r-1} \sum_{\sigma \in C O P\{\alpha\}\{\beta\}} A_{n ; 1}^{(1)}(\sigma)
$$

where五 $\alpha_{i} \in\{\alpha\} \equiv\left\{b_{r-1}, b_{r-2}, \ldots, b_{2}, b_{1}\right\}$ and $\beta_{i} \in\{\beta\} \equiv\left\{c_{r}, c_{r+1}, \ldots, c_{n-1}, c_{n}\right\}$.

$C O P\{\alpha\}\{\beta\}$ is the set of all permutations of $\{1,2, \ldots, n\}$ with $n$ held fixed that preserve the cyclic ordering of the $\alpha_{i}$ within $\{\alpha\}$ and of the $\beta_{i}$ within $\{\beta\}$, while allowing for all possible relative orderings of the $\alpha_{i}$ with respect to the $\beta_{i}$. For example if $\{\alpha\}=\{2,1\}$ and $\{\beta\}=\{3,4,5\}$, then $C O P\{\alpha\}\{\beta\}$ contains the twelve elements
$(2,1,3,4,5)$,
$(2,3,1,4,5)$
$(2,3,4,1,5)$
$(3,2,1,4,5)$,
$(3,2,4,1,5)$
$(3,4,2,1,5)$,
$(1,2,3,4,5)$,
$(1,3,2,4,5)$
$(1,3,4,2,5)$,
$(3,1,2,4,5)$,
$(3,1,4,2,5)$,
$(3,4,1,2,5)$.

These decoupling identities completely determine the subleading partial amplitudes in terms of the leading partial amplitudes. In chapter 4 following on from work presented in [89], we present more compact forms of the subleading

\footnotetext{
${ }^{1}$ Note that the ordering of the first set of indices is reversed with respect to the second.
} 


\subsection{Colour Ordering and Group Theory Relations}

in colour amplitudes deduced from momentum weight and spinor weight/little group scaling. These topics are discussed in section 2.5.

At two-loop, the decoupling identities cannot be used to determine each of the subleading in colour partial amplitudes uniquely. Instead they can be used to uniquely determine the specifically $U\left(N_{c}\right)$ functions. In section 4.2 we will see explicit examples in the context of the two-loop five-gluon amplitude.

Additionally, since we're specifying generators of the gauge bosons to be the $U(1)$ generator, we are introducing powers in the number of colours $N_{c}$. This means that decoupling identities are present at different orders in $N_{c}$ and will therefore be independent, even if they have matching trace structures. An important example is the case, $T^{a_{1}} \rightarrow T^{U(1)}$. Two of the resultant decoupling identities involve a tree-like decoupling identity. One at leading in colour,

$$
N_{c}^{\frac{3}{2}}\left(A_{n: 1}^{(2)}(1,2, \ldots, n)+A_{n: 1}^{(2)}(2,1, \ldots, n)+\ldots+A_{n: 1}^{(2)}(2, \ldots, 1, n)\right)=0,
$$

and another occurring at sub-subleading order,

$$
N_{c}^{-\frac{1}{2}}\left(A_{n: 1 B}^{(2)}(1,2, \ldots, n)+A_{n: 1 B}^{(2)}(2,1, \ldots, n)+\ldots+A_{n: 1 B}^{(2)}(2, \ldots, 1, n)\right)=0 .
$$

These two decoupling identities cannot be associated since they differ by a factor of $N_{c}^{2}$. In fact, the sub-subleading in colour partial amplitude cannot be related to any other partial amplitude through decoupling identities at all. This poses a problem when checking a direct calculation since this decoupling identity does not specify the $A_{n: 1 B}^{(2)}$ uniquely. Having a relation including other partial amplitudes is a much stronger condition and is far more desirable. First, we must review alternative colour decompositions, since this extra constraint arises from comparing the trace based colour decomposition to another decomposition.

There are alternative colour decompositions available that are advantageous in different scenarios. Zeppenfeld [12] introduced a complete orthogonal basis of colour factors, thus simplifying cross-section calculations. Dixon, Del Duca and Maltoni [13, 14] write the amplitude in terms of the structure constants $f^{a b c}$. In this form the decoupling identities are made manifest as any structure constant 


\section{REVIEW OF TECHNIQUES FOR CALCULATING SCATTERING AMPLITUDES}

containing a $U(1)$-'photon' will automatically vanish since the $U(1)$ generator commutes with all other generators. This method of colour decomposition has many advantages. It has the same qualities as the trace based decomposition, but in addition, the decoupling identities and the Kleiss-Kuijf relations are made manifest. The amplitude is written only in terms of its independent partial amplitudes.

Examining the link between two colour decompositions, the trace based colour decomposition [8, 9] and the colour based decomposition [13, 14] in $S U\left(N_{c}\right)$ sheds light on additional group theory constraints on the partial amplitudes. This method was utilised at four-point [15] and five-point [16] to derive these group theory relations up to $L$-loops. It is the latter result that we make use of in chapter 4 to provide the additional consistency check on the sub-subleading in colour single trace partial amplitude. The key element of the method is noticing that the trace based decomposition has a larger dimension than the number of independent colour factors in the colour based decomposition. A transformation matrix can be used to describe the relation between two decompositions and since the colour based composition has a smaller dimension, the transformation matrix will contain eigenvectors that span the kernel of the linear map. It is the action of the partial amplitudes on these null vectors that gives the decoupling identities [128], Kleiss-Kuijf relations [129], BCJ relations [130] and give additional group theory relations between leading and subleading partial amplitudes.

For the two-loop five-gluon amplitude, we utilise the methods of [15, 16] to find such a relation, this is discussed in section 4.2.

\subsubsection{Summary}

These colour relations are used in all subsequent calculations of gluon amplitudes. Separating out the colour from the kinematics reduces the problem of calculating a gluon amplitude down to deducing the individual gauge invariant partial amplitudes. The colour decomposition illuminates symmetries of the partial amplitudes aiding as a calculational tool.

Despite the usefulness of the DDM formulation, we choose to use the tracebased decomposition in $U\left(N_{c}\right)$ Yang-Mills, but with the main focus of obtaining 
compact forms for the physical $S U\left(N_{c}\right)$ partial amplitudes. With that, we turn out attention from the colour to the kinematics - the partial amplitudes themselves.

\subsection{Spinor Helicity Formalism}

The spinor helicity formalism is an essential tool in a particle physicists kit when studying scattering amplitudes. First seen in the context of QED calculations 20 25] and later extended to non-abelian gauge theories by [26] these developments allowed the polarisation vectors of gluons to be written in terms of the spinor helicity formalism. This formalism has the advantage of encoding the massless condition of the four-vectors, the orthogonality of the gluon polarisations and leads to compact expressions for the kinematic components of amplitudes. The formalism essentially trades on-shell Minkowski four-vectors that transform under $S O^{+}(1,3)$, for a pair of Weyl spinors, $\lambda$ and $\bar{\lambda}$, that transform under $S L(2, \mathbb{C})$, the universal cover of $\mathrm{SO}^{+}(1,3)$. That is, the 4-dimensional Dirac representation of the Lorentz group is reducible, to a 2-dimensional Weyl representation. We will see that this decomposition into $\lambda$ 's and $\bar{\lambda}$ 's is the most natural way of expressing amplitudes as, for example, the tree-level MHV amplitude is solely expressed in terms of the $\lambda$ spinors. Amplitudes containing massless fermions can also be expressed using the same formalism. Since we are working in the high energy regime of Yang-Mills, the fermions would be ultra-relativistic and thus behave similarly to massless particles.

In this section we introduce the spinor helicity formalism. We show how the four momentum of a massless particle is naturally expressed as a bispinor and give its decomposition into a product of two Weyl spinors. Additionally, we define the products of these spinors in terms of the angled and square brackets that are synonymous with the formalism, subsequently building Lorentz invariant products from them.

We begin by stating our conventions for the gamma matrices, $\gamma^{\mu}$. we choose 


\section{REVIEW OF TECHNIQUES FOR CALCULATING SCATTERING AMPLITUDES}

a convenient representation - the Weyl representation,

$$
\gamma^{\mu}=\left(\begin{array}{cc}
0 & \sigma^{\mu} \\
\bar{\sigma}^{\mu} & 0
\end{array}\right)
$$

where $\sigma^{\mu} \equiv\left(\mathbb{1}, \sigma^{i}\right)$ and $\bar{\sigma}^{\mu} \equiv\left(\mathbb{1},-\sigma^{i}\right)$. Writing a four momentum using the gamma matrices in this representation, using the usual combination $\gamma^{\mu} \partial_{\mu}$,

$$
\not p=\left(\begin{array}{cc}
0 & p_{\alpha \dot{\beta}} \\
p_{\dot{\alpha} \beta} & 0
\end{array}\right)
$$

highlights the property that momenta transform under the reducible $\left(\frac{1}{2}, \frac{1}{2}\right)$ representation of the Lorentz group since this representation is block diagonal. From this perspective we see that the four momentum is more naturally defined from a bispinor $p_{\alpha \dot{\beta}}$, where we have,

$$
p_{\mu} \sigma_{\alpha \dot{\beta}}^{\mu}=p_{\alpha \dot{\beta}}=\left(\begin{array}{cc}
-p^{0}+p^{3} & p^{1}-i p^{2} \\
p^{1}+i p^{2} & -p^{0}-p^{3}
\end{array}\right)
$$

The determinant of this matrix gives the mass-shell condition,

$$
\operatorname{det}\left(p_{\alpha \dot{\beta}}\right)=p_{0}^{2}-p_{i}^{2}=p_{\mu} p^{\mu}=m^{2} .
$$

For massless particles the determinant of this matrix is zero and we can decompose the four-vector into an outer product of two, two-component Weyl spinors,

$$
p_{\alpha \dot{\beta}}=\left(\begin{array}{c}
\lambda_{1} \\
\lambda_{2}
\end{array}\right)\left(\begin{array}{ll}
\bar{\lambda}_{1} & \bar{\lambda}_{2}
\end{array}\right)=\lambda_{\alpha} \bar{\lambda}_{\dot{\beta}}
$$

These spinors satisfy the Weyl equation and for real momenta the solutions are given by,

$$
\lambda_{\alpha}=\frac{t}{\sqrt{p_{0}-p_{3}}}\left(\begin{array}{c}
p_{0}-p_{3} \\
-p_{1}-i p_{2}
\end{array}\right)
$$


and

$$
\bar{\lambda}_{\dot{\beta}}=\frac{t^{-1}}{\sqrt{p_{0}-p_{3}}}\left(p_{0}-p_{3}, \quad-p_{1}+i p_{2}\right)
$$

The reality condition on these spinors implies $\lambda_{\alpha}=\operatorname{sign}\left(p_{0}\right)\left(\bar{\lambda}_{\dot{\beta}}\right)^{*}$, however following the insight of Witten [27] we may also take $p_{i} \in \mathbb{C}$ such that $\lambda_{\alpha} \neq$ $\operatorname{sign}\left(p_{0}\right)\left(\bar{\lambda}_{\dot{\beta}}\right)^{*}$ and the spinors are independent of each other. This is an immensely important quality of the spinors, as it allows us to write non-vanishing three-point amplitudes. We make use of complex momenta throughout this thesis and unless otherwise stated, all momenta should be taken to be complex.

The free parameter $t$, in the solutions, is related to the Little Group scaling - what we refer to in this thesis as spinor weight. We are free to scale the Weyl spinors in this fashion since it leaves the four-momentum invariant. This is investigated further in subsection 2.4.1.

The spinor indices are manipulated through the use of the Levi-Civita tensor,

$$
\begin{gathered}
\epsilon^{\alpha \beta}=-i \sigma_{2}^{\alpha \beta}, \\
\lambda_{\alpha} \epsilon^{\alpha \beta}=\lambda^{\beta} .
\end{gathered}
$$

Spinor products of the $\lambda$ 's or $\bar{\lambda}$ 's can also be defined through the use of the Levi-Civita tensor. Given two momenta, $p_{1}$ and $p_{2}$ defined using (2.53),

$$
\left\langle p_{1} p_{2}\right\rangle \equiv\langle 12\rangle=\epsilon_{\alpha \beta} \lambda_{1}^{\alpha} \lambda_{2}^{\beta}, \quad\left[p_{1} p_{2}\right] \equiv[12]=\epsilon_{\dot{\alpha} \dot{\beta}} \bar{\lambda}_{1}^{\dot{\alpha}} \bar{\lambda}_{2}^{\dot{\beta}}
$$

Any physical quantity of interest should be Lorentz invariant and so it is convenient for us to construct Lorentz invariant products of these spinor variables. The familiar Mandlestam invariants are defined using these spinorial products as,

$$
s_{i j}=\left(p_{i}+p_{j}\right)^{2}=2 p_{i} \cdot p_{j}=\langle i j\rangle[j i]
$$

for two particles. We define general Mandlestam invariants for the squared sum 


\section{REVIEW OF TECHNIQUES FOR CALCULATING SCATTERING AMPLITUDES}

of an arbitrary number of gluon momenta as,

$$
t_{i \ldots j}=p_{i \ldots j}^{2}=\left(p_{i}+\ldots+p_{j}\right)^{2} .
$$

Another Lorentz invariant quantity arises in the expression of contractions of momenta with the four dimensional Levi-Civita tensor,

$$
\varepsilon(i, j, k, l)=\varepsilon_{\mu \nu \rho \sigma} p_{i}^{\mu} p_{j}^{\nu} p_{k}^{\rho} p_{l}^{\sigma}=\operatorname{tr}_{+}\left(p_{i} p_{j} p_{k} p_{l}\right)-\operatorname{tr}_{-}\left(p_{i} p_{j} p_{k} p_{l}\right)
$$

where

$$
\begin{aligned}
& \operatorname{tr}_{+}\left(\not p_{i} \not p_{j} \not p_{k} \not p_{l}\right)=\frac{1}{2} \operatorname{tr}\left(\left(1-\gamma_{5}\right) p_{i} p_{j} p_{k} \not p_{l}\right)=\langle i j\rangle[j k]\langle k l\rangle[l i], \\
& \operatorname{tr}_{-}\left(p_{i} p_{j} p_{k} p_{l}\right)=\frac{1}{2} \operatorname{tr}\left(\left(1+\gamma_{5}\right) p_{i} p_{j} p_{k} p_{l}\right)=[i j]\langle j k\rangle[k l]\langle l i\rangle .
\end{aligned}
$$

We can build up arbitrarily large spinor products using,

$$
\langle i j\rangle[j k]=\langle i|j| k]
$$

The spinors also satisfy a Schouten identity,

$$
\langle i j\rangle\langle k l\rangle=\langle i k\rangle\langle j l\rangle+\langle i l\rangle\langle k j\rangle \text {. }
$$

Massless spin 1 particles have two transverse polarisations/helicities. Cru-

cially the two physical polarisation vectors can also be written in the spinor helicity formalism,

$$
\epsilon_{\mu}^{-}(p, q)=\frac{\left[q\left|\gamma_{\mu}\right| p\right\rangle}{\sqrt{2}[p q]}, \quad \epsilon_{\mu}^{+}(p, q)=\frac{\left[p\left|\gamma_{\mu}\right| q\right\rangle}{\sqrt{2}\langle q p\rangle},
$$

where,

$$
\epsilon_{\mu}^{+} \epsilon^{\mu+}=0, \quad \epsilon_{\mu}^{-} \epsilon^{\mu-}=0 .
$$




\subsection{Spinor Helicity Formalism}

The polarisation vectors satisfy the completeness relation,

$$
\sum_{h= \pm} \epsilon_{\mu}^{h}(p, q) \epsilon_{\nu}^{h}(p, q)^{*}=-\eta_{\mu \nu}+2 \frac{p_{\mu} q_{\nu}+q_{\mu} p_{\nu}}{2 q \cdot p}
$$

related to the propagator of the gluon in the lightcone gauge. In fact, we use this relation when a propagator goes on shell and an amplitude factorises into the product of two lower multiplicity amplitudes and a propagator. There is an implicit sum over the possible helicity assignments of the on-shell internal leg arising from (2.68).

Additionally, following work in this gauge [122, 132] we are able to continue momenta off-shell with the addition of an non-physical polarisation vector that is absorbed into a redefinition of the four-point vertex ${ }^{1}$. The off-shell continuation can be written as

$$
p^{\mu, b}=p^{\mu}-\frac{p^{2}}{2 p \cdot q} q^{\mu}
$$

where $p^{\mu, b}$ denotes an on-shell momentum and the unflattened momentum, $p^{\mu}$, is off-shell $p^{2} \neq 0$. If $p^{2}=0$, then we have $p^{b, \mu}=p^{\mu}$.

This can be implemented explicitly, or by ensuring that the external momentum we wish to continue off-shell are contracted with the axial gauge reference momentum $q$.

Massless gravitons are also considered in this thesis. These massless spin 2 particles have two polarisations and can be constructed using the Yang-Mills gluon polarisations via,

$$
\epsilon_{\mu \nu}^{++}(p, q)=\epsilon_{\mu}^{+}(p, q) \epsilon_{\nu}^{+}(p, q) \quad \epsilon_{\mu \nu}^{--}(p, q)=\epsilon_{\mu}^{-}(p, q) \epsilon_{\nu}^{-}(p, q) .
$$

\footnotetext{
${ }^{1}$ We are also free to choose a different lightcone gauge for each external leg in a partial amplitude - corresponding to a choice of the reference spinor $q_{i}$ in the polarisation vectors - in order to simplify a calculation.
} 


\section{REVIEW OF TECHNIQUES FOR CALCULATING SCATTERING AMPLITUDES}

\subsubsection{Spinor Weight: Little Group Scaling}

We have seen that the Weyl spinors have a scaling freedom afforded to them from the definition of the four momentum as a bispinor,

$$
\begin{gathered}
p_{i}^{\alpha \dot{\beta}}=\lambda_{i}^{\alpha} \bar{\lambda}_{i}^{\dot{\beta}}, \\
\lambda_{i}^{\alpha}=|i\rangle \rightarrow t|i\rangle, \quad \bar{\lambda}_{i}^{\dot{\beta}}=\left[i \mid \rightarrow t^{-1}[i \mid .\right.
\end{gathered}
$$

Glimpsing at the Feynman rules when computing a partial amplitude for a moment, we see we must dot polarisation vectors with the Feynman rules for an interaction vertex. The interaction vertex only depends on the momentum, therefore the polarisation vectors are the only source of the little group scale dependence in the partial amplitudes. The helicity of the external gluons dictates this scale dependence. Under the scaling (2.72), the polarisation vectors scale as

$$
\begin{aligned}
\epsilon_{\mu}^{-}(p, q) & =\frac{\left[q\left|\gamma_{\mu}\right| p\right\rangle}{\sqrt{2}[p q]} \sim t^{2}, \\
\epsilon_{\mu}^{+}(p, q) & =\frac{\left[p\left|\gamma_{\mu}\right| q\right\rangle}{\sqrt{2}\langle q p\rangle} \sim t^{-2} .
\end{aligned}
$$

implying a partial amplitude scales as

$$
A\left(\ldots, i^{h_{i}}, \ldots\right) \sim t^{-2 h_{i}},
$$

for each on-shell gluon. We use the convention that the $\lambda$ 's have spinor weight +1 and the $\bar{\lambda}$ 's have spinor weight -1 . These constraints, along with the mass dimension of an interaction (and the symmetry of the partial amplitude in some rational one-loop amplitudes [89]) is enough to fully determine the form of the three-point vertices of a theory and some rational one-loop amplitudes. For example, we can circumvent the use of the Feynman rules directly to write down the unique form of the three-gluon MHV complex amplitudes. 


\subsection{From Feynman Diagrams to On-shell Am- plitudes}

In this short section, we take a look at two methods to obtain the on-shell threepoint amplitudes that are subsequently used in recursive techniques to compute higher multiplicity amplitudes.

We can construct a tree-level three-point MHV complex amplitude by considering the Feynman rules for the three-point interactions. This amplitude is vanishing in real momenta, but by considering the insight of Witten [27] we can construct a non-vanishing amplitude by writing $p_{i}=\lambda_{i} \bar{\lambda}_{i}$ with $p_{i} \in \mathbb{C}$.

The colour ordered Feynman rule for the three-gluon interaction vertex is given by,

$$
V_{3}\left(p_{1}^{\mu}, p_{2}^{\nu}, p_{3}^{\rho}\right)=\left[g^{\mu \nu}\left(p_{1}-p_{2}\right)^{\rho}+g^{\nu \rho}\left(p_{2}-p_{3}\right)^{\mu}+g^{\rho \mu}\left(p_{3}-p_{1}\right)^{\nu}\right],
$$

wherel we contract the vertex with the appropriate polarisation vectors for each external gluon,

$$
\epsilon_{\mu}^{-}\left(p_{i}, q_{i}\right)=\frac{\left[q_{i}\left|\gamma_{\mu}\right| p_{i}\right\rangle}{\sqrt{2}\left[p_{i} q_{i}\right]}, \quad \epsilon_{\mu}^{+}\left(p_{i}, q_{i}\right)=\frac{\left[p_{i}\left|\gamma_{\mu}\right| q_{i}\right\rangle}{\sqrt{2}\left\langle q_{i} p_{i}\right\rangle} .
$$

Applying these rules, the MHV three-point amplitude is written in terms of a reference spinor? 2 ,

$$
A_{3}^{(0)}\left(1^{-}, 2^{-}, 3^{+}\right)=\epsilon_{\mu}^{-}\left(p_{1}, q_{1}\right) \epsilon_{\nu}^{-}\left(p_{2}, q_{2}\right) \epsilon_{\rho}^{+}\left(p_{3}, q_{3}\right) V_{3}\left(p_{1}^{\mu}, p_{2}^{\nu}, p_{3}^{\rho}\right) \propto \frac{\langle 12\rangle[3 q]^{2}}{[1 q][2 q]} .
$$

Including the axial gauge reference spinor in this way allows each of the three gluons to be trivially continued off-shell through (2.69).

Alternatively, through a line of additional manipulation, we may write the

\footnotetext{
${ }^{1}$ We have also removed a factor of $i$ from the definition due to (2.17).

${ }^{2}$ Here, we set $q_{1}=q_{2}=q_{3}=q$.
} 


\section{REVIEW OF TECHNIQUES FOR CALCULATING SCATTERING AMPLITUDES}

amplitude in a form where the gluons are explicitly on-shell,

$$
A_{3}^{(0)}\left(1^{-}, 2^{-}, 3^{+}\right)=\frac{\langle 12\rangle^{3}}{\langle 23\rangle\langle 31\rangle} .
$$

This is a special case of the Parke-Taylor formula [19] for the $n$-gluon MHV amplitude

$$
A_{n}^{(0)}\left(1^{+}, \ldots, i^{-}, \ldots, j^{-}, \ldots, n^{+}\right)=\frac{\langle i j\rangle^{4}}{\langle 12\rangle \cdots\langle n 1\rangle} .
$$

The three-point MHV amplitude we have looked at is a simple example for which we have only needed to consider one diagram and we have been able to keep the gauge general, i.e. we did not fix the value of $q$, the reference momentum. As a side note; the four-gluon MHV amplitude involves the calculation of three Feynman diagrams, the s-channel, the t-channel and the four-point interaction vertex - each of which is not a gauge invariant quantity by itself. In calculating the four-point amplitude we can associate the axial gauge reference spinors to the momenta of the external gluons to give a vanishing contribution from the contact term.

Alternatively, we can approach the construction of these partial amplitudes from a related but simpler method. The form of the three-point MHV complex amplitude can be constrained using two principles; locality and Lorentz invariance. Schematically, the three-point gauge boson interaction term in the Yang-Mills Lagrangian is given by $A A \partial A$. Fourier transforming to momentum space, where amplitudes are most naturally written, tells us that this interaction has mass dimension (or momentum weight) one. This is confirmed by checking the Feynman rule for a three-point interaction. Locality, determines the momentum weight of the interaction then. Furthermore, we know how the amplitude will scale under the Little group (its spinor weight) given the helicity assignment of the external legs. These two conditions uniquely determine the form of the on-shell amplitude to be (2.79).

For the three-point MHV gluon amplitude, $A_{3}^{(0)}\left(1^{-}, 2^{-}, 3^{+}\right)$, we know the amplitude must have spinor weight 1 in the legs $p_{1}$ and $p_{2}$ and spinor weight -1 in 
leg $p_{3}$. Additionally, since three-point kinematics implies

$$
\begin{aligned}
& 2 p_{i} \cdot p_{j}=0, \quad i<j=1,2,3, \\
& \langle i j\rangle[j i]=0 .
\end{aligned}
$$

and for $p \in \mathbb{C}$, we are free to choose either $\langle i j\rangle=0$ with $[j i]=0$ or vice versa. The first condition is the only choice that satisfies the spinor weight conditions in a non-trivial way. The momentum weight of the spinor brackets can be determined from the relation $2 p_{1} \cdot p_{j}=\langle i j\rangle[j i]$ which has momentum weight 2 , therefore each spinor bracket has momentum weight 1 . Resulting in,

$$
A_{3}^{(0)}\left(1^{-}, 2^{-}, 3^{+}\right)=\frac{\langle 12\rangle^{3}}{\langle 23\rangle\langle 31\rangle},
$$

which has momentum weight 1 and the correct spinor weight for the external legs.

The three-point Einstein-Gravity amplitudes can be fixed in this way, too. In chapter 5, we go further and look at how we may define the tree-level $S$-matrix of an extended theory of gravity from exactly these principles.

We cannot fix the form of the four-point amplitude in this way, but instead determine its form through BCFW recursion [35, 36] which utilises complex recursion and factorisation theorems. In order to understand this methodology we subsequently review the factorisation theorems of tree and loop level amplitudes.

\subsection{Tree-Level Amplitudes: Analytic Structure and Factorisation}

Amplitudes exhibit many different properties as we have already seen, however they also possess additional features owing to their analytic structure. For treelevel amplitudes, singularities arise from internal propagators connecting sums of external momenta in the limit that the propagator momenta goes to zero. These limits, apart from showing the interesting behaviour of amplitudes in particular regions of momentum space, are strong consistency checks on a calculation and 


\section{REVIEW OF TECHNIQUES FOR CALCULATING SCATTERING AMPLITUDES}

furthermore place strong constraints on the form of an amplitude. The singularities in these limits for tree-level amplitudes are simple poles in the spinor brackets. In the limit that the propagator goes on-shell, the amplitude factorises into the product of the two on-shell amplitudes partitioned by this propagator. Multiparticle factorisations occur when there is a sum of three or more external legs on either side of the propagator 1 ,

$A_{n}^{(0)}(1, \ldots, i, i+1, \ldots, n) \underset{K^{2} \rightarrow 0}{\longrightarrow} \sum_{h= \pm} A_{i+1}^{(0)}\left(1, \ldots, i, K^{h}\right) \frac{-1}{K^{2}} A_{n-i+1}^{(0)}\left(-K^{-h}, i+1, \ldots, n\right)$

where $K=p_{1 \ldots i}=p_{1}+\ldots+p_{i}$ and we have defined the legs in a cyclic order here, but is applicable to any partitioned set of legs. Note that the gluon propagator has been replaced by $-1 / k^{2}$ multiplied by a sum over polarisation vectors (2.68).

In the special case of $i=2$, we have a collinear singularity. In this limit, the two external gluons are close to collinear since $2 p_{1} \cdot p_{2} \rightarrow 0$ and the amplitude exhibits a universal behaviour,

$A_{n}^{(0)}\left(1^{h_{1}}, \ldots, i^{h_{i}}, j^{h_{j}}, \ldots, n^{h_{n}}\right) \underset{i \| j}{\longrightarrow} \sum_{h= \pm} \operatorname{Split}_{h}^{(0)}\left(p_{i}^{h_{i}}, p_{j}^{h_{j}}, z\right) A_{n-1}^{(0)}\left(1^{h_{1}}, \ldots, k^{-h}, \ldots, n^{h_{n}}\right)$

The universal behaviour characterised by the splitting functions can be investigated by parametrising the collinear limit as

$$
p_{i}=(1-z) k, \quad p_{j}=z k,
$$

\footnotetext{
${ }^{1}$ Ordinarily the propagator is multiplied by a factor of $i$, but since we have defined the amplitudes factoring out $i$, the equivalent formalism in the BCFW relations is to include a factor of -1 .
} 


\subsection{Tree-Level Amplitudes: Analytic Structure and Factorisation}

where $k=p_{i}+p_{j}$. The tree-level splitting functions are given by [19, 133, 134],

$$
\begin{aligned}
\operatorname{Split}_{+}^{(0)}\left(i^{+}, j^{-}\right) & =\frac{1}{\langle a b\rangle} \frac{(1-z)^{2}}{\sqrt{z(1-z)}}, \\
\operatorname{Split}_{-}^{(0)}\left(i^{+}, j^{-}\right) & =\frac{1}{[b a]} \frac{z^{2}}{\sqrt{z(1-z)}}, \\
\operatorname{Split}_{-}^{(0)}\left(i^{+}, j^{+}\right) & =\frac{1}{\langle a b\rangle} \frac{1}{\sqrt{z(1-z)}}, \\
\operatorname{Split}_{-}^{(0)}\left(i^{-}, j^{-}\right) & =0 .
\end{aligned}
$$

The splitting functions depend on the helicities of the collinear legs $p_{i}$ and $p_{j}$, the helicity of the intermediate particle $k$ and the collinear parameter $z$. The remaining splitting functions can be related to these by conjugation.

Tree-level amplitudes also have universal factorisation behaviour when the momenta of an external particle goes to zero, i.e. a soft limit [133, 134].

In Yang-Mills, the soft limit is described by,

$$
A_{n}^{(0)}(1, \ldots, i, s, j, \ldots, n) \underset{p_{s} \rightarrow 0}{\longrightarrow} \operatorname{Soft}^{(0)}(i, s, j) A_{n-1}^{(0)}(1, \ldots, i, j, \ldots, n)
$$

where the tree-level soft factor is independent of the helicities of the soft leg and its adjacent legs

$$
\operatorname{Soft}^{(0)}(i, s, j)=\frac{\langle i j\rangle}{\langle i s\rangle\langle s j\rangle}
$$

Gravity amplitudes also have soft limits 114 117 that are exposed in the variable $t$ as $t \rightarrow 0$, when a positive helicity leg is parametrised as $p_{i}=t p_{s}$. When using complex momenta, gravity amplitudes have soft limits to sub-subleading order in $t$. This is examined in the context of a calculation in an extended theory of gravity in section 5.3.

Understanding the factorisation properties has proved to be a crucial step in simplifying calculations in the study of Yang-Mills and Gravity amplitudes. In the following section we review techniques that rely on these properties to construct rational amplitudes: complex recursion and the BCFW formalism. 


\section{REVIEW OF TECHNIQUES FOR CALCULATING SCATTERING AMPLITUDES}

\subsection{Complex Recursion}

Complex recursion is a term describing techniques that are used to determine rational amplitudes, or rational parts of amplitudes by use of complex analysis and factorisation theorems. In general, shifting the external momenta of an amplitude by a complex momentum $z$, whilst maintaining overall conservation of momentum, transforms the amplitude into a meromorphic function in the variable $z$. The poles in the complex variable $z$ correspond to regions of momentum space for which an intermediate gluon i.e. a propagator, goes on-shell. For tree amplitudes only simple poles are present but for loop amplitudes and in particular the one- and two-loop amplitudes discussed in this thesis the highest order poles are double poles. The residues of the highest order terms in the Laurent series around such poles coincide with factorisations of the amplitude on collinear and multiparticle poles. This allows the amplitude to be expressed as a sum of products of two lower-point on-shell gauge invariant amplitudes and the propagator separating them.

This was a powerful observation of Britto, Cachazo, Feng and Witten [36] who employed a very simple shift of the external momenta to prove that the recurrence relation [35] detailed below can be used to express tree-level amplitudes as a product of complex three-point amplitudes [27] and propagators. This technique has also been implemented with the use of other shifts, namely the all-line shift 135] and Risager shift [37].

\subsubsection{BCFW Recursion}

Tree-level amplitudes have the simplest analytic structure of all the amplitudes present in a pertubative expansion of the gauge coupling, $\alpha$. They are rational functions of the external momenta and locality imposes the condition that interactions occur at the same point in spacetime and are mediated by the transfer of momentum by an intermediate particle. This implies that tree-level amplitudes only contain simple poles that correspond to regions of momentum space where internal propagators go on-shell. We can exploit this analytic structure using complex momentum to build $n$-point tree-level amplitudes from the product of 
three-point tree-level amplitudes and a propagator connecting them. Defining a complex shift that acts on two external momenta $p_{i}$ and $p_{j}$,

$$
\begin{aligned}
& \bar{\lambda}_{i} \rightarrow \hat{\bar{\lambda}}_{i}=\bar{\lambda}_{i}-z \bar{\lambda}_{j}, \\
& \lambda_{j} \rightarrow \hat{\lambda}_{j}=\lambda_{j}+z \lambda_{i},
\end{aligned}
$$

where $\hat{p}_{i}$ and $\hat{p}_{j}$ are on either side of the propagator and become

$$
\hat{p}_{i}=p_{i}(z)=p_{i}-z \omega \quad \hat{p}_{j}=p_{j}(z)=p_{j}+z \omega
$$

with $\omega=\lambda_{j} \bar{\lambda}_{i}$. The shift is designed such that conservation of momentum is preserved and all external lines remain null,

$$
\begin{aligned}
p_{i}(z)+p_{j}(z) & =p_{i}-z \omega+p_{j}+z \omega=p_{i}+p_{j}, \\
p_{i}^{2}(z) & =p_{j}^{2}(z)=0
\end{aligned}
$$

where the following arise from the definition of $\omega$,

$$
\omega^{2}=0, \quad 2 \omega \cdot p_{i}=2 \omega \cdot p_{j}=0
$$

such that the shift passes a $z$-dependence to the propagator separating the two legs.

The discussion that follows will be limited to tree-level amplitudes due to the complication that double poles present in loop amplitudes. The use of complex recursion with regards to loop amplitudes containing double poles requires an extension that we dedicate to a later section.

Take for example a tree-level $n$-point partial amplitude

$$
\operatorname{Tr}\left(T^{a_{1}} T^{a_{2}} \cdots T^{a_{n}}\right) A_{n}^{(0)}\left(p_{1}, p_{2}, \cdots, p_{n}\right),
$$

where the momenta have a cyclic ordering and obey conservation of momentum,

$$
p_{1}+p_{2}+\cdots+p_{n}=0 .
$$

With $\hat{p}_{i}$ and $\hat{p}_{j}$ on either side of a propagator, the shifted propagator can be 


\section{REVIEW OF TECHNIQUES FOR CALCULATING SCATTERING AMPLITUDES}

written as,

$$
\begin{gathered}
\hat{p}_{1 \cdots i} \equiv \hat{p}_{1 \cdots i}(z)=p_{1}+\cdots+\hat{p}_{i} \\
\hat{p}_{1 \cdots i}^{2} \equiv \hat{p}_{1 \cdots i}^{2}(z)=p_{1 \cdots i}^{2}-z\left\langle j\left|p_{1 \cdots i}\right| i\right]=-\frac{z-z_{I}}{z_{I}} p_{1 \cdots i}^{2},
\end{gathered}
$$

where $z_{I}$ is the location of the pole in the complex plane.

It is clear from the second line of eq. (2.101) that the shifted propagator $p_{1 \cdots i}^{2}(z)$ contributes a simple pole at $z=z_{I}$ to the amplitude. This meromorphic function of $z$ will contain simple poles at various points in the complex plane corresponding to different internal propagators going on shell. It follows then that an amplitude contains a pole for every allowed group of momenta going on shell ${ }^{1}$, and by identifying the poles and their corresponding residues we can uniquely determine the form of the tree-level amplitude.

Consider the contour integral,

$$
\frac{1}{2 \pi i} \oint_{\gamma} \frac{A^{(0)}(z)}{z} \mathrm{~d} z
$$

where $\gamma$ is a circular contour taken to infinity, encompassing all the poles of the amplitude and the pole at $z=0$ that we have inserted. The pole corresponding to $z=0$ is not a propagator pole, rather it's a pole whose residue returns the amplitude we are interested in calculating, $A^{(0)}(0)$.

The remaining poles in $A^{(0)}(z)$ are simple poles in $z$ belonging to propagator poles. By use of Cauchy's residue theorem we can write

$$
\frac{1}{2 \pi i} \oint_{\gamma} d z \frac{A_{n}^{(0)}(z)}{z}=A_{n}^{(0)}(0)+\sum_{z_{I} \neq 0} \operatorname{Res}\left[\frac{A_{n}^{(0)}(z)}{z}, z_{I}\right]
$$

and if the amplitude vanishes at infinity, $A(z) \rightarrow 0$ as $z \rightarrow 0$, the contour integral

\footnotetext{
${ }^{1} \mathrm{By}$ allowed group, we mean configurations of the external momenta allowed after colour decomposition. For a partial amplitude multiplied by $\operatorname{Tr}\left(T^{a_{1}} T^{a_{2}} \cdots T^{a_{n}}\right)$, this would be only cyclically ordered momenta.
} 
vanishes. The amplitude is given as a sum of residues of the propagator poles,

$$
A_{n}^{(0)}(0)=-\sum_{z_{I}} \operatorname{Res}\left[\frac{A_{n}^{(0)}(z)}{z}, z_{I}\right] .
$$

This is a statement of complex analysis of which we would like to connect to physics. We have seen that on collinear and multiparticle poles, tree-level amplitudes factorise

$A_{n}^{(0)}(1,2, \cdots, n) \underset{p_{1 \ldots i}^{2} \rightarrow 0}{\longrightarrow} \sum_{h= \pm} A_{i+1}^{(0)}\left(1, \cdots, i, K^{h}\right) \frac{-1}{K^{2}} A_{n-i+1}^{(0)}\left(-K^{-h},(i+1), \cdots, n\right)$

where we must sum over all helicity configurations of the internal propagator that goes on shell. Both lower multiplicity amplitudes are on-shell amplitudes, allowing for a recursive implementation.

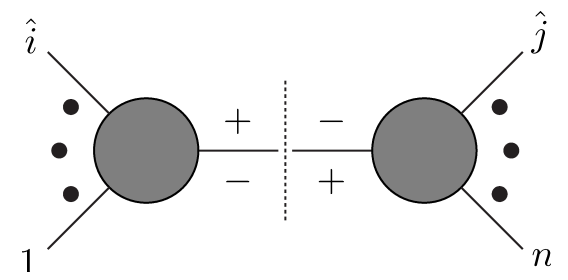

Figure 2.5: Diagrammatic representation of an amplitude factorising as the internal propagator $t_{1 \ldots n}$, goes on-shell. The factorisation includes a sum over internal helicity configurations.

The key to this technique is calculating the residues. As the residues are the amplitude evaluated on the simple propagator poles, we may use the factorisation property in the calculation of the residues. Without loss of generality, we define the legs to be in a cyclic order with $j=i+1$ and define the shifted legs to be $\hat{p}_{i}$ 


\section{REVIEW OF TECHNIQUES FOR CALCULATING SCATTERING AMPLITUDES}

and $\hat{p}_{j}$. The residues are given as,

$$
\begin{aligned}
\left.\operatorname{Res}\left[\frac{\hat{A}_{n}^{(0)}(z)}{z}\right]\right|_{z_{I}} & =\operatorname{Res}\left[\sum_{h= \pm} \frac{A_{i+1}^{(0)}\left(p_{1}, \ldots, \hat{p}_{i}, \hat{K}^{h} ; z\right) A_{n-i+1}^{(0)}\left(-\hat{K}^{-h}, \hat{p}_{j}, \ldots p_{n} ; z\right)}{\hat{p}_{1 \ldots i}^{2}(z)} \frac{1}{z}, z_{I}\right] \\
& =\lim _{z \rightarrow z_{I}}\left(\frac{\left(z-z_{I}\right)}{z} \sum_{h= \pm} \frac{A_{i+1}^{(0)}\left(p_{1}, \ldots, \hat{p}_{i}, \hat{K}^{h} ; z\right) A_{n-i+1}^{(0)}\left(-\hat{K}^{-h}, \hat{p}_{j}, \ldots p_{n} ; z\right)}{\hat{p}_{1 \cdots i}^{2}(z)}\right)
\end{aligned}
$$

Rewriting the propagator using eq.(2.101) cancels the pole in the propagator located at $z_{i}$ and the resultant expression is the original $z$-independent propagator multiplied by the factorised sub-amplitudes evaluated at $z=z_{I}$,

$$
\left.\operatorname{Res}\left[\frac{\hat{A}_{n}^{(0)}(z)}{z}\right]\right|_{z_{I}}=\sum_{h= \pm} \frac{A_{i+1}^{(0)}\left(p_{1}, \ldots, \hat{p}_{i}, \hat{K}^{h} ; z_{I}\right) A_{n-i+1}^{(0)}\left(-\hat{K}^{-h}, \hat{p}_{j}, \ldots p_{n} ; z_{I}\right)}{p_{1 \ldots i}^{2}}
$$

Then providing the contour integral vanishes, we may calculate a tree amplitude via the following relation,

$$
A_{n}^{(0)}(0)=-\sum_{z_{i}} \sum_{h= \pm} \frac{A_{i+1}^{(0)}\left(p_{1}, \ldots, \hat{p}_{i}, \hat{K}^{h} ; z_{i}\right) A_{n-i+1}^{(0)}\left(-\hat{K}^{-h}, \hat{p}_{j}, \ldots p_{n} ; z_{i}\right)}{p_{1 \cdots i}^{2}} .
$$

The sum over $z_{i}$ implies that all independent configurations of the external legs that admit a different shifted propagator must be summed over after taking their residues.

The property that the shifted amplitude vanishes at infinity is a necessary condition for the recursion to work. This has been investigated using a background field method 136 to determine the large $|z|$ behaviour of tree-level amplitudes under various shifts. The proof covers amplitudes in gauge theories and general relativity and shows this behaviour up to $n=10$ for all helicity configurations in gravity amplitudes [137]. Clearly, there are four combinations of the types of legs that can be shifted given gluons and massless gravitons have two helicities. The behaviour of the amplitude under the BCFW shifts are summarised 


\begin{tabular}{|c|c|c|c|c|}
\hline$[i, j\rangle$ & {$[-,-\rangle$} & {$[-,+\rangle$} & {$[+,-\rangle$} & {$[+,+\rangle$} \\
\hline$A(z)$ & $z^{-1}$ & $z^{-1}$ & $z^{3}$ & $z^{-1}$ \\
\hline$M(z)$ & $z^{-2}$ & $z^{-2}$ & $z^{6}$ & $z^{-2}$ \\
\hline
\end{tabular}

Table 2.1: The large $|z|$ behaviour of gauge and gravity amplitudes under BCFW shifts.

in table. 2.1. For the rational part of loop amplitudes the large $|z|$ behaviour is hard to tell a priori, however we can get an indication via less elegant means. The complex shifts break the symmetry of a partial amplitude by deforming the momenta of some specified legs position in the complex plane. If this symmetry is not recovered after performing the complex recursion, this is an indication that the shifted amplitude does not vanish at infinity. The fact that one recovers the symmetry of the amplitude then, is a strong check that the amplitude has been calculated correctly. In the cases where a BCFW shift does not produce the desired behaviour at infinity we can attempt to employ a different shift. One such case is the rational part of the two-loop all-plus amplitude, where we employ the Risager shift.

The Risager shift [37] originally employed to prove the CSW rules [29] deforms three momenta, say $p_{i}, p_{j}$ and $p_{k}$, by

$$
\begin{aligned}
\lambda_{i} & \rightarrow \hat{\lambda}_{i}=\lambda_{i}+z[j k] \lambda_{\eta}, \\
\lambda_{j} \rightarrow \hat{\lambda}_{j} & =\lambda_{j}+z[k i] \lambda_{\eta}, \\
\lambda_{k} \rightarrow \hat{\lambda}_{k} & =\lambda_{k}+z[i j] \lambda_{\eta},
\end{aligned}
$$

where $\lambda_{\eta}$ must satisfy $\langle i \eta\rangle \neq 0,\langle j \eta\rangle \neq 0$ and $\langle k \eta\rangle \neq 0$ but is otherwise unconstrained. Under the Risager shift, the two-loop all-plus amplitude does have the desired asymptotic behaviour and so when we come to calculate the rational part of the two-loop all-plus we will use this shift.

Complex recursion can therefore determine any amplitude that contains only single poles. In chapter 5 we briefly discuss the application of complex recursion to Einstein-Hilbert gravity and use these recursive techniques to determine the tree-level $S$-matrix elements for an extended theory of gravity. Having shown a 


\section{REVIEW OF TECHNIQUES FOR CALCULATING SCATTERING AMPLITUDES}

method of completely determining the tree-level amplitudes we move to discussing amplitudes that appear at higher orders in perturbation theory. In general these amplitudes have a far more complicated singularity structure, owing to the integrations that must be performed over the loop momenta. In the next section we discuss the intricacies associated to the loop integrations and explore the various techniques used to simplify and efficiently compute one-loop amplitudes and a special two-loop amplitude, the all-plus. 


\subsection{Loop-Amplitudes}

Higher orders in a perturbative treatment of a quantum field theory are due to virtual corrections to the tree-level amplitudes. These corrections amount to amplitudes that contain loops of intermediate virtual particles whose momenta can range from $-\infty \leq \ell \leq+\infty$. This extra degree of freedom must be integrated over this range. For each higher order in the pertubative expansion of the coupling constant, each amplitude gains an additional virtual loop,

$$
\mathcal{A}_{n}=\delta^{(4)}\left(\sum_{i}^{n} p_{i}\right) i g^{n-2} \sum_{L \geq 0} a^{L} \mathcal{A}_{n}^{(L)} .
$$

In this section we limit the discussion of loop amplitudes mainly to general one-loop amplitudes and a particular helicity configuration of two-loop amplitudes, the all-plus. We review dimensional regularisation [48], a method of taming the ultraviolet (UV) and infrared (IR) infinities [32, 33] that arise from high and low energy regions of the loop integration respectively. Loop integrals of general $n$-point amplitudes can have up to $n$ propagators and $n$ powers of the loop momentum 1 . We outline integral reduction methods [52] at one-loop that express the amplitude in terms of a simpler basis of integrals with a rational ambiguity [49 51, 54, 55, 138, 139]. We show how we utilise generalised four-dimensional unitarity [30, 46, 47, 53, 56] to construct the amplitude from its branch cut singularity structure. The last section is dedicated to a specific calculational tool augmented recursion - developed in refs. [81, 84 89. to deduce the rational parts of one- and two-loop amplitudes in Yang-Mills and Gravity. This technique is used in chapter 3 and chapter 4 to generate the rational parts of a leading in colour six- and seven-gluon amplitude and a full colour five-gluon amplitude.

\footnotetext{
${ }^{1}$ This is in Yang-Mills. In gravity there may be up to $2 n$ powers in the numerator.

${ }^{2}$ These integrals evaluate to functions that contain branch cut singularities.
} 


\section{REVIEW OF TECHNIQUES FOR CALCULATING SCATTERING AMPLITUDES}

\subsubsection{Dimensional Regularisation}

The Feynman integrals that arise in loop computations diverge in four dimensions. The bubble integral,

$$
I_{2}^{D=4}=\int \frac{d^{4} \ell}{(2 \pi)^{4}} \frac{1}{\ell^{2}\left(\ell-p_{i j}\right)^{2}}
$$

has a logarithmic divergence in the ultraviolet as $\ell \rightarrow \infty$, but is finite in the IR as $\ell \rightarrow 0$. However, the one-mass triangle integral 1 has IR divergences,

$$
I_{3}^{1 m, D=4}=\int \frac{d^{4} \ell}{(2 \pi)^{4}} \frac{1}{\ell^{2}\left(\ell-p_{i j}\right)^{2}\left(\ell+p_{n}\right)^{2}},
$$

where $p_{i j}^{2} \neq 0$ but $p_{n}^{2}=0$.

In order to regulate these divergences, we analytically continue away from $D=4$ dimensions to $D=4-2 \epsilon$ dimensions using dimensional regularisation [48]. In this scheme, we choose the external particles to be described in four dimensions, whereas the loop momenta are in $D=4-2 \epsilon$. The main advantage of using dimensional regularisation is that it regularises both UV and IR divergences simultaneously, such that calculations of unrenormalised amplitudes can be performed.

Dimensional regularisation has important ramifications for the theory in question. Firstly, massless tadpole integrals that could appear in pure Yang-Mills vanish due to the lack of a mass scale in the integral.

$$
\int \frac{d^{D} \ell}{(2 \pi)^{D}} \frac{1}{\ell^{2}}=0
$$

This is the reason that the integral basis for one-loop amplitudes shown in subsection 2.8.2 contains integrals with at least two propagators.

Secondly, it allows for an organisation of the amplitude in terms of its singularity structure in $\epsilon$. For the two-loop all-plus unrenormalised amplitude, the UV and collinear IR singularities cancel leaving only the soft IR singularities that are $\mathcal{O}\left(\epsilon^{-2}\right)[32,33,96]$. This particular amplitude can then be organised into its soft

\footnotetext{
${ }^{1}$ amongst other types of integrals that can appear
} 
IR contribution, plus other finite terms. We show this organisation in chapter 3 . Finally, the IR and UV divergences of the integrals, and by proxy the amplitude as a whole, are shifted to singularities in the dimensional regularisation parameter $\epsilon$ as we take the limit $\epsilon \rightarrow 0$. Although this procedure mixes these singularities ${ }^{1}$ it makes the singularity structure manifest in $\epsilon$. At the heart of this discussion on the singularities in the loop integration is the need to control the numerical instabilities these divergences present in cross-section calculations. Understanding the nature of the UV and IR singularities facilitates a relatively straightforward renormalisation of the amplitude using, for examples the $\overline{M S}$ scheme. Additionally, the IR singularities can be cancelled in the full crosssection as a result of the KLN theorem.

Grasping the nature of the divergent integrals is then of paramount importance for subsequent phenomenological calculations.

\subsubsection{Integral Reduction of One-Loop Amplitudes}

A multitude of Feynman integrals appear when considering the amplitudes in a pertubative expansion of a quantum field theory. At one-loop for an $n$-point pure Yang-Mills amplitude, it is possible in general to encounter Feynman integrals with $n$-propagators and polynomials in the numerator consisting of up to $n$ powers of the loop momental,

$$
I_{n}=\int \frac{d^{D} \ell}{(2 \pi)^{D}} \frac{\mathcal{P}(\ell)}{\ell^{2}\left(\ell-p_{1}\right)^{2} \cdots\left(\ell+p_{n}\right)^{2}},
$$

where $\mathcal{P}(\ell)$ is some general polynomial in the loop momentum.

It is possible, however, to reduce a general $n$-point integral to a sum of box, triangle and bubble integrals in $D=4$ [49 51]. This can be achieved using Passarino-Veltman reduction [52]. Given the loop momenta and external momenta are linearly dependent, the loop dependent numerator can be written in a basis of external momenta ${ }^{3}$. This sets up a system of equations for which

\footnotetext{
${ }^{1}$ For example in a one-loop amplitude simple poles in $\epsilon$ correspond to both UV and IR divergences.

${ }^{2}$ In gravity amplitudes there can be up to $2 n$ powers of the loop momenta.

3 and combinations of the metric tensor for even powers of the loop momenta.
} 


\section{REVIEW OF TECHNIQUES FOR CALCULATING SCATTERING AMPLITUDES}

each term in the basis can be used to cancel propagators in the original integral provided the external momenta are in $D=4$-dimensions.

Repeated application of this procedure reduces tensor integrals with $n$-propagators to scalar integrals with up to four propagators [138, 139]. The reduction coefficients found by this approach are restricted to four dimensions and thus this approach - which does not take into account the $-2 \epsilon$ additional dimensions of the loop momenta in the numerator - leaves a rational ambiguity at $\mathcal{O}\left(\epsilon^{0}\right)$. An example that illustrates this discrepancy comes from refs. [49, 50] who showed that the $D=4$ dimensional pentagon integral can be reduced to a sum of five boxes. However, an alternative dimensionally regulated approach [54, 55] using a Feynman parameter representation showed this is true with the addition of the scalar pentagon in $D=6-2 \epsilon$ dimensions with an $\mathcal{O}(\epsilon)$ coefficient. Keeping track of the orthogonal $-2 \epsilon$ dimensions in the reduction leads to integrals that depend on even powers of this orthogonal loop momentum component, $\left(\mu^{2}\right)$, that ultimately integrate to rational contributions. This is discussed briefly in subsection 2.9.3. Either approach, once taking the $D=4$ limit for external particles and ignoring contributions of $\mathcal{O}(\epsilon)$ and above, leads to a description of a one-loop amplitude in terms of a basis of integrals,

$$
A_{n}^{(1)}=\sum_{i} c_{4, i} I_{4, i}+\sum_{i} c_{3, i} I_{3, i}+\sum_{i} c_{2, i} I_{2, i}+R_{n}
$$

where the coefficients are rational functions of the external momenta in $D=4$ dimensions. The sum is over all possible configurations of scalar box, triangle and bubble integral functions where for example four-,three-,two- and one-mass box integrals can appear. The integrals are summarised in Appendix B.

Given this integral basis, we can better understand the UV and IR singularity structure of one-loop amplitudes. 


\subsection{Loop-Amplitudes}

\subsubsection{UV and IR singularities}

We work with unrenormalised amplitudes, where the UV divergences are poles in the dimensional regulator. Given the one-loop integral basis, it is possible to see that the bubble integral functions contribute to the UV poles and the remaining triangle and box integral functions comprise the IR singularities.

The UV singularities are related to $\epsilon^{-1}$ poles. For one-loop amplitudes this divergence can be renormalised by the counter term in the $\overline{\mathrm{MS}}$ scheme [32],

$$
\left.\mathcal{A}_{n}^{(1)}\right|_{U V}=-\frac{(n-2)(4 \pi)^{\epsilon}}{2 \epsilon \Gamma(1-\epsilon)}\left(\frac{g}{4 \pi}\right)^{2} \beta_{0} \mathcal{A}_{n}^{(0)},
$$

where $\beta_{0}=\left(11 N_{c}\right) / 3$ in pure Yang-Mills.

An alternative way to view the UV divergences of an amplitude is by considering an expansion in the coupling constant, adding a counterterm given by,

$$
g \rightarrow g+\delta g
$$

Considering the pertubative expansion of a gluon amplitude with a non-vanishing tree-level amplitude,

$$
\mathcal{A}_{n}=\left(\frac{g}{4 \pi}\right)^{n-2} \mathcal{A}_{n}^{(0)}+\left(\frac{g}{4 \pi}\right)^{n} \mathcal{A}_{n}^{(1)}+\left(\frac{g}{4 \pi}\right)^{n+2} \mathcal{A}_{n}^{(2)}+\ldots
$$

we see that we require expansions of the form,

$$
(g+\delta g)^{i}=g^{i}+i g^{i-1} \delta g+\ldots .
$$

Applying the expansion (2.120) to the pertubative expression of the amplitude gives,

$$
\begin{aligned}
\mathcal{A}_{n}= & \left(\frac{g}{4 \pi}\right)^{n-2} \mathcal{A}_{n}^{(0)}+\left(\frac{g}{4 \pi}\right)^{n} \mathcal{A}_{n}^{(1)}+\left(\frac{g}{4 \pi}\right)^{n+2} \mathcal{A}_{n}^{(2)} \\
& +(n-2)\left(\frac{g}{4 \pi}\right)^{n-3} \delta g \mathcal{A}_{n}^{(0)}+n\left(\frac{g}{4 \pi}\right)^{n-1} \delta g \mathcal{A}_{n}^{(1)}+(n+2)\left(\frac{g}{4 \pi}\right)^{n+1} \delta g \mathcal{A}_{n}^{(2)}+\ldots .
\end{aligned}
$$




\section{REVIEW OF TECHNIQUES FOR CALCULATING SCATTERING AMPLITUDES}

The counterterm $\delta g$ is identified by considering the general form of the UV counterterm given by (2.117) and noticing that it is proportional to the tree-level amplitude. Taking the UV divergent part of the one-loop amplitude 1 and the tree-level term proportional to $\delta g$ for the moment, we have that the one-loop divergence is cancelled by

$$
\left.\left(\frac{g}{4 \pi}\right)^{n} \mathcal{A}_{n}^{(1)}\right|_{U V}+(n-2)\left(\frac{g}{4 \pi}\right)^{n-3} \frac{\delta g}{\epsilon} \mathcal{A}_{n}^{(0)}=\left(\frac{g}{4 \pi}\right)^{n}\left[\left.\mathcal{A}_{n}^{(1)}\right|_{U V}+(n-2)\left(\frac{g}{4 \pi}\right)^{-3} \frac{\delta g}{\epsilon} \mathcal{A}_{n}^{(0)}\right]=0,
$$

such that,

$$
\left.\mathcal{A}_{n}^{(1)}\right|_{U V}=-(n-2)\left(\frac{g}{4 \pi}\right)^{-3} \frac{\delta g}{\epsilon} \mathcal{A}_{n}^{(0)}
$$

From (2.117), we have

$$
(n-2)\left(\frac{g}{4 \pi}\right)^{-3} \frac{\delta g}{\epsilon} \mathcal{A}_{n}^{(0)}=\frac{(n-2)(4 \pi)^{\epsilon}}{2 \epsilon \Gamma(1-\epsilon)}\left(\frac{g}{4 \pi}\right)^{2} \beta_{0} \mathcal{A}_{n}^{(0)},
$$

such that the counter term $\delta g$ is given by,

$$
\delta g=\frac{(4 \pi)^{\epsilon}}{2 \Gamma(1-\epsilon)}\left(\frac{g}{4 \pi}\right)^{5} \beta_{0} .
$$

For the two-loop amplitudes whose tree-level amplitudes vanish, such as the twoloop all-plus, the one-loop amplitudes are rational and the first UV divergence in the theory appears at two-loop. The pertubative expansion is

$$
\mathcal{A}_{n}=\left(\frac{g}{4 \pi}\right)^{n} \mathcal{A}_{n}^{(1)}+\left(\frac{g}{4 \pi}\right)^{n+2} \mathcal{A}_{n}^{(2)}+\ldots
$$

applying (2.120),

$\mathcal{A}_{n}=\left(\frac{g}{4 \pi}\right)^{n} \mathcal{A}^{(1)}+\left(\frac{g}{4 \pi}\right)^{n+2} \mathcal{A}_{n}^{(2)}+n\left(\frac{g}{4 \pi}\right)^{n-1} \delta g \mathcal{A}_{n}^{(1)}+(n+2)\left(\frac{g}{4 \pi}\right)^{n+1} \delta g \mathcal{A}_{n}^{(2)}+\ldots$

\footnotetext{
${ }^{1}$ proportional to $\epsilon^{-1}$
} 
The UV divergence at two-loop is proportional to the one-loop amplitudes, as such we can group the divergent part of the two-loop amplitude together with the counterterm proportional to the one-loop amplitude,

$$
\left(\frac{g}{4 \pi}\right)^{n+2}\left[\left.\mathcal{A}_{n}^{(2)}\right|_{U V}+n\left(\frac{g}{4 \pi}\right)^{-3} \frac{\delta g}{\epsilon} \mathcal{A}_{n}^{(1)}\right]=0 .
$$

Since $\delta g$ has already been fixed, we have that the UV counterterm for this case is given by,

$$
\left.\mathcal{A}_{n}^{(2)}\right|_{U V}=-n\left(\frac{g}{4 \pi}\right)^{-3} \frac{\delta g}{\epsilon} \mathcal{A}_{n}^{(1)}=-n \frac{(4 \pi)^{\epsilon}}{2 \epsilon \Gamma(1-\epsilon)} \beta_{0}\left(\frac{g}{4 \pi}\right)^{2} \mathcal{A}_{n}^{(1)} .
$$

The collinear singularities arise from self energy corrections to the external lines and have the form [32],

$$
\left.\mathcal{A}_{n}^{(1)}\right|_{\text {collinear }}=-\left(\frac{g}{4 \pi}\right)^{2} \frac{n \gamma(g)}{\epsilon} \mathcal{A}_{n}^{(0)}
$$

where $\gamma(g)=11 N_{c} / 6$ for pure Yang-Mills.

For the case where the tree-level amplitudes vanish in the theory, the collinear divergences of the two-loop amplitude are given by,

$$
\left.\mathcal{A}_{n}^{(2)}\right|_{\text {collinear }}=-\left(\frac{g}{4 \pi}\right)^{2} \frac{n \gamma(g)}{\epsilon} \mathcal{A}_{n}^{(1)} .
$$

Interestingly, in the two-loop all-plus amplitude, the collinear and UV divergences are given by (2.129) and (2.131), which cancel upon expanding in $\epsilon$. We only consider unrenormalised amplitudes in this thesis, and the analysis of UV and collinear divergences for the two-loop all-plus amplitude above shows that it contains only soft IR divergences.

The IR singular structure of loop amplitudes has been investigated by [33], where the highest order IR divergence of a loop amplitude is

$$
\mathcal{A}_{n}^{(L)} \sim\left(\frac{1}{\epsilon}\right)^{2 L}
$$




\section{REVIEW OF TECHNIQUES FOR CALCULATING SCATTERING AMPLITUDES}

Therefore a one-loop amplitude in pure Yang-Mills has at most double poles in the dimensional regulator. Additionally, the soft divergences arise from the virtual contribution whereby a soft gluon connects two external legs. For a soft gluon connecting two external legs, $i$ and $j$, we pick up a factor proportional to

$$
-\frac{1}{\epsilon^{2}}\left(-\frac{\mu^{2}}{s_{i j}}\right)^{\epsilon}
$$

Considering all the possible hard legs that can be connected with a soft gluon, the full soft contribution can be obtained. This is easily seen by viewing the form of the IR singularity structure of the leading in colour two-loop five-point all-plus amplitude, since we know it only contains soft IR divergences. For this amplitude, the colour ordering imposes that the external legs have a fixed cyclic ordering. Contributions to the soft divergences are therefore only obtained by connecting adjacent legs with a soft gluon, leading to the result,

$$
\left.A_{5: 1}^{(2)}\left(a^{+}, b^{+}, c^{+}, d^{+}, e^{+}\right)\right|_{I R}=-\frac{1}{\epsilon^{2}} \sum_{i=1}^{5}\left(-\frac{\mu^{2}}{s_{i i+1}}\right)^{\epsilon} A_{5}^{(1)}\left(a^{+}, b^{+}, c^{+}, d^{+}, e^{+}\right) .
$$

Combining our understanding of the amplitudes integral basis and the nature of their singularities provides a major advantage in the pursuit of the calculation of one-loop amplitudes. The integral functions evaluate to singular terms in epsilon and polylogarithms whose arguments are Lorentz invariant scalar products of the external momenta. These terms contain branch cut singularities and thus constrain the branch cut singularity structure of the amplitude, paving the way for a modern application of the $S$-matrix program philosophy [46].

\subsection{Exploiting the Singularity Structure of Loop Amplitudes: Unitarity and Branch Cuts}

The $S$-matrix is a mathematical object that describes the scattering behaviour of momentum eigenstates propagating from $t=-\infty$ to $t=+\infty$ and scattering 


\subsection{Exploiting the Singularity Structure of Loop Amplitudes: Unitarity and Branch Cuts}

in some finite time,

$$
S=\mathbb{1}+i T
$$

The principal term in 2.135 encodes the possibility that the initial states do not meet at the same spacetime point and thus do not interact. The latter term governs the interactions of the states for the case in which they scatter.

A scattering amplitude can be defined through an element of the $S$-matrix whose square is related to the probability of such an interaction occurring

$$
\left\langle p_{n} \cdots p_{i+1}|S| p_{1} \cdots p_{i}\right\rangle=A_{n}\left(p_{1}, \ldots, p_{i} ; p_{i+1}, \ldots, p_{n}\right)
$$

where the momenta on the right hand side of the equation are partitioned to emphasise the incoming and outgoing momenta. The sum of the probabilities of all possible outcomes occurring is 1 , imposing the constraint that the $S$-matrix is unitary,

$$
S S^{\dagger}=\mathbb{1}
$$

Substituting (2.135) in (2.137) gives a condition on the interaction matrix $T$,

$$
T-T^{\dagger}=T T^{\dagger}
$$

The left-hand side corresponds to $2 \operatorname{Im}(T)$, but noting that $T$ and $T^{\dagger}$ are related to each other by hermitian analyticity [46] implies $2 \operatorname{Im}(T)=\operatorname{Disc}(T)$, where $\operatorname{Disc}(T)$ is a discontinuity across a branch cut. The right-hand side is the product of interaction matrices summed over all possible internal states.

Expanding the interaction matrix in the coupling constant,

$$
T=a T^{(0)}+a^{2} T^{(1)}+a^{3} T^{(2)}+\ldots
$$

where $a \propto g^{2}$, and collecting terms that contribute at the same order in the coupling, we see that

$$
i\left(T^{(1)}-T^{(1) \dagger}\right)=T^{(0)} T^{(0) \dagger} .
$$




\section{REVIEW OF TECHNIQUES FOR CALCULATING SCATTERING AMPLITUDES}

Therefore, the discontinuity across a branch cut of one-loop amplitudes is related to the on-shell product of tree-level amplitudes summed over all possible configurations of the intermediate on-shell legs. This is the basis for the modern technique of unitarity, used to construct loop level amplitudes in terms of lower order loop amplitudes.

\subsubsection{The Unitarity Method}

The description of a discontinuity of an amplitude in terms of a product of lower order products of amplitudes was known to researchers in the early stages of the $S$-matrix program [46]. The left-hand side of (2.140) can be calculated using Cutkosky's rules [47]. In the context of a one-loop amplitude, this amounts to cutting two internal propagators using the replacement

$$
\frac{i}{\ell^{2}} \rightarrow i(2 \pi)^{4} \delta^{(+)}\left(\ell^{2}\right), \quad \frac{i}{\left(\ell-p_{i j}\right)^{2}} \rightarrow i(2 \pi)^{4} \delta^{(+)}\left(\left(\ell-p_{i j}\right)^{2}\right) .
$$

The double cut factorises the one-loop amplitude into a product of two on-shell trees with a sum over the helicities of the cut legs. In the original $S$-matrix program, this was used in the context of determining the amplitude using dispersion relations. The unitary relation (2.140) was used to simplify the numerator of the dispersion integrals - the amplitude written in terms of the complex variable introduced by Cauchy's integral formula. A major issue with this approach is that the lower order amplitudes in the numerator are also determined using dispersion relations. Therefore, generalised dispersion relations were needed in order to capture the dispersive part of the amplitude from the absorptive part Without further constraints, this approach to calculating amplitudes is unfeasible.

The development of QCD gave a description of loop amplitudes in terms of Feynman integrals that ultimately lead to a way of circumventing dispersion relations. At one-loop,the four dimensional integral reduction techniques [50, 51, 54, 55 we have reviewed, describe all one-loop amplitudes in terms of a basis of integral functions. The scalar integrals in the basis evaluate to polylogarithmic

\footnotetext{
${ }^{1}$ given by the discontinuity
} 


\subsection{Exploiting the Singularity Structure of Loop Amplitudes: Unitarity and Branch Cuts}

functions that contain branch cut singularities, thus isolating the branch cut singularity structure of the one-loop amplitudes.

Constraining the singularity structure allows for a feasible application of the $S$-matrix approach using the unitarity approach [53, 56]. In the unitarity method, Cutkosky's rules are used in reverse. Sewing tree-level amplitudes with on-shell internal legs in a particular channel describes the corresponding discontinuity of the one-loop amplitude. The authors showed in refs. [53, 56] that promoting the cut integral to a covariant integral with the opposite replacement of (2.141) produces integrals that correspond to those of the integral basis, multiplied by rational coefficients. Systematically repeating this process with cuts in all the possible channels of an amplitude fully reconstructs the integral basis determining their rational coefficients. Note that for amplitudes that contain rational terms, the cuts in four dimensions do not fully determine the amplitude.

The choice available to us at the stage of imposing the cuts has large implications in how the amplitude is determined. The cuts can be performed in $D=4$ dimensions, or $D=4-2 \epsilon$-dimensions. Both methods have their pros and cons. Four-dimensional unitarity is the method used in this thesis, but we discuss $D$ dimensional unitarity briefly at the end of this section.

The delta functions that impose the cuts are in four-dimensions, such that the $-2 \epsilon$ dimensional part of the cut loop momenta are set to zero. The loop integration and the propagators are still in $D$-dimensions, in order to regularise the integral. This may seem like some vital information is lost, however discrepancies that arise from this method contribute to rational terms at $\mathcal{O}\left(\epsilon^{0}\right)[53]$. This $\mathcal{O}\left(\epsilon^{0}\right)$ discrepancy will be recovered when we determine the rational piece via complex recursion in subsection 2.10.1. The part of the amplitude that is recovered from analysing the cuts is called the cut constructible part of the amplitude.

As a small example of the unitarity method, consider the leading in colour one-loop four-gluon MHV amplitude. The integral basis for this amplitude is 


\section{REVIEW OF TECHNIQUES FOR CALCULATING SCATTERING AMPLITUDES}

given by

$$
A_{4}^{(1)}\left(1^{-}, 2^{-}, 3^{+}, 4^{+}\right)=c_{4} I_{4}^{1 m}+\sum_{i} c_{3, i}^{2 m} I_{3}^{2 m}+\sum_{i} c_{3, i}^{1 m} I_{3}^{1 m}+\sum_{i} c_{2, i} I_{2}^{2 m}
$$

Performing a double cut on the amplitude in the $s$-channel gives the discontinuity of the amplitude in terms of the following cut integral,

$$
\left.A_{4}^{(1)}\left(1^{-}, 2^{-}, 3^{+}, 4^{+}\right)\right|_{s-\text { channel }}=\int d \operatorname{LIPS} A_{4}^{(0)}\left(1^{-}, 2^{-}, \ell_{1}^{+},-\ell_{2}^{+}\right) A_{4}^{(0)}\left(\ell_{2}^{-},-\ell_{1}^{-}, 3^{+}, 4^{+}\right),
$$

where $d L I P S$ is the Lorentz invariant phase space measure defined as $d L I P S=$ $d^{D} \ell_{1} \delta^{(+)}\left(\ell_{1}^{2}\right) \delta^{(+)}\left(\ell_{2}^{2}\right) \delta^{(4)}\left(p_{12}+l_{1}-l_{2}\right)^{1}$, encoding the loop integral measure and the cut conditions. The other helicity configurations of the internal on-shell legs give vanishing contributions to the cuts, since the tree-level amplitudes vanish.

Integral functions containing a higher number of propagators will contribute to this discontinuity since they also contain the propagators that are placed onshell. Hence from examining the double cuts, we obtain information about the discontinuities originating from all the integral functions in the basis.

Promoting the cut integral (2.143) to a covariant integral,

$$
\begin{aligned}
\left.A_{4}^{(1)}\left(1^{-}, 2^{-}, 3^{+}, 4^{+}\right)\right|_{s-\text { channel }} & =\int \frac{d^{D} \ell_{1}}{(2 \pi)^{D}} \frac{1}{\ell_{1}^{2} \ell_{2}^{2}} A_{4}^{(0)}\left(1^{-}, 2^{-}, \ell_{1}^{+},-\ell_{2}^{+}\right) A_{4}^{(0)}\left(\ell_{2}^{-},-\ell_{1}^{-}, 3^{+}, 4^{+}\right) \\
& =\int \frac{d^{D} \ell_{1}}{(2 \pi)^{D}} \frac{1}{\ell_{1}^{2} \ell_{2}^{2}} \frac{\langle 12\rangle^{3}}{\left\langle 2 \ell_{1}\right\rangle\left\langle\ell_{1} \ell_{2}\right\rangle\left\langle\ell_{2} 1\right\rangle} \frac{\left\langle\ell_{2} \ell_{1}\right\rangle^{3}}{\left\langle\ell_{1} 3\right\rangle\langle 34\rangle\left\langle 4 \ell_{2}\right\rangle} \\
& =\frac{\langle 12\rangle^{3}}{\langle 34\rangle} \int \frac{d^{D} \ell_{1}}{(2 \pi)^{D}} \frac{1}{\ell_{1}^{2} \ell_{2}^{2}} \frac{\left[1\left|\ell_{2}\right| \ell_{1}\right\rangle\left\langle\ell_{1}\left|\ell_{2}\right| 4\right]}{\left\langle 2 \ell_{1}\right\rangle\left\langle\ell_{1} 3\right\rangle\left[1\left|\ell_{2}\right| 1\right\rangle\left[4\left|\ell_{2}\right| 4\right\rangle} \\
& =\frac{\langle 12\rangle^{3}[12][34]}{\langle 34\rangle} \int \frac{d^{D} \ell_{1}}{(2 \pi)^{D}} \frac{1}{\ell_{1}^{2} \ell_{2}^{2}} \frac{1}{\left[1\left|\ell_{2}\right| 1\right\rangle\left[4\left|\ell_{2}\right| 4\right\rangle} .
\end{aligned}
$$

The covariant integral now represents a scalar box integral. Reimposing the cuts and performing some simplifications, we find the coefficient of the $s$-channel cut

\footnotetext{
${ }^{1}$ where, $\ell_{2}=\ell_{1}+p_{12}$.
} 


\subsection{Exploiting the Singularity Structure of Loop Amplitudes: Unitarity and Branch Cuts}

to be,

$$
\left.A_{4}^{(1)}\left(1^{-}, 2^{-}, 3^{+}, 4^{+}\right)\right|_{\text {s-channel }}=\langle 12\rangle^{2}[34]^{2} .
$$

This has shown that the $s$-channel cut uniquely determines the coefficient of the box integral function contained in the full amplitude. We confirm this statement by considering quadruple cuts of the amplitude in the following section.

We have only considered the $s$-channel cut here. In a practical calculation, we should consider the $t$-channel cut too, which contains additional triangle and bubble integrals.

Double cuts of a one-loop amplitude can thus determine the amplitude with the help of the known integral basis. Generalised unitarity [30] extends the use of cuts such that the cut constructible calculation is relatively easier.

\subsubsection{Generalised Unitarity}

It is possible to consider cutting more than two propagators at a time when deducing the cut constructible parts of an amplitude. This method is known as generalised unitarity and has its roots in the original $S$-matrix program [46]. However, its more familiar form came about from calculating coefficients of box integral functions in $\mathcal{N}=4 \mathrm{SYM}$ [30]. Cutting more than two propagators at once has the advantage of isolating specific contributions of various integral functions to a discontinuity.

At one-loop we have four degrees of freedom encoded by the loop momentum that we are required to integrate over. The integral basis contains integrals that have at most four propagators. Double cuts pick up the discontinuities of bubble, triangle and box integral functions. Alternatively, quadruple cuts isolate contributions to the discontinuity from box integral functions and triple cuts have contributions from box and triangle integral functions. In this way we may view the integral basis as having a hierarchy of branch cut structures.

When all four of the box integral functions propagators are placed on shell, the branch cut structure found by the cuts is isolated to the box functions since 


\section{REVIEW OF TECHNIQUES FOR CALCULATING SCATTERING AMPLITUDES}

the triangles and bubbles vanish,

$$
\left.\operatorname{Disc}\left(A_{n}^{(1)}\right)\right|_{\text {quad cut }}=\sum_{i} c_{4, i} \operatorname{Disc}\left(I_{4}\right)
$$

By imposing the quadruple cuts, the loop momentum has four constraints places upon it, freezing the loop integration and uniquely determining the box coefficient.

Taking the four-point example we examined earlier the quadruple cut of $A^{(1)}\left(1^{-}, 2^{-}, 3^{+}, 4^{+}\right)$gives the coefficient,

$c_{4}=\int d \operatorname{LIPSA_{3}^{(0)}}\left(1^{-}, \ell_{2}^{-},-\ell_{1}^{+}\right) A_{3}^{(0)}\left(\ell_{3}^{+},-\ell_{2}^{+}, 2^{-}\right) A_{3}^{(0)}\left(\ell_{4}^{-}, \ell_{3}^{-}, 3^{+}\right) A_{3}^{(0)}\left(-\ell_{4}^{+}, 4^{+}, \ell_{1}^{-}\right)$,

where the integral is frozen due to the four delta functions, and all other internal helicity configurations vanish. Examining the cut conditions, i.e. $\ell_{i}^{2}=0$ for $i=1,2,3,4$, the necessity for having $p_{i} \in \mathbb{C}$ becomes apparent.

$$
\ell_{4}^{2}=\left(\ell_{1}+p_{4}\right)^{2}=\left\langle\ell_{1} 4\right\rangle\left[4 \ell_{1}\right]=0,
$$

since we require $A_{3}^{(0)}\left(-\ell_{4}^{+}, 4^{+}, \ell_{1}^{-}\right)$to be non-zero we pick $\lambda_{\ell_{1}}=\lambda_{4}$. There were two choices available to us, leading to two sets of solutions to the cut conditions. We choose the non-trivial solution. Performing the same analysis on the corner containing $p_{1}$,

$$
\ell_{2}^{2}=\left(\ell_{1}-p_{1}\right)^{2}=\left\langle\ell_{1} 1\right\rangle\left[1 \ell_{1}\right]=0
$$

and we select, up to a scale factor, $\bar{\lambda}_{\ell_{1}}=\alpha_{\ell_{1}} \bar{\lambda}_{1}$. Fixing the scale factor across a massive corner,

$$
\ell_{3}^{2}=\left(\ell_{1}+p_{34}\right)^{2}=0, \quad s_{34}+\alpha_{\ell_{1}}\left\langle 4\left|p_{34}\right| 1\right]=0 \quad \Longrightarrow \quad \alpha_{\ell_{1}}=\frac{\langle 12\rangle}{\langle 42\rangle},
$$




\subsection{Exploiting the Singularity Structure of Loop Amplitudes: Unitarity and Branch Cuts}

hence

$$
\ell_{1}=\frac{\langle 12\rangle}{\langle 42\rangle} \lambda_{4} \bar{\lambda}_{1}
$$

Solving for the remaining loop momenta gives the solutions,

$$
\ell_{1}=\frac{\langle 12\rangle}{\langle 42\rangle} \lambda_{4} \bar{\lambda}_{1}, \quad \ell_{2}=\frac{\langle 14\rangle}{\langle 42\rangle} \lambda_{2} \bar{\lambda}_{1}, \quad \ell_{3}=\frac{\langle 34\rangle}{\langle 24\rangle} \lambda_{2} \bar{\lambda}_{3}, \quad \ell_{4}=\frac{\langle 23\rangle}{\langle 42\rangle} \lambda_{4} \bar{\lambda}_{3}
$$

we arrive at the same expression for the one-loop massless box integral,

$$
c_{4}=\langle 12\rangle^{2}[34]^{2},
$$

and we have,

$$
\left.A_{4}^{(1)}\left(1^{-}, 2^{-}, 3^{+}, 4^{+}\right)\right|_{\text {quad-cut }}=\langle 12\rangle^{2}[34]^{2} .
$$

Next, by considering triple cuts of the triangle integral functions, we will pick up contributions from triangle integral functions as well as box integral functions.

$$
\left.\operatorname{Disc}\left(A_{n}^{(1)}\right)\right|_{\text {triple cut }}=\sum_{i} c_{4, i} \operatorname{Disc}\left(I_{4}\right)+\sum_{i} c_{3, i} \operatorname{Disc}\left(I_{3}\right)
$$

Since we have determined the box contributions from the quadruple cuts, any box integral that we pick up from performing the triple cuts can be discarded. At the level of the triple cuts, there is one degree of freedom left unfixed after imposing the cuts. Determining the coefficients in this case require explicit integration over this remaining degree of freedom. One can use complex analysis to determine the coefficients [59] or by using the canonical basis approach [60] that provides a dictionary for integrals appearing at the level of triple-cuts or double cuts. The canonical basis approach is the method of choice in all subsequent calculations of integral coefficients that require integration. The bubble contributions can be determined in a similar fashion.

By performing the cuts in all channels the full cut constructible part of an 


\section{REVIEW OF TECHNIQUES FOR CALCULATING SCATTERING AMPLITUDES}

amplitude can be determined.

\subsubsection{D-dimensional Unitarity}

The unitarity sections above performed cuts of the amplitude in $D=4$ dimensions. The simplicity of the calculation, supplied by ability to use fourdimensional amplitudes in the cut integrands, came at the cost of an $\mathcal{O}\left(\epsilon^{0}\right)$ ambiguity. Alternatively, cuts of the amplitude can be performed in $D=4-2 \epsilon$ dimensions [61 66] with a decomposition of the loop momentum in terms of a four-dimensional component and a $-2 \epsilon$ component

$$
\ell=\ell^{[4]}+\ell^{[-2 \epsilon]}=\tilde{\ell}+\mu,
$$

where $\ell^{2}=\tilde{\ell}^{2}-\mu^{2}$. The decomposition of the $D$-dimensional integral measure then reads as,

$$
\int \frac{d^{D} \ell}{(2 \pi)^{D}}=\int \frac{d^{4} \tilde{\ell}}{(2 \pi)^{4}} \int \frac{d^{-\epsilon} \mu^{2}}{(2 \pi)^{-2 \epsilon}} .
$$

This framework avoids any ambiguity in the dimensional regulator leading to, in part, all-order collinear behaviour of loop-amplitudes [140] and all- $\epsilon$ forms of one-loop amplitudes 141 .

In order to use the cuts in $D$-dimensions, a $D$-dimensional basis of integrals is required [67, 68]. A major advantage of this line of work amounts to a determination of the rational parts of the amplitudes that arise from rational integrals involving $\mathcal{O}(-2 \epsilon)$ integrations. The contributions to the rational terms in oneloop amplitudes were determined directly in [71].

More recently, the $D$-dimensional unitarity approach has proved to be an especially effective method in computing two-loop amplitudes [94 96, 99 102, 105, 106, 108 110.

Despite the reach and obvious effectiveness of $D$-dimensional unitarity 1 a major drawback is the ability to reach higher multiplicities due to the need for calculating more complicated integrals. With this in mind, our preferred method

\footnotetext{
${ }^{1}$ The first $S$-matrix elements at two-loops (at both leading in colour and full colour) with non-identical helicities have been calculated using $D$-dimensional unitarity.
} 


\subsection{Exploiting the Singularity Structure of Loop Amplitudes: Unitarity and Branch Cuts}

of four-dimensional unitarity avoids this issue especially for the two-loop all-plus amplitude 1 at the cost of calculating the rational pieces separately.

The following section discusses the factorisation properties of loop amplitudes, which are vital in determining the rational terms of loop amplitudes in the fourdimensional unitarity philosophy.

\subsubsection{Factorisation of Loop Amplitudes}

Much like their tree-level partners, loop amplitudes also exhibit factorisation properties. These properties are useful for consistency checks on an amplitude and also provide strong constraints in the process of building some rational oneloop amplitudes $[38,77,78$. In chapter 3 and chapter 4 we use the multiparticle factorisation of the two-loop all-plus amplitude to determine its rational contribution, as such we briefly review the factorisation theorems here.

When two external legs become collinear under the parametrisation (2.86), one-loop amplitudes factorise in a similar way to tree-level amplitudes with the addition of a term dependent on a one-loop splitting function [56, 140, 142],

$$
\begin{aligned}
A_{n}^{(1)}\left(\ldots, i^{h_{i}}, j^{h_{j}}, \ldots\right) \underset{i \| j}{\longrightarrow} \sum_{h= \pm}( & \operatorname{Split}_{-h}^{(0)}\left(i^{h_{i}}, j^{h_{j}} ; z\right) A_{n-1}^{(1)}\left(1 \ldots, k^{h}, \ldots, n\right) \\
& \left.+\operatorname{Split}_{-h}^{(1)}\left(i^{h_{i}}, j^{h_{j}} ; z\right) A_{n-1}^{(0)}\left(1 \ldots, k^{h}, \ldots, n\right)\right) .
\end{aligned}
$$

Explicitly, the one-loop splitting functions can be written as the sum of a factorising and non-factorising terms [142],

$$
\operatorname{Split}_{h}^{(1)}\left(i^{h_{i}}, j^{h_{j}}\right)=\operatorname{Split}_{h}^{(1)}\left(i^{h_{i}}, j^{h_{j}}\right)_{\text {fact }}+\operatorname{Split}_{h}^{(1)}\left(i^{h_{i}}, j^{h_{j}}\right)_{\text {non-fact }} .
$$

\footnotetext{
${ }^{1}$ discussed in chapter 4 and chapter 3
} 


\section{REVIEW OF TECHNIQUES FOR CALCULATING SCATTERING AMPLITUDES}

The factorising parts are given as,

$$
\begin{aligned}
& \operatorname{Split}_{ \pm}^{(1)}\left(i^{+}, j^{-}\right)_{\text {fact }}=\operatorname{Split}_{ \pm}^{(1)}\left(i^{-}, j^{+}\right)_{\mathrm{fact}}=0 \\
& \operatorname{Split}_{+}^{(1)}\left(i^{+}, j^{+}\right)_{\mathrm{fact}}=-\frac{1}{3} \sqrt{z(1-z)} \frac{[i j]}{\langle i j\rangle^{2}} \\
& \operatorname{Split}_{-}^{(1)}\left(i^{+}, j^{+}\right)_{\text {fact }}=\frac{1}{3} \sqrt{z(1-z)} \frac{1}{\langle i j\rangle},
\end{aligned}
$$

and the non-factorising part is given as,

$\operatorname{Split}_{h}^{(1)}\left(i^{h_{i}}, j^{h_{j}}\right)_{\text {non-fact }}=\operatorname{Split}_{h}^{(0)}\left(i^{h_{i}}, j^{h_{j}}\right)\left[-\frac{1}{\epsilon^{2}}\left(-\frac{z(1-z) s_{i j}}{\mu^{2}}\right)^{-\epsilon}+2 \ln (z) \ln (1-z)-\frac{\pi^{2}}{6}\right]$.

The one-loop collinear behaviour has a straightforward generalisation to allloop orders [58, 140],

$$
A_{n}^{(L)}\left(\ldots, i^{h_{i}}, j^{h_{j}}, \ldots\right) \underset{i \| j}{\longrightarrow} \sum_{l=0}^{L} \sum_{h= \pm} \operatorname{Split}_{-h}^{(l)}\left(i^{h_{i}}, j^{h_{j}} ; z\right) A_{n-1}^{(L-l)}\left(\ldots, k^{h}, \ldots\right)
$$

where the two-loop splitting functions were found in ref. [58]. Analogous expressions exist for soft factorisations in the limit that the momentum of an external leg approaches zero. The soft behaviour of gluon amplitudes has been understood at tree-level [133, 134], one-loop [142, 143] and two-loop [144 147].

Likewise for multiparticle factorisations, amplitudes factorise in the limit, $A_{n}^{(L)}(1, \ldots, i, i+1, \ldots, n) \underset{k^{2}=t_{1 \ldots, i} \rightarrow 0}{\longrightarrow} \sum_{l=0}^{L} \sum_{h= \pm} A_{i+1}^{(l)}\left(1, \ldots i, k^{h}\right) \frac{-1}{k^{2}} A_{n-i+1}^{(L-l)}\left(-k^{-h}, i+1, \ldots, n\right)$

which is of paramount importance in calculating the rational part of loop-amplitudes through recursive techniques.

A specific example of its use is presented in section 3.4 and section 4.6 where the rational parts of some two-loop all-plus amplitudes are calculated, both at leading in colour [88] and subleading in colour [89] respectively. In those cases, 
we examine the factorisations of the amplitudes and only consider the rational contributions to the factorised amplitudes in such a limit. The multiparticle and collinear ${ }^{1}$ factorisations used in this way allow us to calculate the rational part of a higher-point amplitude using lower-point amplitudes through augmented recursion. The multiparticle factorisations contain only simple poles, but the collinear factorisations contain double poles that hinder the canonical implementation of complex recursion. We discuss this issue and show how to overcome it in subsection 2.10.1. Preliminarily, in the next section we discuss rational amplitudes and some of their properties.

\subsection{Rational Contributions to Loop Amplitudes}

This section deals with rational contributions to loop amplitudes. In particular we consider the rational one-loop all-plus and single-minus helicity amplitudes, then in the later sections we review the rational contributions to the two-loop all-plus amplitude at $n=5$ through augmented recursion. The one-loop all-plus and single-minus helicity amplitudes are completely rational, i.e. they are finite as $\epsilon \rightarrow 0$ and additionally do not contain branch cut singularities in $D=4$ dimensions. These helicity amplitudes owe their simpler singular structure ${ }^{2}$ to the fact that their tree-level counterparts vanish through supersymmetric Ward identities 72 74].

The leading in colour one-loop all-plus amplitude [77 79 expressed to $\mathcal{O}\left(\epsilon^{0}\right)$ is,

$$
A_{n: 1}^{(1)}\left(1^{+}, 2^{+}, \cdots, n^{+}\right)=-\frac{i}{3} \sum_{1 \leq k_{1}<k_{2}<k_{3}<k_{4} \leq n} \frac{\left\langle k_{1} k_{2}\right\rangle\left[k_{2} k_{3}\right]\left\langle k_{3} k_{4}\right\rangle\left[k_{4} k_{1}\right]}{\langle 12\rangle\langle 23\rangle \cdots\langle n 1\rangle}+O(\epsilon) .
$$

This expression is rational to order $\epsilon^{0}$.

The one-loop $n$-point single-minus amplitude was found recursively [79] via a generalised of the Berends-Giele recursion method, and their forms for four-,

\footnotetext{
${ }^{1}$ Which is a special case of the multiparticle factorisation.

${ }^{2}$ compared to other one-loop amplitudes.
} 


\section{REVIEW OF TECHNIQUES FOR CALCULATING SCATTERING AMPLITUDES}

five- and six-point [38] are given as

$$
\begin{aligned}
& A_{4: 1}^{(1)}\left(a^{-}, b^{+}, c^{+}, d^{+}\right)=\frac{i}{3} \frac{[b d]^{3}\langle b d\rangle}{[a b]\langle c d\rangle[d a]}, \\
& A_{5: 1}^{(1)}\left(a^{-}, b^{+}, c^{+}, d^{+}, e^{+}\right)=\frac{i}{3} \frac{1}{\langle c d\rangle^{2}}\left(-\frac{[b e]^{3}}{[a b][e a]}+\frac{\langle a d\rangle^{3}[d e]\langle c e\rangle}{\langle a b\rangle\langle b c\rangle\langle d e\rangle^{2}}+\frac{\langle a c\rangle^{3}[b c]\langle b d\rangle}{\langle a e\rangle\langle d e\rangle\langle b c\rangle^{2}}\right), \\
& A_{6: 1}^{(1)}\left(a^{-}, b^{+}, c^{+}, d^{+}, e^{+}, f^{+}\right) \\
& =\frac{i}{3}\left[\frac{\left[f\left|K_{b c}\right| a\right\rangle^{3}}{\langle a b\rangle\langle b c\rangle\langle d e\rangle^{2} t_{a b c}\left[f\left|K_{a b}\right| c\right\rangle}+\frac{\left[b\left|K_{c d}\right| a\right\rangle^{3}}{\langle c d\rangle^{2}\langle e f\rangle\langle f a\rangle t_{b c d}\left[b\left|K_{c d}\right| e\right\rangle}\right. \\
& +\frac{[b f]^{3}}{[a b][f a] t_{c d e}}\left(\frac{[b c][c d]}{\langle d e\rangle\left[b\left|K_{c d}\right| e\right\rangle}-\frac{[d e][e f]}{\langle c d\rangle\left[f\left|K_{a b}\right| c\right\rangle}+\frac{[c e]}{\langle c d\rangle\langle d e\rangle}\right) \\
& -\frac{\langle a c\rangle^{3}[b c]\langle b d\rangle}{\langle b c\rangle^{2}\langle c d\rangle^{2}\langle d e\rangle\langle e f\rangle\langle f a\rangle}+\frac{\langle a e\rangle^{3}[e f]\langle d f\rangle}{\langle a b\rangle\langle b c\rangle\langle c d\rangle\langle d e\rangle^{2}\langle e f\rangle^{2}} \\
& \left.-\frac{\langle a d\rangle^{3}\langle c e\rangle\left[d\left|K_{b c}\right| a\right\rangle}{\langle a b\rangle\langle b c\rangle\langle c d\rangle^{2}\langle d e\rangle^{2}\langle e f\rangle\langle f a\rangle}\right] \text {. }
\end{aligned}
$$

All- $\epsilon$ forms of the one-loop amplitudes exist and are expressed in terms of higher dimensional scalar integrals. For $n \leq 6$ they are 64

$$
\begin{aligned}
& A_{4: 1}^{(1)}\left(1^{+}, 2^{+}, 3^{+}, 4^{+}\right)=\frac{2 i \epsilon(1-\epsilon)}{\langle 12\rangle\langle 23\rangle\langle 34\rangle\langle 41\rangle} \times s_{12} s_{23} I_{4}^{D=8-2 \epsilon}, \\
& A_{5: 1}^{(1)}\left(1^{+}, 2^{+}, 3^{+}, 4^{+}, 5^{+}\right)=\frac{i \epsilon(1-\epsilon)}{\langle 12\rangle\langle 23\rangle\langle 34\rangle\langle 45\rangle\langle 51\rangle} \\
& \times\left[s_{23} s_{34} I_{4}^{(1), D=8-2 \epsilon}+s_{34} s_{45} I_{4}^{(2), D=8-2 \epsilon}+s_{45} s_{51} I_{4}^{(3), D=8-2 \epsilon}\right. \\
&\left.+s_{51} s_{12} I_{4}^{(4), D=8-2 \epsilon}+s_{12} s_{23} I_{4}^{(5), D=8-2 \epsilon}+(4-2 \epsilon) \varepsilon(1,2,3,4) I_{5}^{D=10-2 \epsilon}\right],
\end{aligned}
$$




$$
\begin{aligned}
& A_{6: 1}^{(1)}\left(1^{+}, 2^{+}, 3^{+}, 4^{+}, 5^{+}, 6^{+}\right)=\frac{i \epsilon(1-\epsilon)}{\langle 12\rangle\langle 23\rangle\langle 34\rangle\langle 45\rangle\langle 56\rangle\langle 61\rangle} \frac{1}{2}[ \\
& -\sum_{1 \leq i_{1}<i_{2} \leq 6} \operatorname{tr}\left[\not k_{i_{1}} \not P_{i_{1}+1, i_{2}-1} \not k_{i_{2}} \not P_{i_{2}+1, i_{1}-1}\right] I_{4}^{\left(i_{1}, i_{2}\right), D=8-2 \epsilon}+(4-2 \epsilon) \operatorname{tr}\left[\not p_{1} \not p_{2} \not p_{3} \not p_{4} \not p_{5} \not p_{6}\right] I_{6}^{D=10-2 \epsilon} \\
& \left.+(4-2 \epsilon) \sum_{i=1}^{6} \varepsilon(i+1, i+2, i+3, i+4) I_{5}^{(i), D=10-2 \epsilon}\right]
\end{aligned}
$$

where $I_{m}^{(i), D}$ denotes the $D$-dimensional scalar integral obtained by removing the loop propagator between legs $i-1$ and $i$ from the $(m+1)$-point scalar integral etc. [55]. The all- $\epsilon$ forms of the one-loop all-plus amplitudes are relevant as they appear as the coefficients of the IR divergent part of the leading in colour two-loop amplitudes [97]. Moreover, when taking collinear limits of the two-loop all-plus, the all- $\epsilon$ form of the one-loop amplitudes are required to correctly describe the collinear limits to all orders in $\epsilon$ [11].

The expression of the all- $\epsilon$ forms in terms of higher dimensional integrals is a reflection of the fact they contain cuts in higher dimensions. The higher dimensional cuts are related to the integrals arising from considering $-2 \epsilon$ dimensions when performing the integral reduction $[62,63,69,70]$.

With the relevant rational loop amplitudes discussed, we move to the preferred method of determining the rational part of loop amplitudes - augmented recursion.

\subsubsection{Complex Recursion Revisited: Augmented Recur- sion}

As touched on earlier, rational contributions to loop amplitudes may contain double poles as well as single poles. If we consider the case of an amplitude that only contains a simple pole at $z_{i}$, the function has a Laurent expansion,

$$
R_{s}(z)=\frac{a_{-1}}{\left(z-z_{i}\right)}+\mathcal{O}\left(\left(z-z_{i}\right)^{0}\right)
$$




\section{REVIEW OF TECHNIQUES FOR CALCULATING SCATTERING AMPLITUDES}

This is analogous to the tree-level case and we may determine the single pole contributions via the residue theorem and general factorisation theorems.

In general, amplitudes can contain simple poles, double poles and higher order poles. Amplitudes that contain double poles are slightly more complicated than their tree-level counterparts. We cannot simply apply the residue theorem and understand the full rational part of the amplitude by examining its residues using factorisation theorems. Factorisation theorems describe the contributions to the residue from leading singularities - the highest order poles. This is why simple pole contributions can be straightforwardly described by their residues. The higher order poles (double pole or above) have a Laurent series for which there are subleading poles. The subleading poles are non-factorising objects and must be determined by alternative methods. In this thesis, we choose to calculate these subleading poles present in the rational part of amplitudes which contain double poles using augmented recursion [81, 84 89]. Augmented recursion is a modified $\mathrm{BCFW}$ recursion technique.

We begin the discussion on augmented recursion generally with an analysis of a Laurent series of a function, $R(z)$, whose highest order pole is a double pole. Borrowing from the BCFW technique, we consider the ratio of this series with $z$, to relate the residue at $z=0$ to the rational function we wish to calculate. Then, we consider a general expression that can arise from a practical calculation of a rational term containing double poles and compare the residues from each expression. A specific calculation is then used to demonstrate augmented recursion, applying it to the case of the two-loop five-gluon all-plus rational piece.

Consider the Laurent expansion of the rational part of an amplitude that contains a double pole. Expanding about the point in the complex plane where the double poles are located, $z_{j}$, we have

$$
R(z)=\frac{b_{-2}}{\left(z-z_{j}\right)^{2}}+\frac{b_{-1}}{\left(z-z_{j}\right)}+\mathcal{O}\left(\left(z-z_{j}\right)^{0}\right) .
$$

The analytic function of interest when using BCFW recursion, $R(z) / z$, has the form 


$$
\frac{R(z)}{z}=\frac{b_{-2}}{z\left(z-z_{j}\right)^{2}}+\frac{b_{-1}}{z\left(z-z_{j}\right)}+\mathcal{O}\left(\left(z-z_{j}\right)^{0}\right),
$$

which we can rewrite in terms of $\delta=z-z_{j}$,

$$
\frac{R(z)}{z}=\frac{b_{-2}}{\left(\delta+z_{j}\right) \delta^{2}}+\frac{b_{-1}}{\left(\delta+z_{j}\right) \delta}+\mathcal{O}\left(\delta^{0}\right)
$$

Near the pole, $\delta<<1$, hence we can expand close to the pole,

$$
\frac{R(z)}{z}=\frac{b_{-2}}{\delta^{2} z_{j}}+\frac{1}{\delta}\left(-\frac{b_{-2}}{z_{j}^{2}}+\frac{b_{-1}}{z_{j}}\right)+\mathcal{O}\left(\delta^{0}\right)
$$

Writing the Laurent series in this way, exposes the contribution of the double pole term to the residue. The residue of the rational piece is then calculated with a contour integration and Cauchy's residue theorem,

$$
\frac{1}{2 \pi i} \oint_{\gamma} d z \frac{R(z)}{z}=R(0)+\left.\operatorname{Res}\left[\frac{R(z)}{z}\right]\right|_{z_{j}} .
$$

The contour, $\gamma$ is taken to infinity and the contribution at infinity must vanish for the rational piece to be recovered without requiring boundary terms.

The residue being,

$$
\left.\operatorname{Res}\left[\frac{R(z)}{z}\right]\right|_{z_{j}}=-\frac{b_{-2}}{z_{j}^{2}}+\frac{b_{-1}}{z_{j}} .
$$

In practice we employ an asymptotically well behaved complex shift on the amplitude, obtaining spinorial expressions that depend on the shift parameter $z$. This is not an expression of the amplitude in terms of a Laurent series. It is an expression that is closely linked to the Laurent series from which we can determine the coefficients $b_{-2}$ and $b_{-1}$ and show their dependence on the value of the pole $z_{j}{ }^{1}$.

\footnotetext{
${ }^{1}$ This is an important point. To be a well defined Laurent series, the coefficients of the poles must not depend on the shift variable $z$. They do however depend on the pole in question and will therefore vary according to $z_{j}$.
} 


\section{REVIEW OF TECHNIQUES FOR CALCULATING SCATTERING AMPLITUDES}

Considering the practical application of this procedure, we obtain an expression of the form

$$
\frac{R(z)}{z}=\frac{c_{-2}(z)}{z\left(z-z_{j}\right)^{2}}+\frac{c_{-1}(z)}{z\left(z-z_{j}\right)}+\mathcal{O}\left(\left(z-z_{j}\right)^{0}\right)
$$

where the functions $c_{i}(z)$ are a product of spinors that depend on the shift parameter. In order to determine the rational piece in general, we use Cauchy's residue theorem and the general expression for the contribution of $n$-th order poles to the residue,

$$
\left.\operatorname{Res}\left[\frac{f(z)}{\left(z-z_{j}\right)^{n}}\right]\right|_{z_{j}}=\frac{1}{(m-1) !} \lim _{z \rightarrow z_{j}}\left(\frac{d^{(n-1)}}{d z^{(n-1)}} \frac{\left(z-z_{j}\right)^{n} f(z)}{\left(z-z_{j}\right)^{n}}\right),
$$

where $f(z)$ is a holomorphic function of spinorst and we have extracted the $n$-th order pole.

Consequently, the residue of 2.172 is

$$
\left.\operatorname{Res}\left[\frac{R(z)}{z}\right]\right|_{z_{j}}=-\frac{c_{-2}\left(z_{j}\right)}{z_{j}^{2}}+\frac{1}{z_{j}}\left(\left.\frac{\mathrm{d}}{\mathrm{d} z} c_{-2}(z)\right|_{z_{j}}+c_{-1}\left(z_{j}\right)\right) .
$$

Thus, we have identified the coefficients in (2.173) through the residues (2.177) and (2.178) as

$$
b_{-2}=c_{-2}\left(z_{j}\right) \quad b_{-1}=\left.\frac{\mathrm{d}}{\mathrm{d} z} c_{-2}(z)\right|_{z_{j}}+c_{-1}\left(z_{j}\right) .
$$

The identification of the Laurent series coefficients demonstrates the dependence on the location of the pole. That is to say, the coefficients are not universal and vary with regard to the specific momentum channel taken on-shell.

In subsection 2.10.5 we will see an explicit example of this in terms of the leading in colour two-loop five-gluon all-plus rational piece. We also show how the first term in (2.180) is related to factorisation theorems, implying the remaining subleading contributions are non-factorising.

\footnotetext{
${ }^{1}$ In the explicit double pole example, $f(z)$ would be $c_{-2}(z)$.
} 
So in principle, we may use Cauchy's residue theorem provided we know the value of $c_{-2}(z)$, the leading pole and $c_{-1}(z)$ the subleading pole coefficients.

\subsubsection{The Rational Part of Two-Loop All-Plus Ampli- tudes: Double Poles}

This technique is best illustrated in the context of a calculation. In the following sections we examine the factorisations that give double pole contributions from a two-loop all-plus amplitude in general at first, then specialising to $n=5$.

To perform the recursion we require $R(z) \rightarrow 0$ as $|z| \rightarrow \infty$ which we can achieve by exciting the amplitude using the Risager shift [37] (2.182), here we choose to shift the legs $a, b$ and $c$ 目

$$
\begin{aligned}
\lambda_{a} \rightarrow \hat{\lambda}_{a} & =\lambda_{a}+z[b c] \lambda_{\eta}, \\
\lambda_{b} \rightarrow \hat{\lambda}_{b} & =\lambda_{b}+z[c a] \lambda_{\eta}, \\
\lambda_{c} \rightarrow \hat{\lambda}_{c} & =\lambda_{c}+z[a b] \lambda_{\eta} .
\end{aligned}
$$

It follows that excited complex poles will be functions only of the $\lambda$ spinors. The shift also introduces a reference spinor $\lambda_{\eta}$ into the functional form of the rational piece. The rational piece is independent of the choice of $\lambda_{\eta}$ (since it is a reference spinor) and we may reconstruct the rational piece to generate a form that is explicitly independent of $\lambda_{\eta}$. This will be examined in section 3.7.

This shift will excite diagrams that contain poles in $z$. In general they will correspond to factorisation shown in (2.163). As we are focussing on double poles from two-loop amplitudes however, we will constrain the discussion to the one-loop to one-loop factorisations where the double poles reside.

General factorisation theorems only determine the value of the leading pole. As of yet there are no general theorems that allow us to construct the subleading pole structure using four-dimensional on-shell methods. Therefore we must deduce the value of the subleading pole on a case by case basis using augmented

\footnotetext{
${ }^{1}$ In principle, we can shift any combination of legs for the all-plus.
} 


\section{REVIEW OF TECHNIQUES FOR CALCULATING SCATTERING AMPLITUDES}

recursion. This approach has seen success at one-loop [81, 84, 85] and twoloops [86 89], the latter two references forming part of chapter 3 and chapter 4.

To analyse the double pole structure we use axial gauge methods. The advantage of axial gauge is that, although it is an off-shell method, helicity still labels the internal legs and the vertices can be expressed in terms of nullified momenta [122, 132] defined using,

$$
k^{b}=k-\frac{k^{2}}{[q|k| q\rangle} q,
$$

where $q$ is a null reference vector.

Double poles in two-loop (and one-loop) amplitudes arise in diagrams where there is a factorisation of the amplitude into a product of a one-loop single minus amplitude (or a non-vanishing tree-amplitude) and crucially the one-loop threepoint vertex.

In the axial gauge formalism the one-loop three-point all-plus vertex is expressed as,

$$
A_{3}^{(1)}\left(a^{+}, b^{+}, k^{+}\right)=\frac{V_{3}^{(1)}\left(a^{+}, b^{+}, k^{+}\right)}{k^{2}}=\frac{i}{3} \frac{[a b]\left[b k^{b}\right]\left[k^{b} a\right]}{k^{2}}
$$

which is only non-zero for complex momental.

Consider the leading $s_{a b}$ double pole in one- and two-loop all-plus amplitudes. It can be written in terms of factorisations of the type

$$
V_{3}^{(1)}\left(a^{+}, b^{+}, k^{+}\right) \frac{-1}{s_{a b}^{2}} A^{(L)}\left(-k^{-}, c^{+}, \ldots, n^{+}\right)
$$

where $L=0,1$. The factorisation is shown diagrammatically in 2.6, and we will only consider $L=1$ from now on.

\footnotetext{
${ }^{1}$ and where we can express the one-loop all-plus amplitude as a vertex function with the propagator extracted explicitly.
} 


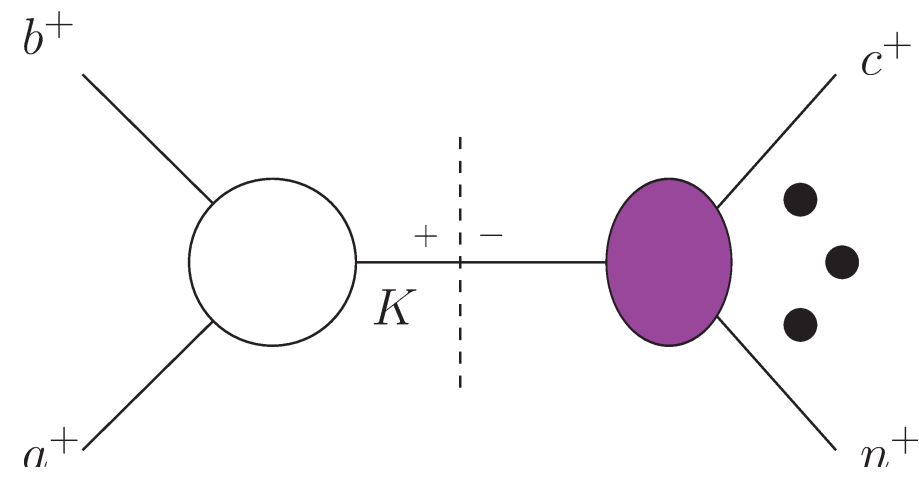

Figure 2.6: The origin of the double pole. The double pole corresponds to the coincidence of the singularity arising in the complex three-point all-plus amplitude and the propagator when the amplitude factorises as $K^{2}=s_{a b} \rightarrow 0$.

The full pole contribution 1 of eq.(2.175) arises from applying a Risager shift to the amplitude and taking residues of all excited diagrams of the form shown in fig. 2.7.

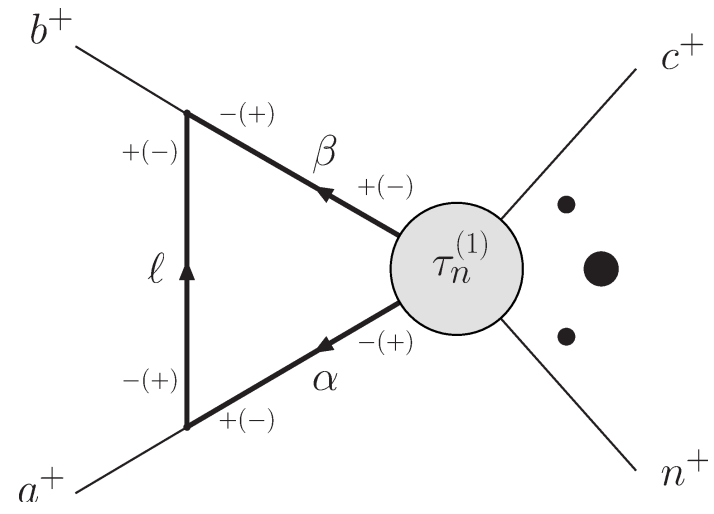

Figure 2.7: Diagram containing the leading and sub-leading poles as $s_{a b} \rightarrow 0$. The axial gauge construction permits the off-shell continuation of the internal legs. The two internal helicity configurations must be summed over to obtain the complete contribution to the $1 / s_{a b}$ residue. The one-loop current $\tau_{n}^{(1)}$ can be built from the on-shell $n$-point one-loop single minus amplitude.

This diagram can be expressed as a loop integral containing two off-shell tree-level amplitudes and a doubly off-shell current, $\tau_{n}^{(L)}$, which encodes the in-

\footnotetext{
${ }^{1}$ Including the non-factorising components.
} 


\section{REVIEW OF TECHNIQUES FOR CALCULATING SCATTERING AMPLITUDES}

formation that gives the double pole and subsequent subleading single pole contributions. There are two helicity configurations of the diagram and the full pole structure is only recovered by summing the two, as in (2.186).

Expressing the 3 -pt vertices in axial gauge, fig. 2.7 gives the integrall $\frac{i}{c_{\Gamma}(2 \pi)^{D}} \int \frac{d^{D} \ell}{\ell^{2} \alpha^{2} \beta^{2}} \frac{[a|\ell| q\rangle[b|\ell| q\rangle}{\langle a q\rangle\langle b q\rangle} \frac{\langle\beta q\rangle^{2}}{\langle\alpha q\rangle^{2}}\left[\tau_{n}^{(1)}\left(\alpha^{-}, \beta^{+}, c^{+}, \ldots, n^{+}\right)+\tau_{n}^{(1)}\left(\alpha^{+}, \beta^{-}, c^{+}, \ldots, n^{+}\right)\right]$.

The integral contributes one power of $s_{a b}^{-1}$ upon evaluation, and the doubly off-shell current contains terms that are $\mathcal{O}\left(s_{a b}^{-1}\right)$ and terms that are finite and/or polynomial in $s_{a b}$. Once the integration has been performed, it is the singular terms in the current that coincide with general factorisations. The remaining terms that are finite in $s_{a b}$ in the current acquire the factor of $s_{a b}^{-1}$ from the integration to give the non-factorising contributions. The current, $\tau_{n}^{(1)}$, is pivotal to augmented recursion.

If the current being considered is the leading in colour current, the second helicity configuration can be related to the first using the flip symmetry followed by the cycling and relabelling of the legs,

$$
\tau_{n: 1}^{(1)}\left(\alpha^{+}, \beta^{-}, c^{+}, \ldots, n^{+}\right)=(-1)^{n} \tau_{n: 1}^{(1)}\left(\beta^{-}, \alpha^{+}, n^{+}, \ldots, c^{+}\right)
$$

We are free to relabel the off-shell legs since they have been treated in the same manner, likewise with the external on-shell legs. This implies that only one current needs to be calculated, $\tau_{n}^{(1)}\left(\alpha^{-}, \beta^{+}, c^{+}, \ldots, n^{+}\right)$.

The pole as $s_{a b} \rightarrow 0$ arises from regions of the loop momentum integration where three adjacent propagators are simultaneously on-shell. The pole corresponds to a momentum configuration where $p_{a} \| p_{b}$, since $s_{a b}=0 \Longrightarrow 2 p_{a} \cdot p_{b}=0$.

\footnotetext{
${ }^{1}$ Where the factor $c_{\Gamma}$ is inserted in order to be consistent with the convention set out in (2.17).
} 


\subsection{Rational Contributions to Loop Amplitudes}

When $\ell^{2}=0, \alpha^{2}=0$ and $\beta^{2}=0$, we have

$$
\alpha^{2}=2 p_{a} \cdot \ell=0, \quad \beta^{2}=2 p_{b} \cdot \ell=0, \quad s_{a b}=s_{\alpha \beta}=2 \alpha \cdot \beta=0,
$$

such that all the internal legs are close to collinear.

Since we are really only interested in the function derived from fig. 2.7 in this particular collinear region, these relations can be used to simplify the current. Any powers of $\ell^{2}, \alpha^{2}$ or $\beta^{2}$ appearing in the numerator of the integrand generate powers of $s_{a b}$ when the loop momentum integration is performed, and can hence be regarded as small, allowing for expansions in $s_{a b}$. Similarly $\langle a \alpha\rangle,\langle a \beta\rangle,\langle b \alpha\rangle$ and $\langle b \beta\rangle$ can all be regarded as small.

Moreover, we will ultimately be taking residues of this integrated expression on the $s_{a b}$ pole. Any terms that are $\mathcal{O}\left(s_{a b}\right)$ or higher in the current can be ignored as they will vanish in the residue. Instead of generating the full off-shell current, it is sufficient for $\tau_{n}^{(1)}$ to satisfy two conditions [85]:

(C1) it reproduces the leading poles in $s_{\alpha \beta} \rightarrow 0$ shown in fig. 2.8.

(C2) in the limit $\alpha^{2}, \beta^{2} \rightarrow 0$ (the on-shell limit) the $n$-point one-loop single minus amplitude is reproduced.

a.

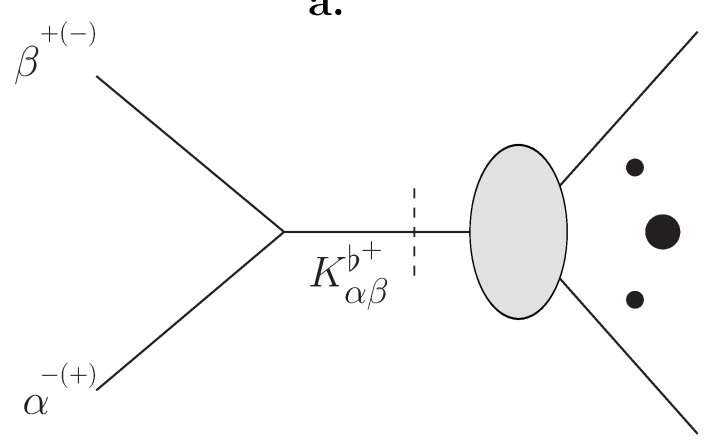

b.

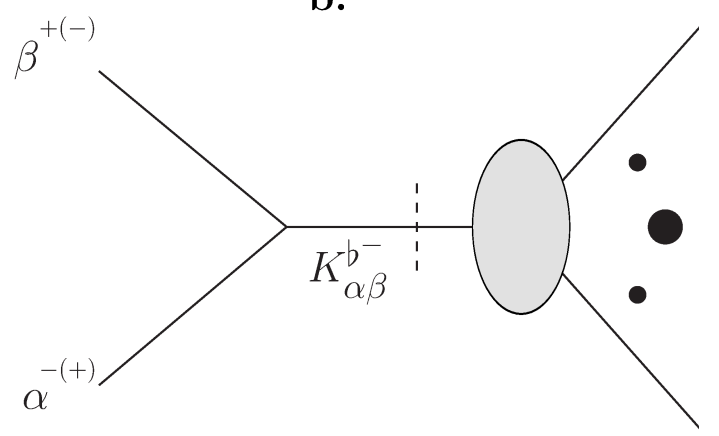

Figure 2.8: The factorisations that $\tau_{n}^{(1)}$ must reproduce.

Condition (C1) provides a starting point for constructing the current. In the next section, we show how to expose the non-factorising subleading pole 


\section{REVIEW OF TECHNIQUES FOR CALCULATING SCATTERING AMPLITUDES}

contribution by deriving the current needed for the rational part of the leading in colour two-loop five-point all-plus amplitude.

We reiterate here, that this off-shell meromorphic function, $\tau_{n}^{(1)}\left(\alpha^{-}, \beta^{+}, c^{+}, d^{+}, e^{+}\right)$ was deduced in [89] with the choice of shifted reference spinor only set to equal to the axial gauge reference spinor $\lambda_{\eta}=\lambda_{q}$. This differs from the function first found in [86] which set $\lambda_{\eta}=\lambda_{q}=\lambda_{d}$.

\subsubsection{Determining the leading current, $\tau_{5 ; 1}^{(1)}\left(\alpha^{-}, \beta^{+}, c^{+}, d^{+}, e^{+}\right)$.}

The leading adjacent current is 2 derived from the one-loop single-minus five-point amplitude [148],

$$
\begin{aligned}
A_{5 ; 1}^{(1)}\left(\alpha^{-}, \beta^{+}, c^{+}, d^{+}, e^{+}\right) & =\frac{i}{3} \frac{1}{\langle c d\rangle^{2}}\left(\frac{\langle c e\rangle\langle\alpha d\rangle^{3}[d e]}{\langle\alpha \beta\rangle\langle d e\rangle^{2}\langle\beta c\rangle}+\frac{\langle\alpha c\rangle^{3}\langle\beta d\rangle[\beta c]}{\langle d e\rangle\langle\alpha e\rangle\langle\beta c\rangle^{2}}-\frac{[\beta e]^{3}}{[\alpha \beta][e \alpha]}\right) \\
& =\sum_{j=i, i i, i i i} A_{5 ; 1 j}^{(1)}\left(\alpha^{-}, \beta^{+}, c^{+}, d^{+}, e^{+}\right)
\end{aligned}
$$

where

$$
\begin{aligned}
A_{5 ; 1 i}^{(1)}\left(\alpha^{-}, \beta^{+}, c^{+}, d^{+}, e^{+}\right) & =\frac{i}{3} \frac{1}{\langle c d\rangle^{2}}\left(\frac{\langle c e\rangle\langle\alpha d\rangle^{3}[d e]}{\langle\alpha \beta\rangle\langle d e\rangle^{2}\langle\beta c\rangle}\right), \\
A_{5 ; 1 i i}^{(1)}\left(\alpha^{-}, \beta^{+}, c^{+}, d^{+}, e^{+}\right) & =\frac{i}{3} \frac{1}{\langle c d\rangle^{2}}\left(-\frac{[\beta e]^{3}}{[\alpha \beta][e \alpha]}\right), \\
A_{5 ; 1 i i i}^{(1)}\left(\alpha^{-}, \beta^{+}, c^{+}, d^{+}, e^{+}\right) & =\frac{i}{3} \frac{1}{\langle c d\rangle^{2}}\left(\frac{\langle\alpha c\rangle^{3}\langle\beta d\rangle[\beta c]}{\langle d e\rangle\langle\alpha e\rangle\langle\beta c\rangle^{2}}\right) .
\end{aligned}
$$

We choose to separate the amplitude into a sum of terms for two reasons. Firstly, to identify the term that will ultimately become the double pole coefficient, $b_{-2}$ in the Laurent series (2.172) around $s_{a b}$. Secondly, to identify the terms that are associated to the factorisations of the current on the $s_{\alpha \beta}$ pole as shown in fig.2.8

\footnotetext{
${ }^{1}$ dubbed the adjacent current

${ }^{2}$ Creatively named as such since the off-shell legs are adjacent.
} 


\subsection{Rational Contributions to Loop Amplitudes}

and written in the axial gauge formalism as

$$
\tau_{5: 1 d p: \text { fact }}^{(1), \alpha^{-} \beta^{+}}=\frac{[\beta k]\langle\alpha q\rangle^{2}}{\langle\beta q\rangle\langle k q\rangle} \frac{1}{s_{\alpha \beta}} A_{4}^{(1)}\left(k^{-}, c^{+}, d^{+}, e^{+}\right)=\frac{i}{3} \frac{\langle\alpha q\rangle^{2}}{\langle\beta q\rangle^{2}} \frac{\langle e c\rangle\langle q|\alpha \beta| q\rangle}{s_{\alpha \beta}\langle c d\rangle\langle d e\rangle}\left(\frac{[c e]^{3}}{\left[e\left|k_{\alpha \beta}\right| q\right\rangle\left[c\left|k_{\alpha \beta}\right| q\right\rangle}\right),
$$

and

$$
\tau_{5: 1 \text { sb:fact }}^{(1), \alpha^{-} \beta^{+}}=\frac{\langle\alpha k\rangle[\beta q]^{2}}{[\alpha q][k q]} \frac{1}{s_{\alpha \beta}} A_{4}^{(1)}\left(k^{+}, c^{+}, d^{+}, e^{+}\right)=-\frac{i}{3} \frac{\langle\alpha k\rangle[\beta q]^{2}}{[\alpha q][k q]} \frac{1}{s_{\alpha \beta}} \frac{[e k]^{2}}{\langle c d\rangle^{2}} .
$$

We must expose these singularities explicitly since we are keeping the reference momenta general.

$A_{5 ; 1 i}^{(1)}$ is singular in $\langle\alpha \beta\rangle$ and will give rise to the double pole. It can be expressed as a sum of terms; the factorisation of the current on $s_{\alpha \beta}$ pole (2.193) and contributions to the subleading pole structure. $A_{5 ; 1 i i}^{(1)}$ is singular in $[\alpha \beta]$ and corresponds to the second helicity configuration in the factorisation of the current on the $s_{\alpha \beta}$ pole given by (2.194) and ultimately contributing to $b_{-1}$ in (2.172). Similarly, $A_{5 ; 1 i i i}^{(1)}$ is not singular in either $\langle\alpha \beta\rangle$ or $[\alpha \beta]$ and when integrated will contribute to the subleading pole structure, $b_{-1}$.

Turning our focus to $A_{5 ; 1 i}^{(1)}$, we apply the following formula to expose the $s_{\alpha \beta}$ pole by stripping off a term finite in $s_{\alpha \beta}$,

$$
\begin{aligned}
\frac{1}{\langle\alpha \beta\rangle\langle\beta c\rangle} & =\frac{[\beta \alpha]\left[q\left|k_{\alpha \beta}\right| c\right\rangle\langle\beta q\rangle}{s_{\alpha \beta}\langle\beta c\rangle\langle\beta q\rangle\left[q\left|k_{\alpha \beta}\right| c\right\rangle} \\
& =\frac{1}{\langle\alpha q\rangle\langle\beta q\rangle^{2}}\left(\frac{\langle q|\alpha \beta| q\rangle\left[q\left|k_{\alpha \beta}\right| q\right\rangle}{s_{\alpha \beta}\left[q\left|k_{\alpha \beta}\right| c\right\rangle}+\frac{\langle q \beta\rangle\langle q c\rangle[q|\alpha| q\rangle}{\langle\beta c\rangle\left[q\left|k_{\alpha \beta}\right| c\right\rangle}\right) .
\end{aligned}
$$

Using this, we rewrite $A_{5 ; 1 i}^{(1)}$ while factoring out $\langle\alpha q\rangle^{2} /\langle\beta q\rangle^{2}$ for ease of integration. Simultaneously, by capping on-shell instances of the legs we wish to continue off-shell, i.e. $\langle\alpha X\rangle$, with $[\beta \alpha]$ in both the numerator and denominator, 


\section{REVIEW OF TECHNIQUES FOR CALCULATING SCATTERING AMPLITUDES}

we can perform the off-shell continuation using,

$$
\frac{[\beta \alpha]\langle\alpha X\rangle}{[\beta \alpha]\langle\alpha q\rangle}=\frac{\left[\beta\left|k_{\alpha \beta}^{b}\right| X\right\rangle}{\left[\beta\left|k_{\alpha \beta}\right| q\right\rangle}=\frac{\left[q\left|k_{\alpha \beta}\right| X\right\rangle}{\left[q\left|k_{\alpha \beta}\right| q\right\rangle}+s_{\alpha \beta} \frac{[\beta|q| X\rangle}{\left[\beta\left|k_{\alpha \beta}\right| q\right\rangle\left[q\left|k_{\alpha \beta}\right| q\right\rangle}+\mathcal{O}\left(s_{\alpha \beta}^{2}\right) .
$$

Taking $k_{\alpha \beta}^{b}$, off-shell disregarding any $\mathcal{O}\left(s_{\alpha \beta}\right)$ terms that will vanish once the residue is taken and performing some on-shell simplifications,

$$
\begin{aligned}
A_{5 ; 1 i}^{(1)} & \left(\alpha^{-}, \beta^{+}, c^{+}, d^{+}, e^{+}\right)=\frac{i}{3} \frac{\langle c e\rangle[d e]}{\langle c d\rangle^{2}\langle d e\rangle^{2}} \frac{\langle\alpha q\rangle^{2}}{\langle\beta q\rangle^{2}} \frac{\left[\beta\left|k_{\alpha \beta}^{b}\right| d\right\rangle^{3}}{\left[\beta\left|k_{\alpha \beta}\right| q\right\rangle^{3}}\left(\frac{\langle q|\alpha \beta| q\rangle\left[q\left|k_{\alpha \beta}\right| q\right\rangle}{s_{\alpha \beta}\left[q\left|k_{\alpha \beta}\right| c\right\rangle}+\frac{\langle q \beta\rangle\langle q c\rangle[q|\alpha| q\rangle}{\langle\beta c\rangle\left[q\left|k_{\alpha \beta}\right| c\right\rangle}\right) \\
& =\frac{i}{3} \frac{\langle c e\rangle[d e]}{\langle c d\rangle^{2}\langle d e\rangle^{2}} \frac{\langle\alpha q\rangle^{2}}{\langle\beta q\rangle^{2}} \frac{\left[q\left|k_{\alpha \beta}\right| d\right\rangle^{3}}{\left[q\left|k_{\alpha \beta}\right| q\right\rangle^{2}} \frac{\langle q|\alpha \beta| q\rangle}{s_{\alpha \beta}\left[q\left|k_{\alpha \beta}\right| c\right\rangle} \\
& +\frac{i}{3} \frac{\langle c e\rangle[d e]}{\langle c d\rangle^{2}\langle d e\rangle^{2}} \frac{\langle\alpha q\rangle^{2}}{\langle\beta q\rangle^{2}}\left(-3 \frac{\langle q d\rangle[q|\beta| q\rangle\left[q\left|k_{\alpha \beta}\right| d\right\rangle^{2}}{\left[q\left|k_{\alpha \beta}\right| c\right\rangle\left[q\left|k_{\alpha \beta}\right| q\right\rangle^{2}}+\frac{\left[q\left|k_{\alpha \beta}\right| d\right\rangle^{3}}{\left[q\left|k_{\alpha \beta}\right| q\right\rangle^{3}} \frac{\langle q \beta\rangle\langle q c\rangle[q|\alpha| q\rangle}{\langle\beta c\rangle\left[q\left|k_{\alpha \beta}\right| c\right\rangle}\right) \\
& +\mathcal{O}\left(s_{\alpha \beta}\right) .
\end{aligned}
$$

The first term is close to the factorisation. Manipulating part of the first term in (2.198),

$$
\frac{\langle c e\rangle\left[q\left|k_{\alpha \beta}\right| d\right\rangle^{3}[d e]}{\left[q\left|k_{\alpha \beta}\right| q\right\rangle^{2}\left[q\left|k_{\alpha \beta}\right| c\right\rangle}=\frac{\langle e c\rangle\left[q\left|k_{\alpha \beta}\right| d\right\rangle^{2}[c e]}{\left[q\left|k_{\alpha \beta}\right| q\right\rangle^{2}}+\frac{\langle e c\rangle\left[q\left|k_{\alpha \beta}\right| d\right\rangle^{2}[q e] s_{\alpha \beta}}{\left[q\left|k_{\alpha \beta}\right| q\right\rangle^{2}\left[q\left|k_{\alpha \beta}\right| c\right\rangle}
$$

we extract another term contributing to $b_{-1}$. The final step to extract the factorisation (2.193) is the following expansion around $s_{\alpha \beta}$ 国. Consequently, we can eliminate the factors of $\left[q\left|k_{\alpha \beta}\right| d\right\rangle^{2}$ in the numerator of terms like,

$$
\begin{aligned}
& \frac{\left[q\left|k_{\alpha \beta}\right| d\right\rangle^{2}[c e]^{2}}{\langle c d\rangle\langle d e\rangle[c e]^{2}\left[q\left|k_{\alpha \beta}\right| q\right\rangle^{2}}=\frac{\left[q\left|k_{\alpha \beta}\right| d\right\rangle^{2}[c e]^{2}}{\left[e\left|k_{\alpha \beta}\right| d\right\rangle\left[c\left|k_{\alpha \beta}\right| d\right\rangle\left[q\left|k_{\alpha \beta}\right| q\right\rangle^{2}} \\
& =\frac{[c e]^{2}}{\left[e\left|k_{\alpha \beta}\right| q\right\rangle\left[c\left|k_{\alpha \beta}\right| q\right\rangle}\left(1+\frac{[c q]\langle q d\rangle s_{\alpha \beta}}{\left[c\left|k_{\alpha \beta}\right| q\right\rangle\left[q\left|k_{\alpha \beta}\right| d\right\rangle}\right)^{-1}\left(1+\frac{[e q]\langle q d\rangle s_{\alpha \beta}}{\left[e\left|k_{\alpha \beta}\right| q\right\rangle\left[q\left|k_{\alpha \beta}\right| d\right\rangle}\right)^{-1} \\
& =\frac{[c e]^{2}}{\left[e\left|k_{\alpha \beta}\right| q\right\rangle\left[c\left|k_{\alpha \beta}\right| q\right\rangle}\left(1-s_{\alpha \beta}\left(\frac{[c q]\langle q d\rangle}{\left[c\left|k_{\alpha \beta}\right| q\right\rangle\left[q\left|k_{\alpha \beta}\right| d\right\rangle}+\frac{[e q]\langle q d\rangle}{\left[e\left|k_{\alpha \beta}\right| q\right\rangle\left[q\left|k_{\alpha \beta}\right| d\right\rangle}\right)\right) \\
& +\mathcal{O}\left(s_{\alpha \beta}^{2}\right) .
\end{aligned}
$$

\footnotetext{
${ }^{1} s_{\alpha \beta}$ can be considered small since the pole arises in regions of the integration for which all three internal propagators are on shell.
} 


\subsection{Rational Contributions to Loop Amplitudes}

These manipulations applied concurrently give the following result with the factorisation of the current (2.193) explicitly exposed. The additional terms contribute to the non-factorising single pole contribution,

$$
\begin{aligned}
& A_{5 ; 1 i}^{(1)}\left(\alpha^{-}, \beta^{+}, c^{+}, d^{+}, e^{+}\right)=\frac{i}{3} \frac{\langle\alpha q\rangle^{2}}{\langle\beta q\rangle^{2}} \frac{\langle e c\rangle\langle q|\alpha \beta| q\rangle}{s_{\alpha \beta}\langle c d\rangle\langle d e\rangle}\left(\frac{[c e]^{3}}{\left[e\left|k_{\alpha \beta}\right| q\right\rangle\left[c\left|k_{\alpha \beta}\right| q\right\rangle}\right) \\
& \quad+\frac{i}{3} \frac{\langle\alpha q\rangle^{2}}{\langle\beta q\rangle^{2}} \frac{\langle e c\rangle[c e]^{2}\langle q|\alpha \beta| q\rangle}{\langle c d\rangle\langle d e\rangle\left[c\left|k_{\alpha \beta}\right| q\right\rangle\left[e\left|k_{\alpha \beta}\right| q\right\rangle}\left(\frac{[q e]}{\left[q\left|k_{\alpha \beta}\right| c\right\rangle}-\frac{[c e][c q]\langle q d\rangle}{\left[c\left|k_{\alpha \beta}\right| q\right\rangle\left[q\left|k_{\alpha \beta}\right| d\right\rangle}-\frac{[c e][e q]\langle q d\rangle}{\left[e\left|k_{\alpha \beta}\right| q\right\rangle\left[q\left|k_{\alpha \beta}\right| d\right\rangle}\right) \\
& \quad+\frac{i}{3} \frac{\langle c e\rangle[d e]}{\langle c d\rangle^{2}\langle d e\rangle^{2}} \frac{\langle\alpha q\rangle^{2}}{\langle\beta q\rangle^{2}}\left(-3 \frac{\langle q d\rangle[q|\beta| q\rangle\left[q\left|k_{\alpha \beta}\right| d\right\rangle^{2}}{\left[q\left|k_{\alpha \beta}\right| c\right\rangle\left[q\left|k_{\alpha \beta}\right| q\right\rangle^{2}}+\frac{\left[q\left|k_{\alpha \beta}\right| d\right\rangle^{3}}{\left[q\left|k_{\alpha \beta}\right| q\right\rangle^{3}} \frac{\langle q\rangle\langle q c\rangle[q|\alpha| q\rangle}{\langle\beta c\rangle\left[q\left|k_{\alpha \beta}\right| c\right\rangle}\right) \\
& \quad+\mathcal{O}\left(s_{\alpha \beta}\right) .
\end{aligned}
$$

At this stage $A_{5 ; 1 i}^{(1)}$ is almost well defined for $\alpha^{2}, \beta^{2} \neq 0$, the last term contains the ratio $\langle q \beta\rangle /\langle\beta c\rangle$ for which the denominator is has not been continued off-shell. We may use a result that is only well defined when considering the current as part of the diagram in fig.2.7, where the current has been integrated, and $\alpha=a+\ell$ and $\beta=b-l$. In this context we may use the Schouten identity,

$$
\frac{\langle X \alpha\rangle}{\langle Y \alpha\rangle}=\frac{\langle X \alpha\rangle}{\langle Y \alpha\rangle} \frac{\langle Y a\rangle}{\langle Y a\rangle}=\frac{\langle X a\rangle}{\langle Y a\rangle}+\mathcal{O}(\langle\alpha a\rangle),
$$

since factors of $\langle\alpha a\rangle,\langle\alpha b\rangle,\langle\beta a\rangle,\langle\beta b\rangle$ integrate to $\langle a b\rangle$ they can be considered small; additionally, they will not contribute to the residue.

Next we consider,

$$
A_{5 ; 1 i i}^{(1)}\left(\alpha^{-}, \beta^{+}, c^{+}, d^{+}, e^{+}\right)=\frac{i}{3} \frac{1}{\langle c d\rangle^{2}}\left(-\frac{[\beta e]^{3}}{[\alpha \beta][e \alpha]}\right) .
$$

This term is singular in $[\alpha \beta]$ and we may associate it to the factorisation (2.194) 


\section{REVIEW OF TECHNIQUES FOR CALCULATING SCATTERING AMPLITUDES}

by Schoutening terms in the numerator to expose the pole,

$$
\begin{aligned}
A_{5 ; 1 i i}^{(1)}\left(\alpha^{-}, \beta^{+}, c^{+}, d^{+}, e^{+}\right) & =-\frac{i}{3} \frac{1}{\langle c d\rangle^{2}} \frac{[\beta e]^{3}}{[\alpha \beta][e \alpha]} \\
& =-\frac{i}{3} \frac{1}{\langle c d\rangle^{2}} \frac{[\beta e]^{2}}{[\alpha \beta][e \alpha][\alpha q]}[\alpha q][\beta e] \\
& =-\frac{i}{3} \frac{1}{\langle c d\rangle^{2}} \frac{[\beta e]^{2}[q e]}{[e \alpha][\alpha q]}-\frac{i}{3} \frac{1}{\langle c d\rangle^{2}} \frac{[\beta e]^{2}[q \beta]}{[\alpha \beta][\alpha q]}
\end{aligned}
$$

We take the $[\beta e]^{2}$ term off-shell using,

$$
\frac{[\beta e]^{2}[\beta q]\langle\beta \alpha\rangle}{s_{\alpha \beta}[\alpha q]}=-\frac{\left\langle\alpha k^{b}\right\rangle[\beta q]^{2}\left[e k^{b}\right]^{2}}{s_{\alpha \beta}[\alpha q][k q]}+\frac{[e|q| \alpha\rangle}{[q|k| q\rangle[\alpha q][k q]}\left([e \beta][\beta q][k q]+[\beta q]^{2}\left[e k^{b}\right]\right) .
$$

Through this manipulation the factorisation is exposed with the addition of subleading terms,

$$
\begin{aligned}
& A_{5 ; 1 i i}^{(1)}\left(\alpha^{-}, \beta^{+}, c^{+}, d^{+}, e^{+}\right)=-\frac{i\left\langle\alpha k^{b}\right\rangle[\beta q]^{2}}{3} \frac{1}{s_{\alpha \beta}} \frac{\left[e k^{b}\right]^{2}}{\langle c d\rangle^{2}} \\
& \quad-\frac{i}{3} \frac{1}{\langle c d\rangle^{2}}\left(\frac{[\beta e]^{2}[q e]}{[e \alpha][\alpha q]}-\frac{[e|q| \alpha\rangle}{[q|k| q\rangle[\alpha q][k q]}\left([e \beta][\beta q][k q]+[\beta q]^{2}\left[e k^{b}\right]\right)\right) .
\end{aligned}
$$

Summarising the other two terms originating from the on-shell five-point amplitude,

$$
\begin{aligned}
A_{5 ; 1 i}^{(1)} & \left(\alpha^{-}, \beta^{+}, c^{+}, d^{+}, e^{+}\right)=\frac{i}{3} \frac{\langle\alpha q\rangle^{2}}{\langle\beta q\rangle^{2}} \frac{\langle e c\rangle\langle q|\alpha \beta| q\rangle}{s_{\alpha \beta}\langle c d\rangle\langle d e\rangle}\left(\frac{[c e]^{3}}{\left[e\left|k_{\alpha \beta}\right| q\right\rangle\left[c\left|k_{\alpha \beta}\right| q\right\rangle}\right) \\
& +\frac{i}{3} \frac{\langle\alpha q\rangle^{2}}{\langle\beta q\rangle^{2}} \frac{\langle e c\rangle[c e]^{2}\langle q|\alpha \beta| q\rangle}{\langle c d\rangle\langle d e\rangle\left[c\left|k_{\alpha \beta}\right| q\right\rangle\left[e\left|k_{\alpha \beta}\right| q\right\rangle}\left(\frac{[q e]}{\left[q\left|k_{\alpha \beta}\right| c\right\rangle}-\frac{[c e][c q]\langle q d\rangle}{\left[c\left|k_{\alpha \beta}\right| q\right\rangle\left[q\left|k_{\alpha \beta}\right| d\right\rangle}-\frac{[c e][e q]\langle q d\rangle}{\left[e\left|k_{\alpha \beta}\right| q\right\rangle\left[q\left|k_{\alpha \beta}\right| d\right\rangle}\right) \\
& +\frac{i}{3} \frac{\langle c e\rangle[d e]}{\langle c d\rangle^{2}\langle d e\rangle^{2}} \frac{\langle\alpha q\rangle^{2}}{\langle\beta q\rangle^{2}}\left(-3 \frac{\langle q d\rangle[q|\beta| q\rangle\left[q\left|k_{\alpha \beta}\right| d\right\rangle^{2}}{\left[q\left|k_{\alpha \beta}\right| c\right\rangle\left[q\left|k_{\alpha \beta}\right| q\right\rangle^{2}}+\frac{\left[q\left|k_{\alpha \beta}\right| d\right\rangle^{3}}{\left[q\left|k_{\alpha \beta}\right| q\right\rangle^{3}} \frac{\langle q\rangle\langle q c\rangle[q|\alpha| q\rangle}{\langle\beta c\rangle\left[q\left|k_{\alpha \beta}\right| c\right\rangle}\right) \\
& +\mathcal{O}\left(s_{\alpha \beta}\right),
\end{aligned}
$$


and

$$
A_{5 ; 1 i i i}^{(1)}\left(\alpha^{-}, \beta^{+}, c^{+}, d^{+}, e^{+}\right)=\frac{i}{3} \frac{1}{\langle c d\rangle^{2}}\left(\frac{\langle\alpha c\rangle^{3}\langle\beta d\rangle[\beta c]}{\langle d e\rangle\langle\alpha e\rangle\langle\beta c\rangle^{2}}\right)
$$

we choose to rewrite these functions in the conventions set out in [86, 89].

$$
\begin{aligned}
\tau_{5: 1 d p}^{(1), \alpha^{-} \beta^{+}} & =\frac{i}{3} \frac{\langle\alpha q\rangle^{2}}{\langle\beta q\rangle^{2}} \frac{\langle e c\rangle\langle q|\alpha \beta| q\rangle}{\langle c d\rangle\langle d e\rangle} \frac{1}{s_{\alpha \beta}}\left(\frac{[c e]^{3}}{\left[e\left|k_{\alpha \beta}\right| q\right\rangle\left[c\left|k_{\alpha \beta}\right| q\right\rangle}\right) \\
\tau_{5: 1 s f}^{(1), \alpha^{-} \beta^{+}} & =-\frac{i}{3} \frac{\left\langle\alpha k^{b}\right\rangle[\beta q]^{2}}{[\alpha q][k q]} \frac{1}{s_{\alpha \beta}} \frac{\left[e k^{b}\right]^{2}}{\langle c d\rangle^{2}}, \\
\tau_{5: 1 a p}^{(1), \alpha^{-} \beta^{+}} & =\frac{i}{3} \frac{\langle\alpha q\rangle^{2}}{\langle\beta q\rangle^{2}}\left[\frac { \langle e c \rangle [ c e ] ^ { 2 } \langle q | \alpha \beta | q \rangle } { \langle c d \rangle \langle d e \rangle [ c | k _ { \alpha \beta } | q \rangle [ e | k _ { \alpha \beta } | q \rangle } \left(\frac{[q e]}{\left[q\left|k_{\alpha \beta}\right| c\right\rangle}-\frac{[c e][c q]\langle q d\rangle}{\left[c\left|k_{\alpha \beta}\right| q\right\rangle\left[q\left|k_{\alpha \beta}\right| d\right\rangle}\right.\right. \\
& \left.-\frac{[c e][e q]\langle q d\rangle}{\left[e\left|k_{\alpha \beta}\right| q\right\rangle\left[q\left|k_{\alpha \beta}\right| d\right\rangle}\right) \\
& +\frac{\langle c e\rangle[d e]}{\langle c d\rangle^{2}\langle d e\rangle^{2}}\left(-3 \frac{\langle q d\rangle[q|\beta| q\rangle\left[q\left|k_{\alpha \beta}\right| d\right\rangle^{2}}{\left[q\left|k_{\alpha \beta}\right| c\right\rangle\left[q\left|k_{\alpha \beta}\right| q\right\rangle^{2}}+\frac{\left[q\left|k_{\alpha \beta}\right| d\right\rangle^{3}}{\left[q\left|k_{\alpha \beta}\right| q\right\rangle^{3}} \frac{\langle q \beta\rangle\langle q c\rangle[q|\alpha| q\rangle}{\langle\beta c\rangle\left[q\left|k_{\alpha \beta}\right| c\right\rangle}\right) \\
& \left.+\frac{1}{\langle c d\rangle^{2}} \frac{\langle\alpha c\rangle^{3}\langle\beta d\rangle[\beta c]}{\langle d e\rangle}\langle\alpha e\rangle\langle\beta c\rangle^{2}\right]+\mathcal{O}\left(s_{\alpha \beta}\right), \\
\tau_{5: 1 s l}^{(1), \alpha^{-} \beta^{+}} & =-\frac{i}{3} \frac{1}{\langle c d\rangle^{2}}\left(\frac{[\beta e]^{2}[q e]}{[e \alpha][\alpha q]}\right), \\
\tau_{5: 1 s k}^{(1), \alpha^{-} \beta^{+}} & =\frac{i}{3} \frac{1}{\langle c d\rangle^{2}}\left(\frac{[e|q| \alpha\rangle}{[q|k| q\rangle[\alpha q][k q]}\left([e \beta][\beta q][k q]+[\beta q]^{2}\left[e k^{b}\right]\right)\right)
\end{aligned}
$$

The leading current for this helicity configuration, $\tau_{5 ; 1}^{(1)}\left(\alpha^{-}, \beta^{+}, c^{+}, d^{+}, e^{+}\right)$is,

$$
\tau_{5 ; 1}^{(1)}\left(\alpha^{-}, \beta^{+}, c^{+}, d^{+}, e^{+}\right)=\tau_{5 ; 1 d p}^{(1), \alpha^{-} \beta^{+}}+\tau_{5 ; 1 s f}^{(1), \alpha^{-} \beta^{+}}+\tau_{5 ; 1 a p}^{(1), \alpha^{-} \beta^{+}}+\tau_{5 ; 1 s l}^{(1), \alpha^{-} \beta^{+}}+\tau_{5 ; 1 s k}^{(1), \alpha^{-} \beta^{+}}+\mathcal{O}\left(s_{\alpha \beta}\right) .
$$

We now move on to integrate this current. 


\section{REVIEW OF TECHNIQUES FOR CALCULATING SCATTERING AMPLITUDES}

\subsubsection{Integrating the leading current, $\tau_{5 ; 1}^{(1)}\left(\alpha^{-}, \beta^{+}, c^{+}, d^{+}, e^{+}\right)$.}

In order to obtain the rational term arising from the configuration in fig. 2.6 for $n=5$ the current must be integrated into the triangle,

$$
\int d \Lambda_{0}\left(\alpha^{+}, a^{+}, b^{+}, \beta^{-}\right) \tau_{5: 1}^{(1)}\left(\alpha^{-}, \beta^{+}, c^{+}, d^{+}, e^{+}\right)
$$

where the measure of the leading in colour contribution to the rational piece is defined as,

$$
\int d \Lambda_{0}\left(\alpha^{+}, a^{+}, b^{+}, \beta^{-}\right) \equiv \frac{i}{c_{\Gamma}(2 \pi)^{D}} \int \frac{d^{D} \ell}{\ell^{2} \alpha^{2} \beta^{2}} \frac{[a|\ell| q\rangle[b|\ell| q\rangle}{\langle a q\rangle\langle b q\rangle} \frac{\langle\beta q\rangle^{2}}{\langle\alpha q\rangle^{2}} \equiv \int d \Lambda_{0} .
$$

Integrating the double pole piece,

$$
\begin{aligned}
C_{d p}^{\alpha^{-} \beta^{+}} & =\frac{i}{3} \int d \Lambda_{0} \frac{\langle\alpha q\rangle^{2}}{\langle\beta q\rangle^{2}} \frac{\langle e c\rangle\langle q|\alpha \beta| q\rangle}{s_{\alpha \beta}\langle c d\rangle\langle d e\rangle}\left(\frac{[c e]^{3}}{\left[e\left|k_{\alpha \beta}\right| q\right\rangle\left[c\left|k_{\alpha \beta}\right| q\right\rangle}\right) \\
& =\frac{i}{18} \frac{\langle e c\rangle\langle q|b a| q\rangle}{s_{a b}\langle c d\rangle\langle d e\rangle} \frac{[a b]}{\langle a b\rangle}\left(\frac{[c e]^{3}}{\left[e\left|k_{a b}\right| q\right\rangle\left[c\left|k_{a b}\right| q\right\rangle}\right) \\
& =\frac{i}{18} \frac{\langle e c\rangle\langle q|a b| q\rangle}{\langle c d\rangle\langle d e\rangle\langle a b\rangle^{2}}\left(\frac{[c e]^{3}}{\left[e\left|k_{a b}\right| q\right\rangle\left[c\left|k_{a b}\right| q\right\rangle}\right),
\end{aligned}
$$

where we required the integral,

$$
\int d \Lambda_{0}[\langle q|\alpha \beta| q\rangle]=-\frac{1}{6} \frac{[a b]}{\langle a b\rangle}\langle q|a b| q\rangle+\mathcal{O}(\epsilon) .
$$

$C_{d p}^{\alpha^{+} \beta^{-}}$, the other helicity configuration of the current, has the same contribution and combining the two gives,

$$
C_{d p}=C_{d p}^{\alpha^{-} \beta^{+}}+C_{d p}^{\alpha^{+} \beta^{-}}=\frac{i}{9} \frac{\langle e c\rangle\langle q|a b| q\rangle}{\langle c d\rangle\langle d e\rangle\langle a b\rangle^{2}}\left(\frac{[c e]^{3}}{\left[e\left|k_{a b}\right| q\right\rangle\left[c\left|k_{a b}\right| q\right\rangle}\right)
$$




\subsection{Rational Contributions to Loop Amplitudes}

The first subleading pole contribution integral is,

$$
\begin{aligned}
& C_{a p}^{\alpha^{-} \beta^{+}}=\frac{i}{3} \int d \Lambda_{0} \\
& \times \frac{\langle\alpha q\rangle^{2}}{\langle\beta q\rangle^{2}}\left[\frac{\langle e c\rangle[c e]^{2}\langle q|\alpha \beta| q\rangle}{\langle c d\rangle\langle d e\rangle\left[c\left|k_{\alpha \beta}\right| q\right\rangle\left[e\left|k_{\alpha \beta}\right| q\right\rangle}\left(\frac{[q e]}{\left[q\left|k_{\alpha \beta}\right| c\right\rangle}-\frac{[c e][c q]\langle q d\rangle}{\left[c\left|k_{\alpha \beta}\right| q\right\rangle\left[q\left|k_{\alpha \beta}\right| d\right\rangle}-\frac{[c e][e q]\langle q d\rangle}{\left[e\left|k_{\alpha \beta}\right| q\right\rangle\left[q\left|k_{\alpha \beta}\right| d\right\rangle}\right)\right. \\
&+\frac{\langle c e\rangle[d e]}{\langle c d\rangle^{2}\langle d e\rangle^{2}}\left(-3 \frac{\langle q d\rangle[q|\beta| q\rangle\left[q\left|k_{\alpha \beta}\right| d\right\rangle^{2}}{\left[q\left|k_{\alpha \beta}\right| c\right\rangle\left[q\left|k_{\alpha \beta}\right| q\right\rangle^{2}}+\frac{\left[q\left|k_{\alpha \beta}\right| d\right\rangle^{3}}{\left[q\left|k_{\alpha \beta}\right| q\right\rangle^{3}} \frac{\langle q a\rangle\langle q c\rangle[q|\alpha| q\rangle}{\langle a c\rangle\left[q\left|k_{\alpha \beta}\right| c\right\rangle}\right) \\
&\left.+\frac{1}{\langle c d\rangle^{2}} \frac{\langle a c\rangle[c|\beta| d\rangle}{\langle d e\rangle\langle e a\rangle}\right]+\mathcal{O}(\langle a \beta\rangle),
\end{aligned}
$$

where we have used the approximation (2.202) to simplify the integration, discarding terms of order $\langle a \beta\rangle,\langle b \beta\rangle,\langle a \alpha\rangle,\langle b \alpha\rangle$ that will not contribute to the residue after integration. After using (2.218), the integrated terms are,

$$
\begin{aligned}
C_{a p}^{\alpha^{-\beta^{+}}} & =-\frac{i}{18} \frac{[a b]}{\langle a b\rangle}\left[\frac{\langle e c\rangle[c e]^{2}\langle q|b a| q\rangle}{\langle c d\rangle\langle d e\rangle\left[c\left|k_{a b}\right| q\right\rangle\left[e\left|k_{a b}\right| q\right\rangle}\left(\frac{[q e]}{\left[q\left|k_{a b}\right| c\right\rangle}-\frac{[c e][c q]\langle q d\rangle}{\left[c\left|k_{a b}\right| q\right\rangle\left[q\left|k_{a b}\right| d\right\rangle}-\frac{[c e][e q]\langle q d\rangle}{\left[e\left|k_{a b}\right| q\right\rangle\left[q\left|k_{a b}\right| d\right\rangle}\right)\right. \\
& +\frac{\langle c e\rangle[d e]}{\langle c d\rangle^{2}\langle d e\rangle^{2}}\left(-3 \frac{\langle q d\rangle[q|d+2 e| q\rangle\left[q\left|k_{a b}\right| d\right\rangle^{2}}{\left[q\left|k_{a b}\right| c\right\rangle\left[q\left|k_{a b}\right| q\right\rangle^{2}}+\frac{\left[q\left|k_{a b}\right| d\right\rangle^{3}}{\left\langle q\left|k_{a b}\right| q\right\rangle^{3}} \frac{\langle q a\langle q c\rangle[q|2 d+e| q\rangle}{\langle a c\rangle\left[q\left|k_{a b}\right| c\right\rangle}\right) \\
& \left.+\frac{1}{\langle c d\rangle^{2}} \frac{\langle a c\rangle[c|d+2 e| d\rangle}{\langle d e\rangle\langle e a\rangle}\right]+\mathcal{O}(\langle a b\rangle) .
\end{aligned}
$$

The third piece is related to the other helicity configuration of fig. 2.6 and we can make use of the factorisation to write the integral in terms of splitting functions. If we write,

$$
\begin{aligned}
C_{s f}^{\alpha^{-} \beta^{+}} & =\int d \Lambda_{0} \frac{\langle\alpha k\rangle[\beta q]^{2}}{[\alpha q][k q]} \frac{1}{s_{\alpha \beta}} A^{(1)}\left(k^{+}, c^{+}, d^{+}, e^{+}\right) \\
& =C_{t r i}^{\alpha^{-} \beta^{+}} \times \frac{1}{s_{\alpha \beta}} A^{(1)}\left(k^{+}, c^{+}, d^{+}, e^{+}\right)
\end{aligned}
$$

then the triangle integral

$$
C_{t r i}^{\alpha^{-} \beta^{+}}=\int \frac{d^{D} \ell}{\ell^{2} \alpha^{2} \beta^{2}} \frac{[a|\ell| q\rangle[b|\ell| q\rangle}{\langle a q\rangle\langle b q\rangle} \frac{\langle\beta q\rangle^{2}}{\langle\alpha q\rangle^{2}} \frac{\langle\alpha k\rangle[\beta q]^{2}}{[\alpha q][k q]},
$$




\section{REVIEW OF TECHNIQUES FOR CALCULATING SCATTERING AMPLITUDES}

is closely related to the $(+,+,-)$ one-loop splitting function such that we find

$$
C_{s f}^{\alpha^{-\beta^{+}}}+C_{s f}^{\alpha^{+} \beta^{-}}=\frac{1}{3} \frac{[q a][q b][b a]}{[k q]^{2}} \frac{1}{s_{a b}} A^{(1)}\left(k^{+}, c^{+}, d^{+}, e^{+}\right) .
$$

The fourth piece is

$$
C_{s l}^{\alpha^{-\beta^{+}}}=\frac{i}{3} \int d \Lambda_{0} \frac{1}{\langle c d\rangle^{2}}\left(-\frac{[\beta e]^{2}[q e]}{[e \alpha][\alpha q]}\right)
$$

and after extracting a factor of $\langle\beta q\rangle^{2} /\langle\alpha q\rangle^{2}$ from the measure and rewriting, we have,

$$
C_{s l}^{\alpha^{-} \beta^{+}}=\frac{i}{3} \int d \Lambda_{0}^{\prime} \frac{[q e]}{\langle c d\rangle^{2}}\left(-\frac{\left[e\left|k_{a b}\right| q\right\rangle^{2}}{[e|\alpha| q\rangle[q|\alpha| q\rangle}+2 \frac{\left[e\left|k_{a b}\right| q\right\rangle}{[q|\alpha| q\rangle}-\frac{[e|\alpha| q\rangle}{[q|\alpha| q\rangle}\right) .
$$

We promote $[q|\alpha| q\rangle$ in the denominator to a propagator, discarding terms of order $\alpha^{2}$ through,

$$
\frac{1}{2 \alpha \cdot q}-\frac{1}{(\alpha+q)^{2}}=\frac{\alpha^{2}}{2 \alpha \cdot q(\alpha+q)^{2}} .
$$

We also make use of the following to reduce box integrals to triangles using

$$
[a|\ell| q\rangle[b|\ell| q\rangle=\frac{\alpha^{2}\langle q|b \ell| q\rangle+\beta^{2}\langle q|a \ell| q\rangle+\ell^{2}\left\langle q\left|P_{a b} \beta\right| q\right\rangle}{\langle a b\rangle}
$$

Taking into account the promoted propagator, the only non-vanishing triangle is,

$$
-\frac{i}{3} \frac{[q e]}{\langle a b\rangle\langle c d\rangle^{2} 2 k . q} \int \frac{d^{D} \ell}{(2 \pi)^{D}} \frac{\langle q|b \ell| q\rangle[e|\alpha| q\rangle}{\langle a q\rangle\langle b q\rangle \ell^{2} \beta^{2}(\alpha+q)^{2}} .
$$

After Feynman parametrisation, only the scalar parts of the integrals survive since each loop momenta is capped with $\lambda_{q}$ and the integral evaluates to the rational result,

$$
C_{s l}^{\alpha^{-} \beta^{+}}=-\frac{i}{6} \frac{[q e][a b][e|b| q\rangle}{\langle a b\rangle\langle c d\rangle^{2} s_{b q}}
$$


Finally we have the $C_{s k}^{-+}$piece

$$
C_{s k}^{\alpha^{-} \beta^{+}}=\frac{i}{3} \int d \Lambda_{0} \frac{1}{\langle c d\rangle^{2}}\left(\frac{[e|q| \alpha\rangle}{[q|k| q\rangle[\alpha q][k q]}\left([e \beta][\beta q][k q]+[\beta q]^{2}\left[e k^{b}\right]\right)\right)
$$

which also makes use of (2.202) and (2.228) to simplify the integral, giving,

$$
\begin{aligned}
& C_{s k}^{\alpha^{-} \beta^{+}}=\frac{i}{3} \int \frac{d^{D} \ell}{(2 \pi)^{D}} \frac{[a|\ell| q\rangle[b|\ell| q\rangle}{\langle a q\rangle\langle b q\rangle \ell^{2} \alpha^{2} \beta^{2}} \frac{\langle\beta q\rangle^{2}}{\langle\alpha q\rangle^{2}} \\
& \times \frac{1}{\langle c d\rangle^{2}}\left(\frac{[e|q| \alpha\rangle}{[q|k| q\rangle[\alpha q][k q]}\left([e \beta][\beta q][k q]+[\beta q]^{2}\left[e k^{b}\right]\right)\right), \\
& C_{s k}^{\alpha^{-\beta^{+}}}=\frac{i}{18} \frac{[b a]}{\langle a b\rangle} \frac{[e q]}{\langle c d\rangle^{2} 2 k . q}\left(\frac{1}{s_{b q}}(5[q|b| q\rangle[e|b| q\rangle+3[q|a| q\rangle[e|b| q\rangle+[q|b| q\rangle[e|a| q\rangle)\right. \\
& \left.+\frac{\left[e\left|P_{a b}\right| q\right\rangle}{2 k \cdot q}(5[q|b| q\rangle+4[q|a| q\rangle)\right) .
\end{aligned}
$$

The full current is the sum of the above terms with the currents arising from the second helicity configuration of the current, $\tau_{5: 1}^{(1)}\left(\alpha^{+}, \beta^{-}, c^{+}, d^{+}, e^{+}\right)$that are obtained via (2.187). This can be done before integration, but is often more convenient to obtain this contribution post-integration. With this is mind (2.187) translates to replacing the off-shell legs with the on-shell external legs they are related to, here that is $\alpha \rightarrow a$ and $\beta \rightarrow b$.

\subsubsection{Calculating Residues}

The previous two sections have dealt with constructing a diagram that captures the full singularity structure of the Laurent series about the double pole contained in the two-loop five-point all-plus amplitude. We have determined the leading 


\section{REVIEW OF TECHNIQUES FOR CALCULATING SCATTERING AMPLITUDES}
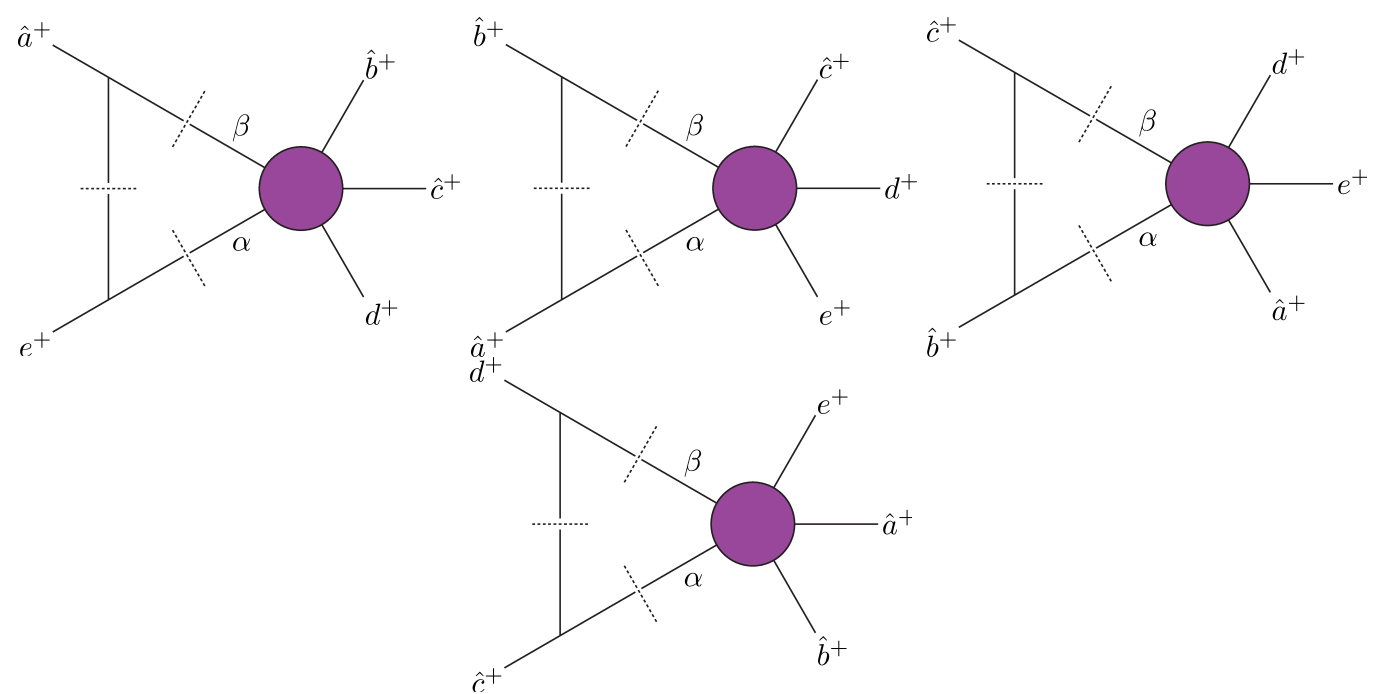

Figure 2.9: When the amplitude is excited with a Risager shift acting on the legs $p_{a}, p_{b}$ and $p_{c}$ with general $\lambda_{q}$, four diagrams corresponding to the rational part of the two-loop all-plus are excited.

double pole, consistent with factorisation theorems, and we have uncovered its subleading single pole. Having only had one eye on using complex recursion to generate the contribution of fig.2.7 to the rational part of the two-loop amplitude, we turn briefly to examine it and relate our findings to the residue shown in (2.172).

Performing the Risager shift on the external legs $p_{a}, p_{b}$ and $p_{c}$ excites poles in exactly four diagrams, each of which is a constituent of the full residue. One such diagram has the external leg configuration we have used up to this point and it is straightforward to obtain the remaining three diagrams by a simple cycling of legs. The four diagrams are shown in fig. 2.9 where the first set describe the two legs that are contained in the one-loop three-point amplitude when the amplitude factorises on the pole.

Taking the double pole term from the first diagram and showing the Risager shifted legs,

$$
\hat{C}_{d p}\left(\hat{a}^{+}, \hat{b}^{+}, \hat{c}^{+}, d^{+}, e^{+}\right)=\frac{i}{9} \frac{\langle e \hat{c}\rangle\langle q|a b| q\rangle}{\langle\hat{c} d\rangle\langle d e\rangle\langle\hat{a} \hat{b}\rangle^{2}}\left(\frac{[c e]^{3}}{\left[e\left|k_{a b}\right| q\right\rangle\left[c\left|k_{a b}\right| q\right\rangle}\right),
$$

we see that only the holomorphic spinors are shifted. Any spinors contracted 


\subsection{Rational Contributions to Loop Amplitudes}

with $\lambda_{q}$ are unshifted due to the fact that the shift reference spinor has been associated to the axial gauge reference spinor, $\lambda_{q}=\lambda_{\eta}$. This term is the first term in (2.178).

The double pole in the complex shift variable $z$ is

$$
\frac{1}{\langle\hat{a} \hat{b}\rangle^{2}}=\frac{1}{\left(\langle a b\rangle+z\left[c\left|k_{a b}\right| q\right\rangle\right)^{2}}=\frac{1}{\left[c\left|k_{a b}\right| q\right\rangle^{2}} \frac{1}{\left(z-z_{j}\right)^{2}},
$$

where the location of the pole is $z_{j}=-\frac{\langle a b\rangle}{\left[c\left|k_{a b}\right| q\right\rangle}$.

The contribution to the residue of the rational piece is calculated using (2.179) for $m=2$, resulting in

$$
\begin{aligned}
\left.\operatorname{Res}\left[\frac{c_{-2}(z)}{z\left(z-z_{j}\right)^{2}}\right]\right|_{z=z_{j}}= & \frac{i}{9}\left(-\frac{\langle q|a b| q\rangle[c e]^{3}\langle\hat{c} e\rangle}{\langle a b\rangle^{2}\langle\hat{c} d\rangle\langle d e\rangle\left[c\left|k_{a b}\right| q\right\rangle\left[e\left|k_{a b}\right| q\right\rangle}\right. \\
& \left.+\frac{\langle q|a b| q\rangle[c e]^{3}\langle\hat{c} e\rangle}{\langle a b\rangle^{2}\langle\hat{c} d\rangle\langle d e\rangle\left[c\left|k_{a b}\right| q\right\rangle^{2}\left[e\left|k_{a b}\right| q\right\rangle}\left(\frac{[a b]\langle c q\rangle}{\langle\hat{c} e\rangle}-\frac{[a b]\langle q d\rangle}{\langle\hat{c} d\rangle}\right)\right)\left.\right|_{z=z_{j}} .
\end{aligned}
$$

Therefore, the contribution of the double pole to the residue for this configuration is

$$
b_{2}=c_{-2}\left(z_{j}\right)=\frac{i}{9} \frac{\langle e \hat{c}\rangle\langle q|a b| q\rangle}{\langle\hat{c} d\rangle\langle d e\rangle\left[c\left|k_{a b}\right| q\right\rangle^{2}}\left(\frac{[c e]^{3}}{\left[e\left|k_{a b}\right| q\right\rangle\left[c\left|k_{a b}\right| q\right\rangle}\right) .
$$

The second term in (2.236) contributes to the non-factorising terms described by the coefficient $c_{-1}\left(z_{j}\right)$.

The remaining contributions to $c_{-1}\left(z_{j}\right)$ appear in the residues calculated from the functions

$$
\left.\operatorname{Res}\left[\frac{c_{-1}(z)}{z\left(z-z_{j}\right)}\right]\right|_{z=z_{j}}=\left.\operatorname{Res}\left[\frac{\hat{C}^{a p}+\hat{C}^{s f}+\hat{C}^{s l}+\hat{C}^{s k}}{z\left(z-z_{j}\right)}\right]\right|_{z=z_{j}}
$$

where the single pole structure has been explicitly shown for each term and the hats denote that all instances of the shifted spinors depend on $z$.

Combining the residue for this external leg configuration with the other three 


\section{REVIEW OF TECHNIQUES FOR CALCULATING}

SCATTERING AMPLITUDES

diagrams in Figure 2.9, gives the double pole - and its related subleading single pole - contribution to the rational part of the two-loop five-point diagram. We will see this specific calculation in the context of the full colour two-loop fivepoint all-plus amplitude in chapter 3, however we see the extension of this to sixand seven-point leading in colour gluon amplitudes in the following chapter. 


\section{Chapter 3}

\section{Analytic all-plus-helicity gluon amplitudes in QCD}

\subsection{Introduction}

The beginning of the 21st century saw the first leaps forward in the calculation of the scattering elements at next-to-next-to leading order (NNLO) for gluon scattering and the past twenty years have seen a great deal of progress. The accuracy of a cross-section calculation is dictated by NLO and higher order processes. As the sensitivity of detectors improves at particle colliders, in particular the LHC, we are required as theorists to hold up our end of the bargain and improve our theoretical estimates for cross-sections involving multiple processes including jets. At NNLO many helicity amplitudes involving quarks and gluons are still required. The foray into the realm of two-loop gluon amplitudes began with the calculation of the first full colour four-point gluon helicity amplitude the all-plus - in 2000 [70] using a unitarity based approach. The interference of the two-loop four-point amplitude with tree amplitudes radiating gluons relevant for NNLO along with its finite remainder being calculated soon after [93] via an alternative Feynman diagram approach. This was succeeded by the calculation of all the four-point helicity amplitudes contributing at two-loops in QCD [92] laying a solid foundation for higher multiplicity helicity amplitudes in the future, whilst also highlighting the increased complexity of integral functions that we could expect to meet. 


\section{ANALYTIC ALL-PLUS-HELICITY GLUON AMPLITUDES IN QCD}

The methods at four-point illustrated that it was possible to deduce these amplitudes analytically, however one could expect that the story at two-loop was not going to proceed as straightforwardly as it did at one-loop.

At the one-loop level, Passarino-Veltman reduction [52] gives a basis of integrals needed for the calculation of gluon amplitudes - box, triangle and bubble integrals, leaving behind a rational function to be determined. At two-loop however, one obtains thousands of Feynman diagrams and although a basis of integrals can be found on a case by case basis using differential equations [54, 97, 149, 150 and IBP relations [151] there is no method available to find an integral basis for a generic two-loop amplitude. Moreover, even when an ad hoc basis is found, often the next bottleneck is in the computation of the Feynman integrals that are present. These issues present considerable problems in the field and a great deal of time and effort has gone into both veins of research [152 159].

The two-loop five-point leading in colour amplitude was the first step toward higher multiplicity in gluon scattering calculations [94]. In this paper the authors developed a method of $D$-dimensional integrand reduction solving for a basis of master integrals, generalising the result of Ossola, Papadopoulos and Pittau [67]. Coupling this with generalised unitarity in $D$-dimensions to solve for the integrals coefficients, the branch cut structure and finite remainder was found. Although the majority of the result was known analytically, three of the integrals present in the final form depended on five scales and were unknown in analytic form, requiring numerical evaluation.

These methods at leading in colour saw an extension to full colour utilising treelevel BCJ relations to relate planar integrals to the non-planar integrals that arise in the amplitude at subleading in colour 95 .

By 2015, Henn et al. presented a compact form for the leading in colour twoloop five-point all-plus helicity amplitude [96] using differential equations [97]. This paper motivated an organisation of the unrenormalised amplitude into an IR divergent part and a finite remainder up to $\mathcal{O}(\epsilon)$. The remarkably compact form of the amplitude derived in both papers [94, 96 suggested perhaps this simplicity could be better illustrated via different means. The calculation of the two-loop five-point all-plus using the four-dimensional generalised unitarity and augmented recursion [86] showed such simplifications and set the starting point of 
a vein of work that lead to the work presented in this chapter. Four-dimensional unitarity shows us that the basis of integrals for the two-loop all-plus is in fact the same as the one-loop integral basis with one small caveat; the cuts of the one-loop all-plus amplitude are vanishing and force it to act as a vertex in these calculations. This drastically simplifies the amount of diagrams needed to deduce the cut constructible part of the amplitude. The trade-off being that the rational part of the amplitude must be calculated via other means, since the four-dimensional unitarity cuts aren't sensitive to rational terms. Augmented recursion was developed for this reason by the same authors, and had been successfully applied in many one-loop calculations in gravity and supergravity contexts [81, 84, 85] for loop amplitudes containing double poles. The combination of four-dimensional unitarity and augmented recursion then generated the first leading in colour sixpoint gluon scattering amplitude at two-loops [87. This simplified approach to calculating the cut constructible parts was subsequently used to deduce an all- $n$ result in Yang-Mills for the polylogarithmic part of the two-loop all-plus [111].

In this chapter, we present a detailed look at the work performed in [88] that lead to the leading in colour two-loop seven-gluon all-plus amplitude. We briefly review the one-loop functions that come into play in the four-dimensional unitarity methods. These functions are recycled in the approach in order to calculate the two-loop amplitude.

We will then follow the logic of ref. [111] and review the calculation of the $n$-point polylogarithmic terms generated by the cuts, as this is a general property of the two-loop all-plus and illustrates the simplicity of the cut constructible parts of the amplitude.

The largest contribution to the field from this work is in understanding the form of the seven-gluon all-plus rational piece. This amplitude contains double poles and augmented recursion must be applied in this case. We review the role of augmented recursion in the context of the six-gluon amplitude, since this result was rederived in the process of computing the seven-point, resulting in a simpler expression. The method used to rederive the six-point is identical to the method used for the seven-point, but the six-point is a more tractable example. 


\section{ANALYTIC ALL-PLUS-HELICITY GLUON AMPLITUDES IN QCD}

Performing a Risager shift] [37] on the amplitude excites factorisations containing the double pole i.e. the one-loop to one-loop factorisations, and also two-loop to tree factorisations that only contain single poles. The linchpin of augmented recursion is the use of axial gauge techniques [122] to obtain a doubly off-shell current that encodes the double pole structure of the rational piece, along with its vital subleading pole information, which we present at both six- and sevenpoint. Combining traditional BCFW [36] recursion techniques to capture the single pole structure and augmented recursion for the double pole structure, we derive the rational terms of the six- and seven-gluon amplitudes. This method initially builds a rational piece that is dependent on the reference spinor $\lambda_{\eta}$ that we set to the axial gauge reference spinor ${ }^{2} \lambda_{q}$. However, we present the result which is explicitly independent of the reference spinors and has the correct cyclic symmetric, by rebuilding the rational piece from the leading double pole and multiparticle pole factorisations.

\subsection{The all-plus helicity amplitude}

Before delving into the methods used to calculate the two-loop all-plus amplitude at six-point and seven-point, we take the time to review some general aspects of the leading in colour all-plus amplitude in general. The all-plus helicity amplitude at leading colour can be written as,

$$
\begin{aligned}
\mathcal{A}_{n: 1}\left(1^{+}, 2^{+}, \ldots, n^{+}\right)= & g^{n-2} \sum_{L \geq 1}\left(g^{2} N_{c} c_{\Gamma}\right)^{L} \times \sum_{\sigma \in S_{n} / Z_{n}} \operatorname{Tr}\left(T^{a_{\sigma(1)}} T^{a_{\sigma(2)}} T^{a_{\sigma(3)}} \cdots T^{a_{\sigma(n)}}\right) \\
& \times A_{n: 1}^{(L)}\left(\sigma(1)^{+}, \sigma(2)^{+}, \ldots, \sigma(n)^{+}\right)
\end{aligned}
$$

where $N_{c}$ is the number of colours and the summation is over the set of non-cyclic permutations, $S_{n} / Z_{n}$. This is the specific trace structure belonging to leading in colour amplitudes at all-loops. At two-loop $(L=2)$, it is only one of many partial amplitudes present, but dominates in the limit of large $N_{c}$. For the purposes of specificity we restrict the detail to the leading in colour amplitude for now.

\footnotetext{
${ }^{1}$ which introduces a reference spinor $\lambda_{\eta}$

${ }^{2}$ To avoid the excitation of spurious poles in the recursion.
} 
The tree-level all-plus amplitude vanishes as a consequence of supersymmetric Ward identities [72, 73]. The first non-vanishing amplitude in the pertubative expansion of the all-plus, is the rational one-loop amplitude [77]. Expressed to $\mathcal{O}\left(\epsilon^{0}\right)$ the leading contribution is given by,

$$
A_{n: 1}^{(1)}\left(1^{+}, 2^{+}, \cdots, n^{+}\right)=-\frac{i}{3} \sum_{1 \leq k_{1}<k_{2}<k_{3}<k_{4} \leq n} \frac{\left\langle k_{1} k_{2}\right\rangle\left[k_{2} k_{3}\right]\left\langle k_{3} k_{4}\right\rangle\left[k_{4} k_{1}\right]}{\langle 12\rangle\langle 23\rangle \cdots\langle n 1\rangle}+O(\epsilon) .
$$

All $-\epsilon$ forms of the one-loop amplitudes are given in terms of higher dimensional scalar integrals and are presented in section 2.10. The all- $\epsilon$ forms of the one-loop all-plus amplitudes are relevant as they appear as the coefficients of the IR divergent part of the leading in colour amplitudes [97. When taking collinear limits of the two-loop all-plus, the all- $\epsilon$ form of the one-loop amplitudes are required such that the collinear limits match order by order in $\epsilon$.

The focus of this chapter is the determination of the two-loop partial amplitude $A_{n: 1}^{(2)}\left(1^{+}, 2^{+}, \ldots, n^{+}\right)$for $n=7$, with the calculation put in the context of the result rederived for $n=6$. The leading in colour partial amplitude shares the symmetry of the trace structure it multiplies, that is, it has full cyclic symmetry,

$$
A_{n: 1}^{(2)}\left(1^{+}, 2^{+}, \ldots, n^{+}\right)=A_{n: 1}^{(2)}\left(2^{+}, \cdots, n^{+}, 1^{+}\right)
$$

and a flip symmetry,

$$
A_{n: 1}^{(2)}\left(1^{+}, 2^{+}, \ldots, n^{+}\right)=(-1)^{n} A_{n: 1}^{(2)}\left(n^{+}, \ldots, 2^{+}, 1^{+}\right)
$$

The IR and UV behaviours of this amplitude have been determined in general from group theory grounds [33] and motivate a partition of the amplitude into terms containing singularities in the dimensional regulator and finite pieces [96]: $A_{n: 1}^{(2)}\left(1^{+}, 2^{+}, \ldots, n^{+}\right)=A_{n: 1}^{(1)}\left(1^{+}, 2^{+}, \ldots, n^{+}\right)\left[-\sum_{i=1}^{n} \frac{1}{\epsilon^{2}}\left(\frac{\mu^{2}}{-s_{i, i+1}}\right)^{\epsilon}+\frac{n \pi^{2}}{12}\right]+F_{n: 1}^{(2)}+\mathcal{O}(\epsilon)$.

In this equation $A_{n: 1}^{(1)}$ is the all- $\epsilon$ form of the one-loop amplitude. The finite 
remainder function $F_{n: 1}^{(2)}$ is separated into polylogarithmic terms 1 l $P_{n: 1}^{(2)}$, arising from the evaluation of the cuts and terms that are purely rational functions of the spinor variables, $R_{n: 1}^{(2)}$,

$$
F_{n: 1}^{(2)}=P_{n: 1}^{(2)}+R_{n: 1}^{(2)}
$$

For this helicity configuration the functions composing $P_{n: 1}^{(2)}$ are completely determined using four-dimensional cuts. The result for arbitrary numbers of gluons 111] and the IR singularity structure determined by [33] completely specify the form of the cut constructible terms for the all-plus helicity configuration at two-loop for any number of external legs. In the following section on unitarity, we take the time to review the work of [111] in which $P_{n: 1}^{(2)}$ was determined in general.

$R_{n: 1}^{(2)}$ is a purely rational function that does not contain any branch cut singularities, only poles and is therefore missed by the four-dimensional unitarity cuts. The result for $R_{6: 1}^{(2)}$ was first presented in [87]. This result was not explicitly free of spurious singularities and required the inclusion of a term that was designed to remove the spurious singularities. In computing the seven-point rational term we understood to a greater extent how the pole structures stratifies, and by grouping together like poles showed the cancellation of spurious poles explicitly. This motivated us to rederive the six-point rational function and we present the methodology of that calculation here for brevity since the seven-point rational piece follows straightforwardly. We then present the result for $R_{7: 1}^{(2)}$.

\subsection{Determining $P_{n: 1}^{(2)}$ and Confirming the IR Sin- gularity Structure}

In this section, we review the construction of the cut constructible terms of the amplitude contributing to the IR singular terms and $P_{n: 1}^{(2)}$. We are able to obtain these using four-dimensional unitarity methods. Noting that the one-loop amplitudes have no four dimensional cuts since the order $\epsilon^{0}$ expression is purely

\footnotetext{
${ }^{1}$ finite as $\epsilon \rightarrow 0$
} 


\subsection{Determining $P_{n: 1}^{(2)}$ and Confirming the IR Singularity Structure}

rational, by using cuts of the amplitude where the momenta lie in four dimensions, the one-loop all-plus amplitude appears as a rational vertex. Consequently the cuts of the two-loop amplitude manifest as cuts of one-loop integral functions which can be evaluated using one-loop unitarity techniques. The integral basis for one-loop amplitudes contains box, triangle and bubble integral functions [4952], and the integral basis for the two-loop all-plus is given by fig. 3.1 in which the one-loop all-plus amplitude appears as a vertex. For completeness we review the $n$-point calculation presented in 111 .
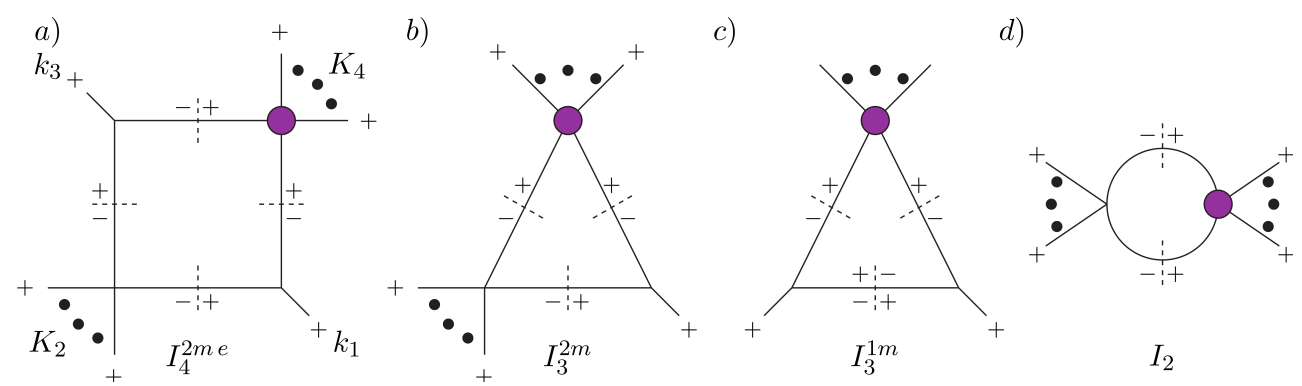

Figure 3.1: Four dimensional cuts of the two-loop all-plus amplitude involving an all-plus one-loop vertex. In the boxes $K_{2}$ may be null but $K_{4}$ must contain at least two external legs.

First consider the various box configurations. The only two non-vanishing contributions to the integral basis come from the configuration shown in fig. 3.2, including the one-mass box diagram present in the limit $K_{2} \rightarrow 0$. The three- and four-mass boxes have a vanishing contribution to the cuts since all external gluons have positive helicity. In the quadruple cut, massive corners will correspond to a contribution of a tree amplitude to the cut with four or more legs. As such at least two of these legs must have negative helicities in order to have a non-trivial cut. This requires each of the two internal cut legs to have negative helicity, but a negative helicity of an internal leg on one side of the cut corresponds to a positive helicity on the other side of the cut. Therefore, for boxes with three or more massive corners, there is no choice of helicity configuration of the internal legs that result in a non-vanishing cut.

Additionally, the limiting case, when $K_{2}^{2} \rightarrow 0$ has three, three-point trees that enter the cuts. This configuration is only non vanishing when there are alternating MHV and $\overline{M H V}$ amplitudes, such that the complex momenta cut 


\section{ANALYTIC ALL-PLUS-HELICITY GLUON AMPLITUDES IN QCD}

solutions do not require an unphysical momentum configuration. The last case to consider is one for which the corner corresponding to the one-loop all-plus as a vertex is massless. Any configuration where this corner is massless results in a vanishing contribution. If all corners are massless, massless Googly trees are adjacent and the cut conditions result in a vanishing contribution. Consider the following cut 1 ,

$$
\int d \operatorname{LIPS} A_{3: 1}^{(1)}\left(1^{+}, l_{2}^{+},-l_{1}^{+}\right) A_{3}^{(0)}\left(2^{+}, l_{3}^{+},-l_{2}^{-}\right) A_{3}^{(0)}\left(3^{+}, l_{4}^{+},-l_{3}^{-}\right) A_{3}^{(0)}\left(4^{+}, l_{1}^{-},-l_{4}^{-}\right) .
$$

Taking the corners involving $p_{1}$ and $p_{2}$, the cut conditions imply,

$$
\lambda_{1} \propto \lambda_{l_{2}}, \quad \lambda_{2} \propto \lambda_{l_{2}} \Longrightarrow \lambda_{1} \propto \lambda_{2} \Longrightarrow s_{12}=0
$$

which is not allowed in general. The same applies if there is one-massive tree corner. If there are two or more massive trees, again there are insufficient internal negative helicities to provide a non-vanishing result.

Therefore the only contributions to the boxes arise from fig. 3.2.

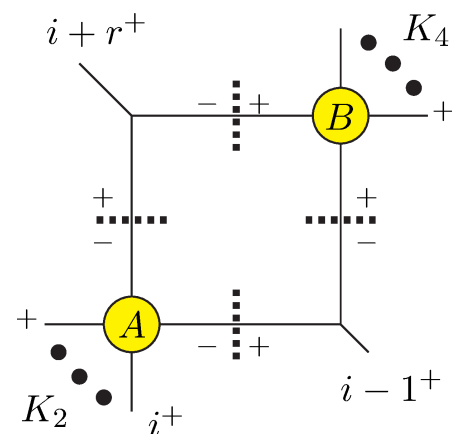

Figure 3.2: The non-vanishing quadruple cut. $A$ is a MHV tree amplitude whereas $B$ is a one-loop all-plus amplitude. The set $K_{2}$ may consist of a single leg but the set $K_{4}$ must contain at least two legs. The integral function depends upon $S \equiv\left(p_{i-1}+K_{2}\right)^{2}$ and $T \equiv\left(K_{2}+p_{i+r}\right)^{2}$.

The quadruple cuts [30] of fig. 3.2 generate the general box contributions to

\footnotetext{
${ }^{1}$ where there are two helicity configurations that give identical contributions
} 


\subsection{Determining $P_{n: 1}^{(2)}$ and Confirming the IR Singularity Structure}

the IR terms and $P_{n: 1}^{(2)}$. The cut is given by

$$
\begin{aligned}
\int d \operatorname{LIPS} A_{n-r: 1}^{(1)} & \left(-l_{1}^{+},(i+r+1)^{+}, \ldots,(i-2)^{+}, l_{2}^{+}\right) A_{3}^{(0)}\left(-l_{2}^{-},(i-1)^{+}, l_{3}^{+}\right) \\
& \times A_{r+2}^{(0)}\left(-l_{3}^{-}, i^{+}, \ldots,(i+r-1)^{+}, l_{4}^{-}\right) A_{3}^{(0)}\left(-l_{4}^{+},(i+r)^{+}, l_{1}^{-}\right)
\end{aligned}
$$

where the legs are taken to be $\bmod n$. As shown in [111], the coefficient of the one-loop box integral function $I_{4}^{2 m}\left(S, T, K_{2}^{2}, K_{4}^{2}\right)$ is given by

$$
\begin{aligned}
& \frac{\left[i-1\left|K_{4}\right| i+r\right\rangle\left[i+r\left|K_{4}\right| i-1\right\rangle}{\langle 12\rangle\langle 23\rangle\langle 34\rangle \cdots\langle n 1\rangle} \\
& \quad \times\left(\sum_{a<b<c<d \in K_{4}} \operatorname{tr}_{-}[a b c d]-\sum_{a<b<c \in K_{4}} \operatorname{tr}_{-}\left[a b c K_{4}\right]+\sum_{a<b \in K_{4}} \frac{\left\langle i-1\left|K_{4} a b K_{4}\right| i+r\right\rangle}{\langle i-1 i+r\rangle}\right),
\end{aligned}
$$

where $K_{4}$ is the sum of the momenta in the set $\{i+r+1, \cdots, i-2\}$ with a cyclic definition of indices, the inequality refers to ordering within the set. Additionally, the numerator of the coefficient satisfies, $\left[i-1\left|K_{4}\right| i+r\right\rangle\left[i+r\left|K_{4}\right| i-1\right\rangle=S T-$ $K_{2}^{2} K_{4}^{2}$ which cancels the coefficient of the two-mass easy box integral function. The corresponding integral function can be rewritten in the following way, by rearranging,

$$
\begin{aligned}
-2\left(S T-K_{2}^{2} K_{4}^{2}\right) I_{4}^{2 m}\left(S, T, K_{2}^{2}, K_{4}^{2}\right)= & \left(-\frac{\left(-S / \mu^{2}\right)^{-\epsilon}}{\epsilon^{2}}-\frac{\left(-T / \mu^{2}\right)^{-\epsilon}}{\epsilon^{2}}+\frac{\left(-K_{2}^{2} / \mu^{2}\right)^{-\epsilon}}{\epsilon^{2}}+\frac{\left(-K_{4}^{2} / \mu^{2}\right)^{-\epsilon}}{\epsilon^{2}}\right) \\
& +F^{2 m}\left[S, T, K_{2}^{2}, K_{4}^{2}\right]
\end{aligned}
$$

where the finite polylogarithmic terms arising in the two-mass box are written as

$$
\begin{aligned}
F^{2 m}\left[S, T, K_{2}^{2}, K_{4}^{2}\right] & =\operatorname{Li}_{2}\left[1-\frac{K_{2}^{2}}{S}\right]+\operatorname{Li}_{2}\left[1-\frac{K_{2}^{2}}{T}\right]+\operatorname{Li}_{2}\left[1-\frac{K_{4}^{2}}{S}\right] \\
& +\operatorname{Li}_{2}\left[1-\frac{K_{4}^{2}}{T}\right]-\operatorname{Li}_{2}\left[1-\frac{K_{2}^{2} K_{4}^{2}}{S T}\right]+\log ^{2}(S / T) / 2 .
\end{aligned}
$$

The divergent terms in (3.12) contribute to the full IR piece. The finite polylogarithmic part of the amplitude only has contributions from the remaining terms 


\section{ANALYTIC ALL-PLUS-HELICITY GLUON AMPLITUDES IN QCD}

in (3.12) since polylogarithms only arise in the one- and two-mass boxes present in the basis. Therefore, $P_{n: 1}^{(2)}$ is obtained by summing over all possible box contributions, including the degenerate cases when $K_{2}$ corresponds to a single leg $\left(K_{4}\right.$ must contain to at least two external legs).

For the sake of a compact expression, we briefly redefine the Mandlestam invariants as in refs. [53, 56],

$$
t_{i}^{[r]}=\left(p_{i}+\ldots p_{i+r-1}\right)^{2}
$$

such that the finite polylogarithms naturally take a form that is easily summed over,

$$
F_{n: r, i}^{2 m}=F^{2 m}\left[t_{i-1}^{[r+1]}, t_{i}^{[r+1]}, t_{i}^{[r]}, t_{i+r+1}^{[n-r-2]}\right]
$$

In this language, $S=t_{i-1}^{[r+1]}, T=t_{i}^{[r+1]}, K_{2}^{2}=t_{i}^{[r]}, K_{4}^{2}=t_{i+r+1}^{[n-r-2]}$, and $r=1$ is the case when $K_{2}^{2} \rightarrow 0$ and $F^{2 m}$ goes smoothly to $F^{1 m}$,

$F^{2 m}\left[S, T, 0, K_{4}^{2}\right]=F^{1 m}\left[S, T, K_{4}^{2}\right]=\operatorname{Li}_{2}\left[1-\frac{K_{4}^{2}}{S}\right]+\operatorname{Li}_{2}\left[1-\frac{K_{4}^{2}}{T}\right]+\log ^{2}(S / T) / 2+\frac{\pi^{2}}{6}$.

The full expression for $P_{n: 1}^{(2)}$ is [11],

$$
P_{n: 1}^{(2)}=-\frac{i}{3\langle 12\rangle\langle 23\rangle\langle 34\rangle \cdots\langle n 1\rangle} \sum_{i=1}^{n} \sum_{r=1}^{n-4} c_{r, i} F_{n: r, i}^{2 m}
$$

where the labels on the integral function correspond to those in fig. 4.2 and the coefficient is

$c_{r, i}=\left(\sum_{a<b<c<d \in K_{4}} \operatorname{Tr}_{-}[a b c d]-\sum_{a<b<c \in K_{4}} \operatorname{Tr}_{-}\left[a b c K_{4}\right]+\sum_{a<b \in K_{4}} \frac{\left\langle i-1\left|K_{4} a b K_{4}\right| i+r\right\rangle}{\langle i-1 i+r\rangle}\right)$.

There are also contributions from one- and two- mass triangle integral functions. Again, we are restricted to configurations for which there are no adjacent massive trees in the triple cuts as the identical helicity external legs require the 


\subsection{Determining $P_{n: 1}^{(2)}$ and Confirming the IR Singularity Structure}

introduction of a vanishing tree in these cases. There are two cases for which the one-loop all-plus corner is massless. The first case is the one for which there are two other massless corners or one massive corner. This case is akin to the box integral case shown with the relations (3.8). Here, the cut constraints impose that the sum of a subset of external momenta vanish. The second case pertains to two massive corners and there are insufficient internal negative helicity legs to constitute a non-vanishing cut.

The coefficients of the triangle integral functions are found by taking triple cuts of the amplitude and the cut integrals evaluated using canonical forms [60] (alternatively one can use [59]). The singularity structure found from performing the triple cuts only contributes to the IR singularities. The coefficients of the one-mass and two-mass triangles are given in [111.

The last type of integral in the basis is that of the bubble integral functions. The only non-vanishing helicity configuration allowed by the double cuts of the bubbles involves the one-loop all-plus amplitude and an MHV tree-level amplitude. The n-point two-particle cut is given by

$b_{r, i}=\int \mathrm{dLIPS} \frac{\left\langle l_{1} l_{2}\right\rangle^{3}}{\left\langle l_{2}(r+1)\right\rangle\langle(r+1)(r+2)\rangle \cdots\left\langle n l_{1}\right\rangle} \sum_{1 \leq i<j<k<l \leq n} \frac{\langle i|j k l| i]}{\left\langle l_{2} l_{1}\right\rangle\left\langle l_{1} 1\right\rangle\langle 12\rangle \cdots\left\langle r l_{2}\right\rangle}$.

The denominator of the cut integral is $\mathcal{O}\left(l^{-2}\right)$, and there are three types of spinor combinations of the loop momenta arising from the sum in the numerator of the one-loop all-plus,

$$
\left\langle l_{2}\left|l_{1} X Y\right| l_{2}\right], \quad\left\langle l_{2}|X Y Z| l_{2}\right], \quad\langle W|X Y Z| W]
$$

where $W, X, Y, Z$ denote the appearance of external momenta. The first two types of terms are $\mathcal{O}\left(l^{1}\right)$ whereas the last type are $\mathcal{O}\left(l^{0}\right)$. This means that at best the two particle cuts are $\mathcal{O}\left(l^{-1}\right)$ and hence will have already been captured by the triangle and box contributions. The computation shows that the bubble integral function has a vanishing coefficient. This fact is consistent with the absence of terms proportional to $\epsilon^{-1}$ outlined in subsection 2.8.3 and agrees with [33. 


\section{ANALYTIC ALL-PLUS-HELICITY GLUON AMPLITUDES IN QCD}

To obtain the full IR singular structure, one must sum over all possible box and triangle configurations. This can be done post integration with one particular configuration of the external legs. Performing the sum and collecting the IR singular terms gives 111],

$$
\left.\left(\sum \mathcal{C}_{i} I_{4, i}^{2 \mathrm{~m}}+\sum \mathcal{C}_{i} I_{3, i}^{2 \mathrm{~m}}+\sum \mathcal{C}_{i} I_{3, i}^{1 \mathrm{~m}}\right)\right|_{I R}=A_{n: 1}^{(1), \epsilon^{0}}\left(1^{+}, 2^{+}, \cdots, n^{+}\right) \times-\sum_{i=1}^{n} \frac{1}{\epsilon^{2}}\left(\frac{\mu^{2}}{-s_{i, i+1}}\right)^{\epsilon}
$$

where $A_{n: 1}^{(1), \epsilon^{0}}\left(1^{+}, 2^{+}, \cdots, n^{+}\right)$is the order $\epsilon^{0}$ truncation of the one-loop amplitude. This matches the universal form found by Catani for the leading in colour allplus [33]. A key step is to promote the coefficient of these terms to the all- $\epsilon$ form of the one-loop amplitude. This ensures that the two-loop amplitude has the correct singular structure as confirmed in [97.

This completely specifies the cut constructible part of the two-loop all-plus $n$-point gluon amplitude and thus contains the seven-point as a special case. Four-dimensional unitarity highlights the simplicity of the all-plus at two-loops by showing that the branch cut singularity structure is of the same class as a general one-loop amplitude. With the branch cut singularity structure completely determined the final task is to ascertain the form of the pole structure.

\subsection{The Six-Point Rational Term, $R_{6: 1}^{(2)}$}

To determine the rational part of the leading in colour two-loop all-plus sixpoint amplitude we use augmented recursion to obtain its full pole structure. Although this chapter centres on the calculation of $A_{7}^{(2)}$ it is far more manageable to work with the rational part of the six-point amplitudel. The six-point contains multiparticle poles, double poles and single poles in a similar way to the sevenpoint. Consequently, the methods applied here are easily extendible to the sevenpoint whilst being comprised of more tractable expressions. We present the full

\footnotetext{
${ }^{1}$ The six-point rational terms were calculated in [87] however the derivation of this six-point rational term is a new result in that it is explicitly free of spurious poles.
} 
derivation of the six-point rational term, presenting the results of the six- and seven-point rational terms at the end of the section.

Augmented recursion国, like other forms of complex recursion, employs the use of a complex shift to induce a dependence on a complex parameter $z$ in the amplitude. Both the BCFW and Risager shifts are complex shifts that break the symmetry of an amplitude by acting on specific legs. Furthermore, the Risager shift introduces a dependence on an arbitrary spinor $\lambda_{\eta}$ in the individual diagrams. Since this is an arbitrary reference spinor, the full sum of contributing diagrams should be independent of this momenta. While it is hard to determine a priori the large $z$ behaviour of an unknown amplitude, recovering cyclic symmetry (and $\lambda_{\eta}$ independence) are powerful checks.

For the two-loop all-plus amplitude this symmetry recovery does not occur for the BCFW shift (the one-loop all-plus amplitude has the same feature). At five-point this can be seen by applying the shift to the known result, while at six- and seven-point it can be seen retrospectively by applying the shift to the results we obtain. However, the Risager shift does vanish at infinity for the fivepoint amplitude and results in rational terms with the correct symmetries and factorisations for both the six- and seven-point amplitudes. This self-consistency provides a stringent check of the result.

In this section, we examine the factorisation channels that are excited by the Risager shift and contribute to the residue. We then focus on a particular collinear factorisation channel that contains double poles and construct a doubly off-shell current that captures the subleading pole information adequately. Having found a function that is not explicitly $\lambda_{\eta}$ independent, we then reconstruct the amplitude from its factorisations capturing its full pole structure at the cost of a finite ambiguity, that we construct an ansatz for and determine using the original expression.

\footnotetext{
${ }^{1}$ Introduced in section 2.10, where we only dealt with factorisations of the two-loop all-plus that contain double poles.
} 


\subsection{Factorisations of $R_{6}^{(2)}$}

In order to recognise where we must apply augmented recursion we must discuss the factorisations of $R_{6: 1}^{(2)}$. Applying the Risager shift to three adjacent legs, $a$, $b$ and $c$ of $A_{6: 1}^{(2)}(a, b, c, d, e, f)$ motivates a factorisation of the two-loop amplitude into two classes, one-loop to one-loop factorisations and tree to two-loop factorisations. The complex shift breaks the cyclic symmetry of the rational terms and also introduces a dependence on a reference momentum $\eta=\lambda_{\eta} \bar{\lambda}_{\eta}$. By identifying $\lambda_{\eta}$ with the axial gauge reference spinor $\lambda_{q}$ we prevent the excitation of spurious poles $\mathbb{1}^{1}$ such as $\langle\hat{a} q\rangle \longrightarrow 0$. Note that $P_{6: 1}^{(2)}$ does not contain any unphysical poles and so the factorisations of $R_{6: 1}^{(2)}$ are the physical factorisations of the amplitude.

The simple pole contributions to $R_{6: 1}^{(2)}$ arise in the multiparticle one-loop to one-loop factorisations,

$$
\begin{aligned}
& \sum_{h= \pm} A_{4: 1}^{(1)}\left(e, f, \hat{a}, \hat{K}^{h}\right) \frac{-1}{K^{2}} A_{4: 1}^{(1)}\left(-\hat{K}^{-h}, \hat{b}, \hat{c}, d\right) \\
& \sum_{h= \pm} A_{4: 1}^{(1)}\left(f, \hat{a}, \hat{b}, \hat{K}^{h}\right) \frac{-1}{K^{2}} A_{4: 1}^{(1)}\left(-\hat{K}^{-h}, \hat{c}, d, e\right)
\end{aligned}
$$

and all the tree to two-loop factorisations,

$$
\begin{array}{r}
A_{3}^{(0)}\left(\hat{a}, \hat{b}, \hat{K}^{-}\right) \frac{-1}{K^{2}} R_{5: 1}^{(2)}\left(-\hat{K}^{+}, \hat{c}, d, e, f\right), \\
A_{3}^{(0)}\left(f, \hat{a}, \hat{K}^{-}\right) \frac{-1}{K^{2}} R_{5: 1}^{(2)}\left(-\hat{K}^{+}, \hat{b}, \hat{c}, d, e\right), \\
R_{5: 1}^{(2)}\left(-\hat{K}^{+}, e, f, \hat{a}, \hat{b}\right) \frac{-1}{K^{2}} A_{3}^{(0)}\left(\hat{c}, d, \hat{K}^{-}\right), \\
R_{5: 1}^{(2)}\left(-\hat{K}^{+}, d, e, f, \hat{a}\right) \frac{-1}{K^{2}} A_{3}^{(0)}\left(\hat{b}, \hat{c}, \hat{K}^{-}\right),
\end{array}
$$

where $R_{5: 1}^{(2)}$ refers to the rational part of the leading in colour two-loop five-gluon all-plus amplitude. These factorisations contain single poles, whose residues can simply be evaluated at the appropriate complex pole. The remaining factorisa-

\footnotetext{
${ }^{1}$ Note there is no need to specify $\bar{\lambda}_{\eta}$ since we are using complex momenta and the poles only relate to poles in the $\lambda_{i}$ spinors
} 
tions are the collinear one-loop to one-loop factorisations that involve the threepoint one-loop all-plus vertex:

$$
\begin{gathered}
A_{3: 1}^{(1)}\left(f^{+}, \hat{a}, \hat{K}^{\lambda}\right) \frac{-1}{K^{2}} A_{5: 1}^{(1)}\left(-\hat{K}^{-\lambda}, \hat{b}, \hat{c}, d, e\right), \\
A_{3: 1}^{(1)}\left(\hat{c}, d, \hat{K}^{\lambda}\right) \frac{-1}{K^{2}} A_{5: 1}^{(1)}\left(-\hat{K}^{-\lambda}, e, f, \hat{a}, \hat{b}\right), \\
A_{3: 1}^{(1)}\left(\hat{a}, \hat{b}, \hat{K}^{\lambda}\right) \frac{-1}{K^{2}} A_{5: 1}^{(1)}\left(-\hat{K}^{-\lambda}, \hat{c}, d, e, f\right), \\
A_{3: 1}^{(1)}\left(\hat{b}, \hat{c}, \hat{K}^{\lambda}\right) \frac{-1}{K^{2}} A_{5: 1}^{(1)}\left(-\hat{K}^{-\lambda}, d, e, f, \hat{a}\right) .
\end{gathered}
$$

Diagrammatically, these are represented by fig. 2.6 where there is an explicit pole from the highlighted propagator and a further pole from the complex three-point one-loop amplitude (2.184). These factorisations are the now familiar source of double poles in loop amplitudes. It is these four diagrams that we must obtain by using augmented recursion.

\subsection{The $s_{a b}$ pole in the Six-Point Rational Term}

In this section we determine the current required to describe the leading and sub-leading poles in $R_{6: 1}^{(2)}$ as $s_{a b} \rightarrow 0$. As discussed in section 2.10, the current must satisfy two conditions,

(C1) it reproduces the leading poles in $s_{\alpha \beta} \rightarrow 0$ shown in fig. 2.8.

(C2) in the limit $\alpha^{2}, \beta^{2} \rightarrow 0$ (the on-shell limit) the $n$-point one-loop single minus amplitude is reproduced.

Naturally, to calculate the six-point doubly off-shell current we begin with the on-shell expression for the one-loop six-point single-minus amplitude [38], rewriting it to identify $\alpha$ and $\beta$ as the two legs we wish to continue off-shell and 


\section{ANALYTIC ALL-PLUS-HELICITY GLUON AMPLITUDES IN} QCD

organising the amplitude by its poles in $\langle\alpha \beta\rangle$ and $[\alpha \beta]$ :

$$
\begin{aligned}
A_{6: 1}^{(1)}\left(\alpha^{-}, \beta^{+}, c^{+}, d^{+}, e^{+}, f^{+}\right) \\
=\frac{i}{3}\left[\frac{1}{\langle\alpha \beta\rangle\langle\beta c\rangle\langle d e\rangle^{2}}\left(\frac{\left[f\left|K_{\beta c}\right| \alpha\right\rangle^{3}}{t_{\alpha \beta c}\left[f\left|K_{\alpha \beta}\right| c\right\rangle}+\frac{\langle\alpha e\rangle^{3}[e f]\langle d f\rangle}{\langle c d\rangle\langle e f\rangle^{2}}-\frac{\langle\alpha d\rangle^{3}\langle c e\rangle\left[d\left|K_{\beta c}\right| \alpha\right\rangle}{\langle c d\rangle^{2}\langle e f\rangle\langle f \alpha\rangle}\right)\right. \\
\quad+\frac{[\beta f]^{3}}{[\alpha \beta][f \alpha] t_{c d e}}\left(\frac{[\beta c][c d]}{\langle d e\rangle\left[\beta\left|K_{c d}\right| e\right\rangle}-\frac{[d e][e f]}{\langle c d\rangle\left[f\left|K_{\alpha \beta}\right| c\right\rangle}+\frac{[c e]}{\langle c d\rangle\langle d e\rangle}\right) \\
\left.\quad+\frac{\left[\beta\left|K_{c d}\right| \alpha\right\rangle^{3}}{\langle c d\rangle^{2}\langle e f\rangle\langle f \alpha\rangle t_{\beta c d}\left[\beta\left|K_{c d}\right| e\right\rangle}-\frac{\langle\alpha c\rangle^{3}[\beta c]\langle\beta d\rangle}{\langle\beta c\rangle^{2}\langle c d\rangle^{2}\langle d e\rangle\langle e f\rangle\langle f \alpha\rangle}\right]
\end{aligned}
$$

First, separating the terms with an eye to determining the leading and subleading pole structures,

$$
A_{6: 1}^{(1)}\left(\alpha^{-}, \beta^{+}, c^{+}, d^{+}, e^{+}, f^{+}\right)=\sum_{j=i, i i, i i i} A_{6: 1 j}^{(1)}\left(\alpha^{-}, \beta^{+}, c^{+}, d^{+}, e^{+}, f^{+}\right)
$$

where,

$$
\begin{array}{r}
A_{6: 1 i}^{(1)}\left(\alpha^{-}, \beta^{+}, c^{+}, d^{+}, e^{+}, f^{+}\right)=\frac{i}{3} \frac{1}{\langle\alpha \beta\rangle\langle\beta c\rangle\langle d e\rangle^{2}}\left(\frac{\left[f\left|K_{\beta c}\right| \alpha\right\rangle^{3}}{t_{\alpha \beta c}\left[f\left|K_{\alpha \beta}\right| c\right\rangle}+\frac{\langle\alpha e\rangle^{3}[e f]\langle d f\rangle}{\langle c d\rangle\langle e f\rangle^{2}}\right. \\
\left.-\frac{\langle\alpha d\rangle^{3}\langle c e\rangle\left[d\left|K_{\beta c}\right| \alpha\right\rangle}{\langle c d\rangle^{2}\langle e f\rangle\langle f \alpha\rangle}\right),
\end{array}
$$

$A_{6: 1 i i}^{(1)}\left(\alpha^{-}, \beta^{+}, c^{+}, d^{+}, e^{+}, f^{+}\right)=\frac{i}{3} \frac{[\beta f]^{3}}{[\alpha \beta][f \alpha] t_{c d e}}\left(\frac{[\beta c][c d]}{\langle d e\rangle\left[\beta\left|K_{c d}\right| e\right\rangle}-\frac{[d e][e f]}{\langle c d\rangle\left[f\left|K_{\alpha \beta}\right| c\right\rangle}+\frac{[c e]}{\langle c d\rangle\langle d e\rangle}\right)$

$$
A_{6: 1 i i i}^{(1)}\left(\alpha^{-}, \beta^{+}, c^{+}, d^{+}, e^{+}, f^{+}\right)=\frac{i}{3} \frac{\left[\beta\left|K_{c d}\right| \alpha\right\rangle^{3}}{\langle c d\rangle^{2}\langle e f\rangle\langle f \alpha\rangle t_{\beta c d}\left[\beta\left|K_{c d}\right| e\right\rangle}-\frac{\langle\alpha c\rangle^{3}[\beta c]\langle\beta d\rangle}{\langle\beta c\rangle^{2}\langle c d\rangle^{2}\langle d e\rangle\langle e f\rangle\langle f \alpha\rangle} .
$$

We take the legs off-shell by initially replacing $\alpha$ and $\beta$ by their nullified form within axial gauge. We choose to compute the rational piece with this particular 


\subsection{The $s_{a b}$ pole in the Six-Point Rational Term}

helicity configuration of the off-shell legs since we can relate the result obtained to the other helicity configuration via a flip symmetry, cycling and a relabelling of legs,

$$
\tau_{6: 1}^{(1)}\left(\alpha^{+}, \beta^{-}, c^{+}, d^{+}, e^{+}, f^{+}\right)=-\tau_{6: 1}^{(1)}\left(\alpha^{-}, \beta^{+}, f^{+}, e^{+}, d^{+}, c^{+}\right) .
$$

In order to satisfy condition $(\mathrm{C} 1)$, we need to ensure that we recover the leading singularities of the six-point all-plus amplitude. The pole in $s_{\alpha \beta}$ can arise from two sources when working in complex momenta, when $\langle\alpha \beta\rangle \rightarrow 0$ and $[\alpha \beta] \rightarrow 0$. Since we know that the rational part of the amplitude factorises on the $s_{\alpha \beta}$ pole, this implies the current factorises on these poles in a similar manner. By considering the factorisation of the current using the axial gauge formalism a priori, we can match the terms that are singular in $\langle\alpha \beta\rangle$ and $[\alpha \beta]$ to the factorisations of the current. Therefore, we identify terms in the one-loop six-point single-minus amplitude that contain these poles, consider the factorisations of the current and rewrite the amplitude taking $\alpha$ and $\beta$ off-shell to exactly reproduce the factorisations. This leads us to organise the amplitude as

$$
A_{6: 1}^{(1)}\left(\alpha^{-}, \beta^{+}, c^{+}, d^{+}, e^{+}, f^{+}\right)=\tau_{6: 1 d p}^{(1), \alpha^{-} \beta^{+}}+\tau_{6: 1 s b}^{(1), \alpha^{-} \beta^{+}}+\tau_{6: 1 n f}^{(1), \alpha^{-} \beta^{+}}+\mathcal{O}\left(s_{\alpha \beta}\right),
$$

where we write $\mathcal{O}\left(s_{\alpha \beta}\right)$ to specify the fact these terms are required in order to satisfy condition (C2), however they are not essential to this discussion as they will vanish when we take the residues of the current to obtain the rational contribution from fig. 2.7. The full derivation of each term contributing to the six-point current is shown in Appendix C, however it is beneficial to identify the source of the relevant terms here. $A_{6: 1 i}^{(1)}$ will generate the double pole part of the current and gives one factorisation of the current $\tau_{6: 1 d p}^{(1), \alpha^{-} \beta^{+}}$, while $A_{6: 1 i i}^{(1)}$ generates the single pole structure and the second factorisation of the current $2, \tau_{6: 1 s b}^{(1), \alpha^{-} \beta^{+}}$. The remaining term, $A_{6: 1 i i i}^{(1)}$ contributes to the non-factorising single pole contributions of the current, along with the off-shell corrections applied to $A_{6: 1 i}^{(1)}$ and $A_{6: 1 i i}^{(1)}$.

$$
\begin{aligned}
& { }^{1} \text { as }\langle\alpha \beta\rangle \rightarrow 0 . \\
& { }^{2} \text { as }[\alpha \beta] \rightarrow 0 .
\end{aligned}
$$


With this organisation it is natural to writen,

$$
\frac{i}{c_{\Gamma}(2 \pi)^{D}} \int \frac{d^{D} \ell}{\ell^{2} \alpha^{2} \beta^{2}} \frac{[a|\ell| q\rangle[b|\ell| q\rangle}{\langle a q\rangle\langle b q\rangle} \frac{\langle\beta q\rangle^{2}}{\langle\alpha q\rangle^{2}} \tau_{6: 1}^{(1)}\left(\alpha^{-}, \beta^{+}, c^{+}, d^{+}, e^{+}, f^{+}\right)=C_{d p}^{\alpha^{-} \beta^{+}}+C_{s b}^{\alpha^{-} \beta^{+}}+C_{n f}^{\alpha^{-} \beta^{+}}+\mathcal{O}\left(s_{a b}\right)
$$

where the terms multiplying the current are the off-shell axial gauge three-point gluon amplitudes proportional to (2.78). Since we will calculate fig. 2.7 using only one helicity configuration of the current, we may write,

$$
\int d \Lambda_{0}\left(\alpha^{+}, a^{+}, b^{+}, \beta^{-}\right) \equiv \frac{i}{c_{\Gamma}(2 \pi)^{D}} \int \frac{d^{D} \ell}{\ell^{2} \alpha^{2} \beta^{2}} \frac{[a|\ell| q\rangle[b|\ell| q\rangle}{\langle a q\rangle\langle b q\rangle} \frac{\langle\beta q\rangle^{2}}{\langle\alpha q\rangle^{2}} \equiv \int d \Lambda_{0} .
$$

It is useful to extract the term $\langle\alpha q\rangle^{2} /\langle\beta q\rangle^{2}$ from the terms in the current as the inverse of this term is present in the integral measure $d \Lambda_{0}$ and the cancellation of such terms simplifies the integration. The double poles arise from

$$
\begin{gathered}
C_{d p}^{\alpha^{-} \beta^{+}} \equiv \frac{i}{3} \int d \Lambda_{0}\left[\frac { \langle \alpha q \rangle ^ { 2 } } { \langle \beta q \rangle ^ { 2 } } \frac { \langle q | \alpha \beta | q \rangle [ q | K _ { a b } | q \rangle } { s _ { a b } [ q | K _ { a b } | c \rangle \langle d e \rangle ^ { 2 } } \left(-\frac{[f c]^{3}}{t_{a b c}\left[f\left|K_{a b}\right| c\right\rangle} \frac{\left[q\left|K_{a b}\right| c\right\rangle^{3}}{\left[q\left|K_{a b}\right| q\right\rangle^{3}}\right.\right. \\
\left.\left.+\frac{[e f]\langle d f\rangle}{\langle c d\rangle\langle e f\rangle^{2}} \frac{\left[q\left|K_{a b}\right| e\right\rangle^{3}}{\left[q\left|K_{a b}\right| q\right\rangle^{3}}-\frac{\langle c e\rangle[d c]}{\langle c d\rangle^{2}\langle e f\rangle} \frac{\left[q\left|K_{a b}\right| d\right\rangle^{3}}{\left[q\left|K_{a b}\right| q\right\rangle^{3}} \frac{\left[q\left|K_{a b}\right| c\right\rangle}{\left[q\left|K_{a b}\right| f\right\rangle}\right)\right] .
\end{gathered}
$$

The integral corresponding to the square bracket pole in the current is,

$$
\begin{gathered}
C_{s b}^{\alpha^{-} \beta^{+}}=\frac{i}{3} \int d \Lambda_{0}\left[\frac { [ \beta q ] ^ { 2 } } { [ \alpha q ] ^ { 2 } } \frac { [ q | \alpha \beta | q ] } { [ q | K _ { a b } | q \rangle ^ { 2 } } \frac { 1 } { s _ { a b } } \frac { [ f | K _ { a b } | q \rangle ^ { 2 } } { t _ { c d e } } \left(\frac{\left\langle q\left|K_{a b}\right| c\right][c d]}{\langle d e\rangle\left\langle q\left|K_{a b} K_{c d}\right| e\right\rangle}\right.\right. \\
\left.\left.-\frac{[d e][e f]}{\langle c d\rangle[f|k| c\rangle}+\frac{[c e]}{\langle c d\rangle\langle d e\rangle}\right)\right] .
\end{gathered}
$$

The remaining function constitutes the subleading non-factorising parts:

$$
C_{n f}^{\alpha^{-} \beta^{+}}=\int d \Lambda_{0} \tau_{6: 1 n f}^{(1), \alpha^{-} \beta^{+}}+\mathcal{O}\left(s_{\alpha \beta}\right)
$$

\footnotetext{
${ }^{1}$ The factor $c_{\Gamma}$ is inserted to make the normalisation consistent with eq. 3.1 .
} 
where $\tau_{6: 1 n f}^{(1), \alpha^{-} \beta^{+}}$is the expression defined in (C.24).

These expressions are integrated to obtain the contribution from fig. 4.5 (up to terms that are finite as $\left.s_{a b} \rightarrow 0\right)$. The explicit integrations may be found in appendix C.1 and we only keep the $\mathcal{O}\left(\epsilon^{0}\right)$ terms, i.e. the rational contributions of the integrations. The double pole integrates to 1

$$
\begin{array}{r}
\left.C_{d p}^{\alpha^{-} \beta^{+}}\right|_{\mathbb{Q}}=-\frac{i}{18} \frac{[a b]}{\langle a b\rangle} \frac{\langle q|a b| q\rangle\left[q\left|K_{a b}\right| q\right\rangle}{s_{a b}\left[q\left|K_{a b}\right| c\right\rangle\langle d e\rangle^{2}}\left(-\frac{[f c]^{3}}{t_{a b c}\left[f\left|K_{a b}\right| c\right\rangle} \frac{\left[q\left|K_{a b}\right| c\right\rangle^{3}}{\left[q\left|K_{a b}\right| q\right\rangle^{3}}\right. \\
\left.+\frac{[e f]\langle d f\rangle}{\langle c d\rangle\langle e f\rangle^{2}} \frac{\left[q\left|K_{a b}\right| e\right\rangle^{3}}{\left[q\left|K_{a b}\right| q\right\rangle^{3}}-\frac{\langle c e\rangle[d c]}{\langle c d\rangle^{2}\langle e f\rangle} \frac{\left[q\left|K_{a b}\right| d\right\rangle^{3}}{\left[q\left|K_{a b}\right| q\right\rangle^{3}} \frac{\left[q\left|K_{a b}\right| c\right\rangle}{\left[q\left|K_{a b}\right| f\right\rangle}\right),
\end{array}
$$

where this corresponds to the double pole factorisation in fig. 3.3. The second helicity configuration of fig. 3.3 can be calculated via the symmetry principles derived from the six-point all-plus eq. (3.30). The sum of both helicity configurations gives the full $s_{a b}$ contribution to the rational piece.

For $C_{s b}^{\alpha^{-} \beta^{+}}$, however, there are useful cancellations between the two helicity choices for $\alpha$ and $\beta$ before integration. Defining $C_{s b}=C_{s b}^{\alpha^{-} \beta^{+}}+C_{s b}^{\alpha^{+} \beta^{-}}$, the full contribution from the square bracket factorisation is

$$
\left.\left.C_{s b}\right|_{\mathbb{Q}}=\frac{i}{9} \frac{[q a][q b]}{\langle a b\rangle} \frac{\left[f\left|K_{a b}\right| q\right\rangle^{2}}{\left[q\left|K_{a b}\right| q\right\rangle^{2} t_{c d e}}\left(\frac{\left\langle q\left|K_{a b}\right| c\right][c d]}{\langle d e\rangle\left\langle q\left|K_{a b} K_{c d}\right| e\right\rangle}-\frac{[d e][e f]}{\langle c d\rangle[f|k| c\rangle}+\frac{[c e]}{\langle c d\rangle\langle d e\rangle}\right)\right]
$$

\footnotetext{
${ }^{1}$ The restriction $\left.\right|_{Q}$ means only the finite rational functions of the spinor variables are kept.
} 


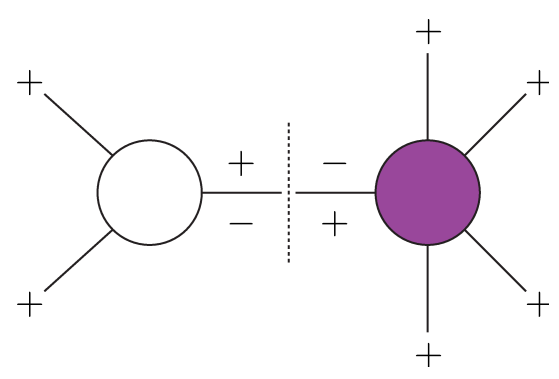

Figure 3.3: The one-loop to one-loop factorisation that corresponds to the factorisations derived in the current, $C_{d p}$ and $C_{s b}$. $C_{d p}$ corresponds to the factorisation involving the one-loop three-point all-plus and the one-loop five-point single-minus.

The expression for $C_{n f}^{\alpha^{-} \beta^{+}}$is quite complicated and is given in (C.46). Having integrated the diagram corresponding to the $s_{a b}$ pole, by calculating the residues of the excited diagrams via recursion, and subsequently summing over all contributing diagrams we recover $R_{6: 1}^{(2)}$. This form of the rational piece has the correct symmetry and $\lambda_{q}=\lambda_{\eta}$ independence, however this is not explicit, instead we reconstruct a form of the function that is manifestly independent of $\lambda_{q}=\lambda_{\eta}$, and is free of spurious poles.

\section{7 $\lambda_{q}$ Independent $R_{6: 1}^{(2)}$}

In this section we outline the method used to generate a manifestly $\lambda_{q}$-independent rational function. To distinguish between the two we write $R_{6: 1}^{(2)}$ for the manifestly $\lambda_{q}$-independent function and $R_{6: 1}^{(2)}(q)$ for the rational function where this fact is not made explicit.

We can obtain a form for $R_{6: 1}^{(2)}$ which is explicitly independent of $q$, has manifest cyclic symmetry and no spurious poles by reconstructing it from its factorisations. At this stage, we wish to capture the double pole structures and multiparticle pole structures from the factorisations and fix the remaining single pole contributions with an ansatz and reference to $R_{6: 1}^{(2)}(q)$. This can be achieved by considering the one-loop to one-loop factorisation channels ${ }^{1}$ for one configuration of external legs and perform a sum over a cycling of the legs. The relevant multiparticle and

\footnotetext{
${ }^{1}$ which source the multiparticle and double poles
} 
double pole factorisations are,

$$
\begin{aligned}
& G_{6: 1}^{t} \rightarrow \sum_{Z_{6}} A_{4: 1}^{(1)}\left(a^{+}, b^{+}, c^{+}, k^{ \pm}\right) \frac{-1}{t_{a b c}} A_{4: 1}^{(1)}\left(-k^{\mp}, d^{+}, e^{+}, f^{+}\right), \\
& G_{6: 1}^{d} \rightarrow \sum_{Z_{6}} A_{3: 1}^{(1)}\left(a^{+}, b^{+}, k^{+}\right) \frac{-1}{s_{a b}} A_{5: 1}^{(1)}\left(-k^{-}, c^{+}, d^{+}, e^{+}, f^{+}\right) .
\end{aligned}
$$

Note that the other helicity configuration of (3.40) is not considered since it is a single pole contribution and will be recovered when we fix the single pole terms with an ansatz.

These expressions are $q$-independent, but involve spurious poles that obscure the true singularity structure of the amplitude. In eliminating the spurious poles, we systematically obtain all the possible singularity structures 1 that one expects from a rational function with double poles. Performing the spurious pole cancellation stratifies the pole structure and the spurious poles can only be cancelled by exposing and then grouping together the physical poles. Following a great deal of cancellation, we obtain the pole structures $G_{6: 1}^{1}$ through to $G_{6: 1}^{4}$, for the six-point rational term包.

$$
R_{6: 1}^{\mathrm{fact}}=\frac{i}{9} \frac{1}{\langle 12\rangle\langle 23\rangle\langle 34\rangle\langle 45\rangle\langle 56\rangle\langle 61\rangle}\left(G_{6: 1}^{1}+G_{6: 1}^{2}+G_{6: 1}^{3}+G_{6: 1}^{4}\right)
$$

where

$$
\begin{aligned}
G_{6: 1}^{1} & =\frac{s_{c d} s_{d f}\left\langle f\left|a K_{a b c}\right| e\right\rangle}{\langle f e\rangle t_{a b c}}+\frac{s_{a c} s_{d c}\left\langle a\left|f K_{d e f}\right| b\right\rangle}{\langle a b\rangle t_{d e f}}, \\
G_{6: 1}^{2} & =\frac{[a b][f e]}{\langle a b\rangle\langle f e\rangle}\langle a e\rangle^{2}\langle f b\rangle^{2}+\frac{1}{2} \frac{[a f][c d]}{\langle a f\rangle\langle c d\rangle}\langle a c\rangle^{2}\langle d f\rangle^{2}, \\
G_{6: 1}^{3} & =\frac{s_{d f}\langle a f\rangle\langle c d\rangle[c a][d f]}{t_{a b c}}
\end{aligned}
$$

\footnotetext{
${ }^{1}$ with the exception of single poles.

${ }^{2}$ and $G_{7: 1}^{1}$ through to $G_{7: 1}^{6}$ for $R_{7: 1}^{(2)}$ shown in section 3.8
} 
and

$$
G_{6: 1}^{4}=\frac{\langle a|b e| f\rangle t_{d e f}}{\langle a f\rangle} .
$$

The remaining singular structure of the rational piece are the single poles. By extracting the double pole and multiparticle pole factorisations from the full rational function $R_{6: 1}^{(2)}(q)$, we isolate the simple pole contributions by considering the difference between $R_{6}^{(2)}(q)$ and the factorisation channels, $R_{6: 1}^{(2)}(q)-\left(G_{6: 1}^{1}+\right.$ $\left.\ldots+G_{6: 1}^{4}\right)$. We can fit this function via the following method. The simple pole contributions will arise in the Parke-Taylor denominator and so we can posit an ansatz for the numerator then solve for the coefficients for each term. The amplitude has momentum weight $\left[A_{6}^{(2)}\right]=-2$, by factoring out the Parke-Taylor denominator we require the numerator to have momentum weight four. The numerator ansatz must be a linear combination of all possible Lorentz invariant products that have momentum weight four. The numerical coefficients of the terms in the ansatz can then be solved for via a matrix inversion problem. We find that the simple pole numerator terms are $G_{6}^{5}$. There is a great deal of freedom in solving for the coefficients. Although there may be an optimal way of solving for these coefficients, we choose a solution that gives manageable coefficients.

$$
\begin{aligned}
G_{6: 1}^{5}= & 2 s_{a c}^{2}+s_{e b}^{2}+s_{a b}\left(-3 s_{a c}-2 s_{a d}+6 s_{a e}+4 s_{b c}+s_{b d}+2 s_{b e}+4 s_{b f}+7 s_{c d}-s_{c e}-s_{d e}+3 s_{d f}\right) \\
& +s_{a c}\left(2 s_{a d}+3 s_{a e}-2 s_{b d}-s_{b e}+s_{c f}-\frac{5}{2} s_{d f}\right)+\frac{3}{2} s_{a d} s_{b e} \\
& -8\langle b c\rangle[c d]\langle d e\rangle[e b]+5\langle f a\rangle[a c]\langle c d\rangle[d f]
\end{aligned}
$$

leading to the final expression,

$$
R_{6: 1}^{(2)}=\frac{i}{9} \sum_{Z_{6}} \frac{G_{6: 1}^{1}+G_{6: 1}^{2}+G_{6: 1}^{3}+G_{6: 1}^{4}+G_{6: 1}^{5}}{\langle 12\rangle\langle 23\rangle\langle 34\rangle\langle 45\rangle\langle 56\rangle\langle 61\rangle} .
$$

This was confirmed in an independent calculation [98] and has a simpler form than $[87]$. 


\subsection{The Seven-Point Rational Piece}

The seven-point rational piece is calculated in an identical fashion. The sevenpoint current $\tau_{7: 1}^{(1)}\left(\alpha^{-}, \beta^{+}, c^{+}, d^{+}, e^{+}, f^{+}, g^{+}\right)$, is built from the corresponding sevenpoint single-minus amplitude [38] just as the six-point current was built from the six-point amplitude. Moreover, the complex shift excites tree to two-loop factorisations for which $R_{6: 1}^{(2)}$ is required. Shifting the same legs, $p_{a}, p_{b}$ and $p_{c}$, we excite the following factorisations. The simple pole contributions to $R_{7: 1}^{(2)}$ arise in the multiparticle one-loop to one-loop factorisations that we calculate using standard recursive techniques,

$$
\begin{aligned}
& \sum_{h= \pm} A_{5: 1}^{(1)}\left(e, f, g, \hat{a}, \hat{K}^{h}\right) \frac{-1}{K^{2}} A_{4: 1}^{(1)}\left(-\hat{K}^{-h}, \hat{b}, \hat{c}, d\right) \\
& \sum_{h= \pm} A_{5: 1}^{(1)}\left(f, g, \hat{a}, \hat{b}, \hat{K}^{h}\right) \frac{-1}{K^{2}} A_{4: 1}^{(1)}\left(-\hat{K}^{-h}, \hat{c}, d, e\right)
\end{aligned}
$$

and all the tree to two-loop factorisations,

$$
\begin{array}{r}
A_{3}^{(0)}\left(g, \hat{a}, \hat{K}^{-}\right) \frac{-1}{K^{2}} R_{6: 1}^{(2)}\left(-\hat{K}^{+}, \hat{b}, \hat{c}, d, e, f\right), \\
A_{3}^{(0)}\left(\hat{a}, \hat{b}, \hat{K}^{-}\right) \frac{-1}{K^{2}} R_{6: 1}^{(2)}\left(-\hat{K}^{+}, \hat{c}, d, e, f, g\right), \\
R_{6: 1}^{(2)}\left(-\hat{K}^{+}, d, e, f, g, \hat{a}\right) \frac{-1}{K^{2}} A_{3}^{(0)}\left(\hat{b}, \hat{c}, \hat{K}^{-}\right), \\
R_{6: 1}^{(2)}\left(-\hat{K}^{+}, e, f, g, \hat{a}, \hat{b}\right) \frac{-1}{K^{2}} A_{3}^{(0)}\left(\hat{c}, d, \hat{K}^{-}\right),
\end{array}
$$

where $R_{6: 1}^{(2)}$ refers to the rational part of the leading in colour six gluon amplitude calculated in the previous section.

The remaining factorisations are the collinear one-loop to one-loop factorisations that involve the three-point one-loop all-plus vertex and are captured by 
performing augmented recursion:

$$
\begin{aligned}
& A_{3: 1}^{(1)}\left(g, \hat{a}, \hat{K}^{\lambda}\right) \frac{-1}{K^{2}} A_{6: 1}^{(1)}\left(-\hat{K}^{-\lambda}, \hat{b}, \hat{c}, d, e, f\right) \\
& A_{3: 1}^{(1)}\left(\hat{a}, \hat{b}, \hat{K}^{\lambda}\right) \frac{-1}{K^{2}} A_{6: 1}^{(1)}\left(-\hat{K}^{-\lambda}, \hat{c}, d, e, f, g\right) \\
& A_{3: 1}^{(1)}\left(\hat{b}, \hat{c}, \hat{K}^{\lambda}\right) \frac{-1}{K^{2}} A_{6: 1}^{(1)}\left(-\hat{K}^{-\lambda}, d, e, f, g, \hat{a}\right) \\
& A_{3: 1}^{(1)}\left(\hat{c}, d, \hat{K}^{\lambda}\right) \frac{-1}{K^{2}} A_{6: 1}^{(1)}\left(-\hat{K}^{-\lambda}, e, f, g, \hat{a}, \hat{b}\right)
\end{aligned}
$$

Taking $\alpha$ and $\beta$ off-shell in an identical way to the previous methods, leads to the double pole piece and the square pole pieces that recover the leading factorisations,

$$
\begin{aligned}
\tau_{7: 1 d p}^{(1), \alpha^{-} \beta^{+}} & \left(\alpha^{-}, \beta^{+}, c^{+}, d^{+}, e^{+}, f^{+}, g^{+}\right)=\frac{i}{18} \frac{\langle\alpha q\rangle^{2}}{\langle\beta q\rangle^{2}} \frac{\langle q|\alpha \beta| q\rangle}{\left[q\left|k_{\alpha \beta}\right| q\right\rangle^{2}} \frac{1}{s_{\alpha \beta}}\left(\frac{\langle g e\rangle[g f]\left[q\left|k_{\alpha \beta}\right| f\right\rangle^{3}}{\langle c d\rangle\langle d e\rangle\langle e f\rangle^{2}\langle f d\rangle^{2}\left[q\left|k_{\alpha \beta}\right| c\right\rangle}\right. \\
& +\frac{\langle c e\rangle[c d]\left[q\left|k_{\alpha \beta}\right| d\right\rangle^{3}}{\langle e f\rangle\langle f g\rangle\langle c d\rangle^{2}\langle d e\rangle^{2}\left[q\left|k_{\alpha \beta}\right| g\right\rangle}-\frac{\langle f d\rangle\left[q\left|k_{\alpha \beta}\right| e\right\rangle^{3}\left[q\left|k_{\alpha \beta} K_{c d}\right| e\right]}{\langle c d\rangle\langle f g\rangle\langle d e\rangle^{2}\langle e f\rangle^{2}\left[q\left|k_{\alpha \beta}\right| c\right\rangle\left[q\left|k_{\alpha \beta}\right| g\right\rangle} \\
& +\frac{\left[q\left|k_{\alpha \beta}\right| c\right\rangle^{2}\left[q\left|k_{\alpha \beta} K_{d e}\right| c\right]^{3}}{\langle f g\rangle\langle d e\rangle^{2}\left[q\left|k_{\alpha \beta}\right| g\right\rangle\left[q\left|k_{\alpha \beta} K_{f g} K_{d e}\right| c\right\rangle\left[q\left|k_{\alpha \beta} K_{\alpha \beta c} K_{d e}\right| f\right\rangle} \\
& +\frac{[c g]^{3}\left[q\left|k_{\alpha \beta}\right| c\right\rangle^{2}\left[f\left|K_{d e} K_{e f}\right| g\right]}{\langle d e\rangle\langle e f\rangle\left[c\left|k_{\alpha \beta}\right| g\right\rangle\left[d\left|K_{e f}\right| g\right\rangle\left[c\left|k_{\alpha \beta}\right| c\right\rangle t_{d e f}}+\frac{s_{d e}[c d][c g]^{3}\left[q\left|k_{\alpha \beta}\right| c\right\rangle^{3}}{\langle d e\rangle\langle e f\rangle\left[c\left|k_{\alpha \beta}\right| g\right\rangle\left[c\left|k_{\alpha \beta}\right| c\right\rangle\left[q\left|k_{\alpha \beta} K_{\alpha \beta c} K_{d e}\right| f\right\rangle t_{d e f}} \\
& \left.-\frac{\left[q\left|k_{\alpha \beta} K_{c d}\right| g\right]^{3}}{\left.\langle c d\rangle\langle e f\rangle^{2}\left[g\left|K_{e f}\right| d\right\rangle\left[q\left|k_{\alpha \beta}\right| c\right\rangle t_{e f g}\right]}\right)
\end{aligned}
$$

and,

$$
\begin{gathered}
\tau_{7: 1 s b}^{(1), \alpha^{-} \beta^{+}}\left(\alpha^{-}, \beta^{+}, c^{+}, d^{+}, e^{+}, f^{+}, g^{+}\right)=\frac{i}{18} \frac{\langle\alpha q\rangle^{2}}{\langle\beta q\rangle^{2}} \frac{[q|\beta \alpha| q][q|\beta| q\rangle^{2}}{\left[q\left|k_{\alpha \beta}\right| q\right\rangle[q|\alpha| q\rangle^{2}} \frac{1}{s_{\alpha \beta}} \frac{\left[g\left|k_{\alpha \beta}\right| q\right\rangle}{\left[q\left|k_{\alpha \beta}\right| g\right\rangle\langle c d\rangle\langle d e\rangle\langle e f\rangle} \\
\times\left([c f]-\frac{s_{d e}\left[g\left|K_{e f} K_{c d} k_{\alpha \beta}\right| q\right\rangle}{\left[g\left|k_{\alpha \beta}\right| c\right\rangle\left\langle q\left|k_{\alpha \beta} K_{\alpha \beta g}\right| f\right\rangle}+\frac{\left[c\left|K_{d e} K_{c d} k_{\alpha \beta}\right| q\right\rangle}{\left\langle q\left|k_{\alpha \beta} K_{\alpha \beta g}\right| f\right\rangle}+\frac{\left[f\left|K_{d e} K_{e f}\right| g\right]}{\left[g\left|k_{\alpha \beta}\right| c\right\rangle}\right) .
\end{gathered}
$$

The remaining non-factorising terms, $\tau_{7: 1 n f}^{(1), \alpha^{-} \beta^{+}}$, are the combination of the terms in the seven-gluon single minus amplitude that are finite in $s_{\alpha \beta}$, and the off-shell corrections that lead to $\tau_{7: 1 d p}^{(1), \alpha^{-} \beta^{+}}$and $\tau_{7: 1 s b}^{(1), \alpha^{-} \beta^{+}}$. 


\subsection{The Seven-Point Rational Piece}

Integrating the current into the triangle diagram, fig. 2.7, and calculating the residues of the four excited diagrams in the $s_{a b}$ channel gives the rational term encompassing the information from the double pole and its subleading pole. Summing the double pole rational term and the single pole factorisation channels that did not require augmented recursion, gives the following function after reconstruction,

$$
\begin{aligned}
& G_{7: 1}^{1}= \frac{\langle g a\rangle}{t_{a b c} t_{e f g}}\left(\frac{\langle c d\rangle[e g]\left[d\left|K_{a b c}\right| e\right\rangle\left[a\left|K_{a b c}\right| e\right\rangle\left[c\left|K_{a b c}\right| f\right\rangle}{\langle e f\rangle}-\frac{\langle d e\rangle[c a]\left[d\left|K_{e f g}\right| c\right\rangle\left[g\left|K_{e f g}\right| c\right\rangle\left[e\left|K_{e f g}\right| b\right\rangle}{\langle b c\rangle}\right. \\
&\left.+\frac{\langle e f\rangle\langle c d\rangle[c a][f g]\left[e\left|K_{e f g}\right| a\right\rangle\left[d\left|K_{e f g}\right| b\right\rangle}{\langle a b\rangle}-\frac{\langle b c\rangle\langle d e\rangle[e g][a b]\left[c\left|K_{a b c}\right| g\right\rangle\left[d\left|K_{a b c}\right| f\right\rangle}{\langle f g\rangle}\right), \\
& G_{7: 1}^{2}= \frac{1}{t_{a b c} t_{e f g}} s_{c d} s_{d e}\langle g a\rangle\left[g\left|K_{e f g} K_{a b c}\right| a\right], \\
& G_{7: 1}^{3}= \frac{1}{t_{c d e}}\left(s_{c e}\left(\frac{s_{e f}\left\langle c\left|K_{a b} K_{f g a}\right| d\right\rangle}{\langle c d\rangle}-\frac{s_{b c}\left\langle e\left|K_{f g} K_{g a b}\right| d\right\rangle}{\langle d e\rangle}\right)+\frac{\langle e f\rangle\langle b c\rangle[f b]\left[c\left|K_{c d e}\right| g\right\rangle\left[e\left|K_{c d e}\right| a\right\rangle}{\langle g a\rangle}\right. \\
&\left.\quad+\frac{\langle b c\rangle\left[c\left|K_{c d e}\right| b\right\rangle\left[e\left|K_{c d e}\right| a\right\rangle\left[b\left|K_{f g}\right| e\right\rangle}{\langle a b\rangle}+\frac{\langle e f\rangle\left[e\left|K_{c d e}\right| f\right\rangle\left[c\left|K_{c d e}\right| g\right\rangle\left[f\left|K_{a b}\right| c\right\rangle}{\langle f g\rangle}\right) \\
& G_{7: 1}^{4}=\frac{[g a]}{\langle g a\rangle}\langle g e\rangle\langle a e\rangle\left(\frac{[d e]}{\langle d e\rangle}\langle d g\rangle\langle d a\rangle+\frac{[e f]}{\langle e f\rangle}\langle f g\rangle\langle f a\rangle\right) \\
& G_{7: 1}^{5}=\frac{1}{t_{c d e}}\left([c e]\left(\langle e f\rangle[d f]\left\langle c\left|K_{a b} K_{f g a}\right| d\right\rangle+\langle b c\rangle[d b]\left\langle e\left|K_{f g} K_{g a b}\right| d\right\rangle\right)\right. \\
& G_{7: 1}^{6}=\frac{1}{\langle g a\rangle}\left(\left\langle g\left|f K_{b c}\right| a\right\rangle t_{e f g}-\left\langle a\left|b K_{e f}\right| g\right\rangle t_{a b c}\right),
\end{aligned}
$$

and

$$
\begin{aligned}
G_{7: 1}^{7}= & s_{b f}^{2}-2 s_{g a}^{2}-3 s_{d b} s_{d f}+4 s_{d a} s_{d g}-6 s_{a c} s_{e g}+7\left(s_{e b} s_{f c}+s_{e a} s_{g c}\right)+s_{a b} s_{f g}+3 s_{f a} s_{g b} \\
& +s_{c e}\left(s_{c f}+s_{e b}-4\left(s_{a b}+s_{f g}+s_{g a}\right)+5\left[d\left|K_{g a}\right| d\right\rangle\right) \\
& +4[e|b c f| e\rangle-2[f|g a b| f\rangle+3[g|b a f| g\rangle+2[g|c e a| g\rangle
\end{aligned}
$$




\section{ANALYTIC ALL-PLUS-HELICITY GLUON AMPLITUDES IN QCD}

the full function in this case is

$$
R_{7: 1}^{(2)}=-\frac{i}{9} \sum_{\text {cyclic perms }} \frac{G_{7: 1}^{1}+G_{7: 1}^{2}+G_{7: 1}^{3}+G_{7: 1}^{4}+G_{7: 1}^{5}+G_{7: 1}^{6}+G_{7: 1}^{7}}{\langle 12\rangle\langle 23\rangle\langle 34\rangle\langle 45\rangle\langle 56\rangle\langle 67\rangle\langle 71\rangle} .
$$

This expression has the full cyclic and flip symmetries required and has all the correct factorisations by construction. It additionally satisfies the collinear limits that reduce $R_{7: 1}^{(2)}$ to $R_{6: 1}^{(2)}$. It been generated under the assumption that the shifted rational function vanishes at infinity: if this had been unjustified we would not have generated a function with the appropriate symmetries. Notice that the singularity structures found in the seven-point are the most This completes the seven-point calculation.

\subsection{Conclusion}

In this section we have detailed the construction of the leading in colour twoloop six-point all-plus gluon scattering amplitude and presented compact analytic expressions for the six- and seven-point cases. Our methods are based upon the understanding and utilisation of the singular structure an on-shell amplitude possesses, specialised to the all-plus case.

In section 3.2 we reviewed the leading in colour all-plus amplitude and detailed how the all-plus amplitude can be factorised into pieces based on its singular structure. The amplitude can be separated into IR singular terms and finite terms that are a sum of functions that contain branch cuts and poles.

In section 3.3 we reviewed a derivation of the IR singular terms and the finite polylogarithmic terms from four-dimensional unitarity cuts. We saw that using four-dimensional cuts drastically reduces the complexity of the diagrams that have to be considered to capture the full branch cut singularity structure. The only non-vanishing four-dimensional cuts of the amplitude are the configurations for which the one-loop all-plus amplitude enters the cut integrals. This is a purely rational function to $\mathcal{O}(\epsilon)$ and can be treated as a vertex, thus the effective integral basis of the two-loop all-plus amplitude is that of a one-loop amplitude. Utilising generalised unitarity in four-dimensions to compute the cuts of the box, triangle and bubble integrals confirmed the theorems for the IR singularity structure of 
two-loop amplitudes [33] and gave an all- $n$ expression for the finite polylogarithmic terms [87].

Section 3.4 determined the rational term of the two-loop six-gluon all-plus amplitude. Using complex recursion and augmented recursion, we determined the excited factorisations that contribute to the rational piece, importantly determining the subleading pole structure belonging to the double pole, which is unavailable to general factorisation theorems. As of yet, augmented recursion does not determine the subleading pole structure in general and so it must be applied on a case by case basis. Despite this we have shown its success again at seven-point by deriving the rational part of the seven-point all-plus and presenting the result in section 3.8 .

This is the first seven-point amplitude to be computed at the two-loop level and shows the efficiency of employing four-dimensional unitarity. Despite its success, the methods in this context are only geared to determining leading in colour amplitudes. Given the relative simplicity of the calculations required to compute the five-point all-plus amplitude at leading in colour, we were motivated to understand how four-dimensional unitarity could be utilised at subleading in colour. In the next chapter, we extend four-dimensional unitarity and recursion to the realms of subleading in colour partial amplitudes by making use of the group theory $U(1)$-decoupling relations and newly uncovered compact forms of the subleading in colour one-loop all-plus amplitudes. 
3. ANALYTIC ALL-PLUS-HELICITY GLUON AMPLITUDES IN QCD 


\section{Chapter 4}

\section{Colour Dressed Unitarity and Recursion for Yang-Mills Two-Loop All-Plus Amplitudes}

\subsection{Introduction}

Two-loop amplitudes represent the frontier of phenomenological calculations in Yang-Mills. The added degree of complexity introduced by an additional loop integration, including the appearance of more complicated singularity structures, poses a significant barrier to obtaining compact analytic formulae. This is compounded by the difficulties that arise with considering the non-planar (sub)subleading in colour amplitudes, that were easily related to the leading in colour amplitude at one-loop. In order to calculate partonic cross sections, a sum over colour must be performed including the (sub-)subleading information. The implication being, full colour information is of vital importance in extracting useful data at particle colliders involving hadrons. Apart from the obvious application to experiment, nonetheless it is interesting to investigate the kinematic structure of subleading partial amplitudes especially at the two-loop level. One-loop partial amplitudes have the property that all subleading in colour partial amplitudes are related to the leading in colour partial amplitude via $U(1)$-decoupling identities. The same is not true at the two-loop level. As discussed in subsection 2.3.2, decoupling identities [128] cannot determine the subleading structure alone. Even 


\section{COLOUR DRESSED UNITARITY AND RECURSION FOR YANG-MILLS TWO-LOOP ALL-PLUS AMPLITUDES}

with the inclusion of Edison and Nauclich's group theory relations [15, 16], the full amplitude is not available once the leading in colour amplitude has been determined. Therefore, it is worthwhile to explore methods of determining subleading in colour partial amplitudes beyond one-loop.

The story of full colour two-loop amplitudes is entwined with the narrative on the leading in colour amplitudes for obvious reasons, although in almost all cases the leading in colour result preceded the full colour result. This is due in part to the presence of more complicated non-planar integrals that are required to determine the subleading in colour partial amplitudes and indeed there is a larger class of master integrals.

The two-loop four-point all-plus amplitude [70] was the only two-loop pure Yang-Mills helicity amplitude to have been computed to full colour at the first time of asking. Utilising $D$-dimensional unitarity, the authors considered cuts of the two-loop amplitude where the cut integral contained full colour amplitudes using a colour decomposition akin to DDM [14]. Following on from these methods, all the helicity gluon amplitudes at two-loop four-point were calculated [92] finding complete agreement with [93].

At five-point, the first full colour result came in the form of the all-plus 95 . The authors circumvented issues with non-planar integrals by relating them to planar five-point two-loop integrals computed in the leading case [94. Subsequently, the computation of the same full colour amplitude was carried out analytically in ref. [110]. In this case, an $S U\left(N_{c}\right)$ colour trace basis was adopted in combination with integration-by-parts (IBP) identities and differential equations to solve for a basis of six master integrals, two planar and the rest non-planar. Only one of the integrals, a non-planar double-pentagon required a new calculation [103, 159]. In combination with finite field reconstruction methods, a compact analytic form was found for the the subleading partial amplitude $A_{5: 3}^{(2)}$, in terms of integral functions multiplied by rational functions of the momenta, plus a finite hard function. There is another partial amplitude at sub-subleading order present in both $U\left(N_{c}\right)$ and $S U\left(N_{c}\right)$ theories, a term we have called $A_{5: 1 B}^{(2)}$, that was not presented in an explicit form. Despite the fact there was no explicit form detailed for this amplitude it can be related to the leading and subleading partial amplitudes presented in ref. [110] via a group theory relation [15, 16]. 
Each result found at subleading in colour was derived using $D$-dimensional unitarity. However, the bottlenecks in calculations via approach lies in finding a basis of integrals to describe the partial amplitudes and the evaluation of higherpoint integrals, including those of a non-planar nature. At the level of the all-plus amplitude, we circumvent these issues with four-dimensional unitarity. Since all external gluons have positive helicity, this strongly constrains the helicity configurations of the diagrams that one can write down. In addition, every cut integral describing the discontinuity of the two-loop diagrams must contain the one-loop all-plus amplitude. As the one-loop all-plus amplitude is purely a rational function, we may treat it as a vertex and use a one-loop integral basis to describe the discontinuity of the two-loop amplitude.

The following chapter is based on work performed in [89] in which we extend our methods to a process of colour dressed unitarity and recursion. We develop a method of directly calculating the partial amplitudes at all orders in $N_{c}$ to fully reconstruct the full colour two-loop five-point all-plus amplitude.

The calculation is founded on the principles of four-dimensional unitarity used to determine a variety of amplitudes at leading order in the number of colours. In the leading case, the external legs are fixed in a cyclic order when considering the cuts and residues of the amplitude. This is implicitly respecting the ordering of the gluon generators present in the trace structure multiplying the leading in colour partial amplitude. In this calculation, we simply replace the partial amplitudes present in the cuts and factorisations with their full colour counterparts and perform the colour algebra accordingly. This replacement ensures that by considering all cuts and all residues 1 of the amplitude we reconstruct the full colour amplitude. We choose to determine the full colour amplitude in $U\left(N_{c}\right)$ Yang-Mills using a colour trace basis where the colour decomposition is in terms of traces of $U\left(N_{c}\right)$ generators, $T^{a}$, rather than contractions of the structure constants $f^{a b c}$.

This amplitude is fully crossing symmetric which makes computation relatively more tractable but nonetheless is a valuable laboratory for studying the properties of gluon scattering. The all-plus amplitude has a singular structure which is known from general theorems [32, 33] together with a finite remainder part. We present a form for the finite part of the partial amplitudes which is

\footnotetext{
${ }^{1}$ relating to the rational part of the amplitude
} 


\section{COLOUR DRESSED UNITARITY AND RECURSION FOR YANG-MILLS TWO-LOOP ALL-PLUS AMPLITUDES}

a simple combination of polylogarithms together with rational terms. Our results are in complete agreement with the results recently computed by Badger et. al. [110] and are consistent with constraints imposed by group theoretical arguments [15, 16].

The chapter is organised in the following way. In section 4.2, we review the colour decompositions of tree-level and one-loop amplitudes in $U\left(N_{c}\right)$ Yang-Mills and describe the $U(1)$-decoupling identities present amongst those amplitudes. We also present compact formulae for the subleading partial amplitudes of the all-plus and single-minus one-loop amplitudes and demonstrate a way of characterising the singular nature of the subleading in colour amplitudes at one-, and two-loops. In section 4.3, we present the colour decomposition for twoloop amplitudes in $U\left(N_{c}\right)$ and $S U\left(N_{c}\right)$ Yang-Mills and show relations between the various partial amplitudes from decoupling identities. We also show that the sub-subleading amplitude $A_{5: 1 B}^{(2)}$ cannot be related to the other partial amplitudes via decoupling identities but requires the use of another group theory result [15] [16]. In section 4.4, we briefly show how we organise the kinematics of the amplitude via its singularity structure. Thereafter, section 4.5 deals with the cut-constructible parts of the amplitude, illustrating the all- $n$ prescription for the all-plus amplitude, then specialising to the full colour five-point results. We reproduce the IR singular structure predicted by Catani and present compact formulae for the leading and (sub-)subleading polylogarithmic terms at $n=5$. Finally, section 4.6 shows how to extend augmented recursion to a full colour formalism. In particular, it highlights the two independent doubly off-shell currents that are required to compute the full pole structure of the amplitude at all orders in colour at five-point. This direct calculation culminates in compact formulae for the rational parts of the $S U\left(N_{c}\right)$ amplitude. 


\subsection{Colour Decomposition of Amplitudes for Colour Dressed Unitarity and Recursion}

The natural extension of four-dimensional unitarity and recursion from its application to leading in colour partial amplitudes to fully colour dressed amplitudes is found, in essence, with the replacement of partial amplitudes with colour dressed amplitudes. Using the same philosophy applied at leading in colour, this extension is applied in the context of obtaining the full colour two-loop five-point all-plus amplitude. In the previous section for example, to obtain the leading in colour result, a basis of integral functions was selected and the corresponding diagrams systematically cut. The diagrams are constrained by the symmetry of the trace structure they multiply, for example a partial amplitude may be accompanied by the following trace structure,

$$
\operatorname{Tr}\left(T^{a_{1}} T^{a_{1}} \cdots T^{a_{n}}\right) A_{n: 1}\left(p_{1}, p_{2}, \cdots, p_{n}\right) .
$$

The trace is invariant under cycles of the the generators of the external legs which fixes the legs of the amplitude to have the corresponding ordering and cyclic symmetry. Thus in that case we only consider the basis of diagrams that respect this ordering. When considering the extension of this to a full colour result, the natural question to ask is what diagrams one should consider when determining the cut-constructible pieces at (sub-)subleading order? The usual methodology when dealing with gluon scattering amplitudes is to separate colour and kinematics and treat them as separate entities. In the method of colour dressed unitarity we utilise unitarity and recursion together with colour in an effort to understand which types of partial amplitudes contribute to a specific order in the full colour decomposition. In this way we can determine all the partial amplitudes at play in the full colour amplitude.

We choose to describe the full colour amplitudes using the gauge group, $U\left(N_{c}\right)=S U\left(N_{c}\right) \times U(1)$. Despite introducing some unphysical $U\left(N_{c}\right)$ partial amplitudes, picking an external leg to be the $U(1)$-'photon' would mean the photon decouples from the gluons and the colour decomposition reduces to the 


\section{COLOUR DRESSED UNITARITY AND RECURSION FOR YANG-MILLS TWO-LOOP ALL-PLUS AMPLITUDES}

physical $S U\left(N_{c}\right)$ form. The freedom to include the $U(1)$-'photon' has the advantage of relating various leading and (sub-)subleading partial amplitudes to each other in order to sum to zero. This is a statement that the gluon and the 'photon' do not interact and so a sum of partial amplitudes that involve such a particle will vanish. These decoupling identities [128] act as important checks on the partial amplitudes that are calculated at each order in the number of colours. The $U\left(N_{c}\right)$ colour decompositions and decoupling identities are important to understand given they will appear in all subsequent calculations, and have been reviewed in subsection 2.3.1 and subsection 2.3.2.

In order to compute the two-loop all-plus gluon amplitude, we require the full colour one-loop all-plus amplitude in both unitarity cuts and factorisation channels. Due to the decoupling identities, only the leading in colour one-loop partial amplitude [77] is required to fix the subleading one-loop partial amplitudes. However, expressing the one-loop subleading partial amplitudes via the decoupling identities gives rise to expressions that are built up of large sums. By considering Lorentz invariance, locality and the symmetries that the subleading in colour amplitudes must satisfy, it is possible to write down a more compact form of these rational partial amplitudes. We summarise the $n$-point expressions to $\mathcal{O}\left(\epsilon^{0}\right)$ below - presenting new compact formulae for the subleading partial amplitudes $A_{n: 2}^{(1)}$ and $A_{n: r}^{(1)}$,

$$
\begin{aligned}
& A_{n: 1}^{(1)}\left(1^{+}, 2^{+}, 3^{+}, \cdots, n^{+}\right)=-\frac{i}{3} \frac{1}{\langle 12\rangle\langle 23\rangle \cdots\langle n 1\rangle} \sum_{1 \leq i<j<k<l \leq n} \operatorname{tr}_{-}[i j k l]+\mathcal{O}(\epsilon), \\
& A_{n: 2}^{(1)}\left(1^{+} ; 2^{+}, 3^{+}, \cdots, n^{+}\right)=-i \frac{1}{\langle 23\rangle\langle 34\rangle \cdots\langle n 2\rangle} \sum_{2 \leq i<j \leq n}[1 i]\langle i j\rangle[j 1]+\mathcal{O}(\epsilon),
\end{aligned}
$$

and for $r \geq 3$,

$A_{n: r}^{(1)}\left(1^{+}, 2^{+}, \cdots, r-1^{+} ; r^{+} \cdots n^{+}\right)=-2 i \frac{\left(K_{1 \cdots r-1}^{2}\right)^{2}}{(\langle 12\rangle\langle 23\rangle \cdots\langle(r-1) 1\rangle)(\langle r(r+1)\rangle \cdots\langle n r\rangle)}+\mathcal{O}(\epsilon)$. 


\subsection{Colour Decomposition of Amplitudes for Colour Dressed Unitarity and Recursion}

These expressions are remarkably compact given the number of terms arising in the naive application of the decoupling identities (2.46). The number of terms in the numerator of a single $A_{n: 1}^{(1)}$ grows as,

$$
\frac{1}{24} n(n-1)(n-2)(n-3),
$$

while the summation over $C O P$ terms in (2.46) grows with $n$ as,

$$
\sim \frac{(n-1) !}{(r-2) !(n-r) !} \text {. }
$$

The naive application of the decoupling identities in determining the subleading partial amplitudes leads to huge sums of leading partial amplitudes. This is contrasted with the stark fact that (4.4) is made up of only one term.

The decoupling identities have their use in other ways, however. When computing the rational part of the full colour amplitude, we have seen that the twoloop all-plus amplitude contains double poles that must be treated with augmented recursion. To obtain the subleading pole contributions, we continued two gluons in the one-loop single-minus amplitude off-shell to produce a current that adequately captured the subleading pole behaviour. In the full colour extension to five-point, we consider the full colour single-minus five-point gluon amplitude in order to construct a full colour current. At subleading in colour we can understand a priori, through the decoupling identities, if the subleading in colour rational terms have double poles and require augmented recursion.

Beginning at one-loop, we know from the form of the leading in colour singleminus amplitude that double poles in the amplitudes can be seen explicitly. The five-point case [148],

$A_{5: 1}^{(1)}\left(1^{-}, 2^{+}, 3^{+}, 4^{+}, 5^{+}\right)=\frac{i}{3} \frac{1}{\langle 34\rangle^{2}}\left[-\frac{[25]^{3}}{[12][51]}+\frac{\langle 14\rangle^{3}[45]\langle 35\rangle}{\langle 12\rangle\langle 23\rangle\langle 45\rangle^{2}}-\frac{\langle 13\rangle^{3}[32]\langle 42\rangle}{\langle 15\rangle\langle 54\rangle\langle 32\rangle^{2}}\right]$,

has $\langle a b\rangle^{-2}$ singularities for $\langle a b\rangle=\langle 23\rangle,\langle 34\rangle$ and $\langle 45\rangle$.

We also know that factorisations as in fig. 4.1 are responsible for such double poles. 


\section{COLOUR DRESSED UNITARITY AND RECURSION FOR YANG-MILLS TWO-LOOP ALL-PLUS AMPLITUDES}

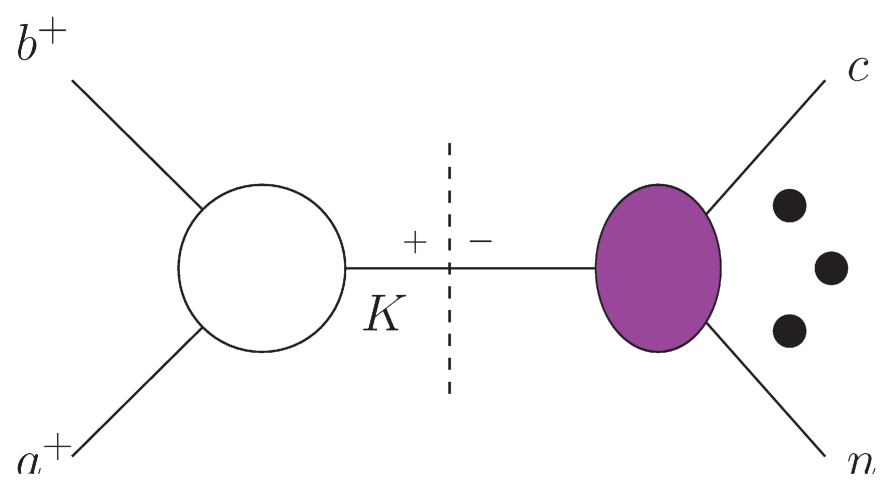

Figure 4.1: The origin of the double pole. The double pole arises due to the coincidence of the singularity arising in the one-loop three-point all-plus vertex, and the propagator defining the channel in the factorisation corresponding to $K^{2}=$ $s_{a b} \rightarrow 0$. The helicities on the right hand side of the factorisation are general.

The factorisation takes the form,

$$
\frac{V^{(1)}\left(a^{+}, b^{+}, K^{+}\right)}{s_{a b}} \times \frac{-1}{s_{a b}} \times A_{n-1: 1}^{(0)}\left(K^{-}, c^{-}, \cdots, n^{+}\right) \sim \frac{[a b]}{\langle a b\rangle^{2}} \times A_{n-1: 1}^{(0)}\left(K^{-}, c^{-}, \cdots, n^{+}\right),
$$

where,

$$
\frac{V^{(1)}\left(a^{+}, b^{+}, K^{+}\right)}{s_{a b}}=-\frac{i}{3} \frac{[a b][b K][K a]}{s_{a b}},
$$

is the one-loop three-point vertex [38].

Expressing the subleading partial amplitudes in terms of the leading case from the application of (2.46), naively indicates that the subleading in colour partial amplitude could also contain double poles. This method of obtaining the subleading amplitudes, despite ensuring they have the correct symmetries, introduces a great deal of redundancy in the expression obscuring the simplicity of the pole structure. However, as we have identified (4.8) as the factorisation sourcing of the double poles, we can use this to our advantage. By expressing the subleading in colour amplitude using the decoupling identities, relating it to the leading in colour amplitude, we may expose the double pole contributions from the leading

\footnotetext{
${ }^{1}$ As a small aside, the same analysis (4.8) is available to the all-plus amplitude with the a positive helicity on $c$. For $n>4$, the all-plus one-loop amplitude does not contain double poles since $A_{n-1: 1}^{(0)}\left(K^{-}, c^{+}, \cdots, n^{+}\right)$vanishes.
} 


\subsection{Colour Decomposition of Amplitudes for Colour Dressed Unitarity and Recursion}

partial amplitude using (4.8). This allows us to understand a priori if there are double poles at subleading orders. In fact, we show there are no double poles in the one-loop sub-leading partial amplitudes for $n>4$.

Consider the first subleading in colour partial amplitude, $A_{n: 2}^{(1)}\left(a_{1} ; b_{2}, b_{3}, b_{4}, \cdots b_{n}\right)$ and express this as a sum over the $A_{n: 1}^{(1)}$,

$$
A_{n: 2}^{(1)}\left(a_{1} ; b_{2}, b_{3}, \cdots b_{n}\right)=-A_{n: 1}^{(1)}\left(a_{1}, b_{2}, b_{3}, \cdots b_{n}\right)-A_{n: 1}^{(1)}\left(b_{2}, a_{1}, b_{3}, \cdots b_{n}\right) \cdots-A_{n: 1}^{(1)}\left(b_{2}, b_{3}, \cdots a_{1}, b_{n}\right),
$$

where the sum is over the $(n-1)$ distinct positions that $a_{1}$ may be inserted within $b_{2}, b_{3} \cdots b_{n}$. There are two cases for which the amplitude can pick up a complex double pole. The first is when the legs $a_{1}$ and $b_{2}$ are adjacent and have positive helicity, and the second is when the legs $b_{2}$ and $b_{3}$ are adjacent and have the same helicity.

If we consider the double pole in $\left\langle a_{1} b_{2}\right\rangle$, the double pole only arises in the first two terms of the decoupling identity (4.10) and will be of the form

$$
-\frac{V^{(1)}\left(a_{1}^{+}, b_{2}^{+}, K^{+}\right)}{s_{a_{1} b_{2}}^{2}} \times A_{n-1: 1}^{(0)}\left(K^{-}, b_{3}, \cdots b_{n}\right)-\frac{V^{(1)}\left(b_{2}^{+}, a_{1}^{+}, K^{+}\right)}{s_{a_{1} b_{2}}^{2}} \times A_{n-1: 1}^{(0)}\left(K^{-}, b_{3}, \cdots b_{n}\right),
$$

which vanishes since $V^{(1)}\left(a^{+}, b^{+}, K^{+}\right)$is antisymmetric. The double pole in $\left\langle b_{2} b_{3}\right\rangle$ also vanishes, but via a different route. Only the second term in (4.10) does not contribute and we obtain

$$
\begin{aligned}
-\frac{V^{(1)}\left(b_{2}^{+}, b_{3}^{+}, K^{+}\right)}{s_{b_{2} b_{3}}^{2}} \times\left(A_{n-1: 1}^{(0)}\left(a_{1}, K^{-}, b_{4}, \cdots b_{n}\right)+\right. & A_{n-1: 1}^{(0)}\left(K^{-}, a_{1}, b_{4}, \cdots b_{n}\right) \\
& \left.+\cdots+A_{n-1: 1}^{(0)}\left(K^{-}, b_{4}, \cdots a_{1}, b_{n}\right)\right) .
\end{aligned}
$$

This sum is exactly a tree-level decoupling identity (2.44) for $(n-1)$ legs that arises by setting the leg $a_{1}$ to be the $U(1)$-photon. Similar arguments show the vanishing of double poles for all $A_{n: r}^{(1)}$ with $r>1$.

This drastically simplifies the range of functions that we know could describe the subleading in colour partial amplitudes. The partial amplitudes are rational 


\section{COLOUR DRESSED UNITARITY AND RECURSION FOR YANG-MILLS TWO-LOOP ALL-PLUS AMPLITUDES}

functions with simple poles that must satisfy the symmetries of the trace structures they multiply and indeed they must carry the correct spinor weight for a given helicity configuration.

Using these facts we can find all- $n$ formulae for $A_{n: 2}^{(1)}\left(1^{-} ; 2^{+}, \cdots n^{+}\right)$and $A_{n: 3}^{(1)}\left(1^{-}, 2^{+} ; 3^{+}, \cdots n^{+}\right)$:

$$
A_{n: 2}^{(1)}\left(1^{-} ; 2^{+}, 3^{+}, \cdots, n^{+}\right)=\frac{-i \sum_{2 \leq i<j \leq n}\langle 1|i j| 1\rangle}{\langle 23\rangle\langle 34\rangle \cdots\langle(n-1) n\rangle\langle n 2\rangle},
$$

and

$$
A_{n: 3}^{(1)}\left(1^{-}, 2^{+} ; 3^{+}, \cdots, n^{+}\right)=\sum_{Z_{(3 \cdots n)}} \frac{i \sum_{2 \leq i<j \leq n}\langle 1|i j| 1\rangle}{\langle 23\rangle\langle 34\rangle \cdots\langle(n-1) n\rangle\langle n 2\rangle}
$$

where $Z_{(3 \cdots n)}$ is the set of cyclic permutations of the set $(3, \cdots n)$.

The vanishing of the $\left\langle b_{2} b_{3}\right\rangle$ double poles in (4.12) uses a tree-level identity, so we do not expect the argument to extend beyond one-loop. Specifically if we consider $A_{n: 2}^{(2)}\left(a_{1} ; b_{2}, b_{3}, b_{4}, \cdots b_{n}\right)$, a formula for the double pole in $\left\langle b_{2} b_{3}\right\rangle$ akin to (4.12) will exist but with the tree amplitudes $A_{n-1: 1}^{(0)}$ replaced by their one-loop equivalents $A_{n-1: 1}^{(1)}$. The combination of $A_{n-1: 1}^{(1)}$ is that of the decoupling identity (2.46) so the double pole does not vanish but instead is proportional to

$$
\frac{V^{(1)}\left(b_{2}^{+}, b_{3}^{+}, K^{+}\right)}{s_{b_{2} b_{3}}^{2}} \times A_{n-1: 2}^{(1)}\left(a_{1} ; K^{-}, b_{4}, \cdots b_{n}\right) \text {. }
$$

Specialising to the five-point case, we consider the non-vanishing (sub-)subleading partial amplitudes, $A_{5: 3}^{(2)}, A_{5: 1,1}^{(2)}$ and $A_{5: 1 B}^{(2)}$. The decoupling identities involving two generators becoming $U(1)$ generators gives a relation,

$$
A_{5: 1,1}^{(2)}\left(a_{1} ; b_{1} ; c_{1}, c_{2}, c_{3}\right)=-A_{5: 3}^{(2)}\left(a_{1}, b_{1} ; c_{1}, c_{2}, c_{3}\right)+\sum_{C O P\left\{a_{1}, b_{1}\right\}\left\{c_{1}, c_{2}, c_{3}\right\}} A_{5: 1}^{(2)}\left(a_{1}, b_{1}, c_{1}, c_{2}, c_{3}\right) .
$$




\subsection{Two-Loop Amplitudes}

Analysing the double pole structure, $\left\langle a_{1} b_{1}\right\rangle$, we have,

$$
\begin{aligned}
A_{5: 1,1}^{(2)}\left(a_{1} ; b_{1} ; c_{1}, c_{2}, c_{3}\right) & +\left.A_{5: 3}^{(2)}\left(a_{1}, b_{1} ; c_{1}, c_{2}, c_{3}\right)\right|_{\left\langle a_{1} b_{1}\right\rangle}=\frac{V_{3}^{(1)}\left(a_{1}^{+}, b_{1}^{+}, K^{+}\right)}{s_{a_{1} b_{1}}^{2}}\left[A_{5: 1}^{(1)}\left(a_{1}, b_{1}, c_{1}, c_{3}, c_{2}\right)\right. \\
& \left.+A_{5: 1}^{(1)}\left(a_{1}, b_{1}, c_{2}, c_{1}, c_{3}\right)+A_{5: 1}^{(1)}\left(a_{1}, b_{1}, c_{2}, c_{3}, c_{1}\right)+A_{5: 1}^{(1)}\left(a_{1}, b_{1}, c_{3}, c_{1}, c_{2}\right)\right]
\end{aligned}
$$

which implies that both $A_{5: 1,1}^{(2)}$ and $A_{5: 3}^{(2)}$ contain double poles. Furthermore, using Edison and Naculich's [15, 16] group theory relations shown in (4.24) to identify $A_{5: 1 B}^{(2)}$, this function will contain terms that have double poles in the rational piece. However, direct calculation reveals an interesting caveat to this statement, which is discussed at the end of section 4.6.

\subsection{Two-Loop Amplitudes}

In this section we briefly review the trace based colour decomposition for twoloop amplitudes 160] in $U\left(N_{c}\right)$ Yang-Mills. We show the form for $n$-gluons, then specialise to the five-point case, comparing the $U\left(N_{c}\right)$ and $S U\left(N_{c}\right)$ colour decompositions, to highlight the physical amplitudes present and show the group theory relations used as checks. A general two-loop amplitude may be expanded in a colour trace basis as,

$$
\begin{aligned}
& \mathcal{A}_{n}^{(2)}(1,2, \cdots, n)=N_{c}^{2} \sum_{S_{n} / Z_{n}} \operatorname{Tr}\left(T^{a_{1}} T^{a_{2}} \cdots T^{a_{n}}\right) A_{n: 1}^{(2)}\left(a_{1}, a_{2}, \cdots, a_{n}\right) \\
+ & N_{c} \sum_{r=2}^{[n / 2]+1} \sum_{S_{n} /\left(Z_{r-1} \times Z_{n+1-r}\right)} \operatorname{Tr}\left(T^{a_{1}} T^{a_{2}} \cdots T^{a_{r-1}}\right) \operatorname{Tr}\left(T^{b_{r}} \cdots T^{b_{n}}\right) A_{n: r}^{(2)}\left(a_{1}, a_{2}, \cdots, a_{r-1} ; b_{r} \cdots b_{n}\right) \\
+ & \sum_{s=1}^{[n / 3]} \sum_{t=s}^{[(n-s) / 2]} \sum_{S_{n} /\left(Z_{s} \times Z_{t} \times Z_{n-s-t}\right)} \operatorname{Tr}\left(T^{a_{1}} \cdots T^{a_{s}}\right) \operatorname{Tr}\left(T^{b_{s+1}} \cdots T^{b_{s+t}}\right) \operatorname{Tr}\left(T^{c_{s+t+1}} \cdots T^{c_{n}}\right) \\
+ & \sum_{S_{n} / Z_{n}} \operatorname{Tr}\left(T^{a_{1}} T^{a_{2}} \cdots T_{n: s, t}^{(2)}\left(a_{1}, \cdots, a_{s} ; b_{s+1} \cdots b_{s+t} ; c_{s+t+1} \cdots c_{n}\right)\right.
\end{aligned}
$$




\section{COLOUR DRESSED UNITARITY AND RECURSION FOR YANG-MILLS TWO-LOOP ALL-PLUS AMPLITUDES}

For $n$ even and $r=n / 2+1$ there is an extra $Z_{2}$ in the summation to ensure each colour structure only appears once. In the $s, t$ summations there is an extra $Z_{2}$ when exactly two of $s, t$ and $n-s-t$ are equal and an extra $S_{3}$ when all three are equal. At five-point, the specific scattering configuration in question, this reduces to,

$$
\begin{aligned}
\mathcal{A}_{5}^{(2)}(1,2,3,4,5) & =N_{c}^{2} \sum_{S_{5} / Z_{5}} \operatorname{Tr}\left(T^{a_{1}} T^{a_{2}} T^{a_{3}} T^{a_{4}} T^{a_{5}}\right) A_{5: 1}^{(2)}\left(a_{1}, a_{2}, a_{3}, a_{4}, a_{5}\right) \\
& +N_{c} \sum_{S_{5} / Z_{4}} \operatorname{Tr}\left(T^{a_{1}}\right) \operatorname{Tr}\left(T^{b_{2}} T^{b_{3}} T^{b_{4}} T^{b_{5}}\right) A_{5: 2}^{(2)}\left(a_{1} ; b_{2}, b_{3}, b_{4}, b_{5}\right) \\
& +N_{c} \sum_{S_{5} /\left(Z_{2} \times Z_{3}\right)} \operatorname{Tr}\left(T^{a_{1}} T^{a_{2}}\right) \operatorname{Tr}\left(T^{b_{3}} T^{b_{4}} T^{b_{5}}\right) A_{5: 3}^{(2)}\left(a_{1}, a_{2} ; b_{3}, b_{4}, b_{5}\right) \\
& +\sum_{S_{5} /\left(Z_{2} \times Z_{3}\right)} \operatorname{Tr}\left(T^{a_{1}}\right) \operatorname{Tr}\left(T^{b_{2}}\right) \operatorname{Tr}\left(T^{c_{3}} T^{c_{4}} T^{c_{5}}\right) A_{5: 1,1}^{(2)}\left(a_{1} ; b_{2} ; c_{3}, c_{4}, c_{5}\right) \\
& +\sum_{S_{5} /\left(Z_{2} \times Z_{2} \times Z_{2}\right)} \operatorname{Tr}\left(T^{a_{1}}\right) \operatorname{Tr}\left(T^{b_{2}} T^{b_{3}}\right) \operatorname{Tr}\left(T^{c_{4}} T^{c_{5}}\right) A_{5: 1,2}^{(2)}\left(a_{1} ; b_{2}, b_{3} ; c_{4}, c_{5}\right) \\
& +\sum_{S_{5} / Z_{5}} \operatorname{Tr}\left(T^{a_{1}} T^{a_{2}} T^{a_{3}} T^{a_{4}} T^{a_{5}}\right) A_{5: 1 B}^{(2)}\left(a_{1}, a_{2}, a_{3}, a_{4}, a_{5}\right),
\end{aligned}
$$

which is the full colour amplitude we calculate in this chapter. There are six partial amplitudes that will be directly computed using colour dressed unitarity, but only three are present in the physical $S U\left(N_{c}\right)$ theory. To highlight the physical amplitudes, we present the decomposition in an $S U\left(N_{c}\right)$ gauge group,

$$
\begin{aligned}
\mathcal{A}_{5}^{(2)}(1,2,3,4,5) & =N_{c}^{2} \sum_{S_{5} / Z_{5}} \operatorname{Tr}\left(T^{a_{1}} T^{a_{2}} T^{a_{3}} T^{a_{4}} T^{a_{5}}\right) A_{5: 1}^{(2)}\left(a_{1}, a_{2}, a_{3}, a_{4}, a_{5}\right) \\
& +N_{c} \sum_{S_{5} /\left(Z_{2} \times Z_{3}\right)} \operatorname{Tr}\left(T^{a_{1}} T^{a_{2}}\right) \operatorname{Tr}\left(T^{b_{3}} T^{b_{4}} T^{b_{5}}\right) A_{5: 3}^{(2)}\left(a_{1}, a_{2} ; b_{3}, b_{4}, b_{5}\right) \\
& +\sum_{S_{5} / Z_{5}} \operatorname{Tr}\left(T^{a_{1}} T^{a_{2}} T^{a_{3}} T^{a_{4}} T^{a_{5}}\right) A_{5: 1 B}^{(2)}\left(a_{1}, a_{2}, a_{3}, a_{4}, a_{5}\right) .
\end{aligned}
$$

Thus, there are three independent physical functions to be determined: $A_{5: 1}^{(2)}, A_{5: 3}^{(2)}$ and $A_{5: 1 B}^{(2)}$. Unlike in the one-loop case, the $U(1)$-decoupling identities do not determine any of the three $S U\left(N_{c}\right)$ partial amplitudes alone, however they can 


\subsection{Two-Loop Amplitudes}

be used to obtain the specifically $U\left(N_{c}\right)$ functions, $A_{5: 2}^{(2)}, A_{5: 1,1}^{(2)}$ and $A_{5: 1,2}^{(2)}$ :

$$
\begin{aligned}
A_{5: 2}^{(2)}(1 ; 2,3,4,5) & =-A_{5: 1}^{(2)}(1,2,3,4,5)-A_{5: 1}^{(2)}(2,1,3,4,5)-A_{5: 1}^{(2)}(2,3,1,4,5)-A_{5: 1}^{(2)}(2,3,4,1,5), \\
A_{5: 1,1}^{(2)}(4 ; 5 ; 1,2,3) & =-A_{5: 2}^{(2)}(5 ; 1,2,3,4)-A_{5: 2}^{(2)}(5 ; 1,2,4,3)-A_{5: 2}^{(2)}(5 ; 1,4,2,3)-A_{5: 3}^{(2)}(4,5 ; 1,2,3) \\
& =-A_{5: 3}^{(2)}(4,5 ; 1,2,3)+\sum_{C O P\{4,5\}\{1,2,3\}} A_{5: 1}^{(2)}(1,2,3,4,5)
\end{aligned}
$$

and

$$
\begin{aligned}
A_{5: 1,2}^{(2)}(1 ; 2,3 ; 4,5) & =-A_{5: 3}^{(2)}(2,3 ; 1,4,5)-A_{5: 3}^{(2)}(2,3 ; 1,5,4)-A_{5: 3}^{(2)}(4,5 ; 1,2,3)-A_{5: 3}^{(2)}(4,5 ; 1,3,2) \\
& =0 .
\end{aligned}
$$

The last relation in (4.21) is a simplification arising at five-point for the $A_{5: 1,2}^{(2)}$ result since the flip symmetry of $A_{5: 3}^{(2)}$ implies,

$$
A_{5: 3}^{(2)}(1,2 ; 3,4,5)=-A_{5: 3}^{(2)}(2,1 ; 5,4,3)=-A_{5: 3}^{(2)}(1,2 ; 5,4,3)
$$

i.e. reversed ordering of a two element list is equivalent to cycling the list. This does not extend beyond five-point.

Decoupling identities do not relate the $A_{n: 1 B}^{(2)}$ to the other terms as they do not contribute to the same order in colour once a $U(1)$ leg has been chosen, but do have the property of a tree-like identity,

$$
A_{n: 1 B}^{(2)}(1,2,3, \cdots n)+A_{n: 1 B}^{(2)}(2,1,3, \cdots n)+\cdots A_{n: 1 B}^{(2)}(2, \cdots, 1, n)=0,
$$

which in itself does not specify $A_{n: 1 B}^{(2)}$ uniquely. There are however further colour restrictions beyond the decoupling identities [15, 16] which may be obtained by recursive methods. These, together with eq. (4.23) determine the $A_{5: 1 B}^{(2)}$ in terms 


\section{COLOUR DRESSED UNITARITY AND RECURSION FOR YANG-MILLS TWO-LOOP ALL-PLUS AMPLITUDES}

of the $A_{5: 1}$ and $A_{5: 3}$

$$
\begin{aligned}
A_{5: 1 B}^{(2)}(1,2,3,4,5)= & -A_{5: 1}^{(2)}(1,2,4,3,5)+2 A_{5: 1}^{(2)}(1,2,5,3,4)+A_{5: 1}^{(2)}(1,2,5,4,3) \\
& -A_{5: 1}^{(2)}(1,3,2,4,5)+2 A_{5: 1}^{(2)}(1,3,4,2,5)-5 A_{5: 1}^{(2)}(1,3,5,2,4) \\
& -2 A_{5: 1}^{(2)}(1,3,5,4,2)+2 A_{5: 1}^{(2)}(1,4,2,3,5)+A_{5: 1}^{(2)}(1,4,3,2,5) \\
& +2 A_{5: 1}^{(2)}(1,4,5,2,3)+A_{5: 1}^{(2)}(1,4,5,3,2) \\
- & \frac{1}{2} \sum_{Z_{5}(1,2,3,4,5)}\left(A_{5: 3}^{(2)}(1,2 ; 3,4,5)-A_{5: 3}^{(2)}(1,3 ; 2,4,5)\right) .
\end{aligned}
$$

Colour dressed unitarity determines $A_{5: 1 B}^{(2)}$ directly, but we use 4.24 as a consistency check.

\subsection{Singularity Structure of the Two-Loop All- Plus Amplitudes}

The IR singular structure of a colour partial amplitude was determined by general theorems [33]. This motivates an organisation of the amplitude into terms that are divergent in the dimensional regulator, i.e. for the all-plus amplitude the IR singular terms $U_{n: \lambda}^{(2)}$ and terms that are finite in the dimensional regulator $F_{n: \lambda}^{(2)}$,

$$
A_{n: \lambda}^{(2)}=U_{n: \lambda}^{(2)}+F_{n: \lambda}^{(2)}+\mathcal{O}(\epsilon)
$$

This organisation was found to be the case in the leading in colour context and we show that it is indeed the case at (sub-)subleading orders in the colour as well. The subscript $\lambda$ is used to refer to the particular order in colour. As the all-plus tree amplitude vanishes, $U_{n: \lambda}^{(2)}$ simplifies considerably and is at worst $1 / \epsilon^{2}$. In general an amplitude has UV divergences, collinear IR divergences and soft IR divergences. As the tree amplitude vanishes, both the UV divergences and collinear IR divergences are proportional to $n$ and cancel leaving only the soft IR singular terms $\left[32\right.$. The leading case, $U_{n: 1}^{(2)}$, is proportional to the one-loop amplitude,

$$
U_{n: 1}^{(2)}=A_{n: 1}^{(1)} \times I_{n: 1}^{(2)}
$$


where

$$
I_{n: 1}^{(2)}=\left[-\sum_{i=1}^{n} \frac{1}{\epsilon^{2}}\left(\frac{\mu^{2}}{-s_{i, i+1}}\right)^{\epsilon}\right] .
$$

In Appendix D the form of the two-loop IR divergences for the other unrenormalised partial amplitudes are presented in a colour trace basis.

Given the general expressions for $U_{n: \lambda}^{(2)}$, the challenge is to compute the finite parts of the amplitude: $F_{n: \lambda}^{(2)}$. This finite remainder function $F_{n: \lambda}^{(2)}$ can be further organised into polylogarithmic and rational pieces,

$$
F_{n: \lambda}^{(2)}=P_{n: \lambda}^{(2)}+R_{n: \lambda}^{(2)}
$$

The polylogarithmic terms can be computed using four-dimensional unitarity whereas the rational pieces are calculated via recursive methods.

\subsection{Colour Dressed Unitarity}

We begin the discussion of the cut-constructible pieces of the amplitude by purely considering the kinematics of the two-loop all-plus amplitude. Understanding the kinematic structure of the functions that are at play in this calculation vastly simplifies the amount of work that one needs to perform in the full colour calculation. The discussion on the kinematics of the all-plus amplitude was set out in section 3.2 and section 3.3. We refer the reader to those sections for a more in depth look. We briefly review the integral basis required to compute the two-loop all-plus gluon amplitude to show its full colour extension.

Again, even at full colour, the two-loop cuts effectively become one-loop cuts with the full colour all-plus one-loop amplitude acting as an additional on-shell vertex. The non-vanishing four dimensional cuts are shown in fig. 4.2. 


\section{COLOUR DRESSED UNITARITY AND RECURSION FOR YANG-MILLS TWO-LOOP ALL-PLUS AMPLITUDES}
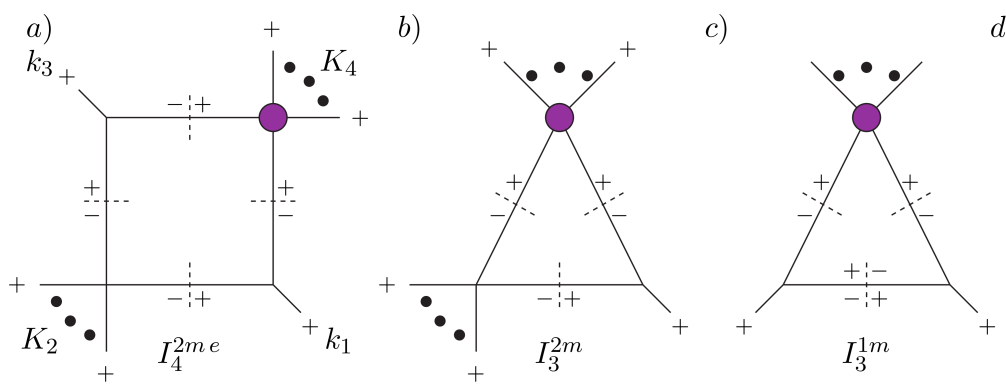

d)

Figure 4.2: Four dimensional cuts of the two-loop all-plus amplitude involving an all-plus one-loop vertex. For the five-gluon amplitude, we take $K_{2} \rightarrow 0$, and as always $K_{4}^{2} \neq 0$.

At five-point, we only have contributions from the one-mass box integral function, and the one- and two-mass triangle integral functions. Catani predicted the absence of $\epsilon^{-1}$ terms even at subleading orders in colour and as such, there are no contributions from bubble integral functions here. The integral functions are given by,

$$
\begin{aligned}
I_{4}^{1 m}\left(S, T, K_{4}^{2}\right)=\frac{2 \mu^{2 \epsilon}}{S T} & {\left[\frac{1}{\epsilon^{2}}\left((-S)^{-\epsilon}+(-T)^{-\epsilon}-\left(-K_{4}^{2}\right)^{-\epsilon}\right)\right.} \\
& \left.\quad-\operatorname{Li}_{2}\left(1-\frac{K_{4}^{2}}{S}\right)-\operatorname{Li}_{2}\left(1-\frac{K_{4}^{2}}{T}\right)-\frac{1}{2} \ln ^{2}\left(\frac{S}{T}\right)-\frac{\pi^{2}}{6}\right]+\mathcal{O}(\epsilon),
\end{aligned}
$$

where $S=\left(k_{1}+K_{2}\right)^{2}$ and $T=\left(k_{1}+K_{4}\right)^{2}$, and we write,

$$
\mathrm{F}^{1 \mathrm{~m}}\left[S, T, K_{4}^{2}\right]=\mathrm{Li}_{2}\left(1-\frac{K_{4}^{2}}{S}\right)+\operatorname{Li}_{2}\left(1-\frac{K_{4}^{2}}{T}\right)+\frac{1}{2} \ln ^{2}\left(\frac{S}{T}\right)+\frac{\pi^{2}}{6} .
$$

The one-mass boxes will completely determine the form of $P_{n: \lambda}^{(2)}$ since they are the source of the finite polylogarithms. The triangle integral functions are given as,

$$
I_{3}^{1 \mathrm{~m}}\left(K_{4}^{2}\right)=\frac{1}{\epsilon^{2}}\left(-K_{4}^{2}\right)^{-1-\epsilon}, \text { and } I_{3}^{2 \mathrm{~m}}\left(K_{4}^{2}, K_{3}^{2}\right)=\frac{1}{\epsilon^{2}} \frac{\left(-K_{4}^{2}\right)^{-\epsilon}-\left(-K_{3}^{2}\right)^{-\epsilon}}{\left(-K_{4}^{2}\right)-\left(-K_{3}^{2}\right)} \text {, }
$$

where $K_{3}^{2}=\left(K_{2}+k_{3}\right)^{2}$ in the labelling of fig. 4.2. This same integral basis, used in the leading case, is easily extended to the full colour case. Generalised unitarity is used with the same philosophy, of cutting the diagrams systematically 


\subsection{Colour Dressed Unitarity}

using quadruple cuts then triple cuts. However, in the full colour case we promote partial amplitudes to full colour amplitudes. Explicitly, the quadruple cut of the one-mass box,

$$
\int \mathrm{dLIPS} A_{4: 1}^{(1)}\left(d^{+}, e^{+}, l_{1}^{+},-l_{4}^{-}\right) A_{3}^{(0)}\left(-l_{1}^{-}, a^{+}, l_{2}^{+}\right) A_{3}^{(0)}\left(-l_{2}^{-}, b^{+}, l_{3}^{-}\right) A_{3}^{(0)}\left(-l_{3}^{+}, c^{+}, l_{4}^{-}\right),
$$

is promoted to

$$
\int \mathrm{dLIPS} \mathcal{A}_{4}^{(1)}\left(d^{+}, e^{+}, l_{1}^{+},-l_{4}^{-}\right) \mathcal{A}_{3}^{(0)}\left(-l_{1}^{-}, a^{+}, l_{2}^{+}\right) \mathcal{A}_{3}^{(0)}\left(-l_{2}^{-}, b^{+}, l_{3}^{-}\right) \mathcal{A}_{3}^{(0)}\left(-l_{3}^{+}, c^{+}, l_{4}^{-}\right) .
$$

The cut integrals are now colour dressed and come accompanied with a variety of trace structures multiplying the partial amplitudes. We consider a basis of diagrams for which we pick an ordering for the external legs in the cuts, as shown in (4.33) for the following reason. When colour dressing a cut integral, amplitudes will contain trace structures that have generators of the loop momenta. These trace structures will be combined, along with their respective partial amplitudes, in the cut integral through application of the $U\left(N_{c}\right)$ colour algebra shown in subsection 2.3.1. This method produces all possible types of trace structures composing the two-loop colour decomposition but not the full set of traces we expect. It is possible to take this generated colour decomposition and sum over each of the independent diagrams that appear in the cuts, once the cut integral has been performed, due to the identical helicity of the external legs. Specifically there are thirty independent one-mass boxes and two-mass triangles, along with ten independent one-mass triangles. The total sum of the cuts then recovers every possible trace present in the full colour two-loop amplitude.

The basis of integrals we consider in the five-point case are contained in the integral basis of fig. 4.2 , with $K_{2}^{2}=0$ and $K_{4}$ is the sum of two external legs.

With this philosophy in mind, it is extremely useful to simplify the tree-level and one-loop amplitudes that will appear in the cut integrands, before computing the cuts. There are two types of tree-level amplitudes entering the cuts, the threeand four-point trees. Since the three-point tree amplitude has two traces that 


\section{COLOUR DRESSED UNITARITY AND RECURSION FOR YANG-MILLS TWO-LOOP ALL-PLUS AMPLITUDES}

are antisymmetric and the kinematic expressions are flip symmetric, each trace structure has a common factor related by a minus sign.

$$
\begin{aligned}
\mathcal{A}_{3}^{(0)} & =\operatorname{Tr}[a b c] A_{3}^{(0)}(a, b, c)+\operatorname{Tr}[a c b] A_{3}^{(0)}(a, c, b) \\
& =A_{3}^{(0)}(a, b, c)(\operatorname{Tr}[a b c]-\operatorname{Tr}[a c b])
\end{aligned}
$$

Whereas the four-point MHV can be factored out as

$$
\begin{aligned}
\mathcal{A}_{4}^{(0)}\left(a^{-}, b^{-}, c^{+}, d^{+}\right)=A_{4}^{(0)}\left(a^{-}, b^{-}, c^{+}, d^{+}\right)((\operatorname{Tr}[a b c d]+\operatorname{Tr}[a d c b]) & +\frac{t}{u}(\operatorname{Tr}[a b d c]+ \\
& \operatorname{Tr}[a c d b]) \\
& \left.+\frac{s}{u}(\operatorname{Tr}[a c b d]+\operatorname{Tr}[a d b c])\right) .
\end{aligned}
$$

The one-loop four-point all-plus enters into the cut expressions of both the 1-mass box and 2-mass triangle. Due to four-point kinematics, the partial amplitudes are related via the following

$$
A_{4: 1}^{(1)}(a, b, c, d)=A_{4: 1}^{(1)}(a, b, d, c)=A_{4: 1}^{(1)}(a, c, b, d),
$$

which implies that

$$
A_{4: 2}^{(1)}(a ; b, c, d)=-3 A_{4: 1}^{(1)}(a, b, c, d) \text { and } A_{4: 3}^{(1)}(a, b ; c, d)=6 A_{4: 1}^{(1)}(a, b, c, d) .
$$

As each subleading partial amplitude is proportional to the leading in colour amplitude, there is a common kinematic factor so that the full colour amplitude 


\subsection{Colour Dressed Unitarity}

factorises into a colour component and kinematic component. In full this is,

$$
\begin{aligned}
& \mathcal{A}_{4}^{(1)}\left(a^{+}, b^{+}, c^{+}, d^{+}\right)=A_{4: 1}^{(1)}\left(a^{+}, b^{+}, c^{+}, d^{+}\right) \\
& \times\left[N_{c}(\operatorname{Tr}[a b c d]+\operatorname{Tr}[a d c b]+\operatorname{Tr}[a c b d]+\operatorname{Tr}[a d b c]+\operatorname{Tr}[a c d b]+\operatorname{Tr}[a b d c])\right. \\
& \quad-3(\operatorname{Tr}[a](\operatorname{Tr}[b c d]-\operatorname{Tr}[d c b])+\operatorname{Tr}[b](\operatorname{Tr}[a c d]-\operatorname{Tr}[d c a]) \\
& \quad+\operatorname{Tr}[c](\operatorname{Tr}[a b d]-\operatorname{Tr}[d b a])+\operatorname{Tr}[d](\operatorname{Tr}[a b c]-\operatorname{Tr}[c b a])) \\
& +6(\operatorname{Tr}[a b] \operatorname{Tr}[c d]+\operatorname{Tr}[a c] \operatorname{Tr}[b d]+\operatorname{Tr}[a d] \operatorname{Tr}[b c])]
\end{aligned}
$$

We abbreviate it to the following for ease,

$$
\mathcal{A}_{4}^{(1)}\left(\ell_{1}, \ell_{4}, d, e\right)=\mathcal{C} \times A_{4: 1}^{(1)}\left(\ell_{4}, d, e, \ell_{1}\right)
$$

The only other one-loop amplitude to enter into the cuts is the five-point all-plus. It is not possible in this case to pull out a common factor from the full colour decomposition and we retain its form for the calculation. However as will be seen later, it is possible to relate the cut integrals for which the five-point all-plus appears in at different orders of the colour to three basis integrals.

We now turn to consider the three non-zero basis diagrams contributing to the cuts, beginning with the one-mass box. The colour and kinematics can be factored out completely to give a full colour cut integral

$$
C u t_{b o x}=\mathfrak{C}^{\prime} \times A_{3}^{(0)}\left(a, \ell_{2}, \ell_{1}\right) A_{3}^{(0)}\left(b, \ell_{3}, \ell_{2}\right) A_{3}^{(0)}\left(c, \ell_{4}, \ell_{3}\right) A_{4: 1}^{(1)}\left(\ell_{4}, d, e, \ell_{1}\right) .
$$

The colour structures can be shown diagrammatically as in fig. 4.3. Given the configuration of external legs and colour labelling of the internal legs shown in fig. 4.4, the combination of trace structures arising from the four components of 


\section{COLOUR DRESSED UNITARITY AND RECURSION FOR YANG-MILLS TWO-LOOP ALL-PLUS AMPLITUDES}
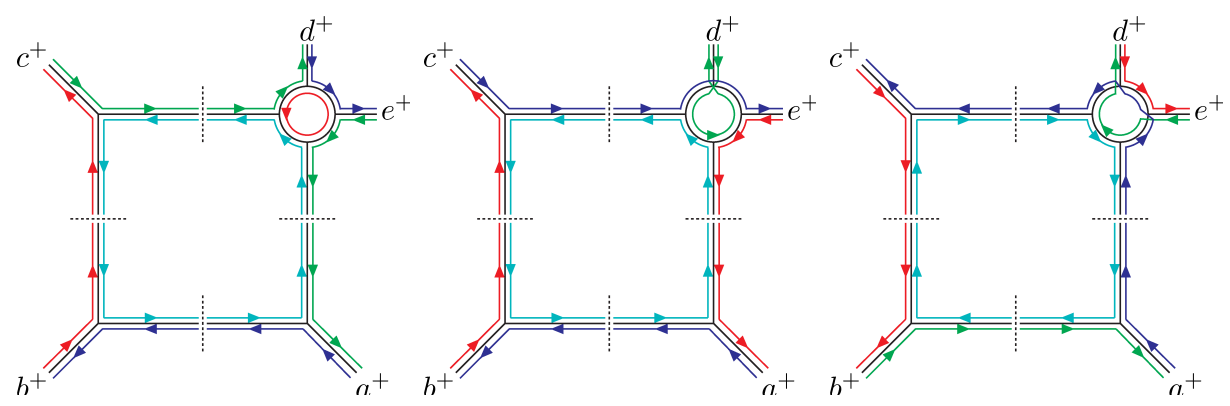

Figure 4.3: Four-dimensional quadruple cuts uniquely define the coefficients of the box integral functions. Extended to a full colour amplitude, the cut diagrams appear as three types of boxes reflecting the three types of colour structures contained in the full colour four-point all-plus one-loop amplitude.

the cut gives explicitly,

$$
\begin{aligned}
& \mathfrak{C}_{(d e ; a b c)}^{\prime}=\sum_{m, n, p, q}((\operatorname{Tr}[a m n]-\operatorname{Tr}[m a n])(\operatorname{Tr}[b p n]-\operatorname{Tr}[p b n])(\operatorname{Tr}[p c q]-\operatorname{Tr}[p q c])) \\
& \times\left(N_{c} \operatorname{Tr}[\text { mqed }]+N_{c} \operatorname{Tr}[\text { meqd }] / 2+N_{c} \operatorname{Tr}[\text { qemd }] / 2+N_{c} \operatorname{Tr}[\text { qmed }]\right. \\
& -3 \operatorname{Tr}[m] \operatorname{Tr}[q d e]-3 \operatorname{Tr}[q] \operatorname{Tr}[m d e]-3 \operatorname{Tr}[d] \operatorname{Tr}[e m q]-3 \operatorname{Tr}[d] \operatorname{Tr}[e q m] \\
& +3 \operatorname{Tr}[d e] \operatorname{Tr}[m q]+3 \operatorname{Tr}[d m] \operatorname{Tr}[e q]+3 \operatorname{Tr}[d q] \operatorname{Tr}[e m]+\{d \leftrightarrow e\}) \\
& =N_{c}^{2}(\operatorname{Tr}[d e a b c]+\operatorname{Tr}[e d a b c]-\operatorname{Tr}[b a d e c]-\operatorname{Tr}[b a e d c]) \\
& +N_{c}(-2 \operatorname{Tr}[d](\operatorname{Tr}[e a b c]-\operatorname{Tr}[b a e c])-2 \operatorname{Tr}[e](\operatorname{Tr}[d a b c]-\operatorname{Tr}[b a d c]) \\
& -\operatorname{Tr}[a](\operatorname{Tr}[d e b c]+\operatorname{Tr}[e d b c]-\operatorname{Tr}[b d e c]-\operatorname{Tr}[b e d c]) \\
& -\operatorname{Tr}[b](\operatorname{Tr}[d e a c]+\operatorname{Tr}[e d a c]-\operatorname{Tr}[a e d c]-\operatorname{Tr}[a d e c]) \\
& -\operatorname{Tr}[c](\operatorname{Tr}[d e a b]+\operatorname{Tr}[e d a b]-\operatorname{Tr}[a d e b]-\operatorname{Tr}[a e d b]) \\
& +8 \operatorname{Tr}[d e](\operatorname{Tr}[a b c]-\operatorname{Tr}[b a c])+\operatorname{Tr}[d a](\operatorname{Tr}[b e c]-\operatorname{Tr}[e b c]) \\
& +\operatorname{Tr}[d b](\operatorname{Tr}[a e c]-\operatorname{Tr}[e a c])+\operatorname{Tr}[d c](\operatorname{Tr}[a e b]-\operatorname{Tr}[e a b]) \\
& -\operatorname{Tr}[e a](\operatorname{Tr}[d b c]-\operatorname{Tr}[b d c])-\operatorname{Tr}[e b](\operatorname{Tr}[d a c]-\operatorname{Tr}[a d c])-\operatorname{Tr}[e c](\operatorname{Tr}[d a b]-\operatorname{Tr}[a d b])) \\
& +3(-2 \operatorname{Tr}[d] \operatorname{Tr}[e](\operatorname{Tr}[a b c]-\operatorname{Tr}[b a c])+\operatorname{Tr}[d] \operatorname{Tr}[a](\operatorname{Tr}[e b c]-\operatorname{Tr}[b e c]) \\
& +\operatorname{Tr}[d] \operatorname{Tr}[b](\operatorname{Tr}[e a c]-\operatorname{Tr}[a e c])+\operatorname{Tr}[d] \operatorname{Tr}[c](\operatorname{Tr}[e a b]-\operatorname{Tr}[a e b]) \\
& +\operatorname{Tr}[e] \operatorname{Tr}[a](\operatorname{Tr}[d b c]-\operatorname{Tr}[b d c])+\operatorname{Tr}[e] \operatorname{Tr}[b](\operatorname{Tr}[d a c]-\operatorname{Tr}[a d c]) \\
& +\operatorname{Tr}[e] \operatorname{Tr}[c](\operatorname{Tr}[d a b]-\operatorname{Tr}[a d b])) \\
& +6(\operatorname{Tr}[\text { deabc }]-\operatorname{Tr}[\text { dcbae }]+\operatorname{Tr}[\text { dcbea }]-\operatorname{Tr}[\text { daebc }]+\operatorname{Tr}[\text { dceba }]-\operatorname{Tr}[\text { dabec }]+\operatorname{Tr}[\text { dcaeb }]-\operatorname{Tr}[\text { dbeac }] \\
& +\operatorname{Tr}[\text { dbaec }]-\operatorname{Tr}[\text { dceab }]+\operatorname{Tr}[\text { dabce }]-\operatorname{Tr}[\text { decba }]+\operatorname{Tr}[\text { daecb }]-\operatorname{Tr}[\text { dbcea }]+\operatorname{Tr}[\text { dbeca }]-\operatorname{Tr}[\text { daceb }]) .
\end{aligned}
$$




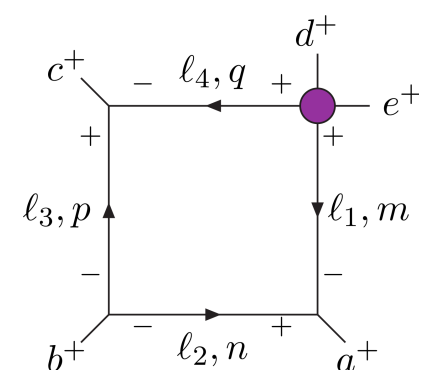

Figure 4.4: The box diagram considered in the quadruple cut with explicit colour labels and internal helicity configuration.

For simplicity, we say this is an expansion of the form,

$$
C_{(d e ; a b c)}^{\prime}=\sum_{\lambda} a_{(d e ; a b c)}^{\lambda} C^{\lambda}
$$

where the $C^{\lambda}$ are the different colour structures.

On the kinematic side, the loop momenta integral is completely constrained by the cuts. The solution to the quadruple cuts in this case is

$$
\begin{aligned}
\ell_{2} & =-\frac{\langle b a\rangle}{\langle c a\rangle} \bar{\lambda}_{b} \lambda_{c}, & \ell_{3} & =-\frac{\langle b c\rangle}{\langle a c\rangle} \bar{\lambda}_{b} \lambda_{a} \\
\ell_{1} & =\frac{\langle a c\rangle \bar{\lambda}_{a}+\langle b c\rangle \bar{\lambda}_{b}}{\langle a c\rangle} \lambda_{a}, & \ell_{4} & =\frac{\langle c a\rangle \bar{\lambda}_{c}+\langle b a\rangle \bar{\lambda}_{b}}{\langle c a\rangle} \lambda_{c} .
\end{aligned}
$$

This solution gives the box integral coefficient as,

$$
\begin{aligned}
A_{3}^{(0)}\left(a, \ell_{2}, \ell_{1}\right) A_{3}^{(0)}\left(b, \ell_{3}, \ell_{2}\right) A_{3}^{(0)}\left(c, \ell_{4}, \ell_{3}\right) A_{4: 1}^{(1)}\left(\ell_{4}, d, e, \ell_{1}\right) & =\frac{2 i}{3} s_{a b} s_{b c} \times \frac{[d e]^{2}}{\langle a b\rangle\langle b c\rangle\langle c a\rangle} \\
& =\frac{1}{3} s_{a b} s_{b c} \times A_{5: 3}^{(1)}(d, e ; a, b, c) .
\end{aligned}
$$

The box integral function contains IR singular terms and finite polylogarithmic terms. We relegate the explicit forms of the IR singular terms to Appendix D. The full contribution from the box functions comes from summing the full colour and kinematic result over the thirty independent diagrams related by relabelling 


\section{COLOUR DRESSED UNITARITY AND RECURSION FOR YANG-MILLS TWO-LOOP ALL-PLUS AMPLITUDES}

of the external legs, i.e

$$
\sum_{S_{5} /\left(Z_{2} \times Z_{2}\right)} \frac{s_{a b} s_{b c}}{3} A_{5: 3}^{(1)}(d, e ; a, b, c) I_{4}^{1 m}\left(s_{a b}, s_{b c}, K_{d e}^{2}\right) .
$$

This is a combination of IR singular terms and polylogarithmic terms. The full sum over one-mass boxes completely determines the leading and subleading in colour polylogarithmic terms. Consequently, the polylogarithmic part of the partial amplitudes is proportional to the box coefficient,

$$
P_{5: \lambda}^{(2)} \propto \sum_{S_{5} /\left(Z_{2} \times Z_{2}\right)} A_{5: 3}(d, e ; a, b, c) \times \mathrm{F}_{a b c ; d e}^{1 m},
$$

where $\mathrm{F}_{a b c ; d e}^{1 m} \equiv \mathrm{F}^{1 m}\left[s_{a b}, s_{b c}, s_{d e}\right]$. Specifically we recover the previous results of $[96$ and [110]. Defining $S_{5: 1}=Z_{5}(a, b, c, d, e), S_{5: 2}=Z_{4}(b, c, d, e)$ and $S_{5: 3}=Z_{2}(a, b) \times$ $Z_{3}(c, d, e)$ we have,

$$
\begin{aligned}
P_{5: 1}^{(2)}(a, b, c, d, e) & =\sum_{S_{5: 1}}-A_{5: 3}^{(1)}(d, e ; a, b, c) \mathrm{F}_{a b c ; d e}^{1 m} \\
P_{5: 3}^{(2)}(a, b: c, d, e) & =\frac{4}{3} \sum_{S_{5: 3}}\left(A_{5: 3}^{(1)}(a, b ; c, d, e) \mathrm{F}_{c d e, a b}^{1 m}\right. \\
& \left.+\frac{1}{4} A_{5: 3}^{(1)}(a, c ; b, e, d)\left(\mathrm{F}_{b e d ; a c}^{1 m}+\mathrm{F}_{b d e ; a c}^{1 m}-\mathrm{F}_{d b e ; a c}^{1 m}\right)\right)
\end{aligned}
$$

We also directly determine the polylogarithmic part of the sub-subleading $S U\left(N_{c}\right)$ partial amplitude,

$$
\begin{aligned}
P_{5: 1 B}^{(2)}(a, b, c, d, e)=2 \sum_{S_{5: 1}} & \left(A_{5: 3}^{(1)}(a, b ; c, d, e) \mathrm{F}_{c d e ; a b}^{1 m}+\right. \\
& \left.A_{5: 3}^{(1)}(a, c ; b, e, d)\left(\mathrm{F}_{b e d ; a c}^{1 m}+\mathrm{F}_{b d e ; a c}^{1 m}-\mathrm{F}_{d b e ; a c}^{1 m}\right)\right) .
\end{aligned}
$$

This expression satisfies the constraint obtained by using the results of (4.49) in (4.24). 


\subsection{Colour Dressed Unitarity}

The specifically $U\left(N_{c}\right)$ partial amplitudes may also be extracted directly:

$$
\begin{aligned}
P_{5: 2}^{(2)}(a ; b, c, d, e)=-\frac{2}{3} \sum_{S_{5: 2}}( & A_{5: 3}^{(1)}(a, b ; c, d, e) \mathrm{F}_{c d e ; a b}^{1 m} \\
& \left.+\frac{1}{2} A_{5: 3}^{(1)}(b, c ; a, e, d)\left(\mathrm{F}_{a d e ; b c}^{1 m}+\mathrm{F}_{d e a ; b c}^{1 m}-\mathrm{F}_{d a e ; b c}^{1 m}\right)\right)
\end{aligned}
$$

and

$$
\begin{aligned}
P_{5: 1,1}^{(2)}(a ; b ; c, d, e)=-\sum_{S_{5: 3}} & \left(A_{5: 3}^{(1)}(a, b ; c, d, e) \mathrm{F}_{c d e ; a b}^{1 m}\right. \\
& \left.+A_{5: 3}^{(1)}(a, c ; b, e, d)\left(\mathrm{F}_{b e d ; a c}^{1 m}+\mathrm{F}_{b d e ; a c}^{1 m}-\mathrm{F}_{d b e ; a c}^{1 m}\right)\right) .
\end{aligned}
$$

As a check we have confirmed that these satisfy the decoupling identities (4.21).

The remaining one- and two-mass triangles only contribute to the IR singular term. Since the IR terms have been determined in general, we include this calculation as a proof of concept of the method. The two-mass triangle cut integral is given by,

$$
c_{2 m}=\int d \operatorname{LIPS} \mathcal{A}_{4}^{(1)}\left(d^{+}, e^{+}, l_{1}^{+},-l_{3}^{+}\right) \mathcal{A}_{3}^{(0)}\left(-l_{1}^{-}, a^{+}, l_{2}^{+}\right) \mathcal{A}_{4}^{(0)}\left(l_{3}^{-},-l_{2}^{-}, b^{+}, c^{+}\right) .
$$

There is a common term that can be factored out of $\mathcal{A}_{4}^{(1)}$ and the $\mathcal{A}_{3}^{(0)}$, however the $\mathcal{A}_{4}^{(0)}$ is not so trivial. In this configuration, if we factor out the first term in the full colour amplitude we get,

$$
\begin{aligned}
& \mathcal{A}_{4}^{(0)}\left(l_{3}^{-},-l_{2}^{-}, b^{+}, c^{+}\right)=A_{4: 1}^{(0)}\left(l_{3}^{-},-l_{2}^{-}, b^{+}, c^{+}\right)\left(\left(\operatorname{Tr}\left[l_{3} l_{2} b c\right]+\operatorname{Tr}\left[l_{3} c b l_{2}\right]\right)\right. \\
& \left.\quad+\frac{\left(l_{3}+k_{c}\right)^{2}}{\left(l_{3}+k_{b}\right)^{2}}\left(\operatorname{Tr}\left[l_{3} l_{2} c b\right]+\operatorname{Tr}\left[l_{3} b c l_{2}\right]\right)+\frac{s_{b c}}{\left(l_{3}+k_{b}\right)^{2}}\left(\operatorname{Tr}\left[l_{3} b l_{2} c\right]+\operatorname{Tr}\left[l_{3} c l_{2} b\right]\right)\right)
\end{aligned}
$$

By considering the power counting of the factored rational function in the cut integral multiplying the colour bracket above, we can determine whether we need 


\section{COLOUR DRESSED UNITARITY AND RECURSION FOR YANG-MILLS TWO-LOOP ALL-PLUS AMPLITUDES}

to integrate all three terms. Introducing the colour factor with kinematic terms,

$$
\begin{aligned}
c_{2 m} & =\frac{i}{3} \mathrm{C}_{2 m}^{\prime} \frac{[d e]^{2}[b c]}{\langle a b\rangle\langle b c\rangle} \int d L I P S\left(\frac{\left[a\left|l_{2}\right| b\right\rangle}{\left[c\left|l_{3}\right| c\right\rangle}\left(\operatorname{Tr}\left[l_{3} l_{2} b c\right]+\operatorname{Tr}\left[l_{3} c b l_{2}\right]\right)\right. \\
& \left.+\frac{\left[a\left|l_{2}\right| b\right\rangle}{\left(l_{3}+k_{b}\right)^{2}}\left(\operatorname{Tr}\left[l_{3} l_{2} c b\right]+\operatorname{Tr}\left[l_{3} b c l_{2}\right]\right)+\frac{s_{b c}\left[a\left|l_{2}\right| b\right\rangle}{\left(l_{3}+k_{b}\right)^{2}\left(l_{3}+k_{c}\right)^{2}}\left(\operatorname{Tr}\left[l_{3} b l_{2} c\right]+\operatorname{Tr}\left[l_{3} c l_{2} b\right]\right)\right) .
\end{aligned}
$$

The first two terms give boxes and triangles while the last term is a linear pentagon integral that will give scalar boxes so we can ignore it since it has already been captured by the quadruple cuts. Dropping the last term and focussing on the former,

$$
\begin{aligned}
c_{2 m}=\frac{i}{3} \mathrm{C}_{2 m}^{\prime} \frac{[d e]^{2}[b c]}{\langle a b\rangle\langle b c\rangle} \int d \operatorname{LIPS}( & \frac{\left[a\left|l_{2}\right| b\right\rangle}{\left[c\left|l_{3}\right| c\right\rangle}\left(\operatorname{Tr}\left[l_{3} l_{2} b c\right]+\operatorname{Tr}\left[l_{3} c b l_{2}\right]\right) \\
& \left.+\frac{\left[a\left|l_{2}\right| b\right\rangle}{\left(l_{3}+k_{b}\right)^{2}}\left(\operatorname{Tr}\left[l_{3} l_{2} c b\right]+\operatorname{Tr}\left[l_{3} b c l_{2}\right]\right)\right)
\end{aligned}
$$

Using $\left[c\left|l_{3}\right| c\right\rangle=\left(l_{3}+k_{c}\right)^{2}=\left(l_{2}-k_{b}\right)^{2}=-\left[b\left|l_{2}\right| b\right\rangle$, and $l_{2}=l_{3}+k_{b}+k_{c}$,

$$
\begin{aligned}
\frac{\left[a\left|l_{2}\right| b\right\rangle}{\left[c\left|l_{3}\right| c\right\rangle} & =-\frac{\left[a l_{2}\right]}{\left[b l_{2}\right]} \\
\frac{\left[a\left|l_{2}\right| b\right\rangle}{\left(l_{3}+k_{b}\right)^{2}} & =\frac{\left[a\left|l_{3}+k_{c}\right| b\right\rangle}{\left[b\left|l_{3}\right| b\right\rangle}=\frac{\left[a l_{3}\right]}{\left[b l_{3}\right]}+\frac{[a|c| b\rangle}{\left[b\left|l_{3}\right| b\right\rangle} .
\end{aligned}
$$

We are left with linear boxes that are in a canonical form and a scalar box, which we can discard. The relevant pieces of the cut integral are,

$$
\begin{aligned}
c_{2 m}=\frac{i}{3} \mathrm{C}_{2 m}^{\prime} \frac{[d e]^{2}[b c]}{\langle a b\rangle\langle b c\rangle} \int d L I P S( & -\frac{\left[a l_{2}\right]}{\left[b l_{2}\right]}\left(\operatorname{Tr}\left[l_{3} l_{2} b c\right]+\operatorname{Tr}\left[l_{3} c b l_{2}\right]\right) \\
+ & \left.\frac{\left[a l_{3}\right]}{\left[b l_{3}\right]}\left(\operatorname{Tr}\left[l_{3} l_{2} c b\right]+\operatorname{Tr}\left[l_{3} b c l_{2}\right]\right)\right)
\end{aligned}
$$

The relevant canonical form for a two-mass triangle comes from the general 3mass triangle canonical forms and taking the massless limit for one of the corners. For a linear box we have,

$\varepsilon_{1}=\frac{[Y \ell]}{[X \ell]} \rightarrow E_{1}=\frac{1}{2} \frac{\left[X\left|\left[K_{1}, K_{3}\right]\right| Y\right]}{\left[X\left|K_{1} K_{3}\right| X\right]}=\frac{1}{2} \frac{\left[X\left|\left[K_{1}, K_{2}\right]\right| Y\right]}{\left[X\left|K_{1} K_{2}\right| X\right]}=\frac{1}{2} \frac{\left[X\left|\left[K_{2}, K_{3}\right]\right| Y\right]}{\left[X\left|K_{2} K_{3}\right| X\right]}$ 


\subsection{Colour Dressed Unitarity}

where $K_{1}=k_{d}+k_{e}, K_{2}=k_{a}$ and $K_{3}=k_{b}+k_{c}$ for the two mass-triangle configuration. Performing the cut integral using canonical forms treats the $\ell_{2}$ and $\ell_{3}$ in the same way. Since the functions are identical after integration, we can factor this out keeping track of minus signs in the colour factors and absorbing the overall minus sign from the previous definition of $c_{2 m}$.

$$
c_{2 m}=\mathcal{C}_{2 m}^{\prime}\left(\left(\operatorname{Tr}\left[l_{3} l_{2} b c\right]+\operatorname{Tr}\left[l_{3} c b l_{2}\right]\right)-\left(\operatorname{Tr}\left[l_{3} l_{2} c b\right]+\operatorname{Tr}\left[l_{3} b c l_{2}\right]\right)\right)\left(\frac{-i}{6}\right) \frac{[d e]^{2}\left[a\left|P_{b c}\right| a\right\rangle}{\langle a b\rangle\langle b c\rangle\langle c a\rangle} I_{3}^{2 m}\left[K_{d e}^{2}, K_{b c}^{2}\right]
$$

Summing over the thirty possible 2-mass triangles gives the full contribution from these types of diagrams.

The remaining 1-mass triangle requires a little more work since we can't factor out a common term from the $\mathcal{A}_{5}^{(1)}$ amplitude present in the cuts. The full colour cut integral is given by,

$$
c_{1 m}=\int d \operatorname{LIPS} \mathcal{A}_{5}^{(1)}\left(a^{+}, b^{+}, c^{+}, l_{1}^{+},-l_{3}^{+}\right) \mathcal{A}_{3}^{(0)}\left(-l_{1}^{-}, d^{+}, l_{2}^{ \pm}\right) \mathcal{A}_{3}^{(0)}\left(-l_{2}^{\mp}, e^{+}, l_{3}^{-}\right) .
$$

The tree factors will factorise out and for one helicity configuration are given by,

$$
\begin{aligned}
\mathcal{A}_{3}^{(0)}\left(-l_{1}^{-}, d^{+}, l_{2}^{+}\right) \mathcal{A}_{3}^{(0)}\left(-l_{2}^{-}, e^{+}, l_{3}^{-}\right)= & \frac{\left[l_{2} d\right]^{3}}{\left[d l_{1}\right]\left[l_{1} l_{2}\right]} \frac{\left\langle l_{3} l_{2}\right\rangle^{3}}{\left\langle l_{2} e\right\rangle\left\langle e l_{3}\right\rangle}\left(\operatorname{Tr}\left[d l_{2} l_{1}\right]-\operatorname{Tr}\left[l_{1} l_{2} d\right]\right) \\
& \times\left(\operatorname{Tr}\left[l_{3} l_{2} e\right]-\operatorname{Tr}\left[e l_{2} l_{3}\right]\right) \\
= & \frac{[d e]}{\langle d e\rangle}\left\langle l_{1} l_{3}\right\rangle^{2}\left(\operatorname{Tr}\left[d l_{2} l_{1}\right]-\operatorname{Tr}\left[l_{1} l_{2} d\right]\right)\left(\operatorname{Tr}\left[l_{3} l_{2} e\right]-\operatorname{Tr}\left[e l_{2} l_{3}\right]\right)
\end{aligned}
$$

Note that there are two helicity configurations for the triple cut of the onemass triangle. In the triple cut, the one-loop amplitude is always the same, $\mathcal{A}_{5}^{(1)}\left(a^{+}, b^{+}, c^{+}, l_{1}^{+},-l_{3}^{+},\right)$. However, for configuration $A$ we have the product of trees $\mathcal{A}_{3}^{(0)}\left(d^{+}, l_{2}^{+},-l_{1}^{-}\right) \mathcal{A}_{3}^{(0)}\left(l_{3}^{-},-l_{2}^{-}, e^{+}\right)$, and for configuration $B$ we have $\mathcal{A}_{3}^{(0)}\left(l_{2}^{-},-l_{1}^{-}, d^{+}\right) \mathcal{A}_{3}^{(0)}\left(-l_{2}^{+}, e^{+}, l_{3}^{-}\right)$.

The product of trees for configuration $A$ has been calculated above, and configuration $B$ gives the same result since the two configurations of the trees are 


\section{COLOUR DRESSED UNITARITY AND RECURSION FOR YANG-MILLS TWO-LOOP ALL-PLUS AMPLITUDES}

related by $d \Longleftrightarrow e$ whilst being symmetric under the exchange of those legs. Hence we may sum the two helicity configurations by doubling the result from configuration $A$.

Naively, there are thirty seven separate integrals to perform for the full colour expression of the one-mass triangle. On closer inspection, there are a set of three independent integrals that can be related to the other integrals, and evaluate to functions of the form,

$$
\begin{aligned}
c_{1 m}^{a}[\{a, b, c\}, d, e]=\frac{i}{6} & \frac{s_{d e}}{\langle a b\rangle\langle b c\rangle\langle e d\rangle}\left\{s_{a b}\left(\frac{[a e]}{\langle c d\rangle}-\frac{[a d]}{\langle c e\rangle}\right)-s_{b c}\left(\frac{[c e]}{\langle a d\rangle}-\frac{[c d]}{\langle a e\rangle}\right)-2[a c][d e]\right\}, \\
c_{1 m}^{b}[\{a, b, c\}, d, e] & =\frac{i}{6} \frac{s_{d e}}{\langle b c\rangle}\left\{\frac{[a e]^{2}}{\langle b d\rangle\langle c d\rangle}+\frac{[a d]^{2}}{\langle b e\rangle\langle c e\rangle}\right\} \\
& =-\frac{1}{12} s_{d e}\left\{A_{5: 3}^{(1)}(a, e ; b, c, d)+A_{5: 3}^{(1)}(a, d ; b, c, e)\right\}
\end{aligned}
$$

and

$$
c_{1 m}^{c}[\{a, b, c\}, d, e]=-2 i \frac{s_{d e}^{3}}{\langle a b\rangle\langle b c\rangle\langle c a\rangle\langle d e\rangle^{2}}=-s_{d e} A_{5: 3}^{(1)}(d, e ; a, b, c) .
$$

Rewriting the thirty seven integrals as relabelled versions of the three basis integrals and summing over the ten independent diagrams gives the full contribution from the one-mass triangles.

Combining the IR pieces from the boxes and triangles then gives the correct IR singularities [33, 111] to all orders in colour for the two-loop all-plus

$$
\left(\left.\sum \alpha_{i} I_{4, i}^{2 \mathrm{me}}\right|_{I R}+\sum \alpha_{i} I_{3, i}^{2 \mathrm{~m}}+\sum \alpha_{i} I_{3, i}^{1 \mathrm{~m}}\right)_{\lambda}=U_{n: \lambda}^{(1), \epsilon^{0}}\left(a^{+}, b^{+}, \cdots, n^{+}\right)
$$

where $U_{n: \lambda}^{(2), \epsilon^{0}}\left(1^{+}, 2^{+}, \cdots, n^{+}\right)$is the order $\epsilon^{0}$ truncation. We have checked the relation of (4.58) by using four dimensional unitarity techniques to compute the coefficients and then comparing to the expected form of $U_{n}^{(2)}$ given in appendix D for $n \leq 9$. The coefficients of these IR pieces also satisfy decoupling identities. 


\subsection{Recursion}

The remaining parts of the amplitude are the rational functions $R_{n: \lambda}^{(2)}$ belonging to partial amplitudes contained in the full colour decomposition of the two-loop all-plus. Since we know there are double poles present in the amplitude we apply augmented recursion to tame the double poles and determine the full pole structure directly. In subsection 2.10.1 we described a technique for evaluating the double pole structure of a leading in colour two-loop five-point amplitude. In chapter 3 we showed the extension of this work in the context of calculating the whole rational piece of a leading in colour six- and seven-point amplitude based on work in [88. We briefly review the most relevant aspects of this type of calculation before moving to describe the extensions necessary to determine the full-colour rational piece, but refer the reader back to the previously mentioned chapters that offer more detail on the specifics.

As $R_{n: \lambda}^{(2)}$ is a rational function we can obtain it recursively given sufficient information about its singularities. Introducing a complex parameter, $z$, whilst preserving overall momentum conservation and keeping all external momenta null can be achieved with the aid of the Risager shift [37],

$$
\begin{aligned}
\lambda_{a} \rightarrow \lambda_{\hat{a}} & =\lambda_{a}+z[b c] \lambda_{\eta}, \\
\lambda_{b} \rightarrow \lambda_{\hat{b}} & =\lambda_{b}+z[c a] \lambda_{\eta}, \\
\lambda_{c} \rightarrow \lambda_{\hat{c}} & =\lambda_{c}+z[a b] \lambda_{\eta},
\end{aligned}
$$

where $\lambda_{\eta}$ must satisfy $\langle a \eta\rangle \neq 0$ etc., but is otherwise unconstrained. All-plus amplitudes are well behaved for $|z| \rightarrow 0$ and vanish as required under this shift, allowing the use of Cauchy's theorem to determine the rational piece by computing the residues of the shifted amplitude,

$$
R=R(0)=-\left.\sum_{z_{j} \neq 0} \operatorname{Res}\left[\frac{R(z)}{z}\right]\right|_{z_{j}} .
$$

The Risager shift (4.59) excites poles corresponding to tree to two-loop and oneloop to one-loop factorisations. The former involve only single poles and their contributions arise from the contribution of the rational part of the four-point all- 


\section{COLOUR DRESSED UNITARITY AND RECURSION FOR YANG-MILLS TWO-LOOP ALL-PLUS AMPLITUDES}

plus amplitude [92] and a googly three-point tree. This is the only non-vanishing helicity configuration of the propagator legs since all external legs are positive. The full colour $S U\left(N_{c}\right)$ parts of the two-loop four-point all-plus are given by [70],

$$
\begin{aligned}
& R_{4: 1}^{(2)}\left(K^{+}, c^{+}, d^{+}, e^{+}\right)=\frac{1}{3} A_{4: 1}^{(1)}\left(K^{+}, c^{+}, d^{+}, e^{+}\right)\left(\frac{s_{c e}^{2}}{s_{c d} s_{d e}}+8\right) \\
& R_{4: 3}^{(2)}\left(K^{+}, c^{+} ; d^{+}, e^{+}\right)=\frac{1}{9} A_{4: 3}^{(1)}\left(K^{+}, c^{+} ; d^{+}, e^{+}\right)\left(\frac{s_{c d}^{2}}{s_{c e} s_{d e}}+\frac{s_{c e}^{2}}{s_{c d} s_{d e}}+\frac{s_{d e}^{2}}{s_{c d} s_{c e}}+24\right) .
\end{aligned}
$$

The $U\left(N_{c}\right)$ amplitudes can be determined from these via decoupling identities,

$$
R_{4: 2}^{(2)}\left(K^{+} ; c^{+}, d^{+}, e^{+}\right)=\frac{1}{9} A_{4: 2}^{(1)}\left(K^{+} ; c^{+}, d^{+}, e^{+}\right)\left(\frac{s_{c d}^{2}}{s_{c e} s_{d e}}+\frac{s_{c e}^{2}}{s_{c d} s_{d e}}+\frac{s_{d e}^{2}}{s_{c d} s_{c e}}+24\right)
$$

and

$$
R_{4: 1,1}^{(2)}\left(K^{+} ; c^{+} ; d^{+}, e^{+}\right)=0
$$

A priori, it is not obvious which of the factorisations are required for the correct contribution to the tree to two-loop factorisations at subleading in colour. Colour dressing the two-loop rational amplitude and the corresponding tree ${ }^{1}$,

$$
\mathcal{A}_{3}^{(0)}\left(\hat{a}^{+}, \hat{b}^{+}, \hat{K}^{-}\right) \frac{-1}{s_{a b}} \mathcal{R}_{4}^{(2)}\left(\hat{K}^{+}, \hat{c}^{+}, d^{+}, e^{+}\right)
$$

and combining traces, produces the full contribution to the rational piece from this channel. Additionally, we must sum over all excited channels, for which there are nine distinct combinations of excited poles. Explicitly, they are $s_{a b}, s_{b c}, s_{c d}$, $s_{e a}, s_{a c}, s_{a d}, s_{b d}, s_{b e}, s_{c e}$. These poles reflect the chosen legs for the tree amplitude in the factorisation. In calculating the residues of this chnnel, we may pick any orientation for the remaining three legs on the right hand side of the factorisation since every configuration is accounted for in the full colour decomposition. This

\footnotetext{
${ }^{1}$ For example, this is the excited tree:two-loop factorisation in the $s_{a b}$ channel.
} 
method correctly reconstructs the factorising contribution for the leading piece 1 ,

$$
\begin{aligned}
R_{5: 1}^{(0-2)}(a, b, c, d, e) & =A_{3}^{(0)}\left(a^{+}, b^{+},-K^{-}\right) \frac{-1}{s_{a b}} R_{4: 1}^{(2)}\left(K^{+}, c^{+}, d^{+}, e^{+}\right) \\
& +A_{3}^{(0)}\left(b^{+}, c^{+},-K^{-}\right) \frac{-1}{s_{b c}} R_{4: 1}^{(2)}\left(K^{+}, d^{+}, e^{+}, a^{+}\right) \\
& +A_{3}^{(0)}\left(c^{+}, d^{+},-K^{-}\right) \frac{-1}{s_{c d}} R_{4: 1}^{(2)}\left(K^{+}, e^{+}, a^{+}, b^{+}\right) \\
& +A_{3}^{(0)}\left(e^{+}, a^{+},-K^{-}\right) \frac{-1}{s_{e a}} R_{4: 1}^{(2)}\left(K^{+}, b^{+}, c^{+}, d^{+}\right)
\end{aligned}
$$

The rational term, $R_{5: 2}^{(0-2)}(a ; b, c, d, e)$, has contributions from factorisations

$$
\begin{aligned}
R_{5: 2}^{(0-2)}(a ; b, c, d, e) & =A_{3}^{(0)}\left(b^{+}, c^{+},-K^{-}\right) \frac{-1}{s_{b c}} R_{4: 2}^{(2)}\left(K^{+}, a^{+}, d^{+}, e^{+}\right) \\
& +A_{3}^{(0)}\left(c^{+}, d^{+},-K^{-}\right) \frac{-1}{s_{c d}} R_{4: 2}^{(2)}\left(K^{+}, a^{+}, b^{+}, e^{+}\right) \\
& +A_{3}^{(0)}\left(e^{+}, b^{+},-K^{-}\right) \frac{-1}{s_{e b}} R_{4: 2}^{(2)}\left(K^{+}, a^{+}, c^{+}, d^{+}\right)
\end{aligned}
$$

In general, there is another factorisation that is picked up from the colour algebra, the factorisation on the pole $s_{d e}$, however this is not excited by the shift.

$R_{5: 3}^{(2)}(a, b ; c, d, e)$, has factorisations,

$$
\begin{aligned}
R_{5: 3}^{(0-2)}(a, b ; c, d, e) & =A_{3}^{(0)}\left(c^{+}, d^{+},-K^{-}\right) \frac{-1}{s_{c d}} R_{4: 3}^{(2)}\left(K^{+}, a^{+}, b^{+}, e^{+}\right) \\
& +A_{3}^{(0)}\left(e^{+}, c^{+},-K^{-}\right) \frac{-1}{s_{e c}} R_{4: 3}^{(2)}\left(K^{+}, a^{+}, b^{+}, d^{+}\right)
\end{aligned}
$$

again here the factorisation in the $s_{d e}$ channel is present but not excited.

$R_{5: 1,1}^{(2)}(a ; b ; c, d, e)$, has factorisations,

$$
\begin{aligned}
R_{5: 1,1}^{(0-2)}(a ; b ; c, d, e) & =A_{3}^{(0)}\left(c^{+}, d^{+},-K^{-}\right) \frac{-1}{s_{c d}} R_{4: 1,1}^{(2)}\left(K^{+} ; a^{+} ; b^{+}, e^{+}\right) \\
& +A_{3}^{(0)}\left(e^{+}, c^{+},-K^{-}\right) \frac{-1}{s_{e c}} R_{4: 1,1}^{(2)}\left(K^{+} ; a^{+} ; b^{+}, d^{+}\right)
\end{aligned}
$$

once more, the factorisation in the $s_{d e}$ channel is present but not excited.

If we were to include the $s_{d e}$ channel, it is clear that the poles in the subleading

\footnotetext{
${ }^{1}$ Here, the legs $p_{a}, p_{b}$ and $p_{c}$ are shifted in the recursion.
} 


\section{COLOUR DRESSED UNITARITY AND RECURSION FOR YANG-MILLS TWO-LOOP ALL-PLUS AMPLITUDES}

rational terms are poles in all adjacent legs in the trace structure with the larger amount of elements. At the $R_{5: 2}^{(2)}$ level this is $\operatorname{Tr}(b c d e)$. The only term that has no contribution from this factorisation channel is the sub-subleading single trace rational piece, $R_{5: 1 B}^{(2)}$. This is an indication that this partial amplitude does not behave in a conventional fashion, we will see another fact about this amplitude in the one-loop:one-loop factorisations.

Given that we have accounted for the simple pole contributions from the tree to two-loop factorisations, we move on to the one-loop to one-loop factorisations.

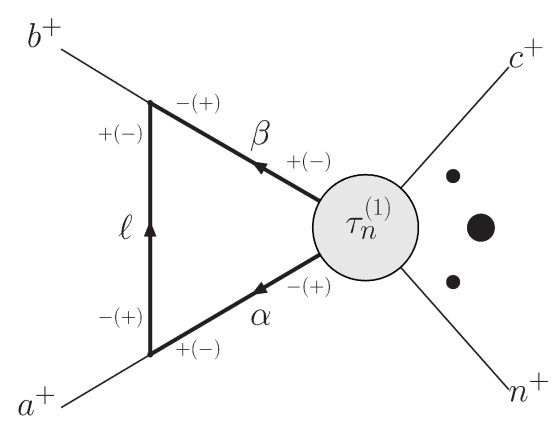

Figure 4.5: Diagram containing the leading and sub-leading poles as $s_{a b} \rightarrow 0$. The axial gauge construction permits the off-shell continuation of the internal legs.

The one-loop to one-loop factorisations involve double poles which are readily available from factorisation theorems, however we need to determine the subleading poles in their Laurent expansion. By considering the familiar diagram fig. 4.5 using an axial gauge formalism, we can determine the full pole structure of the rational piece, including the non-factorising simple poles using augmented recursion. In the full colour context, the double pole structure is determined by calculating the full colour doubly off-shell current $\tau_{n}^{(1), c}\left(\alpha^{-}, \beta^{+}, c^{+}, \ldots, n^{+}\right)$where the two off-shell legs are,

$$
\alpha=\alpha(\ell)=\ell+a \text { and } \beta=\beta(l)=b-\ell \text {. }
$$

The sum of these legs, $P_{\alpha \beta}=\alpha+\beta=a+b=P_{a b}$, is independent of the loop momentum, $\ell$. 
The colour dressed principal helicity assignment in fig. 4.5, gives

$$
\int d \Lambda^{c}\left(\alpha^{+}, a^{+}, b^{+}, \beta^{-}\right) \tau_{n}^{(1), c}\left(\alpha^{-}, \beta^{+}, c^{+}, \ldots, n^{+}\right),
$$

where

$$
\int d \Lambda^{c}\left(\alpha^{+}, a^{+}, b^{+}, \beta^{-}\right) \equiv \frac{i}{c_{\Gamma}(2 \pi)^{D}} \int \frac{d^{D} \ell}{\ell^{2} \alpha^{2} \beta^{2}} \mathcal{A}_{3}^{(0)}(\alpha, a, \ell) \mathcal{A}_{3}^{(0)}(\ell, b, \beta) .
$$

The three-point vertices, $\mathcal{A}_{3}^{(0)}$ carry the colour factors associated with three-point trees, i.e.

$$
\mathcal{A}_{3}^{(0)}(a, b, c)=[\operatorname{Tr}(a b c)-\operatorname{Tr}(c b a)] A_{3}^{(0)}(a, b, c) .
$$

The full colour method differs from the methods applied at leading order in the same way that unitarity did, we simply write the full colour contribution of the component amplitudes.

As we are only interested in the residue on the $s_{a b} \rightarrow 0$ pole, we do not need the exact current. It is sufficient that the approximate current satisfies two conditions [84, 86]:

(C1) The current contains the leading singularity as $s_{\alpha \beta} \rightarrow 0$ with $\alpha^{2}, \beta^{2} \neq 0$,

(C2) The current is the one-loop, single-minus amplitude in the on-shell limit $\alpha^{2}, \beta^{2} \rightarrow 0, s_{\alpha \beta} \neq 0$.

This process is detailed in subsection 2.10.1 and [88].

We now apply the method to the full colour amplitude. The $U\left(N_{c}\right)$ colour decomposition of the integral measure, $d \Lambda^{c}$ contains a common kinematic factor so we have the colour decompositions

$$
\tau_{n}^{(1), c}=\sum_{\lambda} C_{\lambda} \tau_{n: \lambda}^{(1)} \text { and } \int d \Lambda^{c}=C_{\Lambda} \int d \Lambda_{0}
$$

where

$$
\int d \Lambda_{0}\left(\alpha^{+}, a^{+}, b^{+}, \beta^{-}\right)=\frac{i}{(2 \pi)^{D}} \int \frac{d^{D} \ell}{\ell^{2} \alpha^{2} \beta^{2}} \frac{[a|\ell| q\rangle[b|\ell| q\rangle}{\langle a q\rangle\langle b q\rangle} \frac{\langle\beta q\rangle^{2}}{\langle\alpha q\rangle^{2}} .
$$




\section{COLOUR DRESSED UNITARITY AND RECURSION FOR YANG-MILLS TWO-LOOP ALL-PLUS AMPLITUDES}

Hence the full colour contribution is

$$
\sum_{\lambda} C_{\Lambda} C_{\lambda} \int d \Lambda_{0}\left(\alpha^{+}, a^{+}, b^{+}, \beta^{-}\right) \tau_{n: \lambda}^{(1)}\left(\alpha^{-}, \beta^{+}, c^{+}, \cdots n^{+}\right) .
$$

The current is derived from the one-loop five-point single-minus amplitude and consequently has the same colour decomposition. The fact that we take two legs off-shell here only affects the kinematics - colour is unaffected and has the same colour algebra.

This decomposition is schematic, it illustrates the point that traces sourced from the two tree amplitudes combine with all the trace structures from the oneloop decomposition. Performing the colour algebra while identifying the positions of the off-shell legs in the functional form of the current with respect to the on-shell legs, allows us to understand what types of currents will arise in the subleading rational pieces. Since this is a one-loop current, we may also use the useful property that the various $\tau_{n: \lambda}^{(1)}$ can be expressed as sums of the leading amplitudes $\tau_{n: 1}^{(1)}$ via a series of $U(1)$-decoupling identities. This is useful in this case because the leading in colour current was calculated in [86] and rederived for a general $\lambda_{q}$ for this calculation (presented in subsection 2.10.1).

We now focus on the five-point case, where we show that the only two objects we need to calculate for the one-loop:one-loop factorisations are two distinct forms of the leading current,

$$
\tau_{5: 1}^{(1)}\left(\alpha^{-}, \beta^{+}, c^{+}, d^{+}, e^{+}\right) \text {and } \tau_{5: 1}^{(1)}\left(\alpha^{-}, c^{+}, \beta^{+}, d^{+}, e^{+}\right)
$$

which we call the 'adjacent' and 'non-adjacent' leading currents respectively.

Performing the colour algebra with the functional form of the current uncovers 


\subsection{Recursion}

that the $S U\left(N_{c}\right)$ rational terms $R_{5: 3}^{(2)}$ and $R_{5: 1 B}^{(2)}$ get contributions of the form,

$$
\begin{aligned}
& R_{5: 3}^{(1-1)}(a, b ; c, d, e) \\
& =\operatorname{Res}\left[\left\{\int d \Lambda _ { 0 } ( \alpha ^ { + } , \hat { b } ^ { + } , \hat { c } ^ { + } , \beta ^ { - } ) \left(\tau_{5: 1}^{(1)}\left(d^{+}, e^{+}, \beta^{+}, \hat{a}^{+}, \alpha^{-}\right)+\tau_{5: 1}^{(1)}\left(e^{+}, d^{+}, \beta^{+}, \hat{a}^{+}, \alpha^{-}\right)\right.\right.\right. \\
& \left.+\tau_{5: 3}^{(1)}\left(d^{+}, e^{+} ; \beta^{+}, \hat{a}^{+}, \alpha^{-}\right)\right) \\
& +\int d \Lambda_{0}\left(\alpha^{+}, \hat{a}^{+}, \hat{b}^{+}, \beta^{-}\right)\left(\tau_{5: 1}^{(1)}\left(d^{+}, e^{+}, \beta^{+}, \hat{c}^{+}, \alpha^{-}\right)+\tau_{5: 1}^{(1)}\left(e^{+}, d^{+}, \beta^{+}, \hat{c}^{+}, \alpha^{-}\right)\right. \\
& \left.+\tau_{5: 3}^{(1)}\left(d^{+}, e^{+} ; \beta^{+}, \hat{c}^{+}, \alpha^{-}\right)\right) \\
& +\int d \Lambda_{0}\left(\alpha^{+}, \hat{a}^{+}, \hat{c}^{+}, \beta^{-}\right)\left(\tau_{5: 1}^{(1)}\left(\hat{b}^{+}, \beta^{+}, d^{+}, e^{+}, \alpha^{-}\right)+\tau_{5: 1}^{(1)}\left(\hat{b}^{+}, \beta^{+}, e^{+}, d^{+}, \alpha^{-}\right)\right. \\
& \left.-\tau_{5: 3}^{(1)}\left(d^{+}, e^{+} ; \beta^{+}, \hat{b}^{+}, \alpha^{-}\right)\right) \\
& -\int d \Lambda_{0}\left(\alpha^{+}, d^{+}, \hat{a}^{+}, \beta^{-}\right)\left(\tau_{5: 1}^{(1)}\left(d^{+}, \beta^{+}, \hat{b}^{+}, \hat{c}^{+}, \alpha^{-}\right)+\tau_{5: 1}^{(1)}\left(\hat{b}^{+}, \hat{c}^{+}, \beta^{+}, d^{+}, \alpha^{-}\right)\right) \\
& -\int d \Lambda_{0}\left(\alpha^{+}, d^{+}, \hat{b}^{+}, \beta^{-}\right)\left(\tau_{5: 1}^{(1)}\left(d^{+}, \beta^{+}, \hat{c}^{+}, \hat{a}^{+}, \alpha^{-}\right)+\tau_{5: 1}^{(1)}\left(\hat{c}^{+}, \hat{a}^{+}, \beta^{+}, d^{+}, \alpha^{-}\right)\right) \\
& -\int d \Lambda_{0}\left(\alpha^{+}, e^{+}, \hat{c}^{+}, \beta^{-}\right)\left(\tau_{5: 1}^{(1)}\left(d^{+}, \beta^{+}, \hat{a}^{+}, \hat{b}^{+}, \alpha^{-}\right)+\tau_{5: 1}^{(1)}\left(\hat{a}^{+}, \hat{b}^{+}, \beta^{+}, d^{+}, \alpha^{-}\right)\right) \\
& -\int d \Lambda_{0}\left(\alpha^{+}, d^{+}, \hat{a}^{+}, \beta^{-}\right)\left(\tau_{5: 1}^{(1)}\left(e^{+}, \beta^{+}, \hat{b}^{+}, \hat{c}^{+}, \alpha^{-}\right)+\tau_{5: 1}^{(1)}\left(\hat{b}^{+}, \hat{c}^{+}, \beta^{+}, e^{+}, \alpha^{-}\right)\right) \\
& -\int d \Lambda_{0}\left(\alpha^{+}, d^{+}, \hat{b}^{+}, \beta^{-}\right)\left(\tau_{5: 1}^{(1)}\left(e^{+}, \beta^{+}, \hat{c}^{+}, \hat{a}^{+}, \alpha^{-}\right)+\tau_{5: 1}^{(1)}\left(\hat{c}^{+}, \hat{a}^{+}, \beta^{+}, e^{+}, \alpha^{-}\right)\right) \\
& \left.-\int d \Lambda_{0}\left(\alpha^{+}, d^{+}, \hat{c}^{+}, \beta^{-}\right)\left(\tau_{5: 1}^{(1)}\left(\hat{a}^{+}, \hat{b}^{+}, \beta^{+}, e^{+}, \alpha^{-}\right)+\tau_{5: 1}^{(1)}\left(e^{+}, \beta^{+}, \hat{a}^{+}, \hat{b}^{+}, \alpha^{-}\right)\right)\right\} \\
& +\{\text {Other Helicity Configuration }\}] \text {, }
\end{aligned}
$$

and 


\section{COLOUR DRESSED UNITARITY AND RECURSION FOR YANG-MILLS TWO-LOOP ALL-PLUS AMPLITUDES}

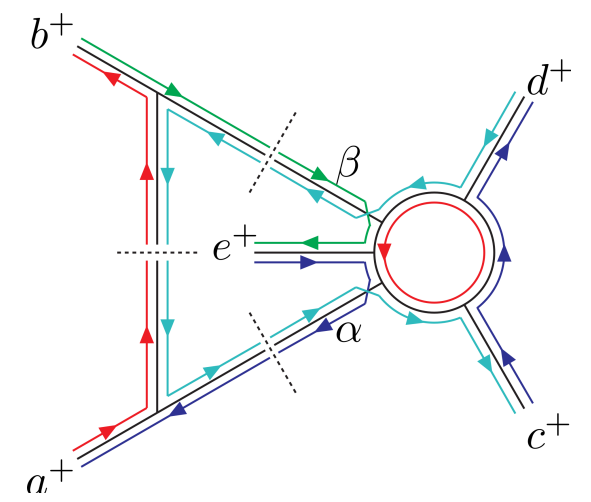

Figure 4.6: The rational piece, $R_{5: 3}^{(2)}$, multiplying the trace structure $N_{c} \operatorname{Tr}[a b] \operatorname{Tr}[c d e]$, is constructed from currents of the form $\tau_{5: 1}^{(1)}\left(\alpha^{ \pm}, c^{+} ; \beta^{\mp}, d^{+}, e^{+}\right)$.

$$
\begin{aligned}
& R_{5: 1 B}^{(1-1)}(a, b, c, d, e) \\
& =\operatorname{Res}\left[\left\{\int d \Lambda_{0}\left(\alpha^{+}, \hat{b}^{+}, \hat{c}^{+}, \beta^{-}\right)\left(\tau_{5: 3}^{(1)}\left(\alpha^{-}, \hat{a}^{+} ; \beta^{+}, d^{+}, e^{+}\right)+\tau_{5: 3}^{(1)}\left(\beta^{+}, d^{+} ; \alpha^{-}, e^{+}, \hat{a}^{+}\right)\right)\right.\right. \\
& +\int d \Lambda_{0}\left(\alpha^{+}, \hat{a}^{+}, \hat{b}^{+}, \beta^{-}\right)\left(\tau_{5: 3}^{(1)}\left(\alpha^{-}, e^{+} ; \beta^{+}, \hat{c}^{+}, d^{+}\right)+\tau_{5: 3}^{(1)}\left(\beta^{+}, \hat{c}^{+} ; \alpha^{-}, d^{+}, e^{+}\right)\right) \\
& -\int d \Lambda_{0}\left(\alpha^{+}, \hat{a}^{+}, \hat{c}^{+}, \beta^{-}\right)\left(\tau_{5: 3}^{(1)}\left(\alpha^{-}, \hat{b}^{+} ; \beta^{+}, d^{+}, e^{+}\right)+\tau_{5: 3}^{(1)}\left(\beta^{+}, \hat{b}^{+} ; \alpha^{-}, d^{+}, e^{+}\right)\right) \\
& +\int d \Lambda_{0}\left(\alpha^{+}, e^{+}, \hat{a}^{+}, \beta^{-}\right)\left(\tau_{5: 3}^{(1)}\left(\alpha^{-}, d^{+} ; \beta^{+}, \hat{b}^{+}, \hat{c}^{+}\right)+\tau_{5: 3}^{(1)}\left(\beta^{+}, \hat{b}^{+} ; \alpha^{-}, \hat{c}^{+}, d^{+}\right)\right) \\
& +\int d \Lambda_{0}\left(\alpha^{+}, e^{+}, \hat{b}^{+}, \beta^{-}\right)\left(\tau_{5: 3}^{(1)}\left(\alpha^{-}, \hat{a}^{+} ; \beta^{+}, d^{+}, \hat{c}^{+}\right)+\tau_{5: 3}^{(1)}\left(\beta^{+}, \hat{a}^{+} ; \alpha^{-}, d^{+}, \hat{c}^{+}\right)\right) \\
& -\int d \Lambda_{0}\left(\alpha^{+}, e^{+}, \hat{c}^{+}, \beta^{-}\right)\left(\tau_{5: 3}^{(1)}\left(\alpha^{-}, d^{+} ; \beta^{+}, \hat{a}^{+}, \hat{b}^{+}\right)+\tau_{5: 3}^{(1)}\left(\beta^{+}, d^{+} ; \alpha^{-}, \hat{a}^{+}, \hat{b}^{+}\right)\right) \\
& -\int d \Lambda_{0}\left(\alpha^{+}, d^{+}, \hat{a}^{+}, \beta^{-}\right)\left(\tau_{5: 3}^{(1)}\left(\alpha^{-}, e^{+} ; \beta^{+}, \hat{b}^{+}, \hat{c}^{+}\right)+\tau_{5: 3}^{(1)}\left(\beta^{+}, e^{+} ; \alpha^{-}, \hat{b}^{+}, \hat{c}^{+}\right)\right) \\
& -\int d \Lambda_{0}\left(\alpha^{+}, d^{+}, \hat{b}^{+}, \beta^{-}\right)\left(\tau_{5: 3}^{(1)}\left(\alpha^{-}, \hat{c}^{+} ; \beta^{+}, e^{+}, \hat{a}^{+}\right)+\tau_{5: 3}^{(1)}\left(\beta^{+}, \hat{c}^{+} ; \alpha^{-}, e^{+}, \hat{a}^{+}\right)\right) \\
& \left.+\int d \Lambda_{0}\left(\alpha^{+}, d^{+}, \hat{c}^{+}, \beta^{-}\right)\left(\tau_{5: 3}^{(1)}\left(\alpha^{-}, \hat{b}^{+} ; \beta^{+}, e^{+}, \hat{a}^{+}\right)+\tau_{5: 3}^{(1)}\left(\beta^{+}, e^{+} ; \alpha^{-}, \hat{a}^{+}, \hat{b}^{+}\right)\right)\right\} \\
& +\{\mathrm{Other} \text { Helicity } \text { Configuration }\}] .
\end{aligned}
$$

In general, we see the appearance of non-adjacent $\tau_{5: 1}$ currents and $\tau_{5: 3}$ currents for which the off-shell legs appear in both different sets and in the same set, 


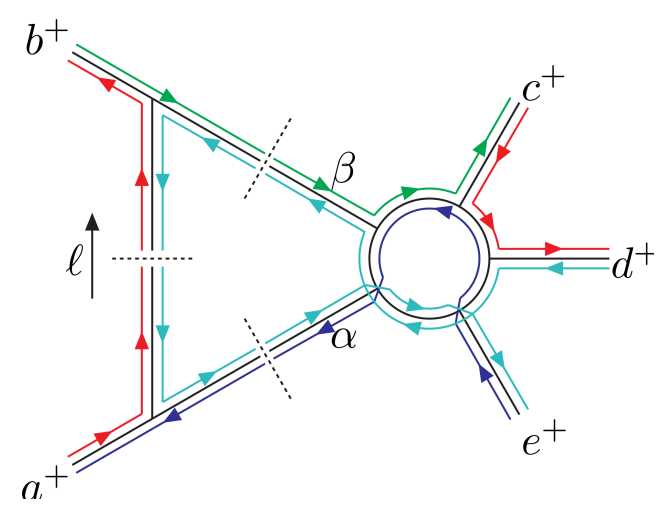

Figure 4.7: The maximally non-factorising rational piece, $R_{5: 1 B}^{(2)}$, multiplying the trace structure $\operatorname{Tr}[a b c d e]$, is constructed from currents of the form $\tau_{5: 3}^{(1)}\left(\alpha^{ \pm}, e^{+} ; \beta^{\mp}, c^{+}, d^{+}\right)$.

i.e. $\tau_{5: 3}\left(\alpha^{-}, b^{+} ; \beta^{+}, d^{+}, e^{+}\right)$and $\tau_{5: 3}\left(d^{+}, e^{+} ; \alpha^{-}, \beta^{+}, a^{+}\right)$. We may analyse the decoupling identities of both types of subleading currents to confirm that only the adjacent and non-adjacent $\tau_{5: 1}$ currents are required. The decoupling identity is given as

$$
A_{5: 3}^{(1)}(1,2 ; 3,4,5)=\sum_{\sigma \in C O P_{4}^{(123)}} A_{5: 1}^{(1)}(\sigma(1), \sigma(2), \sigma(3), \sigma(4), 5)
$$

which, for the currents given above translates to,

$$
\begin{aligned}
A_{5: 3}^{(1)}(\alpha, b ; \beta, d, e) & =A_{5: 1}^{(1)}(\alpha, b, \beta, d, e)+A_{5: 1}^{(1)}(\alpha, b, d, e, \beta)+A_{5: 1}^{(1)}(\alpha, b, e, \beta, d)+A_{5: 1}^{(1)}(\alpha, \beta, b, d, e) \\
& +A_{5: 1}^{(1)}(\alpha, d, b, e, \beta)+A_{5: 1}^{(1)}(\alpha, e, b, \beta, d)+A_{5: 1}^{(1)}(\alpha, \beta, d, b, e)+A_{5: 1}^{(1)}(\alpha, d, e, b, \beta) \\
& +A_{5: 1}^{(1)}(\alpha, e, \beta, b, d)+A_{5: 1}^{(1)}(\alpha, \beta, d, e, b)+A_{5: 1}^{(1)}(\alpha, d, e, \beta, b)+A_{5: 1}^{(1)}(\alpha, e, \beta, d, b),
\end{aligned}
$$

and

$$
\begin{aligned}
A_{5: 3}^{(1)}(a, b ; \alpha, \beta, d) & =A_{5: 1}^{(1)}(a, b, \alpha, \beta, d)+A_{5: 1}^{(1)}(a, b, \beta, d, \alpha)+A_{5: 1}^{(1)}(a, b, d, \alpha, \beta)+A_{5: 1}^{(1)}(a, \alpha, b, \beta, d) \\
& +A_{5: 1}^{(1)}(a, \beta, b, d, \alpha)+A_{5: 1}^{(1)}(a, d, b, \alpha, \beta)+A_{5: 1}^{(1)}(a, \alpha, \beta, b, d)+A_{5: 1}^{(1)}(a, \beta, d, b, \alpha) \\
& +A_{5: 1}^{(1)}(a, d, \alpha, b, \beta)+A_{5: 1}^{(1)}(a, \alpha, \beta, d, b)+A_{5: 1}^{(1)}(a, \beta, d, \alpha, b)+A_{5: 1}^{(1)}(a, d, \alpha, \beta, b) .
\end{aligned}
$$




\section{COLOUR DRESSED UNITARITY AND RECURSION FOR YANG-MILLS TWO-LOOP ALL-PLUS AMPLITUDES}

These expressions confirm that both the leading adjacent and non-adjacent currents appear at subleading in colour partial amplitudes. This is supported by the observation that the only possible doubly off-shell currents that can exist at five point are just the leading adjacent and non-adjacent currents. Despite the addition of an extra current to compute, this is promising since at six-point there is just one additional current on top of these, that is required.

$\tau_{5: 1}^{(1)}\left(\alpha^{-}, \beta^{+}, c^{+}, d^{+}, e^{+}\right)$has been calculated previously for a specific choice of the axial gauge spinor $\lambda_{q}=\lambda_{d}$ [86. Since in the full colour result we require currents for which all the legs have been permuted, it is necessary to derive this current for arbitrary $\lambda_{q}$. This general formulation will avoid any chance of exciting spurious poles in the rational expression. The non-adjacent case has not previously been considered. The derivation of the adjacent current is given in subsection 2.10.1. A major difference in the derivation of the current in this paper to the current derived in [86] is that the term $\mathcal{F}_{d p}^{c d e}$ describing the leading factorisation of the current had to be exposed explicitly since $\lambda_{q}$ was kept general. This point is touched on in more depth in subsection 2.10.1.

This current is given by,

$$
\begin{gathered}
\tau_{5: 1}^{(1)}\left(\alpha^{-}, \beta^{+}, c^{+}, d^{+}, e^{+}\right)=\mathcal{F}_{d p}^{c d e}\left[1+s_{\alpha \beta}\left(\frac{[q e]}{[c e]\left[q\left|P_{\alpha \beta}\right| c\right\rangle}+\frac{[c|q| d\rangle}{\left[q\left|P_{\alpha \beta}\right| q\right\rangle[c|e| d\rangle}+\frac{[e|q| d\rangle}{\left[q\left|P_{\alpha \beta}\right| q\right\rangle[e|c| d\rangle}\right)\right] \\
+\frac{i}{3\langle c d\rangle^{2}} \frac{\langle\alpha q\rangle^{2}}{\langle\beta q\rangle^{2}}\left[\frac{\langle\alpha c\rangle[c|\beta| d\rangle}{\langle d e\rangle\langle e \alpha\rangle}+\frac{\langle c e\rangle[d e]}{\langle d e\rangle^{2}}\left(\frac{\left[q\left|P_{\alpha \beta}\right| d\right\rangle^{3}}{\left[q\left|P_{\alpha \beta}\right| q\right\rangle^{3}} \frac{\langle q c\rangle\langle q \alpha\rangle[q|\alpha| q\rangle}{\langle\alpha c\rangle\left[q\left|P_{\alpha \beta}\right| c\right\rangle}-3 \frac{\langle q d\rangle\left[q\left|P_{\alpha \beta}\right| d\right\rangle^{2}[q|\beta| q\rangle}{\left[q\left|P_{\alpha \beta}\right| q\right\rangle^{2}\left[q\left|P_{\alpha \beta}\right| c\right\rangle}\right)\right] \\
+\mathcal{F}_{s b}^{c d e}+\frac{i}{3\langle c d\rangle^{2}}\left(-\frac{[\beta e]^{2}[q e]}{[e \alpha][\alpha q]}+[e|q| \alpha\rangle \frac{\left([e \beta][\beta q]\left[q\left|P_{\alpha \beta}\right| q\right\rangle-[\beta q]^{2}\left[e\left|P_{\alpha \beta}\right| q\right\rangle\right)}{[\alpha q]\left[q\left|P_{\alpha \beta}\right| q\right\rangle^{2}}\right) \\
+\mathcal{O}(\langle\alpha \beta\rangle)+\mathcal{O}\left(\alpha^{2}\right)+\mathcal{O}\left(\beta^{2}\right),
\end{gathered}
$$

where,

$$
\mathcal{F}_{d p}^{c d e}=\frac{i}{3} \frac{\langle\alpha q\rangle^{2}}{\langle\beta q\rangle^{2}} \frac{\langle q|\alpha \beta| q\rangle}{s_{\alpha \beta}} \frac{\langle e c\rangle[c e]^{3}}{\langle c d\rangle\langle d e\rangle\left[e\left|P_{\alpha \beta}\right| q\right\rangle\left[c\left|P_{\alpha \beta}\right| q\right\rangle}
$$


and

$$
\mathcal{F}_{s b}^{c d e}=-\frac{i}{3} \frac{\left[e\left|P_{\alpha \beta}\right| \alpha\right\rangle[\beta q]^{2}}{[\alpha q]\left[q\left|P_{\alpha \beta}\right| q\right\rangle} \frac{1}{s_{\alpha \beta}} \frac{\left[e\left|P_{\alpha \beta}\right| q\right\rangle}{\langle c d\rangle^{2}} .
$$

Setting $\lambda_{q}=\lambda_{d}$ in (4.82) reproduces the current presented in [86].

The non-adjacent leading current is derived from the five-point single minus with the off-shell legs non-adjacent. The current has no pole in $\langle\alpha \beta\rangle$ and is therefore much simpler. Although this current will not lead to a double pole in $\langle\alpha \beta\rangle$, it has a non-planar factorisation. Since augmented recursion automatically encodes this property, we can safely capture the desired behaviour without having to worry about the intricacies of a non-planar factorisation.

The same conditions apply to this current and so we start with the amplitude,

$$
A_{5: 1}^{(1)}\left(\alpha^{-}, c^{+}, \beta^{+}, d^{+}, e^{+}\right)=\frac{i}{3} \frac{1}{\langle\beta d\rangle^{2}}\left(\frac{\langle c d\rangle\langle\alpha \beta\rangle^{3}[c \beta]}{\langle c \beta\rangle^{2}\langle d e\rangle\langle\alpha e\rangle}+\frac{\langle\alpha d\rangle^{3}\langle\beta e\rangle[d e]}{\langle c \beta\rangle\langle d e\rangle^{2}\langle\alpha c\rangle}-\frac{[c e]^{3}}{[e \alpha][\alpha c]}\right) .
$$

The first term is $\mathcal{O}\left(\langle\alpha \beta\rangle^{3}\right)$ which means we can discard it. The same leading approximations can be used as in the previous current to derive

$$
\tau_{5: 1}^{(1)}\left(\alpha^{-}, c^{+}, \beta^{+}, d^{+}, e^{+}\right)=\frac{i}{3} \frac{\langle\alpha q\rangle^{2}}{\langle\beta q\rangle^{2}}\left(\frac{\langle\alpha e\rangle[e c]}{\langle c \alpha\rangle\langle d e\rangle^{2}}-\frac{[e c]^{3}}{[e|\alpha| d\rangle[c|\alpha| d\rangle}\right)+\mathcal{O}(\langle\alpha \beta\rangle) .
$$

Integrating the current into the triangle diagram responsible for capturing the rational terms, the non-adjacent case integrates to the simple form,

$$
\int \frac{d^{D} \ell}{\ell^{2} \alpha^{2} \beta^{2}} \frac{i}{3} \frac{[a|\ell| q\rangle[b|\ell| q\rangle}{\langle a q\rangle\langle b q\rangle} \frac{\langle a e\rangle[e c]}{\langle c a\rangle\langle d e\rangle^{2}}=\frac{i}{6} \frac{[e c]\langle a e\rangle[a b]}{\langle d e\rangle^{2}\langle c a\rangle\langle a b\rangle} .
$$

where the second term in eq. (4.86) has been dropped since it is a quadratic pentagon and does not contain any rational terms. The integrated adjacent case is a generalisation of the previous result [86]. Summing over all the channels excited by the Risager shift, including both the tree to two-loop and one-loop to one-loop factorisations, we recover the full two-loop colour decomposition. We 


\section{COLOUR DRESSED UNITARITY AND RECURSION FOR YANG-MILLS TWO-LOOP ALL-PLUS AMPLITUDES}

present compact forms of the $S U\left(N_{c}\right)$ rational pieces below, including the first compact form for the rational piece of $R_{5: 1 B}^{(2)}$, obtained via a direct computation. These $\lambda_{q}$-independent functions can be reconstructed from the analytic forms computed using the two independent currents. However, in this computation we confirmed our result for $R_{5: 3}^{(2)}$ against the explicitly $\lambda_{q}$-independent result calculated by [110] finding complete agreement. Our $R_{5: 1 B}^{(2)}$ satisfies the constraint (4.24) and consequently we utilised this along with the decoupling identities to generate explicitly $\lambda_{q}$-independent results for the remaining $U\left(N_{c}\right)$ and $S U\left(N_{c}\right)$ amplitudes.

$R_{5: 1}^{(2)}\left(a^{+}, b^{+}, c^{+}, d^{+}, e^{+}\right)=\frac{i}{9} \frac{1}{\langle a b\rangle\langle b c\rangle\langle c d\rangle\langle d e\rangle\langle e a\rangle} \sum_{S_{5: 1}}\left(\frac{\operatorname{tr}_{+}^{2}[d e a b]}{s_{d e} s_{a b}}+5 s_{a b} s_{b c}+s_{a b} s_{c d}\right)$

$R_{5: 3}^{(2)}\left(a^{+}, b^{+} ; c^{+}, d^{+}, e^{+}\right)=\frac{2 i}{3} \frac{1}{\langle a b\rangle\langle b a\rangle\langle c d\rangle\langle d e\rangle\langle e c\rangle} \sum_{S_{5: 3}}\left(\frac{\operatorname{tr}_{-}[a c d e] \operatorname{tr}_{-}[e c b a]}{s_{a e} s_{c d}}+\frac{3}{2} s_{a b}^{2}\right)$,

and

$$
\begin{array}{r}
R_{5: 1 B}^{(2)}\left(a^{+}, b^{+}, c^{+}, d^{+}, e^{+}\right)=2 i \varepsilon(a, b, c, d)\left(\mathrm{C}_{\mathrm{PT}}(a, b, e, c, d)+\mathrm{C}_{\mathrm{PT}}(a, d, b, c, e)+\right. \\
\mathrm{C}_{\mathrm{PT}}(a, b, d, e, c)+\mathrm{C}_{\mathrm{PT}}(a, c, d, b, e)+ \\
\left.\mathrm{C}_{\mathrm{PT}}(a, d, e, b, c)\right),
\end{array}
$$

where

$$
\mathrm{C}_{\mathrm{PT}}(a, b, c, d, e)=\frac{1}{\langle a b\rangle\langle b c\rangle\langle c d\rangle\langle d e\rangle\langle e a\rangle} .
$$

These expressions are valid for both $U\left(N_{c}\right)$ and $S U\left(N_{c}\right)$ gauge groups and are remarkably compact.

We note that there are double poles at leading and sub-leading in colour, but not at sub-sub-leading. As $R_{4: 1 B}^{(2)}$ vanishes [92] the poles in $R_{5: 1 B}^{(2)}$ do not correspond to tree to two-loop factorisations, instead they arise from contributions of the type shown in fig. 4.5 however the lack of double poles indicates that the terms 
containing double poles cancel. Together these two facts show that $R_{5: 1 B}^{(2)}$ is a purely non-factorising object.

\subsection{Conclusion}

In this chapter we have shown how to extend the methods of four-dimensional unitarity and recursion to the computation of full colour amplitudes and applied such methods to the computation of the full colour five-point all-plus amplitude. Using both $U\left(N_{c}\right)$ Yang-Mills and fully colour dressing amplitudes, we have shown that the simplifications afforded to us in the leading in colour case extends to full colour amplitudes as well. In doing this we have developed a direct method of calculating every partial amplitude that a full colour two-loop all-plus amplitude is comprised of.

Here we calculated the polylogarithmic parts and rational parts of all $U\left(N_{c}\right)$ partial amplitudes present in this full colour amplitude, finding complete agreement with [110] whilst also using decoupling identities and group theory relations to verify the results.

Colour dressed unitarity and recursion holds true to the recursive principles of building unknown rational parts of amplitudes from simpler loop amplitudes including those with lower multiplicity. In this vein the method holds great promise for obtaining all- $n$ expressions for two-loop subleading in colour amplitudes. In fact, we have since seen the calculation of $R_{n: 1 B}^{(2)}$ extended from the simple form found here to an alln expression [90] and the two-loop six-point all-plus amplitude has since been computed [91].

Having discussed pure Yang-Mills calculations, we now proceed to the application of the methods utilised here in a gravitational context. 
4. COLOUR DRESSED UNITARITY AND RECURSION FOR YANG-MILLS TWO-LOOP ALL-PLUS AMPLITUDES 


\section{Chapter 5}

\section{Diagrammar in an Extended Theory of Gravity}

\subsection{Introduction}

There's a lot of information that can be gleamed from the previously investigated work carried out in Yang-Mills. Yang-Mills amplitudes can be defined from a quantum field theory by calculating the Feynman diagrams constructed from Feynman rules derived from the aforementioned quantum field theory. We have since demonstrated that for higher multiplicity amplitudes, utilising complex momenta and factorisations in a recursive approach [35, 36] is more suited to tree-level calculations. Although the factorisation properties of tree-level amplitudes and the momentum power counting of complex three-point amplitudes arose from the analysis performed using Feynman rules and Feynman diagrams, we have since diverged from the original approach. That being said, although employing the spinor helicity formalism and complex recursion is a far better method computationally, there is still a link to the field theory approach. This is pertinent when considering the scattering amplitudes that arise in the analysis of theories of linearised gravity.

The expansion of the gravitational field in a flat spacetime generates an infinite series in the graviton $h_{\mu \nu}$. In a flat spacetime we can have non-interacting states that are well defined at infinity and as such hard scattering events can be described by the $S$-matrix. Therefore, the infinite series of graviton interactions corresponds 


\section{DIAGRAMMAR IN AN EXTENDED THEORY OF GRAVITY}

at leading order to a sequence of tree-level $n$-point scattering amplitudes where $n=3,4, \ldots, \infty$.

Since pertubative gravity can be investigated using Feynman diagrams, we have the advantage of already understanding the singularity structure of treelevel amplitudes. This vantage point has allowed the same methods to be applied in the context of perturbative gravity to determine the $S$-matrix elements [44]. This comes with a caveat, although the link to the Feynman diagram approach allows us to utilise factorisation theorems, it naively implies that under a complex shift graviton amplitudes are not well behaved. Calculating gravity amplitudes using on-shell amplitudes and factorisation highlights concealed cancellations between diagrams that ultimately results in shifted quantities that are well behaved, allowing for the recursive construction of higher multiplicity graviton amplitudes from the complex three-point amplitudes [44, 161 163].

This chapter is based on work performed in [112]. In this chapter, we seek to determine the $S$-matrix elements of a theory of massless extended EinsteinHilbert (EH) gravity using similar complex recursive techniques. The extension involves including the term $\alpha R^{3}$, where $\alpha$ is a dimensionful coupling constant. This theory provides the UV counterterms for the two-loop all-plus amplitude in EH gravity [164].

The amplitudes in this theory can be expanded as a power series in $\alpha$,

$$
M_{n}(1, \cdots, n)=\sum_{r=0} \alpha^{r} M_{n}^{(L), \alpha^{r}}(1, \cdots, n)
$$

where $L$ is the number of loops and $M_{n}^{(0), \alpha^{0}}$ is the EH tree-level gravity amplitude. Here, we focus on leading deformation of the theory, i.e. the $r=1$ part of the extended theory.

The theory we are considering would arise using field theory methods from the Lagrangian

$$
L=\int d^{D} x \sqrt{-g}\left(R+C_{\alpha} R_{a b c d} R^{c d e f} R_{e f}^{a b}\right)
$$

where $C_{\alpha}=\alpha / 60$. However, we note that to do so would involve determining increasingly complicated $n$-point vertices as the Lagrangian is expanded in the 
graviton field. As we will see the three-point amplitudes are sufficient to completely determine the tree-level $S$-matrix.

It is not very useful to define a theory by specifying the entire $S$-matrix explicitly but it is an important question whether the $S$-matrix can be defined from a minimal set of data and rules i.e. a "diagrammar" 165. Once a minimal set of amplitudes is specified we aim to construct all other amplitudes by demanding they have the correct symmetries and singularities. Defining the $S$-matrix using its singularities is a long-standing programme [36, 46, 53, 56, 166, 167].

In addition to determining the tree-level $S$-matrix via recursion, we also calculate the soft limits of the graviton amplitudes we calculate. With the soft limit parametrisation, $\lambda_{n}=t \times \lambda_{s}$ and $\bar{\lambda}_{n}=\bar{\lambda}_{s}$, tree level graviton amplitudes are singular, with a Laurent series whose highest order singularity is $t^{-3}$. The coefficients of the terms in the Laurent series are described by soft theorems. Weinberg described the universal leading soft behaviour of a graviton scattering amplitude [117]. The subleading and sub-subleading coefficients are given by [115]. It has been posited that these soft functions are universal 114 116 and in section 5.3 we investigate the claim for this extended theory of gravity.

\subsection{Determining the Tree-Level $S$-Matrix Ele- ments}

The key element in determining the tree-level $S$-matrix is that it is entirely characterised by the three-point vertices if we demand that the amplitudes factorise on simple poles. Since we are working with graviton amplitudes, there is no ordering of the external gravitons as there was with gluons. As such, for any partition of the external legs into two sets, $\left\{p_{L_{1}}, p_{L_{2}} \cdots, p_{L_{l}}\right\}$ and $\left\{p_{R_{1}}, p_{R_{2}} \cdots, p_{R_{m}}\right\}$ with $l+m=n$ and $l, m \geq 2$, if $K=\sum_{j=1}^{l} k_{L_{j}}$, then when $K^{2} \longrightarrow 0$ the amplitude is singular with the simple pole being,

$$
M_{n}^{(0)} \stackrel{K^{2} \rightarrow 0}{\longrightarrow} \sum_{h= \pm} M_{l+1}^{(0)}\left(p_{L_{1}}, \ldots, p_{L_{l}},-K^{h}\right) \frac{-1}{K^{2}} M_{m+1}^{(0)}\left(K^{-h}, p_{R_{1}}, \ldots, p_{R_{m}}\right)
$$

We can excite the pole in $K^{2}$ by utilizing complex momenta and applying 


\section{DIAGRAMMAR IN AN EXTENDED THEORY OF GRAVITY}

methods of complex analysis. There are two shifts which we use to generate the $S$-matrix. First, the BCFW shift [36],

$$
\lambda_{i} \longrightarrow \lambda_{i}+z \lambda_{j}, \bar{\lambda}_{j} \longrightarrow \bar{\lambda}_{j}-z \bar{\lambda}_{i}
$$

For EH gravity, this shift is sufficient to generate the tree level $S$-matrix [163]. Additionally we use the Risager shift [37],

$$
\begin{aligned}
& \lambda_{i} \longrightarrow \lambda_{i}+z[j k] \lambda_{\eta}, \\
& \lambda_{j} \longrightarrow \lambda_{j}+z[k i] \lambda_{\eta}, \\
& \lambda_{k} \longrightarrow \lambda_{k}+z[i j] \lambda_{\eta},
\end{aligned}
$$

where $\lambda_{\eta}$ is a reference spinor. Details involving the use of these shifts can be found in section 2.7. We find that both shifts are required to construct the $S$-matrix for the extended theory. This is due to the fact that there are nonvanishing all-plus graviton amplitudes at $\mathcal{O}(\alpha)$ and from the work performed in Yang-Mills, shown in previous chapters, the BCFW shift does not have the desired asymptotic behaviour as $z \rightarrow \infty$ for the all-plus amplitudes.

In tree-level Yang-Mills amplitudes and EH gravity, the form of the complex three-point amplitudes determines the tree-level $S$-matrix completely. In order to ascertain the $S$-matrix elements at $\mathcal{O}(\alpha)$ we require both the three-point tree-level EH amplitudes and the three-point deformations.

The usual three-point amplitudes of EH gravity are given by:

$$
\begin{aligned}
M_{3}^{(0), \alpha^{0}}\left(a^{-}, b^{-}, c^{+}\right) & =\frac{\langle a b\rangle^{6}}{\langle a c\rangle^{2}\langle c b\rangle^{2}}, \\
M_{3}^{(0), \alpha^{0}}\left(a^{+}, b^{+}, c^{-}\right) & =\frac{[a b]^{6}}{[a c]^{2}[c b]^{2}}, \\
M_{3}^{(0), \alpha^{0}}\left(a^{+}, b^{+}, c^{+}\right) & =M_{3}^{(0)}\left(a^{-}, b^{-}, c^{-}\right)=0 .
\end{aligned}
$$

These amplitudes have the correct spinor weight and are quadratic in the momenta.

\footnotetext{
${ }^{1}$ We remove a factor of $i(\kappa / 2)^{n-2}$ from the $n$-point amplitude, as set out in (2.19).
} 
For the amplitudes arising from the leading deformation of $\alpha R^{3}$, we must understand the momentum power of the three-point vertex and it's spinor weight. In momentum space $R$ has two powers of momentum ${ }^{2}$ and as such the three-point amplitude should have six powers of momentum. As the graviton is spin two and can be built from the product of two Yang-Mills polarisation vectors, the spinor weight is doubled for each helicity of the graviton with respect to the gluon in Yang-Mills. To be non-trivial, these three-point amplitudes must either be functions of $\langle i j\rangle$ or $[i j]$ exclusively. The simplest polynomial amplitudes with six powers of momenta are

$M_{3}^{(0), \alpha}\left(a^{+}, b^{+}, c^{+}\right)=\alpha[a b]^{2}[b c]^{2}[c a]^{2}, \quad M_{3}^{(0), \alpha}\left(a^{-}, b^{-}, c^{-}\right)=\alpha\langle a b\rangle^{2}\langle b c\rangle^{2}\langle c a\rangle^{2}$

whilst

$$
M_{3}^{(0), \alpha}\left(a^{-}, b^{-}, c^{+}\right)=0, \quad M_{3}^{(0), \alpha}\left(a^{-}, b^{+}, c^{+}\right)=0 .
$$

The helicity amplitudes (5.8) vanish since there are no expressions that satisfy the constraints imposed by spinor and momentum weight. This is essentially the unique choice for a three-point amplitude [45].
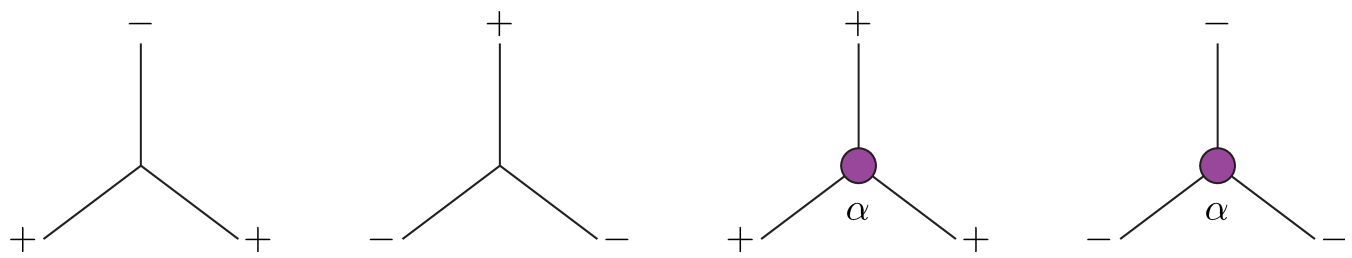

Figure 5.1: The non-zero three-point amplitudes.

The $S$-matrix of the leading deformation of EH gravity in this context must therefore be determined by the $\mathcal{O}(\alpha)$ complex three-point identical helicity amplitudes.

To begin verifying this claim, the next step is to determine the four-point $\mathcal{O}(\alpha)$ amplitudes. This $S$-matrix element is composed of three independent helicity

\footnotetext{
${ }^{1}$ Which can be viewed from noting that the Ricci Scalar in co-ordinate space depends on two spacetime derivatives.
} 


\section{DIAGRAMMAR IN AN EXTENDED THEORY OF GRAVITY}

configurations,

$$
M_{4}^{(0), \alpha}\left(1^{+}, 2^{+}, 3^{+}, 4^{+}\right), \quad M_{4}^{(0), \alpha}\left(1^{-}, 2^{+}, 3^{+}, 4^{+}\right), M_{4}^{(0), \alpha}\left(1^{-}, 2^{-}, 3^{+}, 4^{+}\right) .
$$

These amplitudes are generated by sewing together the $\mathcal{O}\left(\alpha^{0}\right)$ EH amplitudes with the $\mathcal{O}(\alpha)$ amplitudes sourced from the $R^{3}$ term.

In EH gravity, the first two helicity configurations are vanishing, with the MHV amplitude being the only non-trivial amplitude for this multiplicity. However by studying the factorisations, the amplitudes needed to generate the fourpoint MHV at $\mathcal{O}(\alpha)$ are vanishing, with the all-plus and single minus amplitudes being composed of non-vanishing three-point amplitudes.

In order to generate the all-plus and single-minus amplitudes using complex recursion, we must employ the use of a complex shift under which the amplitude vanishes as $|z| \rightarrow \infty$. For the all-plus amplitude, as was the case in all-plus Yang-Mills amplitudes, the Risager shift [37] produces the desired asymptotic behaviour. Whereas the single minus amplitude behaves well under either the Risager shift or the BCFW shift [36] and we are free to choose the shift we prefer. The added bonus of using the BCFW shift is that it does not introduce the reference spinor, $\lambda_{\eta}$, that the Risager shift incorporates. In this sense, it is the preferable choice, since we will need to reuse the result at higher multiplicities and keeping expressions as simple as possible allows for more compact results.

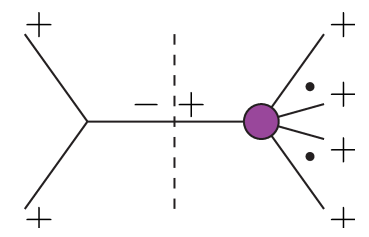

Figure 5.2: General factorisation for an $n$-point $\mathcal{O}(\alpha)$ all-plus amplitude. The $\mathcal{O}(\alpha)$ amplitude can only enter the factorisation on the side of the on-shell propagator with a positive helicity.

The all-plus undergoes a Risager shift, resulting in six factorisations. They come in two types, where the $\mathcal{O}(\alpha)$ appears on either side of the factorisation. 
After performing the residues, which in general for a pole $\langle i j\rangle^{-1}$, becomes,

$$
\operatorname{Res}\left[-\frac{1}{\langle i j\rangle}, z_{I}\right]=1
$$

Two of the types of factorisations are given as,

$$
M^{(0), \alpha^{0}}\left(\hat{c}^{+}, d^{+}, \hat{K}^{-}\right) \frac{1}{s_{c d}} M^{(0), \alpha}\left(\hat{a}^{+}, \hat{b}^{+},-\hat{K}^{+}\right)=\frac{[c d]^{6}}{[c \hat{K}]^{2}[d \hat{K}]^{2}} \frac{1}{s_{c d}}[a b]^{2}[b \hat{K}]^{2}[a \hat{K}]^{2},
$$

and

$$
M^{(0), \alpha}\left(\hat{c}^{+}, d^{+}, \hat{K}^{+}\right) \frac{1}{s_{c d}} M^{(0), \alpha^{0}}\left(\hat{a}^{+}, \hat{b}^{+},+\hat{K}^{+}\right)=\frac{[a b]^{6}}{[b \hat{K}]^{2}[a \hat{K}]^{2}} \frac{1}{s_{c d}}[c d]^{2}[d \hat{K}]^{2}[c \hat{K}]^{2} .
$$

The remaining four factorisations are related to these by considering all the other permutations of the external legs.

Note that the spinor $\bar{\lambda}_{K}$, related to the momentum of the propagator is shifted and still depends on $\lambda_{\eta}$. In general the momentum of the propagator will carry a dependence of the reference spinor $\lambda_{\eta}$. For the shifted legs $\hat{i}, \hat{j}, \hat{k}$, defining the momenta $K$, as the sum of external momenta on the side of the factorisation containing only one of the shifted legs, say leg $i$, we may express the shifted propagator momentum ast,

$$
\left.\hat{\lambda}_{K}=|K| i\right], \quad \hat{\bar{\lambda}}_{K}=\frac{|K| \eta\rangle}{[i|K| \eta\rangle}
$$

In the all-plus case we have shifted $\hat{a}, \hat{b}$ and $\hat{c}$, such that the momentum of the propagator under the Risager shift is given by,

$$
\left.K=\lambda_{K} \bar{\lambda}_{K}, \quad \lambda_{K}=\lambda_{c}[d c]=|d| c\right\rangle, \quad \bar{\lambda}_{K}=\frac{\lambda_{c}\langle c \eta\rangle+\lambda_{d}\langle d \eta\rangle}{[c|d| \eta\rangle}
$$

This shows how the Risager shift incorporates the reference spinor in this case.

\footnotetext{
${ }^{1}$ For completeness, if $-K$ appears in the spinor brackets, then $\bar{\lambda}_{K}$ picks up the minus sign and $\lambda_{K}$ is unaltered. However, since all the spinor brackets in the four-point factorisations are squared, this does not pose a problem here.
} 


\section{DIAGRAMMAR IN AN EXTENDED THEORY OF GRAVITY}

Substituting the values for the shifted propagator into the factorisation and summing over the remaining factorisations 1 , gives a function that is $\lambda_{\eta}$-independent. After some algebra, we arrive at the explicitly $\lambda_{\eta}$-independent function,

$$
M_{4}^{(0), \alpha}\left(a^{+}, b^{+}, c^{+}, d^{+}\right)=10\left(\frac{s t}{\langle a b\rangle\langle b c\rangle\langle c d\rangle\langle d a\rangle}\right)^{2} s t u .
$$

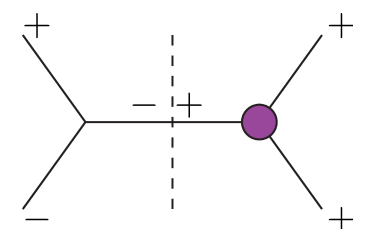

Figure 5.3: The only type of factorisation of the four-point $\mathcal{O}(\alpha)$ single-minus.

The single-minus can be evaluated using the BCFW shift, shifting legs $\hat{a}$ and $\hat{b}$. This excites two factorisations, that take the following form after taking residues,

$$
M^{(0), \alpha^{0}}\left(\hat{a}^{-}, \hat{K}^{-}, d^{+}\right) \frac{1}{s_{a d}} M^{(0), \alpha}\left(\hat{b}^{+}, c^{+},-\hat{K}^{+}\right)=\frac{\langle a \hat{K}\rangle^{6}}{\langle\hat{K} d\rangle^{2}\langle d \hat{a}\rangle^{2}} \frac{1}{s_{a d}}[b c]^{2}[b \hat{K}]^{2}[c \hat{K}]^{2},
$$

and

$$
M^{(0), \alpha^{0}}\left(\hat{a}^{-}, \hat{K}^{-}, c^{+}\right) \frac{1}{s_{a c}} M^{(0), \alpha}\left(\hat{b}^{+}, d^{+},-\hat{K}^{+}\right)=\frac{\langle a \hat{K}\rangle^{6}}{\langle\hat{K} c\rangle^{2}\langle c \hat{a}\rangle^{2}} \frac{1}{s_{a d}}[b d]^{2}[b \hat{K}]^{2}[d \hat{K}]^{2} .
$$

In a similar way to the Risager shift, the BCFW shift affects the momentum of the propagator. In general for the $\mathrm{BCFW}$ shift $[j, i\rangle$, we define the momenta $K$, as the sum of external momenta on the side of the factorisation $\lambda_{i}$. Given this

\footnotetext{
${ }^{1}$ appropriately redefining the shifted propagator
} 
we may write日,

$$
\left.\hat{\lambda}_{K}=|K| i\right], \quad \hat{\bar{\lambda}}_{K}=\frac{|K| j\rangle}{[i|K| j\rangle}
$$

Applying this to the factorisations above and summing, reduces to,

$$
M_{4}^{(0), \alpha}\left(a^{-}, b^{+}, c^{+}, d^{+}\right)=\left(\frac{[b d]^{2}}{[a b]\langle b c\rangle\langle c d\rangle[d a]}\right)^{2} \frac{s^{3} t^{3}}{u} .
$$

The other non-trivial amplitudes are related to these results by parity conjugation.

These four-point amplitudes due to a $R^{3}$ term are not a novel result. They were previously computed using field theory methods [168]. Our results agree with the previously calculated expressions. They have also been found recursively using an all-line shift [135], a technique where all legs have shifted momenta. Additionally, they vanish to all orders in a supersymmetric theory: a fact used to show supergravity was two-loop ultraviolet finite [169, 170]. In a similar vein, they also appear as the UV counterterms of both two-loop gravity in four dimensions [164, 171] and one-loop gravity in six dimensions [172].

The five-point $\mathcal{O}(\alpha)$ amplitudes follow on naturally. Using the four-point and three-point amplitudes from the extended theory and EH gravity we construct the following independent five-point amplitudes.

$$
M_{5}^{(0), \alpha}\left(a^{+}, b^{+}, c^{+}, d^{+}, e^{+}\right), \quad M_{5}^{(0), \alpha}\left(a^{-}, b^{+}, c^{+}, d^{+}, e^{+}\right), \quad M_{5}^{(0), \alpha}\left(a^{-}, b^{-}, c^{+}, d^{+}, e^{+}\right) .
$$

As before, the shift (5.5) yields an all-plus amplitude that is independent of $\lambda_{\eta}$ and has full crossing symmetry:

$$
M_{5}^{(0), \alpha}\left(a^{+}, b^{+}, c^{+}, d^{+}, e^{+}\right)=\left(\sum_{P_{6}} T_{(a, b, c),(d, e)}^{A}+\sum_{Z_{3}} T_{(a, b, c),(d, e)}^{B}\right)
$$

${ }^{1}$ Again, for completeness, if $-K$ appears in the spinor brackets, then $\bar{\lambda}_{K}$ picks up the minus sign and $\lambda_{K}$ is unaltered. However, since all the spinor brackets in the four-point factorisations are squared, this does not pose a problem here. 


\section{DIAGRAMMAR IN AN EXTENDED THEORY OF GRAVITY}

where

$$
\begin{gathered}
T_{(a, b, c),(d, e)}^{A}=10 \frac{[a d]}{\langle a d\rangle} \frac{[e c][e b]}{\langle a \eta\rangle^{2}\langle d \eta\rangle} \frac{[b c]^{2}}{\langle d e\rangle} \times\left[e\left|K_{a d}\right| \eta\right\rangle\left[b\left|K_{a d}\right| \eta\right\rangle\left[c\left|K_{a d}\right| \eta\right\rangle \\
T_{(a, b, c),(d, e)}^{B}=-10 \frac{[a d][a e][b c]\left[a\left|K_{b c}\right| \eta\right\rangle^{2}\left[e\left|K_{b c}\right| \eta\right\rangle\left[d\left|K_{b c}\right| \eta\right\rangle}{\langle b c\rangle\langle b \eta\rangle^{2}\langle c \eta\rangle^{2}} \frac{[d e]}{\langle d e\rangle}
\end{gathered}
$$

and $Z_{3}$ denotes summation over the three cyclic permutations of legs $a, b$ and $c$. $P_{6}=Z_{3} \times Z_{2}$ denotes the three permutations of $\{a, b, c\}$ under $Z_{3}$ together with interchange of legs $d$ and $e$. Although $\lambda_{\eta}$ appears explicitly in the expression for the amplitude, $M_{5}^{(0), \alpha}\left(a^{+}, b^{+}, c^{+}, d^{+}, e^{+}\right)$is independent of $\lambda_{\eta}$, however this has not been made manifest.
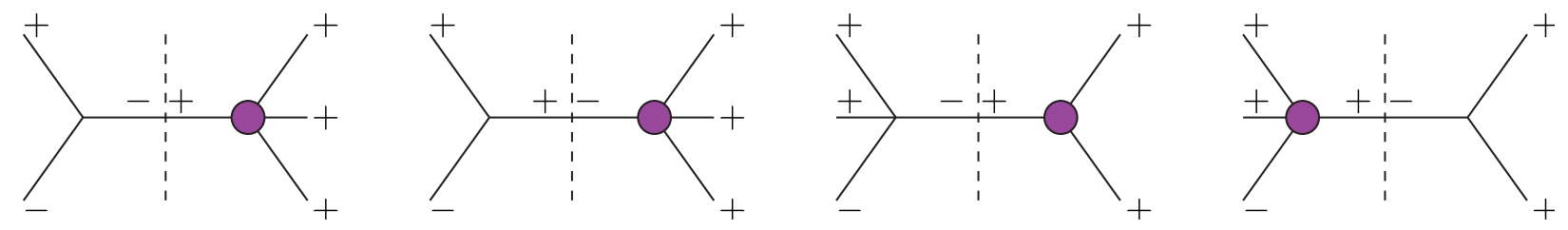

Figure 5.4: Factorisations of the five-point single-minus amplitude.

There are many more factorisations of the single minus amplitude since adding an additional negative external helicity allows for more internal helicity configurations, as shown in fig. 5.4. Using the BCFW shift on $\left(\bar{\lambda}_{a}, \lambda_{b}\right)$ we obtain the amplitude,

$$
\begin{aligned}
& M_{5}^{(0), \alpha}\left(a^{-}, b^{+}, c^{+}, d^{+}, e^{+}\right)=\frac{10}{[a b]^{2}}\left(\prod_{i, j=b, c, d, e, i<j}[i j]\right)\left(\frac{\langle a e\rangle}{[a e]} \frac{[b e]^{3}}{\langle c d\rangle}+\frac{\langle a c\rangle}{[a c]} \frac{[b c]^{3}}{\langle d e\rangle}+\frac{\langle a d\rangle}{[a d]} \frac{[b d]^{3}}{\langle e c\rangle}\right) \\
& +\frac{\langle a b\rangle^{2}}{\langle c d\rangle\langle c e\rangle\langle d e\rangle \prod_{i=c, d, e}\langle a i\rangle}\left(\frac{[b c]^{5}[d e]\langle a c\rangle^{5}}{\langle b c\rangle}+\frac{[b d]^{5}[e c]\langle a d\rangle^{5}}{\langle b d\rangle}+\frac{[b e]^{5}[c e]\langle a e\rangle^{5}}{\langle b e\rangle}\right) \\
& +\frac{1}{\langle a b\rangle^{2}\langle c d\rangle\langle c e\rangle\langle d e\rangle}\left(\frac{[b c][d e]^{5}\langle a e\rangle^{3}\langle a d\rangle^{3}}{\langle b c\rangle}+\frac{[b d][e c]^{5}\langle a c\rangle^{3}\langle a e\rangle^{3}}{\langle b d\rangle}\right. \\
& \left.+\frac{[b e][c d]^{5}\langle a d\rangle^{3}\langle a c\rangle^{3}}{\langle b e\rangle}\right) .
\end{aligned}
$$


The five-point MHV amplitude is non-zero since we have sufficient external legs to include a non-zero $\mathcal{O}(\alpha)$ tree. The non-zero factorisations of the amplitude are shown in fig. 5.5.
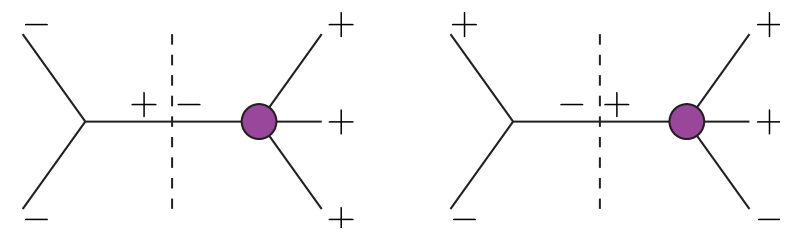

Figure 5.5: Factorisations of the five-point MHV amplitude

This amplitude can be obtained using the $[-,-\rangle$ or $[-,+\rangle$ BCFW shifts. We employ the shift on the two negative helicity legs as this excites only the second factorisation of fig. 5.5 hence reducing the computational workload. The recursion generates the following expression,

$$
\begin{aligned}
& M_{5}^{(0), \alpha}\left(a^{-}, b^{-}, c^{+}, d^{+}, e^{+}\right)=-s_{c d} \frac{\langle a e\rangle}{[a e]} \frac{[c d]^{2}[c e]^{3}[d e]^{3}}{[a b]^{2}[b c][b d]} \\
& -s_{d e} \frac{\langle a c\rangle}{[a c]} \frac{[d e]^{2}[d c]^{3}[e c]^{3}}{[a b]^{2}[b d][b e]}-s_{e c} \frac{\langle a d\rangle}{[a d]} \frac{[e c]^{2}[e d]^{3}[c d]^{3}}{[a b]^{2}[b e][b c]}
\end{aligned}
$$

With this, all the independent five-point amplitudes contributing to the treelevel $S$-matrix of this extended theory of gravity have been determined. The process can be continued and we have calculated the $\mathcal{O}(\alpha)$ amplitudes up to $n=8$ solely using complex recursion and the two types of complex shifts. The amplitudes have the correct symmetries, are $\eta$-independent and have the correct leading soft-limits. Since the all-plus amplitudes of higher multiplicity have not been made manifestly independent of $\lambda_{\eta}$ and the expressions for the remaining helicity amplitudes grow in size considerably, the remaining helicity amplitudes $M_{n}^{(0), \alpha}$ for $n \leq 7$ are available in Mathematica format at http://pyweb.swan.ac.uk/ dunbar/Smatrix.html.

In ref. 173 amplitudes in Yang-Mills theory extended by $F^{3}$ terms were studied. Then using double copy techniques and the KLT relations [123] graviton scattering amplitudes were derived up to $n=6$. As noted in [173 these correspond to amplitudes in a $R+\alpha R^{3}+\sqrt{\alpha} R^{2} \phi$ theory. The four-point amplitudes 


\section{DIAGRAMMAR IN AN EXTENDED THEORY OF GRAVITY}

in the $R+\alpha R^{3}+\sqrt{\alpha} R^{2} \phi$ and the $R^{3}$ theories are proportional [135, 173] but beyond four-point the two sets of amplitudes are functionally different. The all-plus amplitude in the two theories remain proportional for $n>4$ with

$$
M_{n}^{(0), R^{3}+R^{2} \phi}\left(1^{+}, 2^{+}, \cdots n^{+}\right)=\frac{5}{2} M_{n}^{(0), R^{3}}\left(1^{+}, 2^{+}, \cdots n^{+}\right),
$$

and we find this is the case for $n \leq 7$.

\subsection{Soft Limits}

Graviton scattering amplitudes are singular as an external graviton becomes soft. Parametrising the momentum of the $n$-th leg as $k_{n}^{\mu}=t \times k_{s}^{\mu}$ then in the limit $t \longrightarrow 0$ the singularity in the $n$-point amplitude is,

$$
M_{n} \longrightarrow \frac{1}{t} \times S^{(0)} \times M_{n-1}+O\left(t^{0}\right)
$$

where $M_{n-1}$ is the $(n-1)$-point amplitude and $S^{(0)}$ is the universal leading soft factor.

By sending the momentum of a positive helicity leg to become soft using the following parametrisation,

$$
\lambda_{n}=t \times \lambda_{s}, \quad \bar{\lambda}_{n}=\bar{\lambda}_{s}
$$

and sending $t \longrightarrow 0$ we see that the amplitude has $t^{-3}$ singularities in complex momenta. At tree-level the amplitudes behave as (5.29), given by soft theorems [115] in this limit. The coefficient of this leading singularity is the universal leading soft factor determined by Weinberg. It has also been proposed [114 116 that the sub-leading and sub-sub-leading terms are also universal.

$$
M_{n}^{\text {tree }}=S_{t} M_{n-1}^{\text {tree }}+O\left(t^{0}\right)=\left(\frac{1}{t^{3}} S^{(0)}+\frac{1}{t^{2}} S^{(1)}+\frac{1}{t} S^{(2)}\right) M_{n-1}^{\text {tree }}+O\left(t^{0}\right)
$$


where, for a positive helicity-leg becoming soft 115, 174, 175

$$
\begin{aligned}
S^{(0)} & =-\sum_{i=1}^{n-1} \frac{[s i]\langle i \alpha\rangle\langle i \beta\rangle}{\langle s i\rangle\langle s \alpha\rangle\langle s \beta\rangle}, \\
S^{(1)} & =-\frac{1}{2} \sum_{i=1}^{n-1} \frac{[s i]}{\langle s i\rangle}\left(\frac{\langle i \alpha\rangle}{\langle s \alpha\rangle}+\frac{\langle i \beta\rangle}{\langle s \beta\rangle}\right) \bar{\lambda}_{s}^{\dot{a}} \frac{\partial}{\partial \bar{\lambda}_{i}^{\dot{a}}}, \\
S^{(2)} & =\frac{1}{2} \sum_{i=1}^{n-1} \frac{[i s]}{\langle i s\rangle} \bar{\lambda}_{s}^{\dot{a}} \bar{\lambda}_{s}^{\dot{b}} \frac{\partial}{\partial \bar{\lambda}_{i}^{\dot{a}}} \frac{\partial}{\partial \bar{\lambda}_{i}^{\dot{b}}} .
\end{aligned}
$$

The proof of the soft theorems follows from Ward identities of extended Bondi, van der Burg, Metzner and Sachs (BMS) symmetry [176]. Although exact for tree level amplitudes, these subleading theorems receive loop corrections [83, 174, 177. Whether the soft theorems extend beyond EH gravity has been examined before. In particular the leading soft behaviour can often be used as a check upon amplitudes such, e.g. in [173]. The leading and sub-leading limits were shown to hold for a $R^{3}$ insertion in [178]. Here we examine the amplitudes and, in particular, test the sub-sub-leading soft behaviour.

We can summarise the behaviour of the leading amplitudes, $M_{n}^{(1)}$, simply by stating:

\section{All the amplitudes calculated satisfy the soft limits of (5.29) up to and including the sub-sub-leading term.}

We have verified this for all helicity amplitudes up to $n=8$. Note: to check (5.29) one must implement momentum conservation consistently between the $n$-point amplitudes and the $(n-1)$-point amplitudes which in essence specifies how the point $t=0$ is approached. These are several ways to do this. We have followed the prescription of [115] by Laurent expanding the amplitude around $t=0$ and numerically evaluating the amplitude checking the coefficients of the terms $t^{-n}$ where $n=1,2,3$.

Incidentally as a consequence of eq. (5.26) the amplitude $M_{n}^{(1), R^{3}+R^{2} \phi}\left(1^{+}, 2^{+}, \cdots n^{+}\right)$ also satisfies the soft theorems to sub-sub leading level. 


\section{DIAGRAMMAR IN AN EXTENDED THEORY OF GRAVITY}

\subsection{Other Theories}

We have chosen to extend gravity using a three-point vertex and use a diagrammar approach only considering massless gravity, i.e. on-shell amplitudes. There is, of course, complementarity between this approach and that of Lagrangian based field theory. The single choice of three-point amplitude corresponds to the single $R^{3}$ field density that affects on-shell amplitudes. As there is only one choice of $R^{3}$ that can contribute, this makes the extended $S$-matrix simply depend upon the single parameter $\alpha$.

If we were to deform EH gravity by an additional four-point amplitude then there are more choices consistent with symmetry and spinor weight, e.g. we could have

$$
\begin{aligned}
M_{4}\left(a^{+}, b^{+}, c^{+}, d^{+}\right) & =\alpha_{1} \quad\left(\langle a b\rangle^{4}\langle c d\rangle^{4}+\langle a c\rangle^{4}\langle b d\rangle^{4}+\langle a d\rangle^{4}\langle b c\rangle^{4}\right) \\
& +\alpha_{2} \quad(\langle a b\rangle\langle b c\rangle\langle c d\rangle\langle d a\rangle+\text { permutations })^{2}+\cdots
\end{aligned}
$$

From a field theory perspective this freedom corresponds to the observation that there are multiple $R^{4}$ tensors that contribute to on-shell amplitudes [179].

The same issue arises when we consider the further expansion in $\alpha$. If we consider $M_{4}^{(0) \alpha^{2}}\left(a^{-}, b^{-}, c^{+}, d^{+}\right)$there is a single factorisation as shown in fig. 6.3. The amplitude,

$$
M_{4}^{(2)}\left(a^{-}, b^{-}, c^{+}, d^{+}\right)=\langle a b\rangle^{4}[c d]^{4}\left(\frac{t u+\beta s^{2}}{s}\right)
$$

has the correct factorisation for any choice of $\beta$. This ambiguity means we also have to specify the four-point amplitude in order to determine the $S$-matrix. In the diagrammar approach, this ambiguity arises due to the existence of a polynomial function with the correct symmetries and spinor and momentum weight. From a field theory perspective, additional counterterms can contribute to this amplitude. Specifically, we could deform the theory via

$$
R \longrightarrow R+C_{\alpha} R^{3}+C_{\beta} D^{2} R^{4}
$$


and the four-point amplitude is only specified once $C_{\alpha}$ and $C_{\beta}$ are determined.

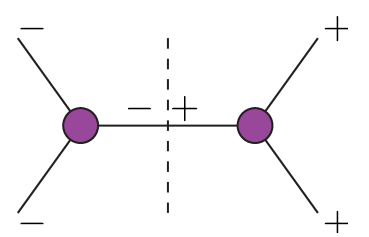

Figure 5.6: Factorisations of the four-point MHV amplitude at $\alpha^{2}$.

\subsection{Conclusion}

We have constructed the tree-level $S$-matrix elements of the leading deformation to EH gravity arising in an extended theory of gravity. There is only one choice of $R^{3}$ that affects on-shell amplitudes, and we fix the form of the three-point amplitudes arising from this extension through spinor and momentum weight for massless gluons. Therefore, the theory is extended by the addition of amplitudes which are polynomial in momentum, thus implicitly imposing locality and unitarity on the $S$-matrix. With this starting point, the $S$-matrix has been constructed from the two types of three-point amplitudes contributing to the $\mathcal{O}(\alpha)$ deformation in addition to demanding factorisation on propagator poles. In 45 the authors call such theories fully constructible, hence we find the $\mathcal{O}(\alpha)$ extended theory to be such a theory.

Beyond the leading deformation, there exist independent polynomial amplitudes with a higher number of legs and these must be specified in order to fully determine the $S$-matrix. Consistency of this approach and a field theoretic approach beyond leading order, requires a correspondence between these polynomial amplitudes and the counter terms contributing to on-shell amplitudes.

In addition to constructing the $S$-matrix of this extended theory of gravity, we find that these amplitudes satisfy the same soft theorems as the tree amplitudes of EH gravity up to and including the sub-subleading terms.

In the context of perturbation theory, this is only the leading order term. Armed with the understanding of the tree-level $S$-matrix elements for this extended theory, we are able to understand the renormalisability of this theory by 


\section{DIAGRAMMAR IN AN EXTENDED THEORY OF GRAVITY}

examining the ultraviolet behaviour of the one-loop amplitudes. Given the known

basis of integral functions that describe a one-loop amplitude, investigation into the UV behaviour can be achieved by considering the double-cuts of the amplitudes and uncovering the coefficients of the scalar bubble integral functions. In doing so we understand the form of the counterterms that are required in order to renormalise the extended theory of gravity. 


\section{Chapter 6}

\section{Loop Amplitudes in an Extended Theory of Gravity}

\subsection{Introduction}

In the previous chapter we investigated the tree-level $S$-matrix of Einstein-Hilbert (EH) gravity extended by the inclusion of an $\alpha R^{3}$ term. The amplitudes that compose the $S$-matrix of this theory can be expanded as a power series in $\alpha$,

$$
M_{n}(1, \cdots, n)=\sum_{r=0} \alpha^{r} M_{n}^{(L), \alpha^{r}}(1, \cdots, n)
$$

where $L$ is the number of loops and $M_{n}^{(0), \alpha^{0}}$ is the EH tree-level gravity amplitude. The theory we are considering would arise using field theory methods from the Lagrangian

$$
L=\int d^{D} x \sqrt{-g}\left(R+C_{\alpha} R_{a b c d} R^{c d e f} R_{e f}^{a b}\right),
$$

where $C_{\alpha}=\alpha / 60$. We focused on leading deformation of the theory, i.e. the $r=1$ part of the extended theory.

In conjunction with information available from the Lagrangian perspective, we used complex momenta and spinor weight to constrain the form of the threepoint amplitudes. By employing the use of complex recursion and factorisation theorems involving on-shell amplitudes we were able to determine the tree-level $S$-matrix [165] for the $\mathcal{O}(\alpha)$ deformation of this extended theory of gravity and 


\section{LOOP AMPLITUDES IN AN EXTENDED THEORY OF GRAVITY}

label it fully constructible [45]. This is akin to the philosophy of the S-matrix program of the 1960's [46].

Having understood that the $\mathcal{O}(\alpha)$ tree-level $S$-matrix is fully constructible, it is an interesting question to investigate whether the theory itself is renormalisable at the one-loop level in perturbation theory. In particular what counterterms are required to renormalise the theory. As we have done with the tree-level case of gravitational theory, we may borrow from the lessons we have learned in the context of Yang-Mills amplitudes. In particular, we use the knowledge that a Passarino-Veltman reduction [52] can be applied to the one-loop Feynman integral functions that are present in this extended theory of gravity to construct a basis of integrals. The singularity structure of one-loop amplitudes are also well understood and we may reorganise the amplitude according to this singularity structure. The $\mathcal{O}(\alpha)$ tree-level amplitudes we have calculated in [112] and shown in the previous chapter, can be coupled with four-dimensional unitarity to understand the UV structure of the gravity amplitudes at one-loop.

This chapter is based on the work performed in [113 in which we examine the one-loop amplitudes for both extended Yang-Mills and gravity - in particular their UV structure - using four-dimensional unitarity. In agreement with long established results we find that the extended Yang-Mills theory is renormalised 180 183 through a redefinition of the coupling constants. For the extended theory of gravity however, we find that additional counter-terms, written in the spinor helicity, are required in order to renormalise the theory. The use of four-dimensional unitarity techniques gives relatively easy access to the UV structures of these theories.

\subsection{Structure of the Amplitudes}

Before determining the one-loop amplitudes in these extended theories, we take a short moment to review the general analytic form of one-loop amplitudes in massless gauge theories. A one-loop amplitude in a theory of massless particles 
can be expressed, after a Passarino-Veltman reduction [52], in the form

$$
A_{n}^{(1)}=\sum_{i \in \mathcal{C}} a_{i} I_{4}^{i}+\sum_{j \in \mathcal{D}} b_{j} I_{3}^{j}+\sum_{k \in \mathcal{E}} c_{k} I_{2}^{k}+R_{n}+O(\epsilon)
$$

where the $I_{m}^{i}$ are $m$-point scalar integral functions and the $a_{i}$ etc. are rational coefficients. $\mathcal{C}$ is the set of box integral functions with all allowed partitions of the external legs between the corners. For a colour-ordered Yang-Mills amplitude the allowed partitions respect the cyclic ordering of the legs, whereas for a gravity theory, all possible permutations are allowed. Similarly $\mathcal{D}$ and $\mathcal{E}$ are the sets of triangle and bubble integral functions. The sums include all possible integral functions allowed by the ordering and depend upon the masses of the diagrams, i.e. the number vertices of a diagram with a non-null sum of external momenta. $R_{n}$ is a purely rational term.

Alternately, we can re-express the one-loop amplitude for pure Yang-Mills or gravity in a form which highlights the singular structure of the amplitude,

$$
A_{n}^{(1)}=A_{n}^{(0)} I_{n}+G_{n}+F_{n}+R_{n}
$$

where $I_{n}$ contains the soft-singular Infra-Red (IR) [32] terms of the amplitude and is

$$
I_{n}=-\frac{c_{\Gamma}}{(4 \pi)^{2}} \sum_{i=1}^{n} \frac{\left(-s_{i i+1} / \mu^{2}\right)^{-\epsilon}}{\epsilon^{2}},
$$

for a leading in colour gluon amplitude where $c_{\Gamma}=(4 \pi)^{\epsilon} \Gamma^{2}(1-\epsilon) \Gamma(1+\epsilon) / \Gamma(1-2 \epsilon)$. For a graviton scattering amplitude [117, 184,

$$
I_{n}=-\frac{c_{\Gamma}}{(4 \pi)^{2}} \sum_{i<j} s_{i j} \frac{\left(-s_{i j} / \mu^{2}\right)^{-\epsilon}}{\epsilon^{2}} .
$$

Within the decomposition of (6.3) the contributions to $I_{n}$ arise from the box integral functions and the one and two mass triangle integral functions. $G_{n}$ is of the form

$$
G_{n}=\sum_{i} c_{i} \frac{\left(-s_{i i+1} / \mu^{2}\right)^{-\epsilon}}{\epsilon}
$$




\section{LOOP AMPLITUDES IN AN EXTENDED THEORY OF GRAVITY}

for leading in colour gluon scattering amplitudes and

$$
G_{n}=\sum_{i<j} c_{i j} \frac{\left(-s_{i j} / \mu^{2}\right)^{-\epsilon}}{\epsilon}
$$

for graviton scattering. Within the integral basis decomposition (6.3), the $G_{n}$ arise from the bubble integral functions. The $G_{n}$ terms contain both the collinear IR singular terms and the UV divergences. The function $F_{n}$ contains the finite transcendental functions. These arise from both the box integral functions and from the three-mass triangle integral function. $R_{n}$ is the remaining rational term finite in the dimensional regularisation parameter $\epsilon$.

The coefficients of the integral functions can be determined using four dimensional generalised unitarity techniques from the on-shell amplitudes. Computing $R_{n}$ from unitarity requires using $d=4-2 \epsilon$ tree amplitudes.

We will find that the form of the IR singularities is not altered in the extended theories, as is expected by naive power counting. That is, since the additional vertices introduced by the extensions have a larger momentum power counting they do not affect the $\left(-\mu^{2} / s\right)^{\epsilon}$ IR singularities.

\subsection{Yang-Mills Case}

Before looking at gravity theories, we consider the case of gluon scattering in pure Yang-Mills. We consider the leading in $N_{c}$ colour partial amplitude that have fixed cyclic ordering. The full amplitude can be reconstructed by reintroducing the trace structures and deducing the subleading partial amplitudes through decoupling identities [128].

We define the theory in an analogous fashion to the previous chapter - from the fundamental complex three-point amplitudes,

$$
A_{3}^{(0)}\left(1^{h_{1}}, 2^{h_{2}}, 3^{h_{3}}\right)
$$

where $h_{i}$ is the helicity of the $i$-th leg. For gluons the helicity is \pm 1 . The constraints imposed from spinor weight and momentum weight give the following 
four allowed tree-level amplitudes 1 ,

$$
\begin{aligned}
& A_{3}^{(0), \alpha^{0}}\left(1^{-}, 2^{-}, 3^{+}\right)=g \frac{\langle 12\rangle^{3}}{\langle 23\rangle\langle 31\rangle}, \\
& A_{3}^{(0), \alpha^{0}}\left(1^{+}, 2^{+}, 3^{-}\right)=g \frac{[21]^{3}}{[23][31]}, \\
& A_{3}^{(0), \alpha}\left(1^{+}, 2^{+}, 3^{+}\right)=\alpha g[12][23][31], \\
& A_{3}^{(0), \alpha}\left(1^{-}, 2^{-}, 3^{-}\right)=\alpha g\langle 13\rangle\langle 32\rangle\langle 21\rangle .
\end{aligned}
$$

The first two amplitudes are the well known MHV ("Maximally Helicity Violating") and $\overline{\mathrm{MHV}}$ amplitudes. The parameter $\alpha$ is dimensionful with mass dimension minus two, ie. $\alpha=c / M^{2}$ where $c$ is dimensionless in natural units and $M$ is some mass scale日 and the $\alpha$ expansion can be considered as an expansion in inverse powers of the mass $M$.

In this section we expand Yang-Mills theory by including the $\alpha$-vertices in addition to the MHV vertices. The amplitudes in this theory can then be expanded as a power series in $\alpha$,

$$
A_{n}(1, \cdots, n)=\sum_{r=0} \alpha^{r} A_{n}^{\alpha^{r}}(1, \cdots, n)
$$

where $A_{n}^{\alpha^{0}}$ is the usual Yang-Mills amplitude.

From a Lagrangian field theory viewpoint we are extending the theory by

$$
\mathcal{L}_{F^{3}}=\alpha^{\prime} \operatorname{Tr}\left(F_{\mu \nu} F^{\nu \rho} F_{\rho}{ }^{\mu}\right)
$$

where $\alpha^{\prime}=-\alpha g / 3[173]$. Having defined the three-point vertices, factorisation theorems can be used [112, 135] to obtain the leading four-point tree amplitudes

\footnotetext{
${ }^{1}$ We reintroduce the couplings just for this definition. Subsequently, we remove a factor of $g$.

${ }^{2}$ With the normalisation of $(6.10)$ each $n$-point, $L$-loop amplitude contains a factor of $g^{n-2+2 L}$ which we suppress.
} 


\section{LOOP AMPLITUDES IN AN EXTENDED THEORY OF GRAVITY}

in an analogous way as described in chapter 5 :

$$
\begin{aligned}
& A_{4}^{(0), \alpha}\left(1^{+}, 2^{+}, 3^{+}, 4^{+}\right)=2 \alpha \frac{s t u}{\langle 12\rangle\langle 23\rangle\langle 34\rangle\langle 41\rangle}=2 \alpha K_{++++} \times u \\
& A_{4}^{(0), \alpha}\left(1^{-}, 2^{+}, 3^{+}, 4^{+}\right)=-\alpha \frac{[24]^{2} s t}{[12]\langle 23\rangle\langle 34\rangle[41]}=\alpha K_{-+++} \times u .
\end{aligned}
$$

These expressions match those obtained from Feynman diagram calculations 185, or colour-kinematics duality [173] or scattering equations [186]. The combinations $K_{++++}$and $K_{-+++}$carry all the necessary spinor weight of the amplitude with $\left|K_{++++}\right|=\left|K_{-+++}\right|=1$ for real momenta. The factor $K_{++++}$has manifest cyclic symmetry but is also fully crossing symmetric since

$$
\frac{s t}{\langle 12\rangle\langle 23\rangle\langle 34\rangle\langle 41\rangle}=\frac{s u}{\langle 12\rangle\langle 24\rangle\langle 43\rangle\langle 31\rangle}=\frac{t u}{\langle 14\rangle\langle 42\rangle\langle 23\rangle\langle 31\rangle} \text {. }
$$

Similarly $K_{-+++}$has manifest flip-symmetry but is also invariant under exchange of the positive helicity legs, $2 \leftrightarrow 3$ etc. These factors can be written in many ways, e.g.

$$
K_{++++}=-\frac{[12]^{2}}{\langle 34\rangle^{2}}=-\frac{[12]^{2}[34]^{2}}{s^{2}} .
$$

For the pure Yang-Mills theory, all tree amplitudes can be constructed using factorisation from the three-point trees [36] i.e. the theory is "constructible" using the definition of [45]. The tree-amplitudes arising from the leading deformation, $A_{n}^{(0), \alpha}$, can also be constructed in this way, however amplitudes beyond this leading deformation are not constructible purely from factorisation. This will be pursued further in the context of gravity theories later.

We now wish to determine the one-loop amplitudes in the extended theory to $\mathcal{O}(\alpha)$. Unitarity methods have proven very efficient in determining one-loop amplitudes using the on-shell tree amplitudes.

Working in four-dimensions, the simplicity of the four-dimensional trees greatly simplifies the calculation of the coefficients of the integral functions, but does not allow us to compute the finite rational terms $R$. Since we are mainly interested in the UV singularities, which come with an accompanying $\ln \left(\mu^{2} / s\right)$, four dimensional two-particle cuts suffice [187]. 
Noting that

$$
\left.A_{4}^{(1), \alpha}\left(1^{-}, 2^{-}, 3^{+}, 4^{+}\right)\right|_{\text {cut part }}=0
$$

since there are no non-vanishing four dimensional cuts for these amplitudes, the non-vanishing $\mathcal{O}(\alpha)$ amplitudes are the all-plus and the single minus.

Calculating the $s$-channel two-particle cut for the all-plus amplitude, as shown in fig.6.1, gives

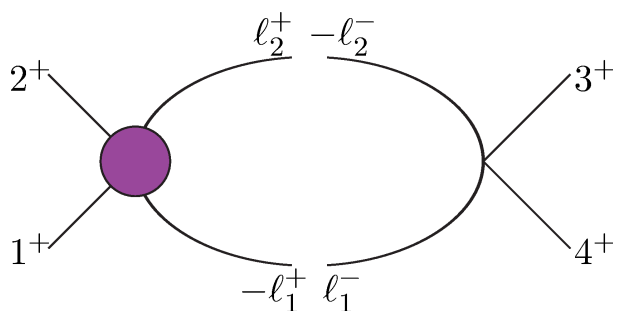

Figure 6.1: The two-particle cut of the all-plus amplitude.

$$
\begin{aligned}
C_{2} & =\int d \operatorname{LIPS} A_{4}^{(0), \alpha}\left(1^{+}, 2^{+}, \ell_{2}^{+},-\ell_{1}^{+}\right) \times A_{4}^{(0), \alpha^{0}}\left(\ell_{1}^{-},-\ell_{2}^{-}, 3^{+}, 4^{+}\right) \\
& =-2 \alpha \int d \operatorname{LIPS} \frac{s_{12} s_{1 \ell_{1}} s_{1 \ell_{2}}}{\langle 12\rangle\left\langle 2 \ell_{2}\right\rangle\left\langle\ell_{2} \ell_{1}\right\rangle\left\langle\ell_{1} 1\right\rangle} \times \frac{\left\langle\ell_{1} \ell_{2}\right\rangle^{4}}{\langle 34\rangle\left\langle 4 \ell_{1}\right\rangle\left\langle\ell_{1} \ell_{2}\right\rangle\left\langle\ell_{2} 3\right\rangle} \\
& =-2 \alpha \int d \operatorname{LIPS} \frac{s_{12}}{\langle 12\rangle\langle 34\rangle} \frac{s_{1 \ell_{2}}\left[\ell_{1} 1\right]}{\left\langle 2 \ell_{2}\right\rangle} \times \frac{\left\langle\ell_{1} \ell_{2}\right\rangle^{2}}{\left\langle 4 \ell_{1}\right\rangle\left\langle\ell_{2} 3\right\rangle},
\end{aligned}
$$

where we also have a configuration where the $\alpha$ vertex is on the right hand side. Manipulating eq. (6.17) using

$$
\frac{\left\langle\ell_{2} \ell_{1}\right\rangle\left[\ell_{1} 1\right]}{\left\langle 2 \ell_{2}\right\rangle}=\frac{\left\langle\ell_{2} 2\right\rangle[21]}{\left\langle 2 \ell_{2}\right\rangle}=[12]
$$

we have

$$
C_{2}=-2 \alpha \int d \operatorname{LIPS} \frac{[12]^{2}}{\langle 34\rangle} \times \frac{s_{1 \ell_{2}}\left\langle\ell_{1} \ell_{2}\right\rangle}{\left\langle 4 \ell_{1}\right\rangle\left\langle\ell_{2} 3\right\rangle} .
$$




\section{LOOP AMPLITUDES IN AN EXTENDED THEORY OF GRAVITY}

Now

$$
\left\langle 4 \ell_{1}\right\rangle\left\langle\ell_{2} 3\right\rangle \times\left[\ell_{1} \ell_{2}\right]=\left\langle 4\left|\ell_{1} \ell_{2}\right| 3\right\rangle=-\langle 43\rangle\left[3\left|\ell_{2}\right| 3\right\rangle=-\langle 34\rangle\left(\ell_{2}-k_{3}\right)^{2},
$$

SO

$$
C_{2}=2 \alpha \int d \operatorname{LIPS} \frac{[12]^{2} s_{12}}{\langle 34\rangle^{2}} \times \frac{\left[1\left|\ell_{2}\right| 1\right\rangle}{\left(\ell_{2}-k_{3}\right)^{2}}
$$

Rather than perform the integration over the cut momenta, we recognise this as the cut of a covariant integral which we can evaluate

$$
\int d \operatorname{LIPS} f\left(\ell_{1}, \ell_{2}\right) \longrightarrow \int d^{D} \ell \frac{f\left(\ell_{1}, \ell_{2}\right)}{\ell_{1}^{2} \ell_{2}^{2}}
$$

where we only keep terms with a $s$-channel cut in the resultant integral [53, 56]. Consequently we replace $C_{2}$ by a triangle integral with linear numerator $I_{3}^{1 m}\left[\ell_{2}^{\mu}\right]$. Using

$$
I_{3}^{1 m}\left[\ell_{2}^{\mu}\right]=\frac{\left(k_{4}-k_{3}\right)^{\mu}}{s} I_{2}^{2 m}(s)+k_{3}^{\mu} I_{3}^{1 m}(s)
$$

where $I_{2}^{2 m}(s)$ is the scalar bubble integral function and $I_{3}^{1 m}(s)$ is the one-mass scalar triangle integral function which only depend on the kinematic variable $s$, we obtain

$$
\begin{aligned}
& -2 \alpha \frac{[12]^{2}}{\langle 34\rangle^{2}}[1|(4-3)| 1\rangle I_{2}^{2 m}(s)-2 \alpha \frac{[12]^{2} s_{12}}{\langle 34\rangle^{2}}[1|3| 1\rangle I_{3}^{1 m}(s) \\
= & -2 \alpha \frac{[12]^{2}}{\langle 34\rangle^{2}}(t-u) I_{2}^{2 m}(s)-2 \alpha \frac{[12]^{2} s_{12} s_{13}}{\langle 34\rangle^{2}} I_{3}^{1 m}(s) \\
= & 2 \alpha K_{++++}(t-u) I_{2}^{2 m}(s)+2 \alpha K_{++++} u \times s I_{3}^{1 m}(s) .
\end{aligned}
$$

Doubling this to account for inserting the $F^{3}$ operator on the opposite side of the cut and combining with the $t$-channel cut leads to the amplitude

$$
\begin{aligned}
A_{4}^{(1), \alpha}\left(1^{+}, 2^{+}, 3^{+}, 4^{+}\right) & =A_{4}^{(0), \alpha} I_{4} \\
+4 \alpha K_{++++} & {\left[(t-u) I_{2}^{2 m}(s)+(s-u) I_{2}^{2 m}(t)\right]+R_{4}, }
\end{aligned}
$$



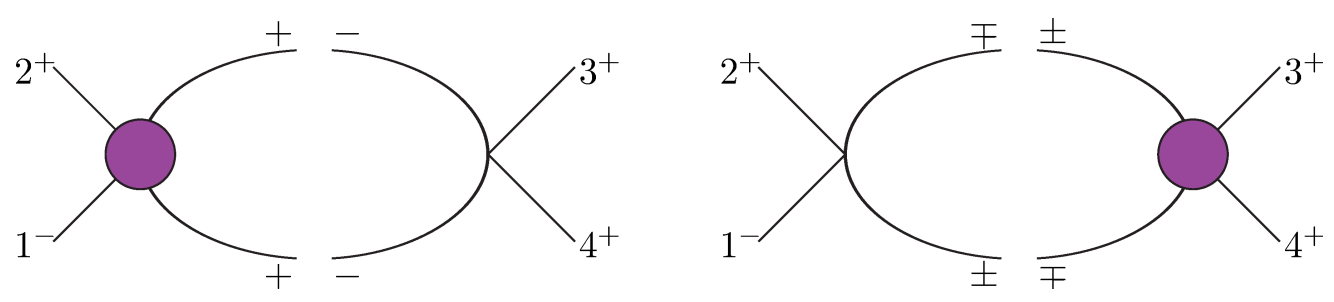

Figure 6.2: The bubbles for single minus

where we have used

$$
2 s I_{3}^{1 m}(s)+2 t I_{3}^{1 m}(t)=I_{4} .
$$

Note that there are no box functions in this amplitude and the triangle functions generate the soft part of the IR term. The overall coefficient of $\epsilon^{-1}$ is

$4 \frac{\alpha}{(4 \pi)^{2}}((t-u)+(s-u)) K_{++++}=-\frac{12}{(4 \pi)^{2}} u \alpha K_{++++}=-\frac{6}{(4 \pi)^{2}} \times A_{4}^{(0), \alpha}\left(1^{+}, 2^{+}, 3^{+}, 4^{+}\right)$,

which is proportional to the tree and so the one-loop UV infinity leads to a renormalisation of the $\alpha F^{3}$ term.

We also compute the one-loop contribution to the single-minus amplitude $A_{4}^{(1), \alpha}\left(1^{-}, 2^{+}, 3^{+}, 4^{+}\right)$. There are three non-zero configurations as shown in fig. 6.2. The first of these is

$$
\begin{aligned}
C_{2}^{A} & =A_{4}^{(0), \alpha}\left(1^{-}, 2^{+}, l_{2}^{+},-l_{1}^{+}\right) \times A_{4}^{(0), \alpha^{0}}\left(l_{1}^{-},-l_{2}^{-}, 3^{+}, 4^{+}\right) \\
& =-\alpha \frac{\left[2-l_{1}\right]^{2} s_{12} s_{2 l_{2}}}{[12]\left\langle 2 l_{2}\right\rangle\left\langle l_{2}-l_{1}\right\rangle\left[-l_{1} 1\right]} \times \frac{\left\langle l_{1}-l_{2}\right\rangle^{3}}{\left\langle-l_{2} 3\right\rangle\langle 34\rangle\left\langle 4 l_{1}\right\rangle} \\
& =-\alpha \frac{\langle 12\rangle}{\langle 34\rangle} \frac{\left[2 l_{1}\right]^{2}\left[2 l_{2}\right]}{\left[l_{1} 1\right]} \times \frac{\left\langle l_{1} l_{2}\right\rangle^{2}}{\left\langle l_{2} 3\right\rangle\left\langle 4 l_{1}\right\rangle} .
\end{aligned}
$$

This can be rearranged to

$$
C_{2}^{A}=-\alpha \frac{s_{12}^{2}}{\langle 34\rangle^{2}} \times \frac{\left[2 l_{1}\right]\left[2 l_{2}\right]\left\langle l_{1} 1\right\rangle\left\langle l_{2} 1\right\rangle}{\left(l_{1}-k_{1}\right)^{2}\left(l_{1}+k_{4}\right)^{2}},
$$

which is the two particle cut of a quadratic box. Replacing this by a covariant 


\section{LOOP AMPLITUDES IN AN EXTENDED THEORY OF GRAVITY}

integral and only keeping terms with a $s$-channel cut we obtain

$$
C_{2}^{A}=-\alpha K_{-+++}\left(\frac{s^{3} t}{2 u} I_{4}^{0 m}+\frac{s^{3}}{u} I_{3}^{1 m}(s)-s u I_{3}^{1 m}(s)-(2 t+s) I_{2}^{2 m}(s)\right)
$$

The other terms are a little more complex (requiring a quartic box integral) and yield

$$
\begin{aligned}
& C_{2}^{B}=-\alpha K_{-+++}\left(\frac{s t^{3}}{2 u} I_{4}^{0 m}(s, t)+\frac{s t^{2}}{u} I_{3}^{1 m}(s)-t I_{2}^{2 m}(s)\right), \\
& C_{2}^{C}=-\alpha K_{-+++}\left(\frac{s t u}{2} I_{4}^{0 m}(s, t)+u I_{2}^{2 m}(s)\right) .
\end{aligned}
$$

Combining these gives

$$
\begin{aligned}
A_{4}^{(1), \alpha}\left(1^{-}, 2^{+}, 3^{+}, 4^{+}\right)=-\alpha K_{-+++} & \left(\frac{s t}{2 u}\left(s^{2}+t^{2}+u^{2}\right) I_{4}^{0 m}(s, t)+\frac{\left(s^{2}+t^{2}-u^{2}\right)}{u}\left(s I_{3}^{1 m}(s)+t I_{3}^{1 m}(t)\right)\right. \\
& \left.\left.-(2 s+4 t) I_{2}^{2 m}(s)-(4 s+2 t) I_{2}^{2 m}(t)\right)\right)+R_{4} .
\end{aligned}
$$

The box functions combine with the triangle functions to generate the IR singular terms and the finite transcendental function,

$$
a I_{4}^{0 m}(s, t)+\sum_{j=s, t} b_{j} I_{3}^{1 m}(j)=A_{4}^{(0), \alpha^{0}} I_{4}+F_{4}
$$

where

$$
F_{4}=-\frac{\alpha}{(4 \pi)^{2}} K_{-+++} \times \frac{\left(s^{2}+u^{2}+t^{2}\right)}{u} \ln ^{2}(s / t),
$$

and we find no corrections to the IR structure.

We also find the UV terms, the coefficient of $\epsilon^{-1}$ as

$$
\frac{\alpha}{(4 \pi)^{2}} K_{-+++}((2 s+4 t)+(4 s+2 t))=-6 \frac{\alpha}{(4 \pi)^{2}} u K_{-+++}=-\frac{6}{(4 \pi)^{2}} A_{4}^{(0), \alpha}\left(1^{-}, 2^{+}, 3^{+}, 4^{+}\right),
$$

which matches the singularity found for the $A_{4}^{(1), \alpha}\left(1^{+}, 2^{+}, 3^{+}, 4^{+}\right)$case.

Equations (6.27) and (6.35) are the singularities in the bare amplitudes. The 
amplitudes contain universal collinear IR singularities [32]:

$$
-\frac{n \beta_{0} g^{2}}{2(4 \pi)^{2}} A_{n}^{(0), \alpha}=-\frac{22 N_{c} g^{2}}{3(4 \pi)^{2}} A_{n}^{(0), \alpha}
$$

where $\beta_{0}=11 N_{c} / 3$. We determine the UV divergence by first subtracting these from the bare singularity.

When renormalising the theory there must be a simultaneous renormalisation of $g^{2}$ and $\alpha$. The renormalisation of $g^{2}$ is unaltered since it is determined by the $\alpha^{0}$ amplitudes.

$$
g^{2} \alpha \longrightarrow g^{2} \alpha-g^{4} \beta_{0} \alpha+g^{2} \delta \alpha,
$$

so that (reinserting $g$ and $N_{c}$ )

$$
g^{2} \delta \alpha-\frac{g^{4} \beta_{0} \alpha}{\epsilon}-\frac{6 N_{c} \alpha}{(4 \pi)^{2} \epsilon}=-\frac{22 N_{c} g^{2}}{3(4 \pi)^{2}} \alpha
$$

so that

$$
\delta \alpha=\frac{g^{2} N_{c} \alpha}{(4 \pi)^{2} \epsilon}\left(6-\frac{11}{3}\right)=\frac{7 N_{c} \alpha}{3} \frac{g^{2}}{(4 \pi)^{2}} .
$$

This value of $7 N_{c} / 3$ matches previous calculations of the anomalous dimension of Yang-Mills extended by the $F^{3}$ operator $[180-183]$.

\subsection{Extended Gravity Amplitudes}

We now consider extending gravity by additional three point vertices. For gravitons with $h= \pm 2$ the possible three point amplitudes are 1

$$
\begin{aligned}
M_{3}^{(0), \alpha^{0}}\left(1^{-}, 2^{-}, 3^{+}\right) & =\frac{\langle 12\rangle^{6}}{\langle 23\rangle^{2}\langle 31\rangle^{2}}, \\
M_{3}^{(0), \alpha^{0}}\left(1^{+}, 2^{+}, 3^{-}\right) & =\frac{[12]^{6}}{[23]^{2}[31]^{2}}, \\
M_{3}^{(0), \alpha}\left(1^{+}, 2^{+}, 3^{+}\right) & =\alpha[12]^{2}[23]^{2}[31]^{2}, \\
M_{3}^{(0), \alpha}\left(1^{-}, 2^{-}, 3^{-}\right) & =\alpha\langle 12\rangle^{2}\langle 23\rangle^{2}\langle 31\rangle^{2} .
\end{aligned}
$$

\footnotetext{
${ }^{1}$ Where we use the conventions of $(2.19$.
} 


\section{LOOP AMPLITUDES IN AN EXTENDED THEORY OF GRAVITY}

From a Lagrangian viewpoint we are considering the theory

$$
L=\int d^{D} x \sqrt{-g}\left(R+\frac{\alpha}{60} R_{a b c d} R^{c d e f} R_{e f}^{a b}\right)
$$

The non-vanishing four-point $\mathcal{O}\left(\alpha^{0}\right)$ tree is

$$
M_{4: \text { tree }}^{\alpha^{0}}\left(1^{-}, 2^{-}, 3^{+}, 4^{+}\right)=-\frac{[34]\langle 12\rangle^{6}}{\langle 34\rangle\langle 13\rangle\langle 14\rangle\langle 23\rangle\langle 24\rangle},
$$

and the non-zero $\mathcal{O}(\alpha)$ trees are

$$
\begin{aligned}
M_{4}^{(0), \alpha}\left(1^{+}, 2^{+}, 3^{+}, 4^{+}\right) & =-10\left(\frac{s t}{\langle 12\rangle\langle 23\rangle\langle 34\rangle\langle 41\rangle}\right)^{2} s t u \\
& =-10 K_{++++}^{2} \times s t u, \\
M_{4}^{(0), \alpha}\left(1^{-}, 2^{+}, 3^{+}, 4^{+}\right) & =\left(\frac{[24]^{2}}{[12]\langle 23\rangle\langle 34\rangle[41]}\right)^{2} \frac{-s^{3} t^{3}}{u} \\
& =-K_{-+++}^{2} \times s t u .
\end{aligned}
$$

These four-point amplitudes due to a $R^{3}$ term have been computed using field theory methods [168] but can also be obtained from the three-point amplitudes by factorisation as shown in the previous chapter [112, 135]. These expressions also appear as the UV infinite pieces of both two-loop gravity in four dimensions [164, 171] and one-loop gravity in six dimensions [172].

We evaluate the one-loop amplitude for the all-plus amplitude via the two particle cuts

$$
C_{2}=\int d \operatorname{LIPS} M_{4}^{(0), \alpha}\left(1^{+}, 2^{+}, \ell_{2}^{+},-\ell_{1}^{+}\right) \times M_{4}^{(0), \alpha^{0}}\left(\ell_{1}^{-},-\ell_{2}^{-}, 3^{+}, 4^{+}\right) .
$$


Arranging the trees carefully we obtain

$$
\begin{aligned}
C_{2} & =10 \int d \operatorname{LIPS} \frac{[12]^{2}\left[\ell_{1} \ell_{2}\right]}{\left\langle\ell_{1} \ell_{2}\right\rangle}\left[2 \ell_{1}\right]\left[1 \ell_{2}\right]\left[2 \ell_{2}\right]\left[\ell_{1} 1\right] \times \frac{s\left\langle\ell_{1} \ell_{2}\right\rangle^{4}[34]}{\langle 34\rangle^{3}}\left(\frac{1}{s_{\ell_{1} 4}}+\frac{1}{s_{\ell_{1} 3}}\right) \\
& =10 \int d \operatorname{LIPS} \frac{s^{2}[12]^{2}[34]}{\langle 34\rangle^{3}}\left[2 \ell_{1}\right]\left[1 \ell_{2}\right]\left[2 \ell_{2}\right]\left[\ell_{1} 1\right] \times\left\langle\ell_{1} \ell_{2}\right\rangle^{2}\left(\frac{1}{s_{\ell_{1} 4}}+\frac{1}{s_{\ell_{1} 3}}\right) \\
& =10 \int d \operatorname{LIPS} \frac{s^{2}[12]^{4}[43]}{\langle 34\rangle^{3}}\left[1\left|\ell_{1}\right| 1\right\rangle\left[1\left|\ell_{2}\right| 1\right\rangle \times\left(\frac{1}{s_{\ell_{1} 4}}+\frac{1}{s_{\ell_{1} 3}}\right),
\end{aligned}
$$

which is the cut of a pair of quadratic triangle integrals. Replacing this by the covariant integral and evaluating yields

$$
10 \frac{t u}{s^{3}}[12]^{4}[34]^{4} \times s^{2} I_{3}^{1 m}(s)+10 \frac{[12]^{4}[34]^{4}}{s^{2}} \times\left(t^{2}+u^{2}-4 t u\right) I_{2}^{2 m}(s),
$$

where both integrals yield the same resultant integral function. After doubling this to account for inserting the $R^{3}$ operator on the opposite side of the cut, this can be rewritten as

$$
20 K_{++++}^{2} s t u \times s^{2} I_{3}^{1 m}(s)+20 K_{++++}^{2} \times s^{2}\left(t^{2}+u^{2}-4 t u\right) I_{2}^{2 m}(s) .
$$

The triangle functions correctly generate the IR terms and so the the amplitude can be expressed as

$$
M_{4}^{(1), \alpha}=M_{4}^{(0), \alpha} I_{4}+F_{4}+G_{4}+R_{4},
$$

with $F_{4}=0$ and

$$
\begin{gathered}
G_{4}=20 K_{++++}^{2}\left[s^{2}\left(t^{2}+u^{2}-4 t u\right) I_{2}^{2 m}(s)+t^{2}\left(s^{2}+u^{2}-4 s u\right) I_{2}^{2 m}(t)\right. \\
\left.+u^{2}\left(t^{2}+s^{2}-4 t s\right) I_{2}^{2 m}(u)\right] .
\end{gathered}
$$




\section{LOOP AMPLITUDES IN AN EXTENDED THEORY OF GRAVITY}

The coefficient of $\epsilon^{-1}$ is

$20 K_{++++}^{2} \times\left(s^{2}\left(t^{2}+u^{2}-4 t u\right)+\{s \leftrightarrow t\}+\{s \leftrightarrow u\}\right)=10 K_{++++}^{2} \times\left(s^{2}+t^{2}+u^{2}\right)^{2}$

In EH gravity the IR singularity is of the form $\sum s \ln (s) / \epsilon$ [117, 184] and the additional vertex will not affect this since the vertex has a higher momentum power count. Therefore the rational $\epsilon^{-1}$ singularities in eq. (6.50) represents the ultraviolet divergence. The Yang-Mills case, whose UV singularity is proportional to the $\mathcal{O}(\alpha)$ tree-level Yang-Mills amplitude (6.35), differs in form compared to the UV singularity in the gravitational case - which is not proportional to the tree-level $\mathcal{O}(\alpha)$ gravity amplitude $(6.50)$. The implication of this being that the two theories are renormalised in differing ways. The extended Yang-Mills theory is renormalised via a redefinition of the cubic vertex, whereas the extended gravity theory but must be renormalised by the addition of a four-point vertex produced by a higher-dimension local operator.

As a consistency check we also consider the single-minus amplitude. The $s$-channel bubble in this case has three configurations:

$$
\begin{array}{rll}
M_{4}^{(0), \alpha}\left(1^{-}, 2^{+}, \ell_{2}^{+},-\ell_{1}^{+}\right) & \times & M_{4}^{(0), \alpha^{0}}\left(\ell_{1}^{-},-\ell_{2}^{-}, 3^{+}, 4^{+}\right), \\
M_{4}^{(0), \alpha^{0}}\left(1^{-}, 2^{+}, \ell_{2}^{\mp},-\ell_{1}^{ \pm}\right) & \times & M_{4}^{(0), \alpha}\left(\ell_{1}^{ \pm},-\ell_{2}^{\mp}, 3^{+}, 4^{+}\right) .
\end{array}
$$

These give contributions to the coefficients of the bubble integral functions. We find

$$
\begin{aligned}
& c_{A}=K_{-+++}^{2} \times \frac{s^{2}}{t u}\left(2\left(t^{4}+u^{4}\right)+5 u t\left(t^{2}+u^{2}\right)\right), \\
& c_{B}=K_{-+++}^{2} \times \frac{s^{2}}{t u}\left(2\left(t^{4}+u^{4}\right)-3 u t\left(t^{2}+u^{2}\right)\right),
\end{aligned}
$$

giving the overall coefficient of $I_{2}^{2 m}(s)$ to be

$$
c=K_{-+++}^{2} \times \frac{s^{2}}{t u}\left(4\left(t^{4}+u^{4}\right)+2 u t\left(t^{2}+u^{2}\right)\right) .
$$


Extracting the UV divergence we find

$$
\begin{aligned}
& \frac{1}{\epsilon} \times\left(\frac{s^{2}}{t u}\left(4\left(t^{4}+u^{4}\right)+2 u t\left(t^{2}+u^{2}\right)\right)+\{s \leftrightarrow u\}+\{s \leftrightarrow t\}\right) \\
& =0
\end{aligned}
$$

and so this amplitude has no UV divergence.

In summary, the UV infinities for the four-point one-loop amplitudes are, (re-inserting the appropriate factors)

$$
\begin{aligned}
& \left.M_{4: 1-\text { loop }}^{\alpha}\left(1^{-}, 2^{-}, 3^{+}, 4^{+}\right)\right|_{1 / \epsilon}=0 \\
& \left.M_{4: 1-\text { loop }}^{\alpha}\left(1^{+}, 2^{+}, 3^{+}, 4^{+}\right)\right|_{1 / \epsilon}=\alpha \frac{i}{(4 \pi)^{2}}\left(\frac{\kappa}{2}\right)^{4} \frac{1}{\epsilon} \times 10 K_{++++}^{2} \times\left(s^{2}+t^{2}+u^{2}\right)^{2} \\
& \left.M_{4: 1-\text { loop }}^{\alpha}\left(1^{-}, 2^{+}, 3^{+}, 4^{+}\right)\right|_{1 / \epsilon}=0
\end{aligned}
$$

Unlike the Yang-Mills case, the UV infinity is not removed by a renormalisation of the three-point vertex but requires the addition of a four-point vertex which acts as a counterterm. The counterterm is examined in [113] and is given by,

$$
R_{\text {counter }}^{4}=\frac{i}{(4 \pi)^{4} \epsilon} R_{c d}^{a b} R^{c d} R_{g h}^{e f} R_{a b}^{g h}
$$

\subsection{Beyond Cubic Vertices}

The non-extended theory of graviton scattering ( and of gluons) is constructible: that is the entire tree-level $S$-matrix can be generated by demanding that the amplitudes are factorisable 163 . In practice the factorisation can be excited by the BCFW shift. In the extended theory the leading deformation of the treelevel $S$-matrix is also constructible [112] albeit by using alternative shifts [37, 162. However at order $\alpha^{2}$, if we consider $M_{4: \text { tree }}^{\alpha^{2}}\left(1^{-}, 2^{-}, 3^{+}, 4^{+}\right)$there is a single factorisation as shown in fig. 6.3. The amplitude

$$
M_{4: \text { tree }}^{\alpha^{2}}\left(1^{-}, 2^{-}, 3^{+}, 4^{+}\right)=\alpha^{2}\langle 12\rangle^{4}[34]^{4}\left(\frac{t u+\beta s^{2}}{s}\right),
$$




\section{LOOP AMPLITUDES IN AN EXTENDED THEORY OF GRAVITY}

has the correct factorisation for any choice of $\beta$. This ambiguity means we also have to specify the four-point amplitude to determine the $S$-matrix. In the diagrammar approach this ambiguity arises due to the existence of a polynomial function with the correct symmetries and spinor and momentum weight. From a field theory perspective, additional counterterms can contribute to this amplitude. Specifically, we could deform the theory via

$$
R \longrightarrow R+C_{\alpha} R^{3}+C_{\beta} D^{2} R^{4}
$$

and the four-point amplitude is only specified once $C_{\alpha}$ and $C_{\beta}$ are determined.

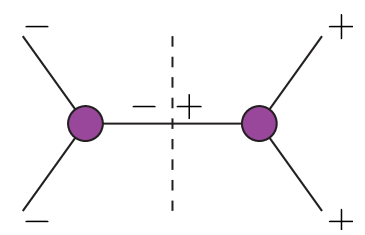

Figure 6.3: Factorisations of the four-point MHV amplitude at $\alpha^{2}$.

From a constructibility viewpoint, defining the theory from its four-point amplitudes is much less constrained than using the three-point amplitudes because momenta constraints do not limit the vertex to be constructed only from $\lambda_{i}$ or $\bar{\lambda}_{i}$ but can involve both (or more likely momenta invariants). Specifically we could introduce a fundamental amplitude

$$
M_{4: \text { tree }}\left(1^{-}, 2^{-}, 3^{+}, 4^{+}\right)=\beta^{\prime} s\langle 12\rangle^{4}[34]^{4}
$$

From a Lagrangian perspective this would be implemented by a $D^{2} R^{4}$ operator giving non-vanishing $M\left(1^{-}, 2^{-}, 3^{+}, 4^{+}\right)$but vanishing $M\left(1^{+}, 2^{+}, 3^{+}, 4^{+}\right)$and $M\left(1^{-}, 2^{+}, 3^{+}, 4^{+}\right)$. As the all-plus and single-minus amplitudes vanish in a supersymmetric theory, any operator compatible with supersymmetry that generates a non-vanishing four-point MHV amplitude will suffice.

Although constructibility from three-point vertices is an attractive concept, unfortunately we find the theory is not completely specified by the three-point vertex. 


\subsection{Conclusion}

We have studied the $S$-matrix of extended Yang-Mills and gravity using a diagrammar approach in which the theory is defined by its on-shell amplitudes. If we wish to extend either pure Yang-Mills or gravity by the addition of a threepoint interaction there is a unique choice governed by the unique choice of $F^{3}$ or $R^{3}$ operator from the field theory perspective. This choice leads to a theory in which the leading deformation is constructible from three-point amplitudes although higher order deformations require further information to fix the amplitudes. In this chapter, we have studied the one-loop corrections to these theories and demonstrated how unitarity can be used to examine the UV structure of the theories to understand the renormalisability of the theory. For Yang-Mills the one-loop UV infinities are renormalised by the four-point vertex at leading order. For gravity however the UV infinities must be cancelled by four-point amplitudes arising from a different source. Extending the $S$-matrix of gravity by the addition of the minimal three-point amplitude is equivalent to adding $R^{3}$ terms to the Lagrangian. From a Lagrangian view point this is then renormalised at one-loop by $R^{4}$ counterterms. 
6. LOOP AMPLITUDES IN AN EXTENDED THEORY OF GRAVITY 


\section{Chapter 7}

\section{Conclusions}

This thesis has reviewed techniques and recent discoveries made in pure YangMills and extended theories of gravity by the use of four-dimensional unitarity and recursive techniques. Splitting the calculation of an amplitude into two parts allows for a vast simplification in the deduction of the branch cut singularity structures, while leaving the rational terms to be determined. Augmented recursion has proven to be an effective method of capturing the full rational parts of various loop amplitudes which in general contain double poles. While currently this constitutes the majority of the calculation at higher multiplicities, it still presents the most promising way to determine rational terms of amplitudes that contain higher order poles. We also used these techniques to examine a theory of extended gravity that provides the counterterms for two-loop gravity in four dimensions.

Through the investigations into the scattering of gluons, we continue to add to the dictionary of amplitudes required to describe partonic cross sections relevant in collider experiments. Having access to higher multiplicity expressions allows IR safe cross sections to be expressed at higher orders in the coupling constant and furthermore, promotes progress in the theoretical understanding of the structure

of gluon amplitudes. In chapter 3 we have shown that the applicability and effectiveness of these methods have range even at the two-loop level in pure YangMills. Moreover, given that these techniques have computed the first six- and seven-gluon expressions for the leading in colour two-loop all-plus amplitude, 


\section{CONCLUSIONS}

they offer a more promising path to discovering all- $n$ formulae for amplitudes than other conventional techniques.

In chapter 4 we demonstrated the efficacy of four-dimensional techniques for amplitudes at all orders in the number of colours. Examining the two-loop fivepoint all-plus amplitude, we showed the simple extension to determining full colour amplitudes comes from promoting the leading in colour partial amplitudes with their full colour counterparts when computing the amplitude using unitarity cuts and augmented recursion. The cut constructible parts of the amplitude follow straightforwardly from the leading in colour case. The rational part of the amplitude follows suit, however we showed that the double pole contributions, to all orders in $N_{c}$, can be derived from the leading in colour doubly off-shell adjacent and non-adjacent currents. This result has its foundation in the decoupling identities for the one-loop current and shows, at least at two-loop, that we can reuse the leading in colour currents to generate the (sub-)subleading in colour rational terms at two-loop. This is a promising discovery and has already proven fruitful, with the expression for the two-loop six-point all-plus amplitude given shortly after the five-point. Furthermore, the simple expression for the sub-subleading single trace partial amplitude found by these methods has led to an all- $n$ form. Providing a direct method of calculating a full colour amplitude at two-loops for a particular helicity amplitude is a demonstration of the staying power that four-dimensional unitarity still possesses to this day.

The recursive methods we employ also provide a simple way of examining a theory of extended gravity. In chapter 5, we showed that we could generate the tree-level $S$-matrix of the leading deformation of Einstein-Hilbert gravity extended by an $\alpha R^{3}$ term. This leading $\mathcal{O}(\alpha)$ deformation is determined by fixing the three-point amplitudes and demanding factorisation. In order to determine the theory to higher orders in $\alpha$, more constraints are required. We then considered the renormalisability of this theory in chapter 6 , by extracting the UV infinite terms, and identifying the counterterm required to renormalise the theory. We also compared this with the tree-level and one-loop amplitudes of a related theory, pure Yang-Mills extended by an $F^{3}$ term, whose double copy gives a gravitational theory extended by $\alpha R^{3}+\sqrt{\alpha} R^{2} \phi$. We showed that the pure Yang-Mills 
theory was renormalised by a redefinition of the coupling constant.

The next step for calculations involving four-dimensional unitarity would be to calculate the single-minus two-loop amplitude. The IR singular structure of this amplitude remains the same, since the associated tree-level amplitudes vanishes. However, the simplifications we were able to use to study the all-plus do not apply in general here. In this case, we must consider genuine two-loop integrals when computing the cuts of the amplitude. In terms of the rational part of this amplitude, it would be preferable to reach a general prescription for these terms at two-loops, but in its absence we must consider the inclusion of an MHV current. Since the current is still a one-loop object, we expect that the decoupling identities will still provide a route to determining the (sub)-subleading in colour rational terms.

The results and evolving methods presented in this thesis continue to prove the relevance of four-dimensional unitarity methods. Its ability to determine physically relevant scattering amplitudes has seen success and motivates further efforts along this vein. 
7. CONCLUSIONS 


\section{Appendix A}

\section{Feynman Rules for Gluon Amplitudes}

Here we briefly summarise the Feynman rules for gluon amplitudes, where we present the forms with the factors of $i$ reinstated. In the lightcone axial gauge, the gluon propagator is,

$$
\frac{i}{p^{2}} d_{\mu \nu}=\frac{i}{p^{2}}\left(-\eta_{\mu \nu}+2 \frac{p_{\mu} q_{\nu}+q_{\mu} p_{\nu}}{2 q \cdot p}\right)
$$

The Feynman rules for three- and four-gluon vertices derived from the Yang-Mills Lagrangian, with colour are given as,

$$
V_{3}\left(p_{1}^{\mu}, p_{2}^{\nu}, p_{3}^{\rho}\right)=i g f^{a b c}\left[\eta^{\mu \nu}\left(p_{1}-p_{2}\right)^{\rho}+\eta^{\nu \rho}\left(p_{2}-p_{3}\right)^{\mu}+\eta^{\rho \mu}\left(p_{3}-p_{1}\right)^{\nu}\right]
$$

$V_{4}\left(p_{1}^{\mu}, p_{2}^{\nu}, p_{3}^{\rho}, p_{3}^{\sigma}\right)=i g^{2}\left[f^{a b e} f^{c d e}\left(\eta_{\mu \rho} \eta_{\nu \sigma}-\eta_{\mu \sigma} \eta_{\nu \rho}\right)+f^{a c e} f^{d b e}\left(\eta_{\mu \sigma} \eta_{\rho \nu}-\eta_{\mu \nu} \eta_{\rho \sigma}\right)+f^{a d e} f^{b c e}\left(\eta_{\mu \nu} \eta_{\sigma \rho}-\eta_{\mu \rho} \eta_{\sigma \nu}\right)\right]$ 


\section{A. FEYNMAN RULES FOR GLUON AMPLITUDES}

After colour decomposition, the colour ordered Feynman Rules for the three- and four-gluon vertices are given as,

$$
\begin{aligned}
&\left.V_{3}\left(p_{1}^{\mu}, p_{2}^{\nu}, p_{3}^{\rho}\right)\right|_{c . o .}=i\left[\eta^{\mu \nu}\left(p_{1}-p_{2}\right)^{\rho}+\eta^{\nu \rho}\left(p_{2}-p_{3}\right)^{\mu}+\eta^{\rho \mu}\left(p_{3}-p_{1}\right)^{\nu}\right] \\
&\left.V_{4}\left(p_{1}^{\mu}, p_{2}^{\nu}, p_{3}^{\rho}, p_{3}^{\sigma}\right)\right|_{\text {c.o. }}=i\left[2 \eta^{\mu \rho} \eta^{\nu \sigma}-\eta^{\mu \nu} \eta^{\rho \sigma}-\eta^{\mu \sigma} \eta^{\nu \rho}\right]
\end{aligned}
$$

Gluons are massless spin 1 gauge bosons and have two polarisations, $\epsilon_{\mu}^{+}$and $\epsilon_{\mu}^{-}$. To compute a gluon amplitude the Feynman rules for the vertex interactions must be contracted with the specific gluon polarisation corresponding to the relevant gluon. In the spinor helicity formalism, the polarisation vectors are given as,

$$
\epsilon_{\mu}^{-}(p, q)=\frac{\left[q\left|\gamma_{\mu}\right| p\right\rangle}{\sqrt{2}[p q]}, \quad \epsilon_{\mu}^{+}(p, q)=\frac{\left[p\left|\gamma_{\mu}\right| q\right\rangle}{\sqrt{2}\langle q p\rangle}
$$




\section{Appendix B}

\section{Integrals}

This section lists the scalar one-loop integrals relevant for calculating Yang-Mills and gravity one-loop amplitudes and the two-loop all-plus amplitude [53, 55, 188, 189].

\section{B.1 Integral Functions}

This section lists the scalar one-loop integrals relevant for calculating Yang-Mills and gravity one-loop amplitudes and the two-loop all-plus amplitude [53, 55, 188, 189].

\section{B.1.1 Bubble Integrals}

Massless or one-mass bubbles vanish since tadpoles vanish in dimensional regularisation. The only non-vanishing bubble integrals are the UV divergent massive bubble integrals,

$$
I_{2}^{2 m}(S)=\left(\frac{\mu^{2}}{-S}\right)^{\epsilon}\left[\frac{1}{\epsilon}+2\right]+\mathcal{O}(\epsilon) .
$$

\section{B.1.2 Triangle Integrals}

The IR divergent massless, one- and two-mass triangles are given below, along with the finite three-mass triangle. 


\section{B. INTEGRALS}

The 1-mass triangle is,

$$
\begin{aligned}
I_{3}^{1 m}\left(K_{1}^{2}\right) & =\frac{\mu^{2 \epsilon}}{\epsilon^{2}}\left[\frac{\left(-K_{1}^{2}\right)^{-\epsilon}}{K_{1}^{2}}\right] \\
& =\frac{1}{K_{1}^{2}}\left[\frac{1}{\epsilon^{2}}-\frac{1}{\epsilon} \ln \left(\frac{-K_{1}^{2}}{\mu^{2}}\right)+\frac{1}{2} \ln ^{2}\left(\frac{-K_{1}^{2}}{\mu^{2}}\right)\right]+\mathcal{O}(\epsilon) .
\end{aligned}
$$

The two-mass triangle is,

$$
\begin{aligned}
I_{3}^{2 m}\left(K_{1}^{2}, K_{2}^{2}\right) & =\frac{\mu^{2 \epsilon}}{\epsilon^{2}}\left[\frac{\left(-K_{1}^{2}\right)^{-\epsilon}-\left(-K_{2}^{2}\right)^{-\epsilon}}{K_{1}^{2}-K_{2}^{2}}\right] \\
& =\frac{1}{K_{1}^{2}-K_{2}^{2}}\left[\frac{1}{\epsilon} \ln \left(\frac{K_{2}^{2}}{K_{1}^{2}}\right)+\frac{1}{2}\left(\ln ^{2}\left(\frac{-K_{1}^{2}}{\mu^{2}}\right)-\ln ^{2}\left(\frac{-K_{2}^{2}}{\mu^{2}}\right)\right)\right]+\mathcal{O}(\epsilon) .
\end{aligned}
$$

The three-mass triangle [55, 188] is given as,

$$
I_{3}^{3 m}\left(S, T, K_{1}^{2}, K_{2}^{2}, K_{3}^{2}\right)=\frac{1}{\sqrt{\Delta_{3}}} \sum_{j=1}^{3}\left[\operatorname{Li}_{2}\left(-\frac{1+i \delta_{j}}{1-i \delta_{j}}\right)-\mathrm{Li}_{2}\left(-\frac{1-i \delta_{j}}{1+i \delta_{j}}\right)\right]+\mathcal{O}(\epsilon)
$$

where

$$
\begin{gathered}
\delta_{1}=\frac{K_{1}^{2}-K_{2}^{2}-K_{3}^{2}}{\sqrt{\Delta_{3}}}, \\
\delta_{1}=\frac{-K_{1}^{2}+K_{2}^{2}-K_{3}^{2}}{\sqrt{\Delta_{3}}}, \\
\delta_{1}=\frac{-K_{1}^{2}-K_{2}^{2}+K_{3}^{2}}{\sqrt{\Delta_{3}}},
\end{gathered}
$$

with

$$
\Delta_{3}=-\left[K_{1}^{2}+K_{2}^{2}+K_{3}^{2}\right]^{2} .
$$




\section{B.1 Integral Functions}

\section{B.1.3 Box Integrals}

The IR divergent massless, one-mass, two-mass and three-mass box integrals are given below along with the finite four-mass triangle.

The massless box is,

$$
I_{4}^{0 m}(S, T)=\frac{\mu^{2 \epsilon}}{S T}\left[\frac{2}{\epsilon^{2}}\left((-S)^{-\epsilon}+(-T)^{-\epsilon}\right)-\ln ^{2}\left(\frac{S}{T}\right)-\pi^{2}\right]+\mathcal{O}(\epsilon) .
$$

The 1-mass box is,

$$
\begin{aligned}
I_{4}^{1 m}\left(S, T, K_{1}^{2}\right)=\frac{\mu^{2 \epsilon}}{S T} & {\left[\frac{2}{\epsilon^{2}}\left((-S)^{-\epsilon}+(-T)^{-\epsilon}-\left(-K_{1}^{2}\right)^{-\epsilon}\right)\right.} \\
& \left.\quad-2 \operatorname{Li}_{2}\left(1-\frac{K_{1}^{2}}{S}\right)-2 \operatorname{Li}_{2}\left(1-\frac{K_{1}^{2}}{T}\right)-\ln ^{2}\left(\frac{S}{T}\right)-\frac{\pi^{2}}{3}\right]+\mathcal{O}(\epsilon) .
\end{aligned}
$$

Next we have two types of two-mass boxes. The two-mass hard box, where the massive legs are adjacent is,

$$
\begin{aligned}
& I_{4}^{2 m h}\left(S, T, K_{1}^{2}, K_{2}^{2}\right)=\frac{\mu^{2 \epsilon}}{S T}\left[\frac{2}{\epsilon^{2}}\left((-S)^{-\epsilon}+(-T)^{-\epsilon}-\left(-K_{1}^{2}\right)^{-\epsilon}-\left(-K_{2}^{2}\right)^{-\epsilon}\right)\right. \\
& \left.+\frac{1}{\epsilon^{2}} \frac{\left(-K_{1}^{2}\right)^{-\epsilon}\left(-K_{2}^{2}\right)^{-\epsilon}}{(-S)^{-\epsilon}}-2 \operatorname{Li}_{2}\left(1-\frac{K_{1}^{2}}{T}\right)-2 \operatorname{Li}_{2}\left(1-\frac{K_{2}^{2}}{T}\right)-\ln ^{2}\left(\frac{S}{T}\right)\right]+\mathcal{O}(\epsilon) .
\end{aligned}
$$

The two-mass easy box, where the massive legs are not adjacent is,

$$
\begin{aligned}
& I_{4}^{2 m e}\left(S, T, K_{1}^{2}, K_{3}^{2}\right)=\frac{\mu^{2 \epsilon}}{S T-K_{1}^{2} K_{3}^{2}} \\
& \times\left[\frac{2}{\epsilon^{2}}\left((-S)^{-\epsilon}+(-T)^{-\epsilon}-\left(-K_{1}^{2}\right)^{-\epsilon}-\left(-K_{3}^{2}\right)^{-\epsilon}\right)-2 \mathrm{Li}_{2}\left(1-\frac{K_{1}^{2}}{S}\right)-2 \mathrm{Li}_{2}\left(1-\frac{K_{1}^{2}}{T}\right)\right. \\
& \left.-2 \mathrm{Li}_{2}\left(1-\frac{K_{3}^{2}}{S}\right)-2 \mathrm{Li}_{2}\left(1-\frac{K_{3}^{2}}{T}\right)+2 \mathrm{Li}_{2}\left(1-\frac{K_{1}^{2} K_{3}^{2}}{S T}\right)-\ln ^{2}\left(\frac{S}{T}\right)-\frac{\pi^{2}}{3}\right]+\mathcal{O}(\epsilon) .
\end{aligned}
$$


The three-mass box is given as,

$$
\begin{aligned}
& I_{4}^{3 m}\left(S, T, K_{1}^{2}, K_{2}^{2}, K_{3}^{2}\right)=\frac{\mu^{2 \epsilon}}{S T-K_{1}^{2} K_{3}^{2}}\left[\frac{2}{\epsilon^{2}}\left((-S)^{-\epsilon}+(-T)^{-\epsilon}-\left(-K_{1}^{2}\right)^{-\epsilon}-\left(-K_{2}^{2}\right)^{-\epsilon}-\left(-K_{3}^{2}\right)^{-\epsilon}\right)\right. \\
& +\frac{1}{\epsilon^{2}} \frac{\left(-K_{1}^{2}\right)^{-\epsilon}\left(-K_{2}^{2}\right)^{-\epsilon}}{(-T)^{-\epsilon}}+\frac{1}{\epsilon^{2}} \frac{\left(-K_{2}^{2}\right)^{-\epsilon}\left(-K_{3}^{2}\right)^{-\epsilon}}{(-S)^{-\epsilon}}-2 \mathrm{Li}_{2}\left(1-\frac{K_{1}^{2}}{S}\right)-2 \operatorname{Li}_{2}\left(1-\frac{K_{3}^{2}}{T}\right) \\
& \left.+2 \operatorname{Li}_{2}\left(1-\frac{K_{1}^{2} K_{3}^{2}}{S T}\right)-\ln ^{2}\left(\frac{S}{T}\right)\right]+\mathcal{O}(\epsilon) .
\end{aligned}
$$

Finally, the finite four-mass box is given by,

$$
\begin{aligned}
& I_{4}^{4 m}\left(S, T, K_{1}^{2}, K_{2}^{2}, K_{3}^{2}, K_{4}^{2}\right)=\frac{-\mu^{2 \epsilon}}{K_{2}^{2} K_{3}^{2}}\left[\operatorname{Li}_{2}\left(\frac{1}{2}\left(1-r_{1}+r_{2}-\rho\right)\right)-\operatorname{Li}_{2}\left(\frac{1}{2}\left(1-r_{1}+r_{2}+\rho\right)\right)\right. \\
& \left.+\operatorname{Li}_{2}\left(-\frac{1}{2 r_{1}}\left(1-r_{1}-r_{2}+\rho\right)\right)-\operatorname{Li}_{2}\left(\frac{1}{2 r_{1}}\left(1-r_{1}-r_{2}-\rho\right)\right)-\frac{1}{2} \ln \left(-\frac{r_{1}}{r_{2}^{2}}\right) \ln \left(\frac{1+r_{1}-r_{2}+\rho}{1+r_{1}-r_{2}-\rho}\right)\right]
\end{aligned}
$$

where

$$
\rho=\sqrt{1-2 r_{1}-2 r_{2}+\left(r_{1}-r_{2}\right)^{2}},
$$

with

$$
r_{1}=\frac{K_{1}^{2} K_{3}^{2}}{S T}, \quad r_{2}=\frac{K_{2}^{2} K_{4}^{2}}{S T} .
$$




\section{Appendix C}

\section{Derivation of the One-Loop Six-Gluon Current}

The six-point single minus one-loop amplitude is [38]:

$$
\begin{aligned}
A_{6: 1}^{(1)} & \left(\alpha^{-}, \beta^{+}, c^{+}, d^{+}, e^{+}, f^{+}\right) \\
& =\frac{i}{3}\left[\frac{\left[f\left|K_{\beta c}\right| \alpha\right\rangle^{3}}{\langle\alpha \beta\rangle\langle\beta c\rangle\langle d e\rangle^{2} t_{\alpha \beta c}\left[f\left|K_{\alpha \beta}\right| c\right\rangle}+\frac{\left[\beta\left|K_{c d}\right| \alpha\right\rangle^{3}}{\langle c d\rangle^{2}\langle e f\rangle\langle f \alpha\rangle t_{\beta c d}\left[\beta\left|K_{c d}\right| e\right\rangle}\right. \\
& +\frac{[\beta f]^{3}}{[\alpha \beta][f \alpha] t_{c d e}}\left(\frac{[\beta c][c d]}{\langle d e\rangle\left[\beta\left|K_{c d}\right| e\right\rangle}-\frac{[d e][e f]}{\langle c d\rangle\left[f\left|K_{\alpha \beta}\right| c\right\rangle}+\frac{[c e]}{\langle c d\rangle\langle d e\rangle}\right) \\
& -\frac{\langle\alpha c\rangle^{3}[\beta c]\langle\beta d\rangle}{\langle\beta c\rangle^{2}\langle c d\rangle^{2}\langle d e\rangle\langle e f\rangle\langle f \alpha\rangle}+\frac{\langle\alpha e\rangle^{3}[e f]\langle d f\rangle}{\langle\alpha \beta\rangle\langle\beta c\rangle\langle c d\rangle\langle d e\rangle^{2}\langle e f\rangle^{2}} \\
& \left.-\frac{\langle\alpha d\rangle^{3}\langle c e\rangle\left[d\left|K_{\beta c}\right| \alpha\right\rangle}{\langle\alpha \beta\rangle\langle\beta c\rangle\langle c d\rangle^{2}\langle d e\rangle^{2}\langle e f\rangle\langle f \alpha\rangle}\right]
\end{aligned}
$$

We manipulate the amplitude into a form where taking the off-shell continuation exactly reproduces the factorisations shown in fig.2.8, thus satisfying condition I. As the starting point is the six-point amplitude, condition II is automatically encoded. First we collect terms with $\langle\alpha \beta\rangle$ and $[\alpha \beta]$ in the denominator 
as these contribute to the factorisations.

$$
\begin{aligned}
A_{6: 1}^{(1)}\left(\alpha^{-}, \beta^{+}, c^{+}, d^{+}, e^{+}, f^{+}\right) \\
=\frac{i}{3}\left[\frac{1}{\langle\alpha \beta\rangle\langle\beta c\rangle\langle d e\rangle^{2}}\left(\frac{\left[f\left|K_{\beta c}\right| \alpha\right\rangle^{3}}{t_{\alpha \beta c}\left[f\left|K_{\alpha \beta}\right| c\right\rangle}+\frac{\langle\alpha e\rangle^{3}[e f]\langle d f\rangle}{\langle c d\rangle\langle e f\rangle^{2}}-\frac{\langle\alpha d\rangle^{3}\langle c e\rangle\left[d\left|K_{\beta c}\right| \alpha\right\rangle}{\langle c d\rangle^{2}\langle e f\rangle\langle f \alpha\rangle}\right)\right. \\
\quad+\frac{[\beta f]^{3}}{[\alpha \beta][f \alpha] t_{c d e}}\left(\frac{[\beta c][c d]}{\langle d e\rangle\left[\beta\left|K_{c d}\right| e\right\rangle}-\frac{[d e][e f]}{\langle c d\rangle\left[f\left|K_{\alpha \beta}\right| c\right\rangle}+\frac{[c e]}{\langle c d\rangle\langle d e\rangle}\right) \\
\left.\quad+\frac{\left[\beta\left|K_{c d}\right| \alpha\right\rangle^{3}}{\langle c d\rangle^{2}\langle e f\rangle\langle f \alpha\rangle t_{\beta c d}\left[\beta\left|K_{c d}\right| e\right\rangle}-\frac{\langle\alpha c\rangle^{3}[\beta c]\langle\beta d\rangle}{\langle\beta c\rangle^{2}\langle c d\rangle^{2}\langle d e\rangle\langle e f\rangle\langle f \alpha\rangle}\right] .
\end{aligned}
$$

Expanding in $\langle\alpha \beta\rangle$ to isolate the singular pieces we find

$$
\begin{aligned}
A_{6: 1}^{(1)}\left(\alpha^{-}, \beta^{+}, c^{+}, d^{+}, e^{+}, f^{+}\right) \\
=\frac{i}{3}\left[\frac{1}{\langle\alpha \beta\rangle\langle\beta c\rangle\langle d e\rangle^{2}}\left(\frac{[f c]^{3}\langle c \alpha\rangle^{3}}{t_{\alpha \beta c}\left[f\left|K_{\alpha \beta}\right| c\right\rangle}+\frac{\langle\alpha e\rangle^{3}[e f]\langle d f\rangle}{\langle c d\rangle\langle e f\rangle^{2}}-\frac{\langle\alpha d\rangle^{3}\langle c e\rangle[d c]\langle c \alpha\rangle}{\langle c d\rangle^{2}\langle e f\rangle\langle f \alpha\rangle}\right)\right. \\
\quad+\frac{1}{\langle\beta c\rangle\langle d e\rangle^{2}}\left(\frac{[\beta f]\left(3[f|c| \alpha\rangle^{2}+3[f|c| \alpha\rangle[f|\beta| \alpha\rangle+[f|\beta| \alpha\rangle^{2}\right)}{t_{\alpha \beta c}\left[f\left|K_{\alpha \beta}\right| c\right\rangle}-\frac{\langle\alpha d\rangle^{3}\langle c e\rangle[\beta d]}{\langle c d\rangle^{2}\langle e f\rangle\langle f \alpha\rangle}\right) \\
\quad+\frac{[\beta f]^{3}}{[\alpha \beta][f \alpha] t_{c d e}}\left(\frac{[\beta c][c d]}{\langle d e\rangle\left[\beta\left|K_{c d}\right| e\right\rangle}-\frac{[d e][e f]}{\langle c d\rangle\left[f\left|K_{\alpha \beta}\right| c\right\rangle}+\frac{[c e]}{\langle c d\rangle\langle d e\rangle}\right) \\
\left.\quad+\frac{\left[\beta\left|K_{c d}\right| \alpha\right\rangle^{3}}{\langle c d\rangle^{2}\langle e f\rangle\langle f \alpha\rangle t_{\beta c d}\left[\beta\left|K_{c d}\right| e\right\rangle}-\frac{\langle\alpha c\rangle^{3}[\beta c]\langle\beta d\rangle}{\langle\beta c\rangle^{2}\langle c d\rangle^{2}\langle d e\rangle\langle e f\rangle\langle f \alpha\rangle}\right] .
\end{aligned}
$$

The first line of eq. (C.3) has the $\langle\alpha \beta\rangle^{-1}$ factor. This can be rewritten using,

$$
\begin{aligned}
\frac{1}{\langle\alpha \beta\rangle\langle\beta c\rangle} & =\frac{[\beta \alpha]\left[q\left|K_{\alpha \beta}\right| c\right\rangle\langle\beta q\rangle}{s_{\alpha \beta}\langle\beta c\rangle\langle\beta q\rangle\left[q\left|K_{\alpha \beta}\right| c\right\rangle}=\frac{[\beta \alpha]\left(\left[q\left|K_{\alpha \beta}\right| q\right\rangle\langle\beta c\rangle+\left[q\left|K_{\alpha \beta}\right| \beta\right\rangle\langle c q\rangle\right)}{s_{\alpha \beta}\langle\beta c\rangle\langle\beta q\rangle\left[q\left|K_{\alpha \beta}\right| c\right\rangle} \\
& =\frac{1}{\langle\alpha q\rangle\langle\beta q\rangle^{2}}\left(\frac{\langle q|\alpha \beta| q\rangle\left[q\left|K_{\alpha \beta}\right| q\right\rangle}{s_{\alpha \beta}\left[q\left|K_{\alpha \beta}\right| c\right\rangle}+\frac{\langle q \beta\rangle\langle q c\rangle[q|\alpha| q\rangle}{\langle\beta c\rangle\left[q\left|K_{\alpha \beta}\right| c\right\rangle}\right)
\end{aligned}
$$

which is an algebraic identity for any $q$, but we specifically identify $q$ with the 
axial gauge reference momenta. Applying the formula to (C.3),

$$
\begin{aligned}
& A_{6: 1}^{(1)}\left(\alpha^{-}, \beta^{+}, c^{+}, d^{+}, e^{+}, f^{+}\right)= \\
& \frac{i}{3}\left[\frac{\langle q|\alpha \beta| q\rangle\left[q\left|K_{\alpha \beta}\right| q\right\rangle}{s_{\alpha \beta}\left[q\left|K_{\alpha \beta}\right| c\right\rangle\langle\alpha q\rangle\langle\beta q\rangle^{2}\langle d e\rangle^{2}}\left(\frac{[f c]^{3}\langle c \alpha\rangle^{3}}{t_{\alpha \beta c}\left[f\left|K_{\alpha \beta}\right| c\right\rangle}+\frac{\langle\alpha e\rangle^{3}[e f]\langle d f\rangle}{\langle c d\rangle\langle e f\rangle^{2}}-\frac{\langle\alpha d\rangle^{3}\langle c e\rangle[d c]\langle c \alpha\rangle}{\langle c d\rangle^{2}\langle e f\rangle\langle f \alpha\rangle}\right)\right. \\
& +\frac{\langle c q\rangle[q|\alpha| q\rangle}{\langle\beta c\rangle\left[q\left|K_{\alpha \beta}\right| c\right\rangle\langle\alpha q\rangle\langle\beta q\rangle\langle d e\rangle^{2}}\left(\frac{[f c]^{3}\langle c \alpha\rangle^{3}}{t_{\alpha \beta c}\left[f\left|K_{\alpha \beta}\right| c\right\rangle}+\frac{\langle\alpha e\rangle^{3}[e f]\langle d f\rangle}{\langle c d\rangle\langle e f\rangle^{2}}-\frac{\langle\alpha d\rangle^{3}\langle c e\rangle[d c]\langle c \alpha\rangle}{\langle c d\rangle^{2}\langle e f\rangle\langle f \alpha\rangle}\right) \\
& +\frac{1}{\langle\beta c\rangle\langle d e\rangle^{2}}\left(\frac{3[\beta f][f|c| \alpha\rangle^{2}}{t_{\alpha \beta c}\left[f\left|K_{\alpha \beta}\right| c\right\rangle}-\frac{\langle\alpha d\rangle^{3}\langle c e\rangle[\beta d]}{\langle c d\rangle^{2}\langle e f\rangle\langle f \alpha\rangle}\right) \\
& +\frac{[\beta f]^{3}}{[\alpha \beta][f \alpha] t_{c d e}}\left(\frac{[\beta c][c d]}{\langle d e\rangle\left[\beta\left|K_{c d}\right| e\right\rangle}-\frac{[d e][e f]}{\langle c d\rangle\left[f\left|K_{\alpha \beta}\right| c\right\rangle}+\frac{[c e]}{\langle c d\rangle\langle d e\rangle}\right) \\
& \left.+\frac{\left[\beta\left|K_{c d}\right| \alpha\right\rangle^{3}}{\langle c d\rangle^{2}\langle e f\rangle\langle f \alpha\rangle t_{\beta c d}\left[\beta\left|K_{c d}\right| e\right\rangle}-\frac{\langle\alpha c\rangle^{3}[\beta c]\langle\beta d\rangle}{\langle\beta c\rangle^{2}\langle c d\rangle^{2}\langle d e\rangle\langle e f\rangle\langle f \alpha\rangle}+\mathcal{O}(\langle\alpha \beta\rangle)\right] .
\end{aligned}
$$

The prefactor in the first line of (C.5) encodes the three-point vertex in diagram 2.8 a and the five-point single minus amplitude on the right can be reconstructed using

$$
\frac{\left[\beta\left|K_{\alpha \beta}\right| X\right\rangle}{\left[\beta\left|K_{\alpha \beta}\right| Y\right\rangle}=\frac{\left[q\left|K_{\alpha \beta}\right| X\right\rangle}{\left[q\left|K_{\alpha \beta}\right| Y\right\rangle}+s_{\alpha \beta} \frac{\langle Y X\rangle[\beta q]}{\left[\beta\left|K_{\alpha \beta}\right| Y\right\rangle\left[q\left|K_{\alpha \beta}\right| Y\right\rangle}+\mathcal{O}\left(s_{\alpha \beta}^{2}\right)
$$


applied to the remainder of the first line:

$$
\begin{gathered}
\frac{1}{\langle\alpha q\rangle}\left(\frac{[f c]^{3}\langle c \alpha\rangle^{3}}{t_{\alpha \beta c}\left[f\left|K_{\alpha \beta}\right| c\right\rangle}+\frac{\langle\alpha e\rangle^{3}[e f]\langle d f\rangle}{\langle c d\rangle\langle e f\rangle^{2}}-\frac{\langle\alpha d\rangle^{3}\langle c e\rangle[d c]\langle c \alpha\rangle}{\langle c d\rangle^{2}\langle e f\rangle\langle f \alpha\rangle}\right) \\
=\langle\alpha q\rangle^{2}\left(-\frac{[f c]^{3}}{t_{\alpha \beta c}\left[f\left|K_{\alpha \beta}\right| c\right\rangle} \frac{\left[\beta\left|K_{\alpha \beta}\right| c\right\rangle^{3}}{\left[\beta\left|K_{\alpha \beta}\right| q\right\rangle^{3}}+\frac{[e f]\langle d f\rangle}{\langle c d\rangle\langle e f\rangle^{2}} \frac{\left[\beta\left|K_{\alpha \beta}\right| e\right\rangle^{3}}{\left[\beta\left|K_{\alpha \beta}\right| q\right\rangle^{3}}-\frac{\langle c e\rangle[d c]}{\langle c d\rangle^{2}\langle e f\rangle} \frac{\left[\beta\left|K_{\alpha \beta}\right| d\right\rangle^{3}}{\left[\beta\left|K_{\alpha \beta}\right| q\right\rangle^{3}} \frac{\left[\beta\left|K_{\alpha \beta}\right| c\right\rangle}{\left[\beta\left|K_{\alpha \beta}\right| f\right\rangle}\right) \\
=\langle\alpha q\rangle^{2}\left(-\frac{[f c]^{3}}{t_{\alpha \beta c}\left[f\left|K_{\alpha \beta}\right| c\right\rangle} \frac{\left[q\left|K_{\alpha \beta}\right| c\right\rangle^{3}}{\left[q\left|K_{\alpha \beta}\right| q\right\rangle^{3}}+\frac{[e f]\langle d f\rangle}{\langle c d\rangle\langle e f\rangle^{2}} \frac{\left[q\left|K_{\alpha \beta}\right| e\right\rangle^{3}}{\left[q\left|K_{\alpha \beta}\right| q\right\rangle^{3}}-\frac{\langle c e\rangle[d c]}{\langle c d\rangle^{2}\langle e f\rangle} \frac{\left[q\left|K_{\alpha \beta}\right| d\right\rangle^{3}}{\left[q\left|K_{\alpha \beta}\right| q\right\rangle^{3}} \frac{\left[q\left|K_{\alpha \beta}\right| c\right\rangle}{\left[q\left|K_{\alpha \beta}\right| f\right\rangle}\right) \\
+\frac{3 s_{\alpha \beta}\langle\alpha q\rangle^{2}[\beta q]}{\left[q\left|K_{\alpha \beta}\right| q\right\rangle}\left(-\frac{[f]^{3}\langle q c\rangle}{t_{\alpha \beta c}\left[f\left|K_{\alpha \beta}\right| c\right\rangle\left[\beta\left|K_{\alpha \beta}\right| q\right\rangle} \frac{\left[\beta\left|K_{\alpha \beta}\right| c\right\rangle^{2}}{\left[\beta\left|K_{\alpha \beta}\right| q\right\rangle^{2}}+\frac{[e f]\langle d f\rangle\langle q e\rangle}{\langle c d\rangle\langle e f\rangle^{2}\left[\beta\left|K_{\alpha \beta}\right| q\right\rangle} \frac{\left[\beta\left|K_{\alpha \beta}\right| e\right\rangle^{2}}{\left[\beta\left|K_{\alpha \beta}\right| q\right\rangle^{2}}\right. \\
\left.-\frac{\left[\beta\left|K_{\alpha \beta}\right| d\right\rangle^{2}}{\langle c d\rangle^{2}\langle e f\rangle} \frac{[d c]}{\left[\beta\left|K_{\alpha \beta}\right| f\right\rangle\left[\beta\left|K_{\alpha \beta}\right| q\right\rangle^{3}}\left(\langle q d\rangle\left[\beta\left|K_{\alpha \beta}\right| c\right\rangle+\frac{\langle f c\rangle\left[q\left|K_{\alpha \beta}\right| q\right\rangle\left[\beta\left|K_{\alpha \beta}\right| d\right\rangle}{3\left[q\left|K_{\alpha \beta}\right| f\right\rangle}\right)\right)
\end{gathered}
$$

and so we obtain,

$$
\begin{aligned}
& \tau_{6: 1 d p}^{(1), \alpha^{-} \beta^{+}}= \frac{i}{3} \frac{\langle q|\alpha \beta| q\rangle\left[q\left|K_{\alpha \beta}\right| q\right\rangle}{s_{\alpha \beta}\left[q\left|K_{\alpha \beta}\right| c\right\rangle\langle d e\rangle^{2}} \frac{\langle\alpha q\rangle^{2}}{\langle\beta q\rangle^{2}}\left(-\frac{[f c]^{3}}{t_{\alpha \beta c}\left[f\left|K_{\alpha \beta}\right| c\right\rangle} \frac{\left[q\left|K_{\alpha \beta}\right| c\right\rangle^{3}}{\left[q\left|K_{\alpha \beta}\right| q\right\rangle^{3}}\right. \\
&\left.+\frac{[e f]\langle d f\rangle}{\langle c d\rangle\langle e f\rangle^{2}} \frac{\left[q\left|K_{\alpha \beta}\right| e\right\rangle^{3}}{\left[q\left|K_{\alpha \beta}\right| q\right\rangle^{3}}-\frac{\langle c e\rangle[d c]}{\langle c d\rangle^{2}\langle e f\rangle} \frac{\left[q\left|K_{\alpha \beta}\right| d\right\rangle^{3}}{\left[q\left|K_{\alpha \beta}\right| q\right\rangle^{3}} \frac{\left[q\left|K_{\alpha \beta}\right| c\right\rangle}{\left[q\left|K_{\alpha \beta}\right| f\right\rangle}\right) .
\end{aligned}
$$

When $K_{\alpha \beta}^{2} \rightarrow 0$ this reduces to 1

$$
\tau_{6: 1 d p}^{(1), \alpha^{-} \beta^{+}}=\frac{\langle\alpha q\rangle^{2}}{\langle\beta q\rangle^{2}} \frac{\langle q|\alpha \beta| q\rangle}{\langle k q\rangle^{2}} \frac{1}{s_{\alpha \beta}} A_{5: 1}^{(1)}\left(k^{-}, c^{+}, d^{+}, e^{+}, f^{+}\right)
$$

which exactly reproduces the first factorisation in fig. 2.8 if we allow $\alpha$ and $\beta$ to

\footnotetext{
${ }^{1}$ From here on $k=K_{\alpha \beta} ; k^{b}$ is defined as in equation (2.183).
} 
be massive. This term will generate the double pole after integration. The order $s_{\alpha \beta}^{1}$ term in (C.7) will contribute to the subleading single pole in $s_{a b}$.

The fourth line in (C.5) contains the $[\alpha \beta]^{-1}$ factor. To extract the factorisation in fig. $2.8 \mathrm{~b}$ we use the following rearrangements:

$$
\begin{aligned}
& \frac{1}{[\alpha \beta]}\left(\frac{[\beta X]}{[\beta Y]}-\frac{\left[k^{b} X\right]}{\left[k^{b} Y\right]}\right)=\frac{1}{[\alpha \beta]} \frac{\left[\beta k^{b}\right][X Y]}{[\beta Y]\left[k^{b} Y\right]}=-\frac{1}{[\alpha \beta]} \frac{\langle q|k| \beta][X Y]}{[\beta Y]\langle q|k| Y]}=-\frac{\langle q \alpha\rangle[X Y]}{[\beta Y]\langle q|k| Y]}, \\
& (\mathrm{C} .10)
\end{aligned}
$$

and

$$
\frac{[\beta f]^{2}[\beta q]\langle\beta \alpha\rangle}{[\alpha q]}=-\frac{\left\langle\alpha k^{b}\right\rangle[\beta q]^{2}}{[\alpha q]\left[k^{b} q\right]}\left[f k^{b}\right]^{2}+s_{\alpha \beta} \frac{[f|q| \alpha\rangle}{[q|k| q\rangle} \frac{\left([f \beta][\beta q]\left[k^{b} q\right]+[\beta q]^{2}\left[f k^{b}\right]\right)}{[\alpha q]\left[k^{b} q\right]} .
$$

Applying (C.10) to the fourth line in (C.5):

$$
\begin{gathered}
\frac{[\beta f]^{3}}{[\alpha \beta][f \alpha] t_{c d e}}\left(\frac{[\beta c][c d]}{\langle d e\rangle\left[\beta\left|K_{c d}\right| e\right\rangle}-\frac{[d e][e f]}{\langle c d\rangle[f|k| c\rangle}+\frac{[c e]}{\langle c d\rangle\langle d e\rangle}\right) \\
=\frac{[\beta f]^{3}}{[\alpha \beta][f \alpha] t_{c d e}}\left(\frac{\left[k^{b} c\right][c d]}{\langle d e\rangle\left[k^{b}\left|K_{c d}\right| e\right\rangle}-\frac{[d e][e f]}{\langle c d\rangle[f|k| c\rangle}+\frac{[c e]}{\langle c d\rangle\langle d e\rangle}\right) \\
+\frac{\langle q \alpha\rangle[\beta f]^{3}[c d]\left[c\left|K_{c d}\right| e\right\rangle}{[f \alpha]\left[\beta\left|K_{c d}\right| e\right\rangle\left\langle e\left|K_{c d} k\right| q\right\rangle\langle d e\rangle t_{c d e}} .
\end{gathered}
$$

Applying (C.11) and (C.12) to the pre-factor:

$$
\frac{[\beta f]^{3}}{[\alpha \beta][f \alpha]}=\frac{[\beta f]^{2}[q f]}{[f \alpha][\alpha q]}+\frac{\left\langle\alpha k^{b}\right\rangle[\beta q]^{2}}{s_{\alpha \beta}[\alpha q]\left[k^{b} q\right]}\left[f k^{b}\right]^{2}-\frac{[f|q| \alpha\rangle}{[q|k| q\rangle} \frac{\left([f \beta][\beta q]\left[k^{b} q\right]+[\beta q]^{2}\left[f k^{b}\right]\right)}{[\alpha q]\left[k^{b} q\right]}
$$


we obtain

$\tau_{6: 1 s b}^{(1), \alpha^{-} \beta^{+}}=-\frac{i}{3} \frac{\langle\alpha q\rangle^{2}}{\langle\beta q\rangle^{2}} \frac{[q|\beta| q\rangle^{2}}{[q|\alpha| q\rangle^{2}} \frac{[q|\alpha \beta| q]}{s_{\alpha \beta}\left[k^{b} q\right]^{2}} \frac{\left[f k^{b}\right]^{2}}{t_{c d e}}\left(\frac{\left[k^{b} c\right][c d]}{\langle d e\rangle\left[k^{b}\left|K_{c d}\right| e\right\rangle}-\frac{[d e][e f]}{\langle c d\rangle[f|k| c\rangle}+\frac{[c e]}{\langle c d\rangle\langle d e\rangle}\right)$.

When $K_{\alpha \beta}^{2} \rightarrow 0$ this reduces to

$$
\tau_{6: 1 s b}^{(1), \alpha^{-} \beta^{+}}=-\frac{[\beta q]^{2}}{[\alpha q]^{2}} \frac{[q|\alpha \beta| q]}{[k q]^{2}} \frac{1}{s_{\alpha \beta}} A_{5: 1}^{(1)}\left(k^{+}, c^{+}, d^{+}, e^{+}, f^{+}\right)
$$

which exactly reproduces the factorisation in fig. 2.8 b. The remaining terms contribute to $\tau_{6}^{\text {n.f. }}$. Explicitly,

$$
\begin{aligned}
\tau_{6: 1 n f}^{(1), \alpha^{-} \beta^{+}}= & \frac{i}{3}\left[\frac{\langle c q\rangle[q|\alpha| q\rangle}{\langle\beta c\rangle[q|k| c\rangle\langle\alpha q\rangle\langle\beta q\rangle\langle d e\rangle^{2}}\left(\frac{[f c]^{3}\langle c \alpha\rangle^{3}}{t_{\alpha \beta c}[f|k| c\rangle}+\frac{\langle\alpha e\rangle^{3}[e f]\langle d f\rangle}{\langle c d\rangle\langle e f\rangle^{2}}-\frac{\langle\alpha d\rangle^{3}\langle c e\rangle[d c]\langle c \alpha\rangle}{\langle c d\rangle^{2}\langle e f\rangle\langle f \alpha\rangle}\right)\right. \\
& +\frac{\langle\alpha q\rangle^{2}}{\langle\beta q\rangle^{2}} \frac{3\langle q|\alpha \beta| q\rangle[\beta q]}{[\beta|k| q\rangle[q|k| c\rangle\langle d e\rangle^{2}}\left(-\frac{[f c]^{3}\langle q c\rangle}{\left.t_{\alpha \beta c}[f|k| c\rangle|k| c\right\rangle^{2}} \frac{[e f]\langle d f\rangle\langle q e\rangle[\beta|k| e\rangle^{2}}{\langle\beta \mid k\rangle^{2}}+\frac{\langle c d\rangle\langle e f\rangle^{2}}{[\beta|k| q\rangle^{2}}\right. \\
& \left.-\frac{\langle c e\rangle[d c]}{\langle c d\rangle^{2}\langle e f\rangle} \frac{\left[\beta\left|K_{\alpha \beta}\right| d\right\rangle^{2}}{\left[\beta\left|K_{\alpha \beta}\right| f\right\rangle\left[\beta\left|K_{\alpha \beta}\right| q\right\rangle^{3}}\left(\langle q d\rangle\left[\beta\left|K_{\alpha \beta}\right| c\right\rangle+\frac{\langle f c\rangle\left[q\left|K_{\alpha \beta}\right| q\right\rangle\left[\beta\left|K_{\alpha \beta}\right| d\right\rangle}{3\left[q\left|K_{\alpha \beta}\right| f\right\rangle}\right)\right) \\
+ & \frac{1}{\langle\beta c\rangle\langle d e\rangle^{2}}\left(\frac{3[\beta f][f|c| \alpha\rangle^{2}}{t_{\alpha \beta c}[f|k| c\rangle}-\frac{\langle\alpha d\rangle^{3}\langle c e\rangle[\beta d]}{\langle c d\rangle^{2}\langle e f\rangle\langle f \alpha\rangle}\right) \\
+ & \frac{\left[\beta\left|K_{c d}\right| \alpha\right\rangle^{3}}{\langle c d\rangle^{2}\langle e f\rangle\langle f \alpha\rangle t_{\beta c d}\left[\beta\left|K_{c d}\right| e\right\rangle}-\frac{\langle\alpha c\rangle^{3}[\beta c]\langle\beta d\rangle}{\langle\beta c\rangle^{2}\langle c d\rangle^{2}\langle d e\rangle\langle e f\rangle\langle f \alpha\rangle} \\
+ & \frac{[\beta f]^{2}[q f]}{[f \alpha][\alpha q]} \frac{1}{t_{c d e}}\left(\frac{\left[k^{b} c\right][c d]}{\langle d e\rangle\left[k^{b}\left|K_{c d}\right| e\right\rangle}-\frac{[d e][e f]}{\langle c d\rangle[f|k| c\rangle}+\frac{[c e]}{\langle c d\rangle\langle d e\rangle}\right) \\
& -\frac{[f|q| \alpha\rangle}{[q|k| q\rangle} \frac{\left([f \beta][\beta q]\left[k^{b} q\right]+[\beta q]^{2}\left[f k^{b}\right]\right)}{[\alpha q]\left[k^{b} q\right] t_{c d e}}\left(\frac{\left[k^{b} c\right][c d]}{\langle d e\rangle\left[k^{b}\left|K_{c d}\right| e\right\rangle}-\frac{[d e][e f]}{\langle c d\rangle[f|k| c\rangle}+\frac{[c e]}{\langle c d\rangle\langle d e\rangle}\right) \\
+ & \left.\frac{\langle q \alpha\rangle[\beta f]^{3}[c d]\left[c\left|K_{c d}\right| e\right\rangle}{[f \alpha]\left[\beta\left|K_{c d}\right| e\right\rangle\left\langle e\left|K_{c d} k\right| q\right\rangle\langle d e\rangle t_{c d e}}\right] \cdot(\mathrm{C} .17)
\end{aligned}
$$


Applying (C.6) and $\alpha+\beta=k$ and applying some minor rearrangements gives

$$
\begin{aligned}
& \tau_{6: 1 n f}^{(1), \alpha^{-} \beta^{+}}=\frac{i}{3} \frac{\langle\alpha q\rangle^{2}}{\langle\beta q\rangle^{2}}\left[[ q | \alpha | q \rangle \frac { \langle c q \rangle } { [ q | k | c \rangle ^ { 2 } [ q | k | q \rangle ^ { 2 } \langle d e \rangle ^ { 2 } } \left(-\frac{[f c]^{3}[q|k| c\rangle^{3}}{t_{\alpha \beta c}[f|k| c\rangle}\right.\right. \\
& \left.+\frac{[e f]\langle d f\rangle[q|k| e\rangle^{3}}{\langle c d\rangle\langle e f\rangle^{2}}-\frac{\langle c e\rangle[d c][q|k| d\rangle^{3}[q|k| c\rangle}{\langle c d\rangle^{2}\langle e f\rangle[q|k| f\rangle}\right)-[c|\beta| d\rangle \frac{[q|k| c\rangle}{\langle c d\rangle^{2}\langle d e\rangle\langle e f\rangle[q|k| f\rangle} \\
& -3[q|\beta| q\rangle \frac{1}{[q|k| c\rangle\langle d e\rangle^{2}}\left(-\frac{[f c]^{3}\langle q c\rangle}{t_{\alpha \beta c}[f|k| c\rangle} \frac{[q|k| c\rangle^{2}}{[q|k| q\rangle^{2}}+\frac{[e f]\langle d f\rangle\langle q e\rangle}{\langle c d\rangle\langle e f\rangle^{2}} \frac{[q|k| e\rangle^{2}}{[q|k| q\rangle^{2}}\right. \\
& \left.-\frac{\langle c e\rangle[d c]}{\langle c d\rangle^{2}\langle e f\rangle} \frac{\left[q\left|K_{\alpha \beta}\right| d\right\rangle^{2}}{\left[q\left|K_{\alpha \beta}\right| f\right\rangle\left[q\left|K_{\alpha \beta}\right| q\right\rangle^{3}}\left(\langle q d\rangle\left[q\left|K_{\alpha \beta}\right| c\right\rangle+\frac{\langle f c\rangle\left[q\left|K_{\alpha \beta}\right| q\right\rangle\left[q\left|K_{\alpha \beta}\right| d\right\rangle}{3\left[q\left|K_{\alpha \beta}\right| f\right\rangle}\right)\right) \\
& -3[f|\beta| q\rangle \frac{[f c]^{2}[q|k| c\rangle}{\langle d e\rangle^{2} t_{\alpha \beta c}[f|k| c\rangle[q|k| q\rangle}-[d|\beta| q\rangle \frac{[q|k| d\rangle^{3}\langle c e\rangle}{\langle c d\rangle^{2}\langle d e\rangle^{2}\langle e f\rangle[q|k| c\rangle[q|k| f\rangle[q|k| q\rangle} \\
& +\left(\frac{[q|\beta| q\rangle^{2}}{[q|\alpha| q\rangle} \frac{[f|k| q\rangle}{[q|k| q\rangle^{2}}+\frac{[f|\beta| q\rangle[q|\beta| q\rangle}{[q|k| q\rangle[q|\alpha| q\rangle}+\frac{[f|\beta| q\rangle^{2}}{[f|\alpha| q\rangle[q|\alpha| q\rangle}\right) \times \\
& \frac{[f q]}{t_{c d e}}\left(\frac{\left[k^{b} c\right][c d]}{\langle d e\rangle\left[k^{b}\left|K_{c d}\right| e\right\rangle}-\frac{[d e][e f]}{\langle c d\rangle[f|k| c\rangle}+\frac{[c e]}{\langle c d\rangle\langle d e\rangle}\right) \\
& \left.-\frac{\left[\beta\left|K_{c d}\right| \alpha\right\rangle^{3}\langle\beta q\rangle^{3}}{\langle f \alpha\rangle t_{\beta c d}\left\langle q\left|\beta K_{c d}\right| e\right\rangle\langle\alpha q\rangle^{2}} \frac{1}{\langle c d\rangle^{2}\langle e f\rangle}-\frac{[f|\beta| q\rangle^{3}}{[f|\alpha| q\rangle\left\langle q\left|\beta K_{c d}\right| e\right\rangle} \frac{[c d]\left[c\left|K_{c d}\right| e\right\rangle}{\left\langle e\left|K_{c d} k\right| q\right\rangle\langle d e\rangle t_{c d e}}\right]
\end{aligned}
$$

Some of the terms can be further expanded to aid integration:

$$
\begin{aligned}
{[c|\beta| d\rangle } & =-[c|\alpha| d\rangle+[c|k| d\rangle=-\frac{\langle d \alpha\rangle}{\langle q \alpha\rangle}[c|\alpha| q\rangle+[c|k| d\rangle \\
\frac{[q|\beta| q\rangle^{2}}{[q|\alpha| q\rangle} & =[q|\alpha| q\rangle-2[q|k| q\rangle+\frac{[q|k| q\rangle^{2}}{[q|\alpha| q\rangle} \\
\frac{[f|\beta| q\rangle[q|\beta| q\rangle}{[q|\alpha| q\rangle} & =-[f|\beta| q\rangle-\frac{[f|\alpha| q\rangle[q|k| q\rangle}{[q|\alpha| q\rangle}+\frac{[f|k| q\rangle[q|k| q\rangle}{[q|\alpha| q\rangle} \\
\frac{[f|\beta| q\rangle^{2}}{[f|\alpha| q\rangle[q|\alpha| q\rangle} & =\frac{[f|\alpha| q\rangle}{[q|\alpha| q\rangle}-2 \frac{[f|k| q\rangle}{[q|\alpha| q\rangle}+\frac{[f|k| q\rangle^{2}}{[f|\alpha| q\rangle[q|\alpha| q\rangle} \\
\frac{[f|\beta| q\rangle^{3}}{[f|\alpha| q\rangle\left\langle q\left|\beta K_{c d}\right| e\right\rangle} & =-\frac{[f|\alpha| q\rangle^{2}}{\left\langle q\left|\beta K_{c d}\right| e\right\rangle}+3 \frac{[f|\alpha| q\rangle[f|k| q\rangle}{\left\langle q\left|\beta K_{c d}\right| e\right\rangle}-3 \frac{[f|k| q\rangle^{2}}{\left\langle q\left|\beta K_{c d}\right| e\right\rangle}+\frac{[f|k| q\rangle^{3}}{[f|\alpha| q\rangle\left\langle q\left|\beta K_{c d}\right| e\right\rangle}
\end{aligned}
$$




\section{DERIVATION OF THE ONE-LOOP SIX-GLUON CURRENT}

The term with $t_{\beta c d}^{-1}$ factor is the most difficult to deal with:

$$
\begin{gathered}
\frac{\left[\beta\left|K_{c d}\right| \alpha\right\rangle^{3}\langle\beta q\rangle^{3}}{\langle f \alpha\rangle t_{\beta c d}\left\langle q\left|\beta K_{c d}\right| e\right\rangle\langle\alpha q\rangle^{2}}=\frac{\left[\beta\left|K_{c d}\right| \beta\right\rangle^{3}\langle\alpha q\rangle}{\langle f \alpha\rangle t_{\beta c d}\left\langle q\left|\beta K_{c d}\right| e\right\rangle}+\mathcal{O}(\langle\alpha \beta\rangle) \\
=-\frac{\left(t_{\beta c d}-s_{c d}\right)^{3}}{t_{\beta c d}\left\langle q\left|\beta K_{c d}\right| e\right\rangle} \frac{[q|k| q\rangle}{[q|k| f\rangle}+\mathcal{O}(\langle\alpha \beta\rangle) \\
=-\frac{[q|k| q\rangle}{[q|k| f\rangle}\left(\frac{\left[\beta\left|K_{c d}\right| \beta\right\rangle^{2}}{\left\langle q\left|\beta K_{c d}\right| e\right\rangle}-\frac{\left[\beta\left|K_{c d}\right| \beta\right\rangle s_{c d}}{\left\langle q\left|\beta K_{c d}\right| e\right\rangle}+\frac{s_{c d}^{2}}{\left\langle q\left|\beta K_{c d}\right| e\right\rangle}-\frac{s_{c d}^{3}}{t_{\beta c d}\left\langle q\left|\beta K_{c d}\right| e\right\rangle}\right)+\mathcal{O}(\langle\alpha \beta\rangle),
\end{gathered}
$$

and

$$
\begin{aligned}
{\left[\beta\left|K_{c d}\right| \beta\right\rangle } & =[c|\beta| c\rangle+[d|\beta| d\rangle=-[c|\alpha| c\rangle-[d|\alpha| d\rangle+([c|k| c\rangle+[d|k| d\rangle) \\
& =\frac{\left\langle\alpha\left|K_{c d} \alpha\right| q\right\rangle}{\langle q \alpha\rangle}+([c|k| c\rangle+[d|k| d\rangle)
\end{aligned}
$$

This yields the final form for $\tau_{6: 1 n f}^{(1), \alpha^{-} \beta^{+}}$. Thus the full amplitude can be written as

$$
A_{6: 1}^{(1)}\left(\alpha^{-}, \beta^{+}, c^{+}, d^{+}, e^{+}, f^{+}\right)=\tau_{6: 1 d p}^{(1), \alpha^{-} \beta^{+}}+\tau_{6: 1 s b}^{(1), \alpha^{-} \beta^{+}}+\tau_{6: 1 n f}^{(1), \alpha^{-} \beta^{+}}+\mathcal{O}(\langle\alpha \beta\rangle),
$$

where

$$
\begin{aligned}
\tau_{6: 1 d p}^{(1), \alpha^{-} \beta^{+}}= & \frac{i}{3} \frac{\langle q|\alpha \beta| q\rangle\left[q\left|K_{\alpha \beta}\right| q\right\rangle}{s_{\alpha \beta}\left[q\left|K_{\alpha \beta}\right| c\right\rangle\langle d e\rangle^{2}} \frac{\langle\alpha q\rangle^{2}}{\langle\beta q\rangle^{2}} \\
& +\frac{[f c]^{3}}{t_{\alpha \beta c}\left[f\left|K_{\alpha \beta}\right| c\right\rangle} \frac{\left[q\left|K_{\alpha \beta}\right| c\right\rangle^{3}}{\left[q\left|K_{\alpha \beta}\right| q\right\rangle^{3}} \\
& \left.\frac{[e f]\langle d f\rangle}{\langle c d\rangle\langle e f\rangle^{2}} \frac{\left[q\left|K_{\alpha \beta}\right| e\right\rangle^{3}}{\left[q\left|K_{\alpha \beta}\right| q\right\rangle^{3}}-\frac{\langle c e\rangle[d c]}{\langle c d\rangle^{2}\langle e f\rangle} \frac{\left[q\left|K_{\alpha \beta}\right| d\right\rangle^{3}}{\left[q\left|K_{\alpha \beta}\right| q\right\rangle^{3}} \frac{\left[q\left|K_{\alpha \beta}\right| c\right\rangle}{\left[q\left|K_{\alpha \beta}\right| f\right\rangle}\right) \\
\tau_{6: 1 s b}^{(1), \alpha^{-} \beta^{+}=}= & -\frac{i}{3} \frac{[q|\beta| q\rangle^{2}}{[q|\alpha| q\rangle^{2}} \frac{[q|\alpha \beta| q]}{\left[q\left|K_{\alpha \beta}\right| q\right\rangle^{2}} \frac{1}{s_{\alpha \beta}} \frac{\left[f\left|K_{\alpha \beta}\right| q\right\rangle^{2}}{t_{c d e}}\left(\frac{\left\langle q\left|K_{\alpha \beta}\right| c\right][c d]}{\langle d e\rangle\left\langle q\left|K_{\alpha \beta} K_{c d}\right| e\right\rangle}-\frac{[d e][e f]}{\langle c d\rangle[f|k| c\rangle}+\frac{[c e]}{\langle c d\rangle\langle d e\rangle}\right)
\end{aligned}
$$


and

$$
\begin{aligned}
& \tau_{6: 1 n f}^{(1), \alpha^{-} \beta^{+}}=\frac{i}{3} \frac{\langle\alpha q\rangle^{2}}{\langle\beta q\rangle^{2}}\left[[ q | \alpha | q \rangle \frac { \langle c q \rangle } { [ q | k | c \rangle ^ { 2 } [ q | k | q \rangle ^ { 2 } \langle d e \rangle ^ { 2 } } \left(-\frac{[f c]^{3}[q|k| c\rangle^{3}}{t_{\alpha \beta c}[f|k| c\rangle}\right.\right. \\
& \left.+\frac{[e f]\langle d f\rangle[q|k| e\rangle^{3}}{\langle c d\rangle\langle e f\rangle^{2}}-\frac{\langle c e\rangle[d c][q|k| d\rangle^{3}[q|k| c\rangle}{\langle c d\rangle^{2}\langle e f\rangle[q|k| f\rangle}\right) \\
& +\left(\frac{\langle d \alpha\rangle}{\langle q \alpha\rangle}[c|\alpha| q\rangle-[c|k| d\rangle\right) \frac{[q|k| c\rangle}{\langle c d\rangle^{2}\langle d e\rangle\langle e f\rangle[q|k| f\rangle} \\
& -3[q|\beta| q\rangle \frac{1}{[q|k| c\rangle\langle d e\rangle^{2}}\left(-\frac{[f c]^{3}\langle q c\rangle}{t_{\alpha \beta c}[f|k| c\rangle} \frac{[q|k| c\rangle^{2}}{[q|k| q\rangle^{2}}+\frac{[e f]\langle d f\rangle\langle q e\rangle}{\langle c d\rangle\langle e f\rangle^{2}} \frac{[q|k| e\rangle^{2}}{[q|k| q\rangle^{2}}\right. \\
& \left.-\frac{\langle c e\rangle[d c]}{\langle c d\rangle^{2}\langle e f\rangle} \frac{[q|k| d\rangle^{2}}{[q|k| f\rangle[q|k| q\rangle^{3}}\left(\langle q d\rangle[q|k| c\rangle+\frac{\langle f c\rangle[q|k| q\rangle[q|k| d\rangle}{3[q|k| f\rangle}\right)\right) \\
& -3[f|\beta| q\rangle \frac{[f c]^{2}[q|k| c\rangle}{\langle d e\rangle^{2} t_{\alpha \beta c}[f|k| c\rangle[q|k| q\rangle}-[d|\beta| q\rangle \frac{[q|k| d\rangle^{3}\langle c e\rangle}{\langle c d\rangle^{2}\langle d e\rangle^{2}\langle e f\rangle[q|k| c\rangle[q|k| f\rangle[q|k| q\rangle} \\
& +\left(\frac{[f|k| q\rangle}{[q|k| q\rangle^{2}}\left([q|\alpha| q\rangle-2[q|k| q\rangle+\frac{[q|k| q\rangle^{2}}{[q|\alpha| q\rangle}\right)+\frac{[f|\alpha| q\rangle}{[q|\alpha| q\rangle}-2 \frac{[f|k| q\rangle}{[q|\alpha| q\rangle}+\frac{[f|k| q\rangle^{2}}{[f|\alpha| q\rangle[q|\alpha| q\rangle}\right. \\
& \left.+\frac{1}{[q|k| q\rangle}\left(-[f|\beta| q\rangle-\frac{[f|\alpha| q\rangle[q|k| q\rangle}{[q|\alpha| q\rangle}+\frac{[f|k| q\rangle[q|k| q\rangle}{[q|\alpha| q\rangle}\right)\right) \times \\
& \frac{[f q]}{t_{c d e}}\left(\frac{\left[k^{b} c\right][c d]}{\langle d e\rangle\left[k^{b}\left|K_{c d}\right| e\right\rangle}-\frac{[d e][e f]}{\langle c d\rangle[f|k| c\rangle}+\frac{[c e]}{\langle c d\rangle\langle d e\rangle}\right) \\
& +\frac{[q|k| q\rangle}{[q|k| f\rangle} \frac{1}{\langle c d\rangle^{2}\langle e f\rangle}\left(\frac{\left(\left\langle\alpha\left|K_{c d} \alpha\right| q\right\rangle+\langle q \alpha\rangle([c|k| c\rangle+[d|k| d\rangle)\right)^{2}}{\langle q \alpha\rangle^{2}\left\langle q\left|\beta K_{c d}\right| e\right\rangle}+\frac{s_{c d}^{2}}{\left\langle q\left|\beta K_{c d}\right| e\right\rangle}\right. \\
& \left.-\frac{s_{c d}^{3}}{t_{\beta c d}\left\langle q\left|\beta K_{c d}\right| e\right\rangle}-\frac{\left(\left\langle\alpha\left|K_{c d} \alpha\right| q\right\rangle+\langle q \alpha\rangle([c|k| c\rangle+[d|k| d\rangle)\right) s_{c d}}{\langle q \alpha\rangle\left\langle q\left|\beta K_{c d}\right| e\right\rangle}\right) \\
& -\frac{[c d]\left[c\left|K_{c d}\right| e\right\rangle}{\left\langle e\left|K_{c d} k\right| q\right\rangle\langle d e\rangle t_{c d e}} \times \\
& \left.\left(-\frac{[f|\alpha| q\rangle^{2}}{\left\langle q\left|\beta K_{c d}\right| e\right\rangle}+3 \frac{[f|\alpha| q\rangle[f|k| q\rangle}{\left\langle q\left|\beta K_{c d}\right| e\right\rangle}-3 \frac{[f|k| q\rangle^{2}}{\left\langle q\left|\beta K_{c d}\right| e\right\rangle}+\frac{[f|k| q\rangle^{3}}{[f|\alpha| q\rangle\left\langle q\left|\beta K_{c d}\right| e\right\rangle}\right)\right],
\end{aligned}
$$

where the expansions (C.19), (C.20) and (C.21) have been applied to (C.18) to produce the final form of $\tau_{6: 1 n f}^{(1), \alpha^{-} \beta^{+}}$. Using this form we take $\alpha$ and $\beta$ off-shell. The $\tau_{6: 1 d p}^{(1), \alpha^{-} \beta^{+}}$and $\tau_{6: 1 s b}^{(1), \alpha^{-} \beta^{+}}$terms then exactly reproduce the two contributions 


\section{DERIVATION OF THE ONE-LOOP SIX-GLUON CURRENT}

in fig. 2.8, while $\tau_{6: 1 n f}^{(1), \alpha^{-} \beta^{+}}$is finite as $s_{\alpha \beta} \longrightarrow 0$. The first two terms then ensure that condition $\mathbf{I}$ is satisfied and all three reproduce the amplitude in the on-shell limit up to terms of order $\langle\alpha \beta\rangle$.

\section{C.1 App 2: Integrations}

In order to determine the contribution from the diagram shown in fig. 4.5 we need to evaluate the integral in (4.71):

$$
\frac{i}{c_{\Gamma}(2 \pi)^{D}} \int d^{D} \ell \frac{[a|\ell| q\rangle[b|\ell| q\rangle}{\langle a q\rangle\langle b q\rangle} \frac{\langle\beta q\rangle^{2}}{\langle\alpha q\rangle^{2}} \tau_{6: 1}^{(1)}\left(\alpha^{-}, \beta^{+}, c^{+}, d^{+}, e^{+}\right)
$$

where we write

$$
\frac{i}{c_{\Gamma}(2 \pi)^{D}} \int d^{D} \ell \frac{[a|\ell| q\rangle[b|\ell| q\rangle}{\langle a q\rangle\langle b q\rangle} \frac{\langle\beta q\rangle^{2}}{\langle\alpha q\rangle^{2}} \equiv \int d \Lambda_{0},
$$

and commonly use the shorthand,

$$
\int d \Lambda_{0}^{\prime}=\int d \Lambda_{0} \frac{\langle\alpha q\rangle^{2}}{\langle\beta q\rangle^{2}}
$$

since the current is often proportional to $\langle\alpha q\rangle^{2} /\langle\beta q\rangle^{2}$ and cancels with its inverse in the integral measure.

Feynman parametrisation is carried out in the usual way,

$$
\begin{aligned}
\frac{1}{\ell^{2} \alpha^{2} \beta^{2}} & =\Gamma(3) \int_{0}^{1} \int_{0}^{1-x_{2}} d x_{1} d x_{2} \frac{1}{\left(\ell^{2}+2 \ell \cdot\left(x_{1} a-x_{2} b\right)\right)^{3}} \\
& =\Gamma(3) \int_{0}^{1} \int_{0}^{1-x_{2}} d x_{1} d x_{2} \frac{1}{\left(\left(\ell+x_{1} a-x_{2} b\right)^{2}+x_{1} x_{2} s_{a b}\right)^{3}}
\end{aligned}
$$

where upon the shift $\ell \rightarrow \ell-x_{1} a+x_{2} b$ the denominator becomes symmetric. The numerators of $C_{d p}$ and $C_{n f}$ contain loop momenta contracted solely with $\lambda_{q}$, thus all quadratic and higher tensor reductions vanish. 


\section{C.1.1 The $C_{d p}$ piece}

Extracting the loop momentum dependent terms from $C_{d p}$ we have

$$
\begin{aligned}
& \int \frac{d^{D} \ell}{\ell^{2} \alpha^{2} \beta^{2}}[a|\ell| q\rangle[b|\ell| q\rangle\langle q|\alpha \beta| q\rangle \\
& =\Gamma(3) \int_{0}^{1} \int_{0}^{1-x_{2}} d x_{1} d x_{2} \int \frac{d^{D} \ell}{\left(\left(\ell+x_{1} a-x_{2} b\right)^{2}+x_{1} x_{2} s_{a b}\right)^{3}}[a|\ell| q\rangle[b|\ell| q\rangle\langle q|(\ell+a) k| q\rangle \\
& =\Gamma(3) \int_{0}^{1} \int_{0}^{1-x_{2}} d x_{1} d x_{2} \int \frac{d^{D} \ell}{\left(\ell^{2}+x_{1} x_{2} s_{a b}\right)^{3}} x_{1} x_{2}[a|b| q\rangle[b|a| q\rangle\left\langle q\left|\left(x_{2} b+\left(1-x_{1}\right) a\right) k\right| q\right\rangle \\
& =\Gamma(3) \frac{-2 \pi^{\frac{D}{2}} i}{\Gamma\left(\frac{D}{2}\right)} \int_{0}^{1} \int_{0}^{1-x_{2}} d x_{1} d x_{2}[a|b| q\rangle[b|a| q\rangle\langle q|a b| q\rangle x_{1} x_{2}\left(1-x_{1}-x_{2}\right) \int_{0}^{\infty} \frac{\ell_{E}^{D-1} d \ell_{E}}{\left(\ell_{E}^{2}-x_{1} x_{2} s_{a b}\right)^{3}} .
\end{aligned}
$$

The integral

$$
\int_{0}^{\infty} \frac{\ell_{E}^{D-1} d \ell_{E}}{\left(\ell_{E}^{2}+\Delta\right)^{3}}=\frac{1}{2} \int_{0}^{\infty} \frac{\left(\ell_{E}^{2}\right)^{\frac{D}{2}-1} d \ell_{E}^{2}}{\left(\ell_{E}^{2}+\Delta\right)^{3}}=\frac{1}{2} \frac{1}{\Delta^{3}} \int_{0}^{\infty} \frac{\left(\ell_{E}^{2}\right)^{\frac{D}{2}-1} d \ell_{E}^{2}}{\left(\ell_{E}^{2}+\Delta\right)^{3}}=\frac{\Delta^{\frac{D}{2}-3}}{2} \frac{\Gamma\left(3-\frac{D}{2}\right) \Gamma\left(\frac{D}{2}\right)}{\Gamma(3)} .
$$

So

$$
\begin{aligned}
\int \frac{d^{D} \ell}{\ell^{2} \alpha^{2} \beta^{2}}[a|\ell| q\rangle[b|\ell| q\rangle\langle q|\alpha \beta| q\rangle= & -i \pi^{\frac{D}{2}} \Gamma\left(3-\frac{D}{2}\right) \frac{[a b]^{3}\langle a q\rangle^{2}\langle b q\rangle^{2}}{s_{a b}^{3-\frac{D}{2}}} \\
& \int_{0}^{1} \int_{0}^{1-x_{2}} d x_{1} d x_{2}\left[\left(1-x_{1}-x_{2}\right)\left(-x_{1} x_{2}\right)^{\frac{D}{2}-2}\right]
\end{aligned}
$$

and

$$
\begin{aligned}
\int_{0}^{1} \int_{0}^{1-x_{2}} d x_{1} d x_{2}\left[\left(1-x_{1}-x_{2}\right)\left(-x_{1} x_{2}\right)^{\frac{D}{2}-2}\right] & =\int_{0}^{1} d x_{1} x_{1}^{\frac{D}{2}-1}\left(1-x_{1}\right)^{\frac{D}{2}}\left(-\frac{1}{\frac{D}{2}-1}+\frac{2}{D}\right) \\
& =\frac{\Gamma\left(\frac{D}{2}-1\right) \Gamma\left(\frac{D}{2}+1\right)}{\Gamma(D)} \frac{-4}{D(D-2)} \\
& =\frac{\Gamma(1-\epsilon) \Gamma(3-\epsilon)}{\Gamma(4-2 \epsilon)} \frac{-1}{(2-\epsilon)(1-\epsilon)} \\
& =\frac{\Gamma(1-\epsilon)^{2}}{\Gamma(4-2 \epsilon)}(-1) ;
\end{aligned}
$$


so that we can simply write

$$
\int d \Lambda_{0}^{\prime}[\langle q|\alpha \beta| q\rangle]=-\frac{1}{6} \frac{[a b]}{\langle a b\rangle}\langle q|a b| q\rangle+\mathcal{O}(\epsilon) .
$$

\section{C.1.2 The $C_{n f}$ piece}

Another algebraic step is needed before integration can easily be carried out on $\tau_{6: 1 n f}^{(1)}$ :

$$
\frac{\langle X \alpha\rangle}{\langle Y \alpha\rangle}=\frac{\langle X a\rangle}{\langle Y a\rangle}+\mathcal{O}(\langle a \alpha\rangle)
$$

implies that

$$
\frac{\left(\left\langle\alpha\left|K_{c d} \alpha\right| q\right\rangle+\langle q \alpha\rangle([c|k| c\rangle+[d|k| d\rangle)\right)}{\langle q \alpha\rangle\left\langle q\left|\beta K_{c d}\right| e\right\rangle}=\frac{\left(\left\langle a\left|K_{c d} \ell\right| q\right\rangle+\langle q a\rangle([c|b| c\rangle+[d|b| d\rangle)\right)}{\langle q a\rangle\left\langle q\left|\beta K_{c d}\right| e\right\rangle}+\mathcal{O}(\langle a \alpha\rangle) .
$$

After this step, the relevant triangle integrals are

$$
\begin{aligned}
\int d \Lambda_{0}^{\prime}[1] & =-\frac{1}{2} \frac{[a b]}{\langle a b\rangle}+\mathcal{O}(\epsilon) \\
\int d \Lambda_{0}^{\prime}[[X|\alpha| q\rangle] & =-\frac{1}{6} \frac{[a b]}{\langle a b\rangle}[X|b+2 a| q\rangle+\mathcal{O}(\epsilon), \\
\int d \Lambda_{0}^{\prime}[[X|\beta| q\rangle] & =-\frac{1}{6} \frac{[a b]}{\langle a b\rangle}[X|2 b+a| q\rangle+\mathcal{O}(\epsilon) .
\end{aligned}
$$

Integrals with extra propagators involve a little bit more subtlety.

Setting $K_{\chi}=\lambda_{q} \bar{\lambda}_{Y}$, some terms in (C.24) have factors which can be promoted to full propagators:

$$
\frac{1}{[Y|\beta| q\rangle}=\frac{1}{\left(\beta+K_{\chi}\right)^{2}}+\mathcal{O}\left(\beta^{2}\right) .
$$

The numerator can be re-written using

$$
\begin{aligned}
{[a|\ell| q\rangle[b|\ell| q\rangle } & =\frac{\left(\alpha^{2}-\ell^{2}\right)\langle q|b \ell| q\rangle+\left(\beta^{2}-\ell^{2}\right)\langle q|a \ell| q\rangle+\ell^{2}\langle q|b a| q\rangle}{\langle a b\rangle} \\
& =\frac{\beta^{2}\langle q|a \ell| q\rangle+\alpha^{2}\langle q|b \ell| q\rangle-\ell^{2}\left\langle q\left|(\ell-b) K_{a b}\right| q\right\rangle}{\langle a b\rangle} .
\end{aligned}
$$


The pole has now been made manifest, thus the integral need not contribute a pole to contribute to the residue. For each term in (C.38) the $\alpha^{2}, \beta^{2}$ and $\ell^{2}$ factors cancel with the propagators in $\int d \Lambda$. For terms involving a single extra loop momentum dependent factor in the denominator this yields a sum of triangle integrals. For the $\alpha^{2}$ term in (C.38)

$$
\begin{aligned}
\frac{\langle q|b \ell| q\rangle}{\ell^{2} \beta^{2}\left(\beta+K_{\chi}\right)^{2}} & \rightarrow \frac{\langle q|b \ell| q\rangle}{\left(\left(\beta-x_{2} b+x_{1} \chi\right)^{2}+x_{1} x_{2}[b|\chi| b\rangle\right)^{3}} \\
& \rightarrow \frac{\left\langle q\left|b\left(x_{2} b-x_{1} \chi\right)\right| q\right\rangle}{\left(\beta^{2}+x_{1} x_{2}[b|\chi| b\rangle\right)^{3}}=0
\end{aligned}
$$

and for the $\ell^{2}$ term

$$
\frac{\left\langle q\left|(\ell-b) K_{a b}\right| q\right\rangle}{\alpha^{2} \beta^{2}\left(\beta+K_{\chi}\right)^{2}} \rightarrow \frac{\left\langle q\left|(\ell-b) K_{a b}\right| q\right\rangle}{\left(\left(\beta-x_{2}(b+a)+x_{1} \chi\right)^{2}-x_{2} s_{a b}+x_{1} x_{2}\left[\chi\left|K_{a b}\right| \chi\right\rangle\right)^{3}} \rightarrow 0
$$

Thus only the $\beta^{2}$ term survives. For the quadratic triangle,

$$
\begin{aligned}
\frac{\langle q|a \ell| q\rangle[X|\ell| q\rangle}{\ell^{2} \alpha^{2}\left(\beta+K_{\chi}\right)^{2}} & \rightarrow \frac{\langle q|a \ell| q\rangle[X|\ell| q\rangle}{\left(\left(\ell+x_{2} a-x_{1}(b+\chi)\right)^{2}+x_{1} x_{2}\left(s_{a b}+[a|\chi| a\rangle\right)-\left(x_{1}-x_{1}^{2}\right)[b|\chi| b\rangle\right)^{3}} \\
& \rightarrow \frac{x_{1}\langle q|a(b+\chi)| q\rangle\left(-x_{2}[X|a| q\rangle+x_{1}[X|b+\chi| q\rangle\right)}{\left(\ell^{2}+x_{1} x_{2}\left(s_{a b}+[a|\chi| a\rangle\right)-\left(x_{1}-x_{1}^{2}\right)[b|\chi| b\rangle\right)^{3}} \\
& \left.=\frac{x_{1}\langle q|a b| q\rangle\left(-x_{2}[X|a| q\rangle+x_{1}[X|b| q\rangle\right)}{\left(\ell^{2}+x_{1} x_{2}[a|\chi| a\rangle-\left(x_{1}-x_{1}^{2}\right)[b|\chi| b\rangle\right)^{3}}+\mathcal{O}\left(s_{a b}\right) ; \quad \quad \text { C. } 41\right)
\end{aligned}
$$

so in this case

$$
\Delta=-x_{1} x_{2}([a|\chi| a\rangle)+\left(x_{1}-x_{1}^{2}\right)[b|\chi| b\rangle .
$$

Carrying out the integration:

$$
\left.\int d \Lambda_{0}^{\prime}\left[\frac{[X|\ell| q\rangle}{\left(\beta+K_{\chi}\right)^{2}}\right]\right|_{\mathbb{Q}}=\frac{1}{2} \frac{[a b]}{\langle a b\rangle} \frac{[X|a| q\rangle}{[a|\chi| a\rangle} .
$$

Note that if the promoted propagator is of the form $\left(\alpha+K_{\chi}\right)^{2}$, then only the $\alpha^{2}$ term survives and the calculation follows in the same way, but with a change of Feynman parametrisation.

Quadratic boxes and quadratic or cubic pentagons lead to nothing but tran- 
scendental functions:

$$
\begin{array}{r}
\left.\int d \Lambda_{0}^{\prime}\left[\frac{1}{(\alpha+X)^{2}}\right]\right|_{\mathbb{Q}}=0, \\
\left.\int d \Lambda_{0}^{\prime}\left[\frac{1}{(\alpha+X)^{2}(\beta+Y)^{2}}\right]\right|_{\mathbb{Q}}=0, \\
\left.\int d \Lambda_{0}^{\prime}\left[\frac{[W|\ell| Z\rangle}{(\alpha+X)^{2}(\beta+Y)^{2}}\right]\right|_{\mathbb{Q}}=0 .
\end{array}
$$

The only other type of term in (C.24) is

$$
\left.\int d \Lambda_{0}^{\prime}\left[\frac{[X|\ell| q\rangle^{2}}{\left(\beta+K_{\chi}\right)^{2}}\right]\right|_{\mathbb{Q}}=-\frac{1}{6} \frac{[a b]}{\langle a b\rangle} \frac{[X|a| q\rangle}{[a|\chi| a\rangle}\left([X|a| q\rangle\left(1-2 \frac{[b|\chi| b\rangle}{[a|\chi| a\rangle}\right)-2[X|b| q\rangle\right)
$$


The integrated non factorising contribution is thus (with $k=K_{a b}$ )

$$
\begin{aligned}
& C_{n f}^{\alpha-\beta+}=-\frac{i}{6} \frac{[a b]}{\langle a b\rangle}\left[\frac{1}{3}[q|b+2 a| q\rangle \frac{\langle c q\rangle}{[q|k| c\rangle^{2}[q|k| q\rangle^{2}\langle d e\rangle^{2}} \times\right. \\
& \left(-\frac{[f c]^{3}[q|k| c\rangle^{3}}{t_{a b c}[f|k| c\rangle}+\frac{[e f]\langle d f\rangle[q|k| e\rangle^{3}}{\langle c d\rangle\langle e f\rangle^{2}}-\frac{\langle c e\rangle[d c][q|k| d\rangle^{3}[q|k| c\rangle}{\langle c d\rangle^{2}\langle e f\rangle[q|k| f\rangle}\right) \\
& +\left(\frac{1}{3} \frac{\langle d a\rangle}{\langle q a\rangle}[c|b+2 a| q\rangle-[c|k| d\rangle\right) \frac{[q|k| c\rangle}{\langle c d\rangle^{2}\langle d e\rangle\langle e f\rangle[q|k| f\rangle} \\
& -[q|2 b+a| q\rangle \frac{1}{[q|k| c\rangle\langle d e\rangle^{2}} \times \\
& \left(-\frac{[f c]^{3}\langle q c\rangle}{t_{\alpha \beta c}[f|k| c\rangle} \frac{[q|k| c\rangle^{2}}{[q|k| q\rangle^{2}}+\frac{[e f]\langle d f\rangle\langle q e\rangle}{\langle c d\rangle\langle e f\rangle^{2}} \frac{[q|k| e\rangle^{2}}{[q|k| q\rangle^{2}}\right. \\
& \left.-\frac{\langle c e\rangle[d c]}{\langle c d\rangle^{2}\langle e f\rangle} \frac{[q|k| d\rangle^{2}}{[q|k| f\rangle[q|k| q\rangle^{3}}\left(\langle q d\rangle[q|k| c\rangle+\frac{\langle f c\rangle[q|k| q\rangle[q|k| d\rangle}{3[q|k| f\rangle}\right)\right) \\
& -[f|2 b+a| q\rangle \frac{[f c]^{2}[q|k| c\rangle}{\langle d e\rangle^{2} t_{a b c}[f|k| c\rangle[q|k| q\rangle}-\frac{1}{3}[d|2 b+a| q\rangle \frac{[q|k| d\rangle^{3}\langle c e\rangle}{\langle c d\rangle^{2}\langle d e\rangle^{2}\langle e f\rangle[q|k| c\rangle[q|k| f\rangle[q|k| q\rangle} \\
& +\left(\frac{[f|k| q\rangle}{[q|k| q\rangle^{2}}\left(\frac{1}{3}[q|b+2 a| q\rangle-2[q|k| q\rangle\right)-\frac{1}{3} \frac{[f|2 b+a| q\rangle}{[q|k| q\rangle}\right) \times \\
& \frac{[f q]}{t_{c d e}}\left(\frac{\langle q|k| c][c d]}{\langle d e\rangle\left\langle q|k| K_{c d} \mid e\right\rangle}-\frac{[d e][e f]}{\langle c d\rangle[f|k| c\rangle}+\frac{[c e]}{\langle c d\rangle\langle d e\rangle}\right) \\
& +\frac{[q|k| q\rangle}{[q|k| f\rangle} \frac{1}{\langle c d\rangle^{2}\langle e f\rangle}\left(\frac{1}{3} \frac{1}{\langle q a\rangle^{2}} \frac{\left\langle a\left|K_{c d} a\right| q\right\rangle}{\left\langle q\left|a K_{c d}\right| e\right\rangle}\left(\left\langle a\left|K_{c d} a\right| q\right\rangle\left(1-2 \frac{\left\langle q\left|b K_{c d}\right| e\right\rangle}{\left\langle q\left|a K_{c d}\right| e\right\rangle}\right)-2\left\langle a\left|K_{c d} b\right| q\right\rangle\right)\right. \\
& \left.-\frac{\left\langle a\left|K_{c d} a\right| q\right\rangle}{\left\langle q\left|a K_{c d}\right| e\right\rangle}\left(\frac{2([c|b| c\rangle+[d|b| d\rangle)-s_{c d}}{\langle q a\rangle}\right)\right) \\
& +\frac{[c d]\left[c\left|K_{c d}\right| e\right\rangle}{\left\langle e\left|K_{c d} k\right| q\right\rangle\langle d e\rangle t_{c d e}} \times \\
& \left.\left.\left(\frac{1}{3} \frac{[f|a| q\rangle}{\left\langle q\left|a K_{c d}\right| e\right\rangle}\left([f|a| q\rangle\left(1-2 \frac{\left\langle q\left|b K_{c d}\right| e\right\rangle}{\left\langle q\left|a K_{c d}\right| e\right\rangle}\right)-2[f|b| q\rangle\right)-(2[f|a| q\rangle-3[f|k| q\rangle) \frac{[f|a| q\rangle}{\left\langle q\left|a K_{c d}\right| e\right\rangle}\right)\right)\right] .
\end{aligned}
$$




\section{DERIVATION OF THE ONE-LOOP SIX-GLUON CURRENT}

\section{App 3: The $C_{s b}$ piece}

Finally, for the square bracket term:

$$
\begin{aligned}
C_{s b}^{\alpha-\beta+} & =-\frac{i}{3} \int d \Lambda_{0}^{\prime}\left[\frac{[q|\beta| q\rangle^{2}}{[q|\alpha| q\rangle^{2}} \frac{[q|\alpha \beta| q]}{s_{\alpha \beta}\left[k^{b} q\right]^{2}} \frac{\left[f k^{b}\right]^{2}}{t_{c d e}}\left(\frac{\left[k^{b} c\right][c d]}{\langle d e\rangle\left[k^{b}\left|K_{c d}\right| e\right\rangle}-\frac{[d e][e f]}{\langle c d\rangle[f|k| c\rangle}+\frac{[c e]}{\langle c d\rangle\langle d e\rangle}\right)\right] \\
& =\int d \Lambda_{0}^{\prime}\left[-\frac{\langle\alpha q\rangle^{2}}{\langle\beta q\rangle^{2}} \frac{[q|\beta| q\rangle^{2}}{[q|\alpha| q\rangle^{2}} \frac{[q|\alpha \beta| q]}{\left[k^{b} q\right]^{2}} \frac{1}{s_{a b}} A_{5: 1}^{(1)}\left(k^{b+}, c^{+}, d^{+}, e^{+}, f^{+}\right)\right] . \quad \text { (C.47) }
\end{aligned}
$$

In this form this term can be summed with the other internal helicity configuration to give:

$C_{s b}=C_{s b}^{\alpha-\beta+}+C_{s b}^{\alpha+\beta-}=\int d \Lambda_{0}^{\prime}\left[-\left(\frac{[q|\beta| q\rangle^{2}}{[q|\alpha| q\rangle^{2}}+\frac{[q|\alpha| q\rangle^{2}}{[q|\beta| q\rangle^{2}}\right) \frac{[q|\alpha \beta| q]}{\left[k^{b} q\right]^{2}} \frac{1}{s_{a b}} A_{5: 1}^{(1)}\left(k^{b+}, c^{+}, d^{+}, e^{+}, f^{+}\right)\right]$.

Using

$$
\begin{aligned}
& \frac{[q|\beta| q\rangle^{2}}{[q|\alpha| q\rangle^{2}}=1-2 \frac{[q|k| q\rangle}{[q|\alpha| q\rangle}+\frac{[q|k| q\rangle^{2}}{[q|\alpha| q\rangle^{2}} \\
& \frac{[q|\alpha| q\rangle^{2}}{[q|\beta| q\rangle^{2}}=1-2 \frac{[q|k| q\rangle}{[q|\beta| q\rangle}+\frac{[q|k| q\rangle^{2}}{[q|\beta| q\rangle^{2}}
\end{aligned}
$$

as the final term in each case corresponds to a cubic pentagon and

$$
\frac{[q|k| q\rangle}{[q|\alpha| q\rangle}+\frac{[q|k| q\rangle}{[q|\beta| q\rangle}=\frac{[q|k| q\rangle^{2}}{[q|\alpha| q\rangle[q|\beta| q\rangle}
$$

also leads to a cubic pentagon only the first term contributes:

$C_{s b}=\int d \Lambda_{0}^{\prime}\left[-2 \frac{[q|\alpha \beta| q]}{\left[k^{b} q\right]^{2}} \frac{1}{s_{a b}} A_{5: 1}^{(1)}\left(k^{b+}, c^{+}, d^{+}, e^{+}, f^{+}\right)\right]+$transcendental functions. 
There is the obvious problem that quadratic terms in the numerator will be present, as the loop momenta are not adjacent to a $\lambda_{q}$ :

$$
\begin{aligned}
{[a|\ell| q\rangle[b|\ell| q\rangle[q|\alpha \beta| q] } & \rightarrow\left[a\left|\ell+x_{2} b\right| q\right\rangle\left[b\left|\ell-x_{1} a\right| q\right\rangle\left[q\left|\left(\ell+\left(1-x_{1}\right) a+x_{2} b\right) K\right| q\right] \\
& \rightarrow \frac{\ell^{2}}{4}\left(([a q]+[b q])[q|K| q\rangle+x_{1} x_{2}[a b]^{2}\langle a q\rangle\langle b q\rangle\right)\left(1-x_{1}-x_{2}\right)[q|a b| q]
\end{aligned}
$$

The logarithmically divergent integral is captured by the cut-constructible piece and thus dropped. The remaining rational integral leads to

$$
\left.C_{s b}\right|_{\mathbb{Q}}=\frac{1}{3} \frac{1}{\langle a b\rangle} \frac{[q a][q b]}{\left[k^{b} q\right]^{2}} A_{5: 1}^{(1)}\left(k^{b+}, c^{+}, d^{+}, e^{+}, f^{+}\right),
$$

which corresponds to the rational part of the one-loop $(+,+;-)$ splitting function [142.

$C_{d p}^{\alpha+\beta-}$ and $C_{n f}^{\alpha+\beta-}$ can be obtained from their respective opposite helicity contributions, $C_{i}^{\alpha-\beta+}$, using the reflection symmetry of the current

$$
C^{\alpha+\beta-}(a, b, c, d, e, f ; q)=C^{\alpha-\beta+}(b, a, f, e, d, c ; q)
$$

The final expression:

$$
C\left(a^{+}, b^{+}, c^{+}, d^{+}, e^{+}, f^{+}, g^{+} ; q\right)=C_{d p}+C_{s b}+C_{n f}
$$

can then be divided by $z$ and shifted to give the residues. 


\section{Appendix D}

\section{5-pt all plus - Infra-Red Divergences}

The singular behaviour of two-loop gluon scattering amplitudes is known from a general analysis [33]. The leading IR singularity for the $n$-point two-loop amplitude is 70$]$

$$
-\frac{s_{a b}^{-\epsilon}}{\epsilon^{2}} f^{a i j} f^{b i k} \times \mathcal{A}_{n}^{(1)}(j, k, \cdots, n),
$$

where $\mathcal{A}_{n}^{(1)}$ is the full-colour one-loop amplitude. We wish to disentangle this simple equation into the colour-ordered partial amplitudes. It will be convenient to use a more list based notation for the partial amplitudes where we use

$$
A_{n}^{(l)}(S)=A_{n}^{(l)}\left(\left\{a_{1}, a_{2}, \cdots a_{n}\right\}\right) \equiv A_{n: 1}^{(l)}\left(a_{1}, a_{2}, \cdots a_{n}\right)
$$

$A_{n}^{(l)}\left(S_{1} ; S_{2}\right)$ for $A_{n: r}^{(l)}$ and $A_{n}^{(l)}\left(S_{1} ; S_{2} ; S_{3}\right)$ for $A_{n: s, t}^{(l)}$. First we define

$$
I_{i, j} \equiv-\frac{\left(s_{i j}\right)^{-\epsilon}}{\epsilon^{2}}
$$

and we have for a list $S=\left\{a_{1}, a_{2}, a_{3}, \cdots, a_{r}\right\}$,

$$
I_{r}[S]=\sum_{i=1}^{r} I_{a_{i}, a_{i+1}}
$$


where the term $I_{a_{r}, a_{r+1}} \equiv I_{a_{r}, a_{1}}$ is included in the sum. We also define $I_{j}\left[S_{1}, S_{2}\right]$ and $I_{k}\left[S_{1}, S_{2}\right]$,

$$
\begin{gathered}
I_{j}\left[S_{1}, S_{2}\right]=I_{j}\left[\left\{a_{1}, a_{2} \cdots a_{r}\right\},\left\{b_{1}, b_{2}, \cdots b_{s}\right\}\right] \equiv\left(I_{a_{1}, a_{r}}+I_{b_{1}, b_{s}}-I_{a_{1}, b_{1}}-I_{a_{r}, b_{s}}\right), \\
I_{k}\left[S_{1}, S_{2}\right]=I_{k}\left[\left\{a_{1}, a_{2} \cdots a_{r}\right\},\left\{b_{1}, b_{2}, \cdots b_{s}\right\}\right] \equiv\left(I_{a_{1}, b_{s}}+I_{b_{1}, a_{r}}-I_{a_{1}, b_{1}}-I_{a_{r}, b_{s}}\right)
\end{gathered}
$$

giving

$$
I_{r}\left[S_{1} \oplus S_{2}\right]=I_{r}\left[S_{1}\right]+I_{r}\left[S_{2}\right]+I_{k}\left[S_{1}, S_{2}\right]-I_{j}\left[S_{1}, S_{2}\right]
$$

where $\left\{a_{1} \cdots a_{r}\right\} \oplus\left\{b_{1} \cdots b_{s}\right\}=\left\{a_{1} \cdots a_{r}, b_{1} \cdots b_{s}\right\}$. In this language the leading and subleading IR singularities at one-loop are

$$
\begin{aligned}
A_{n}^{(1)}(S) & =A_{n}^{(0)}(S) \times I_{r}[S] \\
A_{n}^{(1)}\left(S_{1} ; S_{2}\right) & =\sum_{S_{1}^{\prime} \in C\left(S_{1}\right)} \sum_{S_{2}^{\prime} \in C\left(S_{2}\right)} A_{n}^{(0)}\left(S_{1}^{\prime} \oplus S_{2}^{\prime}\right) \times I_{j}\left[S_{1}^{\prime}, S_{2}^{\prime}\right] .
\end{aligned}
$$

The set $C(S)$ is the set of cyclic permutations of $S$.

At two-loops, we have

$$
\begin{aligned}
A_{n}^{(2)}(S)= & A_{n}^{(1)}(S) \times I_{r}[S], \\
A_{n}^{(2)}\left(S_{1} ; S_{2}\right)= & A_{n}^{(1)}\left(S_{1} ; S_{2}\right) \times\left(I_{r}\left[S_{1}\right]+I_{r}\left[S_{2}\right]\right) \\
& +\sum_{S_{1}^{\prime} \in C\left(S_{1}\right)} \sum_{S_{2}^{\prime} \in C\left(S_{2}\right)} A_{n}^{(1)}\left(S_{1}^{\prime} \oplus S_{2}^{\prime}\right) \times I_{j}\left[S_{1}^{\prime}, S_{2}^{\prime}\right], \\
A_{n}^{(2)}\left(S_{1} ; S_{2} ; S_{3}\right)= & \sum_{S_{2}^{\prime} \in C\left(S_{2}\right)} \sum_{S_{3}^{\prime} \in C\left(S_{3}\right)} A_{n}^{(1)}\left(S_{1} ; S_{2}^{\prime} \oplus S_{3}^{\prime}\right) \times I_{j}\left[S_{2}^{\prime}, S_{3}^{\prime}\right] \\
& +\sum_{S_{1}^{\prime} \in C\left(S_{1}\right)} \sum_{S_{3}^{\prime} \in C\left(S_{3}\right)} A_{n}^{(1)}\left(S_{2} ; S_{1}^{\prime} \oplus S_{3}^{\prime}\right) \times I_{j}\left[S_{1}^{\prime}, S_{3}^{\prime}\right] \\
& +\sum_{S_{1}^{\prime} \in C\left(S_{1}\right)} \sum_{S_{2}^{\prime} \in C\left(S_{2}\right)}^{(1)} A_{n}^{\prime}\left(S_{3} ; S_{1}^{\prime} \oplus S_{2}^{\prime}\right) \times I_{j}\left[S_{1}^{\prime}, S_{2}^{\prime}\right], \\
A_{n, B}^{(2)}(S)= & \sum_{U(S)} A_{n}^{(1)}\left(S_{1}^{\prime} ; S_{2}^{\prime}\right) \times I_{k}\left[S_{1}^{\prime}, S_{2}^{\prime}\right],
\end{aligned}
$$

where $U(S)$ is the set of all distinct pairs of lists satisfying $S_{1}^{\prime} \oplus S_{2}^{\prime} \in C(S)$ where 
the size of $S_{i}^{\prime}$ is greater than one. For example

$$
\begin{aligned}
U(\{1,2,3,4,5\})=\{(\{1,2\},\{3,4,5\}),(\{2,3\},\{4,5,1\}),(\{3,4\},\{5,1,2\}) \\
(\{4,5\},\{1,2,3\}),(\{5,1\},\{2,3,4\})\}
\end{aligned}
$$


D. 5-PT ALL PLUS - INFRA-RED DIVERGENCES 


\section{Bibliography}

[1] B. Feng and M. Luo, "An Introduction to On-shell Recursion Relations," Front. Phys. (Beijing), vol. 7, pp. 533-575, 2012. ix, 3

[2] R. P. Feynman, "Very high-energy collisions of hadrons," Phys. Rev. Lett., vol. 23, pp. 1415-1417, 1969. 1

[3] R. Feynman, "Photon-hadron interactions," 1973. 1]

[4] E. D. Bloom et al., "High-Energy Inelastic e p Scattering at 6-Degrees and 10-Degrees," Phys. Rev. Lett., vol. 23, pp. 930-934, 1969. 1]

[5] M. Breidenbach, J. I. Friedman, H. W. Kendall, E. D. Bloom, D. Coward, H. DeStaebler, J. Drees, L. W. Mo, and R. E. Taylor, "Observed Behavior of Highly Inelastic electron-Proton Scattering," Phys. Rev. Lett., vol. 23, pp. 935-939, 1969. 1

[6] C.-N. Yang and R. L. Mills, "Conservation of Isotopic Spin and Isotopic Gauge Invariance," Phys. Rev., vol. 96, pp. 191-195, 1954. 2

[7] R. Feynman, "The Theory of positrons," Phys. Rev., vol. 76, pp. 749-759, 1949. 2

[8] M. L. Mangano, S. J. Parke, and Z. Xu, "Duality and Multi - Gluon Scattering," Nucl. Phys., vol. B298, pp. 653-672, 1988. 3, 4, 24, 27, 33, 36

[9] F. A. Berends and W. Giele, "The Six Gluon Process as an Example of Weyl-Van Der Waerden Spinor Calculus," Nucl. Phys., vol. B294, pp. 700$732,1987.3,4,24,27,36$ 
[10] J. E. Paton and H.-M. Chan, "Generalized veneziano model with isospin," Nucl. Phys., vol. B10, pp. 516-520, 1969. 3, 24

[11] M. L. Mangano and S. J. Parke, "Quark - Gluon Amplitudes in the Dual Expansion," Nucl. Phys., vol. B299, pp. 673-692, 1988. 4, 33

[12] D. Zeppenfeld, "Diagonalization of Color Factors," Int. J. Mod. Phys., vol. A3, pp. 2175-2179, 1988. 4, 35

[13] V. Del Duca, A. Frizzo, and F. Maltoni, "Factorization of tree QCD amplitudes in the high-energy limit and in the collinear limit," Nucl. Phys., vol. B568, pp. 211-262, 2000. 4, 35, 36

[14] V. Del Duca, L. J. Dixon, and F. Maltoni, "New color decompositions for gauge amplitudes at tree and loop level," Nucl. Phys., vol. B571, pp. 51-70, 2000. 4, 35, 36, 126

[15] S. G. Naculich, "All-loop group-theory constraints for color-ordered SU(N) gauge-theory amplitudes," Phys. Lett., vol. B707, pp. 191-197, 2012. 4, 12, 25, 31, 36, 126, 128, 135, 137

[16] A. C. Edison and S. G. Naculich, "SU(N) group-theory constraints on colorordered five-point amplitudes at all loop orders," Nucl. Phys., vol. B858, pp. 488-501, 2012. 4, 12, 25, 31, 36, 126, 128, 135, 137

[17] S. J. Parke and T. R. Taylor, "Gluonic Two Goes to Four," Nucl. Phys., vol. B269, pp. 410-420, 1986. 4

[18] F. A. Berends and W. T. Giele, "Recursive Calculations for Processes with n Gluons," Nucl. Phys., vol. B306, pp. 759-808, 1988. 4, 9

[19] S. J. Parke and T. R. Taylor, "An Amplitude for $n$ Gluon Scattering," Phys. Rev. Lett., vol. 56, p. 2459, 1986. 4, 44, 47

[20] P. de Causmaecker, Fotonen en Gluonen bij Hoge Energie. PhD thesis, Katholieke Universiteit Leuven, 1983. 4, 37 
[21] P. De Causmaecker, R. Gastmans, W. Troost, and T. T. Wu, "Multiple Bremsstrahlung in Gauge Theories at High-Energies. 1. General Formalism for Quantum Electrodynamics," Nucl. Phys., vol. B206, pp. 53-60, 1982. 4, 37

[22] F. A. Berends, R. Kleiss, P. De Causmaecker, R. Gastmans, W. Troost, and T. T. Wu, "Multiple Bremsstrahlung in Gauge Theories at High-Energies. 2. Single Bremsstrahlung," Nucl. Phys., vol. B206, pp. 61-89, 1982. 4, 37

[23] F. A. Berends, R. Kleiss, P. de Causmaecker, R. Gastmans, W. Troost, and T. T. Wu, "Multiple Bremsstrahlung in Gauge Theories at High-Energies. 4. The Process e+ e- $\longrightarrow$ gamma gamma gamma gamma," Nucl. Phys., vol. B239, pp. 395-409, 1984. 4, 37

[24] F. A. Berends, R. Kleiss, P. de Causmaecker, R. Gastmans, W. Troost, and T. T. Wu, "Multiple Bremsstrahlung in Gauge Theories at High-energies. 3. Finite Mass Effects in Collinear Photon Bremsstrahlung," Nucl. Phys., vol. B239, pp. 382-394, 1984. 4, 37

[25] D. Danckaert, P. De Causmaecker, R. Gastmans, W. Troost, and T. T. $\mathrm{Wu}$, "Four Jet Production in $e^{+} e^{-}$Annihilation," Phys. Lett., vol. 114B, pp. 203-207, 1982. 4, 37

[26] Z. Xu, D.-H. Zhang, and L. Chang, "Helicity Amplitudes for Multiple Bremsstrahlung in Massless Nonabelian Gauge Theories," Nucl. Phys., vol. B291, pp. 392-428, 1987. 4, 37

[27] E. Witten, "Perturbative gauge theory as a string theory in twistor space," Commun. Math. Phys., vol. 252, pp. 189-258, 2004. 4, 5, 7, 20, 39, 43, 48

[28] R. Penrose, "Twistor algebra," J. Math. Phys., vol. 8, p. 345, 1967. 4

[29] F. Cachazo, P. Svrcek, and E. Witten, "MHV vertices and tree amplitudes in gauge theory," JHEP, vol. 09, p. 006, 2004. 5, 53

[30] R. Britto, F. Cachazo, and B. Feng, "Generalized unitarity and one-loop amplitudes in N=4 super-Yang-Mills," Nucl. Phys., vol. B725, pp. 275-305, 2005. 5, 7, 55, 67, 104 
[31] W. T. Giele and E. W. N. Glover, "Higher order corrections to jet crosssections in e+ e- annihilation," Phys. Rev., vol. D46, pp. 1980-2010, 1992. 5

[32] Z. Kunszt, A. Signer, and Z. Trocsanyi, "Singular terms of helicity amplitudes at one loop in QCD and the soft limit of the cross-sections of multiparton processes," Nucl. Phys., vol. B420, pp. 550-564, 1994. 5, 55, 56, 59, 61, 127, 138, 183, 191

[33] S. Catani, "The Singular behavior of QCD amplitudes at two loop order," Phys. Lett., vol. B427, pp. 161-171, 1998. 5, 11, 12, 55, 56, 61, 101, 102, 107, 108, 123, 127, 138, 150, 227

[34] R. Roiban, M. Spradlin, and A. Volovich, "Dissolving N=4 loop amplitudes into QCD tree amplitudes," Phys. Rev. Lett., vol. 94, p. 102002, 2005. 5

[35] R. Britto, F. Cachazo, and B. Feng, "New recursion relations for tree amplitudes of gluons," Nucl. Phys., vol. B715, pp. 499-522, 2005. 5, 8, 9, 45, 48, 165

[36] R. Britto, F. Cachazo, B. Feng, and E. Witten, "Direct proof of tree-level recursion relation in Yang-Mills theory," Phys. Rev. Lett., vol. 94, p. 181602, 2005. 5, 7, 8, 20, 45, 48, 100, 165, 167, 168, 170, 186

[37] K. Risager, "A Direct proof of the CSW rules," JHEP, vol. 12, p. 003, 2005. 5, 48, 53, 79, 100, 151, 168, 170, 195

[38] Z. Bern, L. J. Dixon, and D. A. Kosower, "On-shell recurrence relations for one-loop QCD amplitudes," Phys. Rev., vol. D71, p. 105013, 2005. 5, 8, 9, 71, 74, 111, 119, 132, 209

[39] Z. Bern, L. J. Dixon, and D. A. Kosower, "The last of the finite loop amplitudes in QCD," Phys. Rev. D, vol. 72, p. 125003, 2005. 5

[40] N. Bjerrum-Bohr, D. C. Dunbar, and H. Ita, "Exploiting Twistor Techniques for One-loop QCD Amplitudes," Nucl. Phys. B Proc. Suppl., vol. 160, pp. 66-70, 2006. 5 
[41] S. Badger and E. Glover, "One-loop helicity amplitudes for $\mathrm{H} \longrightarrow$ gluons: The All-minus configuration," Nucl. Phys. B Proc. Suppl., vol. 160, pp. 71$75,2006.5$

[42] C. F. Berger, Z. Bern, L. J. Dixon, D. Forde, and D. A. Kosower, "Onshell unitarity bootstrap for QCD amplitudes," Nucl. Phys. B Proc. Suppl., vol. 160, pp. 261-270, 2006. 5

[43] A. Brandhuber, B. Spence, and G. Travaglini, "Amplitudes in Pure YangMills and MHV Diagrams," JHEP, vol. 02, p. 088, 2007. 5, 6

[44] J. Bedford, A. Brandhuber, B. J. Spence, and G. Travaglini, "A Recursion relation for gravity amplitudes," Nucl. Phys., vol. B721, pp. 98-110, 2005. 6. 166

[45] P. Benincasa and F. Cachazo, "Consistency Conditions on the S-Matrix of Massless Particles," 2007. 6, 169, 179, 182, 186

[46] R. J. Eden, P. V. Landshoff, D. I. Olive, and J. C. Polkinghorne, The analytic S-matrix. Cambridge: Cambridge Univ. Press, 1966. 6, 7, 55, 62, 63, 64, 67, 167, 182

[47] R. E. Cutkosky, "Singularities and discontinuities of Feynman amplitudes," J. Math. Phys., vol. 1, pp. 429-433, 1960. 6, 55, 64

[48] G. 't Hooft and M. J. G. Veltman, "Regularization and Renormalization of Gauge Fields," Nucl. Phys., vol. B44, pp. 189-213, 1972. 6, 55, 56

[49] D. Melrose, "Reduction of Feynman diagrams," Nuovo Cim., vol. 40, pp. $181-213,1965.6,55,57,58,103$

[50] W. van Neerven and J. Vermaseren, "LARGE LOOP INTEGRALS," Phys. Lett. B, vol. 137, pp. 241-244, 1984. 6, 55, 57, 58, 64, 103

[51] G. van Oldenborgh and J. Vermaseren, "New Algorithms for One Loop Integrals," Z. Phys. C, vol. 46, pp. 425-438, 1990. 6, 55, 57, 64, 103 
[52] G. Passarino and M. J. G. Veltman, "One Loop Corrections for e+ e- Annihilation Into mu+ mu- in the Weinberg Model," Nucl. Phys., vol. B160, pp. 151-207, 1979. 7, 55, 57, 98, 103, 182, 183

[53] Z. Bern, L. J. Dixon, D. C. Dunbar, and D. A. Kosower, "Fusing gauge theory tree amplitudes into loop amplitudes," Nucl. Phys., vol. B435, pp. 59101, 1995. 7, 8, 55, 65, 106, 167, 188, 205

[54] Z. Bern, L. J. Dixon, and D. A. Kosower, "Dimensionally regulated one loop integrals," Phys. Lett., vol. B302, pp. 299-308, 1993. [Erratum: Phys. Lett.B318,649(1993)]. 7, 55, 58, 64, 98

[55] Z. Bern, L. J. Dixon, and D. A. Kosower, "Dimensionally regulated pentagon integrals," Nucl. Phys., vol. B412, pp. 751-816, 1994. 7, 55, 58, 64, 75, 205, 206

[56] Z. Bern, L. J. Dixon, D. C. Dunbar, and D. A. Kosower, "One loop n point gauge theory amplitudes, unitarity and collinear limits," Nucl. Phys., vol. B425, pp. 217-260, 1994. 7, 8, 55, 65, 71, 106, 167, 188

[57] Z. Bern, L. J. Dixon, and D. A. Kosower, "One loop amplitudes for e+ eto four partons," Nucl. Phys., vol. B513, pp. 3-86, 1998. 7

[58] Z. Bern, L. J. Dixon, and D. A. Kosower, "Two-loop g $\longrightarrow$ gg splitting amplitudes in QCD," JHEP, vol. 08, p. 012, 2004. 7, 72

[59] D. Forde, "Direct extraction of one-loop integral coefficients," Phys. Rev., vol. D75, p. 125019, 2007. 8, 69, 107

[60] D. C. Dunbar, W. B. Perkins, and E. Warrick, "The Unitarity Method using a Canonical Basis Approach," JHEP, vol. 06, p. 056, 2009. 8, 69, 107

[61] W. van Neerven, "Dimensional Regularization of Mass and Infrared Singularities in Two Loop On-shell Vertex Functions," Nucl. Phys. B, vol. 268, pp. 453-488, 1986. 8, 70

[62] Z. Bern and A. G. Morgan, "Massive loop amplitudes from unitarity," Nucl. Phys., vol. B467, pp. 479-509, 1996. 8, 70, 75 
[63] Z. Bern, L. J. Dixon, and D. A. Kosower, "Progress in one loop QCD computations," Ann. Rev. Nucl. Part. Sci., vol. 46, pp. 109-148, 1996. 8, 70, 75

[64] Z. Bern, L. J. Dixon, D. C. Dunbar, and D. A. Kosower, "One loop selfdual and N=4 superYang-Mills," Phys. Lett., vol. B394, pp. 105-115, 1997. 8, 70, 74

[65] C. Anastasiou, R. Britto, B. Feng, Z. Kunszt, and P. Mastrolia, "Ddimensional unitarity cut method," Phys. Lett., vol. B645, pp. 213-216, 2007. 8, 70

[66] W. T. Giele, Z. Kunszt, and K. Melnikov, "Full one-loop amplitudes from tree amplitudes," JHEP, vol. 04, p. 049, 2008. 8, 70

[67] G. Ossola, C. G. Papadopoulos, and R. Pittau, "Reducing full one-loop amplitudes to scalar integrals at the integrand level," Nucl. Phys., vol. B763, pp. 147-169, 2007. 8, 10, 70, 98

[68] C. Anastasiou, R. Britto, B. Feng, Z. Kunszt, and P. Mastrolia, "Unitarity cuts and Reduction to master integrals in d dimensions for one-loop amplitudes," JHEP, vol. 03, p. 111, 2007. 8, 70

[69] Z. Bern, L. J. Dixon, and D. A. Kosower, "Unitarity based techniques for one loop calculations in QCD," Nucl. Phys. Proc. Suppl., vol. 51C, pp. 243249, 1996. [,243(1996)]. 8, 75

[70] Z. Bern, L. J. Dixon, and D. A. Kosower, "A Two loop four gluon helicity amplitude in QCD," JHEP, vol. 01, p. 027, 2000. 8, 10, 75, 97, 126, 152, 227

[71] S. D. Badger, "Direct Extraction Of One Loop Rational Terms," JHEP, vol. 01, p. 049, 2009. 8, 70

[72] M. T. Grisaru, H. N. Pendleton, and P. van Nieuwenhuizen, "Supergravity and the S Matrix," Phys. Rev., vol. D15, p. 996, 1977. 8, 73, 101 
[73] M. T. Grisaru and H. N. Pendleton, "Some Properties of Scattering Amplitudes in Supersymmetric Theories," Nucl. Phys., vol. B124, pp. 81-92, 1977. 8, 73, 101

[74] S. J. Parke and T. R. Taylor, "Perturbative QCD Utilizing Extended Supersymmetry," Phys. Lett., vol. 157B, p. 81, 1985. [Erratum: Phys. Lett.B174,465(1986)]. 8, 73

[75] M. L. Mangano and S. J. Parke, "Multiparton amplitudes in gauge theories," Phys. Rept., vol. 200, pp. 301-367, 1991. 8

[76] L. J. Dixon, "Calculating scattering amplitudes efficiently," in $Q C D$ and beyond. Proceedings, Theoretical Advanced Study Institute in Elementary Particle Physics, TASI-95, Boulder, USA, June 4-30, 1995, pp. 539-584, 1996. 8

[77] Z. Bern, G. Chalmers, L. J. Dixon, and D. A. Kosower, "One loop N gluon amplitudes with maximal helicity violation via collinear limits," Phys. Rev. Lett., vol. 72, pp. 2134-2137, 1994. 8, 71, 73, 101, 130

[78] Z. Bern, L. J. Dixon, and D. A. Kosower, "New QCD results from string theory," in International Conference on Strings 93 Berkeley, California, May 24-29, 1993, pp. 0190-204, 1993. 8, 71, 73

[79] G. Mahlon, "Multi - gluon helicity amplitudes involving a quark loop," Phys. Rev., vol. D49, pp. 4438-4453, 1994. 8, 9, 73

[80] G. Mahlon, "One loop multi - photon helicity amplitudes," Phys. Rev., vol. D49, pp. 2197-2210, 1994. 8

[81] D. C. Dunbar, J. H. Ettle, and W. B. Perkins, "Augmented Recursion For One-loop Gravity Amplitudes," JHEP, vol. 06, p. 027, 2010. 9, 55, 76, 80, 99

[82] D. C. Dunbar, J. H. Ettle, and W. B. Perkins, "Augmented Recursion For One-loop Amplitudes," Nucl. Phys. Proc. Suppl., vol. 205-206, pp. 74-79, 2010. 9 
[83] S. D. Alston, D. C. Dunbar, and W. B. Perkins, "Complex Factorisation and Recursion for One-Loop Amplitudes," Phys. Rev., vol. D86, p. 085022, 2012. 9, 177

[84] S. D. Alston, D. C. Dunbar, and W. B. Perkins, " $n$-point amplitudes with a single negative-helicity graviton," Phys. Rev., vol. D92, no. 6, p. 065024, 2015. 9, 55, 76, 80, 99, 155

[85] D. C. Dunbar and W. B. Perkins, " $\mathcal{N}=4$ supergravity next-to-maximallyhelicity-violating six-point one-loop amplitude," Phys. Rev., vol. D94, no. 12 , p. $125027,2016.9,55,76,80,83,99$

[86] D. C. Dunbar and W. B. Perkins, "Two-loop five-point all plus helicity Yang-Mills amplitude," Phys. Rev., vol. D93, no. 8, p. 085029, 2016. 9, 10, 55, 76, 80, 84, 89, 98, 155, 156, 160, 161

[87] D. C. Dunbar, G. R. Jehu, and W. B. Perkins, "Two-loop six gluon all plus helicity amplitude," Phys. Rev. Lett., vol. 117, no. 6, p. 061602, 2016. 9, 10, 12, 55, 76, 80, 99, 102, 108, 118, 123

[88] D. C. Dunbar, J. H. Godwin, G. R. Jehu, and W. B. Perkins, "Analytic all-plus-helicity gluon amplitudes in QCD," Phys. Rev., vol. D96, no. 11, p. $116013,2017.9,10,11,55,72,76,80,99,151,155$

[89] D. C. Dunbar, J. H. Godwin, W. B. Perkins, and J. M. W. Strong, "Color Dressed Unitarity and Recursion for Yang-Mills Two-Loop All-Plus Amplitudes," Phys. Rev., vol. D101, no. 1, p. 016009, 2020. 9, 11, 34, 42, 55, 72, 76, 80, 84, 89, 127

[90] D. C. Dunbar, W. B. Perkins, and J. M. Strong, "n-point QCD two-loop amplitude," Phys. Rev. D, vol. 101, no. 7, p. 076001, 2020. 9, 11, 163

[91] A. R. Dalgleish, D. C. Dunbar, W. B. Perkins, and J. M. Strong, "The Full Color Two-Loop Six-Gluon All-Plus Helicity Amplitude," 2 2020. 9, 11, 163

[92] Z. Bern, A. De Freitas, and L. J. Dixon, "Two loop helicity amplitudes for gluon-gluon scattering in QCD and supersymmetric Yang-Mills theory," JHEP, vol. 03, p. 018, 2002. 10, 97, 126, 152, 162 
[93] E. W. N. Glover, C. Oleari, and M. E. Tejeda-Yeomans, "Two loop QCD corrections to gluon-gluon scattering," Nucl. Phys., vol. B605, pp. 467-485, 2001. 10, 97, 126

[94] S. Badger, H. Frellesvig, and Y. Zhang, "A Two-Loop Five-Gluon Helicity Amplitude in QCD," JHEP, vol. 12, p. 045, 2013. 10, 70, 98, 126

[95] S. Badger, G. Mogull, A. Ochirov, and D. O'Connell, "A Complete TwoLoop, Five-Gluon Helicity Amplitude in Yang-Mills Theory," JHEP, vol. 10, p. $064,2015.10,70,98,126$

[96] T. Gehrmann, J. M. Henn, and N. A. Lo Presti, "Analytic form of the two-loop planar five-gluon all-plus-helicity amplitude in QCD," Phys. Rev. Lett., vol. 116, no. 6, p. 062001, 2016. [Erratum: Phys. Rev. Lett.116,no.18,189903(2016)]. 10, 56, 70, 98, 101, 146

[97] J. M. Henn, "Multiloop integrals in dimensional regularization made simple," Phys. Rev. Lett., vol. 110, p. 251601, 2013. 10, 75, 98, 101, 108

[98] S. Badger, G. Mogull, and T. Peraro, "Local integrands for two-loop all-plus Yang-Mills amplitudes," JHEP, vol. 08, p. 063, 2016. 10, 118

[99] A. Ochirov and B. Page, "Full Colour for Loop Amplitudes in Yang-Mills Theory," JHEP, vol. 02, p. 100, 2017. 10, 70

[100] S. Abreu, F. Febres Cordero, H. Ita, M. Jaquier, B. Page, and M. Zeng, "Two-Loop Four-Gluon Amplitudes from Numerical Unitarity," Phys. Rev. Lett., vol. 119, no. 14, p. 142001, 2017. 10, 70

[101] S. Badger, C. Brønnum-Hansen, H. B. Hartanto, and T. Peraro, "First look at two-loop five-gluon scattering in QCD," Phys. Rev. Lett., vol. 120, no. 9, p. 092001, 2018. 10, 70

[102] S. Abreu, F. Febres Cordero, H. Ita, B. Page, and M. Zeng, "Planar Two-Loop Five-Gluon Amplitudes from Numerical Unitarity," Phys. Rev., vol. D97, no. 11, p. 116014, 2018. 10, 70 
[103] H. A. Chawdhry, M. A. Lim, and A. Mitov, "Two-loop five-point massless QCD amplitudes within the integration-by-parts approach," Phys. Rev., vol. D99, no. 7, p. 076011, 2019. 10, 126

[104] S. Abreu, F. Febres Cordero, H. Ita, B. Page, and M. Zeng, "Five-Point Two-Loop Amplitudes from Numerical Unitarity," PoS, vol. LL2018, p. 016, 2018. 10

[105] S. Abreu, F. Febres Cordero, H. Ita, B. Page, and V. Sotnikov, "Planar TwoLoop Five-Parton Amplitudes from Numerical Unitarity," JHEP, vol. 11, p. 116, 2018. 10, 70

[106] S. Badger, C. Brønnum-Hansen, H. B. Hartanto, and T. Peraro, "Analytic helicity amplitudes for two-loop five-gluon scattering: the single-minus case," JHEP, vol. 01, p. 186, 2019. 10, 70

[107] S. Abreu, J. Dormans, F. Febres Cordero, H. Ita, and B. Page, "Analytic Form of Planar Two-Loop Five-Gluon Scattering Amplitudes in QCD," Phys. Rev. Lett., vol. 122, no. 8, p. 082002, 2019. 10

[108] D. Chicherin, T. Gehrmann, J. M. Henn, P. Wasser, Y. Zhang, and S. Zoia, "Analytic result for a two-loop five-particle amplitude," Phys. Rev. Lett., vol. 122 , no. 12 , p. $121602,2019$. 10, 70

[109] S. Abreu, J. Dormans, F. Febres Cordero, H. Ita, B. Page, and V. Sotnikov, "Analytic Form of the Planar Two-Loop Five-Parton Scattering Amplitudes in QCD," JHEP, vol. 05, p. 084, 2019. 10, 70

[110] S. Badger, D. Chicherin, T. Gehrmann, G. Heinrich, J. M. Henn, T. Peraro, P. Wasser, Y. Zhang, and S. Zoia, "Analytic form of the full two-loop five-gluon all-plus helicity amplitude," Phys. Rev. Lett., vol. 123, no. 7, p. $071601,2019.10,12,70,126,128,146,162,163$

[111] D. C. Dunbar, G. R. Jehu, and W. B. Perkins, "The two-loop n-point allplus helicity amplitude," Phys. Rev., vol. D93, no. 12, p. 125006, 2016. 11, 12, 75, 99, 102, 103, 105, 106, 107, 108, 150 
[112] D. C. Dunbar, J. H. Godwin, G. R. Jehu, and W. B. Perkins, "Diagrammar in an Extended Theory of Gravity," Phys. Lett., vol. B771, pp. 230-234, 2017. 11, 166, 182, 185, 192, 195

[113] D. C. Dunbar, J. H. Godwin, G. R. Jehu, and W. B. Perkins, "Loop Amplitudes in an Extended Gravity Theory," Phys. Lett., vol. B780, pp. 41-47, 2018. 11, 182, 195

[114] C. D. White, "Factorization Properties of Soft Graviton Amplitudes," JHEP, vol. 05, p. 060, 2011. 13, 47, 167, 176

[115] F. Cachazo and A. Strominger, "Evidence for a New Soft Graviton Theorem," 2014. 13, 47, 167, 176, 177

[116] T. He, V. Lysov, P. Mitra, and A. Strominger, "BMS supertranslations and Weinberg's soft graviton theorem," JHEP, vol. 05, p. 151, 2015. 13, 47, 167, 176

[117] S. Weinberg, "Infrared photons and gravitons," Phys. Rev., vol. 140, pp. B516-B524, 1965. 13, 47, 167, 183, 194

[118] R. Ellis, W. Stirling, and B. Webber, QCD and collider physics, vol. 8. Cambridge University Press, 2 2011. 17

[119] V. Gribov and L. Lipatov, "Deep inelastic e p scattering in perturbation theory," Sov. J. Nucl. Phys., vol. 15, pp. 438-450, 1972. 17

[120] Y. L. Dokshitzer, "Calculation of the Structure Functions for Deep Inelastic Scattering and e+ e- Annihilation by Perturbation Theory in Quantum Chromodynamics.," Sov. Phys. JETP, vol. 46, pp. 641-653, 1977. 17

[121] G. Altarelli and G. Parisi, "Asymptotic Freedom in Parton Language," Nucl. Phys. B, vol. 126, pp. 298-318, 1977. 17

[122] C. Schwinn and S. Weinzierl, "Scalar diagrammatic rules for Born amplitudes in QCD," JHEP, vol. 05, p. 006, 2005. 20, 41, 80, 100 
[123] H. Kawai, D. C. Lewellen, and S. H. H. Tye, "A Relation Between Tree Amplitudes of Closed and Open Strings," Nucl. Phys., vol. B269, pp. 1-23, 1986. 21, 175

[124] M. J. G. Veltman, "Quantum Theory of Gravitation," Conf. Proc., vol. C7507281, pp. 265-327, 1975. 22

[125] H. Elvang and Y.-t. Huang, Scattering Amplitudes in Gauge Theory and Gravity. Cambridge University Press, 4 2015. 22

[126] S. Weinzierl, "Tales of 1001 Gluons," Phys. Rept., vol. 676, pp. 1-101, 2017. 22

[127] G. 't Hooft, "Dimensional regularization and the renormalization group," Nucl. Phys., vol. B61, pp. 455-468, 1973. 23

[128] Z. Bern and D. A. Kosower, "Color decomposition of one loop amplitudes in gauge theories," Nucl. Phys., vol. B362, pp. 389-448, 1991. 25, 29, 32, $34,36,125,130,184$

[129] R. Kleiss and H. Kuijf, "Multi - Gluon Cross-sections and Five Jet Production at Hadron Colliders," Nucl. Phys., vol. B312, pp. 616-644, 1989. 28, 36

[130] Z. Bern, J. J. M. Carrasco, and H. Johansson, "New Relations for GaugeTheory Amplitudes," Phys. Rev., vol. D78, p. 085011, 2008. 28, 36

[131] G. 't Hooft, "A Planar Diagram Theory for Strong Interactions," Nucl. Phys., vol. B72, p. 461, 1974. [,337(1973)]. 29

[132] D. A. Kosower, "Light Cone Recurrence Relations for QCD Amplitudes," Nucl. Phys., vol. B335, pp. 23-44, 1990. 41, 80

[133] M. L. Mangano and S. J. Parke, "Soft and Collinear Behavior of Dual Amplitudes," in High-energy physics. Proceedings, International Europhysics Conference, Uppsala, Sweden, June 25 - July 1, 1987. Vols. 1, 2, 1987. 47, 72 
[134] F. A. Berends and W. T. Giele, "Multiple Soft Gluon Radiation in Parton Processes," Nucl. Phys., vol. B313, pp. 595-633, 1989. 47, 72

[135] T. Cohen, H. Elvang, and M. Kiermaier, "On-shell constructibility of tree amplitudes in general field theories," JHEP, vol. 04, p. 053, 2011. 48, 173, 176, 185, 192

[136] N. Arkani-Hamed and J. Kaplan, "On Tree Amplitudes in Gauge Theory and Gravity," JHEP, vol. 04, p. 076, 2008. 52

[137] Z. Bern, J. J. Carrasco, D. Forde, H. Ita, and H. Johansson, "Unexpected Cancellations in Gravity Theories," Phys. Rev., vol. D77, p. 025010, 2008. 52

[138] R. G. Stuart, "Algebraic Reduction of One Loop Feynman Diagrams to Scalar Integrals," Comput. Phys. Commun., vol. 48, pp. 367-389, 1988. 55, 58

[139] R. G. Stuart and A. Gongora, "Algebraic Reduction of One Loop Feynman Diagrams to Scalar Integrals. 2.," Comput. Phys. Commun., vol. 56, pp. 337-350, 1990. 55, 58

[140] D. A. Kosower, "All order collinear behavior in gauge theories," Nucl. Phys., vol. B552, pp. 319-336, 1999. 70, 71, 72

[141] E. Nigel Glover and C. Williams, "One-Loop Gluonic Amplitudes from Single Unitarity Cuts," JHEP, vol. 12, p. 067, 2008. 70

[142] Z. Bern and G. Chalmers, "Factorization in one loop gauge theory," Nucl. Phys., vol. B447, pp. 465-518, 1995. 71, 72, 225

[143] S. Catani and M. Grazzini, "The soft gluon current at one loop order," Nucl. Phys., vol. B591, pp. 435-454, 2000. 72

[144] S. D. Badger and E. W. N. Glover, "Two loop splitting functions in QCD," JHEP, vol. 07, p. 040, 2004. 72 
[145] C. Duhr and T. Gehrmann, "The two-loop soft current in dimensional regularization," Phys. Lett., vol. B727, pp. 452-455, 2013. 72

[146] Y. Li and H. X. Zhu, "Single soft gluon emission at two loops," JHEP, vol. 11, p. $080,2013.72$

[147] L. J. Dixon, E. Herrmann, K. Yan, and H. X. Zhu, "Soft gluon emission at two loops in full color," 2019. 72

[148] Z. Bern, L. J. Dixon, and D. A. Kosower, "One loop corrections to five gluon amplitudes," Phys. Rev. Lett., vol. 70, pp. 2677-2680, 1993. 84, 131

[149] E. Remiddi, "Differential equations for Feynman graph amplitudes," Nuovo Cim., vol. A110, pp. 1435-1452, 1997. 98

[150] J. M. Drummond, J. M. Henn, and J. Trnka, "New differential equations for on-shell loop integrals," JHEP, vol. 04, p. 083, 2011. 98

[151] K. Chetyrkin and F. Tkachov, "Integration by Parts: The Algorithm to Calculate beta Functions in 4 Loops," Nucl. Phys. B, vol. 192, pp. 159-204, 1981. 98

[152] C. G. Papadopoulos, D. Tommasini, and C. Wever, "The Pentabox Master Integrals with the Simplified Differential Equations approach," JHEP, vol. 04, p. 078, 2016. 98

[153] E. Remiddi and L. Tancredi, "Differential equations and dispersion relations for Feynman amplitudes. The two-loop massive sunrise and the kite integral," Nucl. Phys., vol. B907, pp. 400-444, 2016. 98

[154] J. Ablinger, J. Blümlein, A. De Freitas, M. van Hoeij, E. Imamoglu, C. G. Raab, C. S. Radu, and C. Schneider, "Iterated Elliptic and Hypergeometric Integrals for Feynman Diagrams," J. Math. Phys., vol. 59, no. 6, p. 062305, 2018. 98 
[155] J. L. Bourjaily, A. J. McLeod, M. Spradlin, M. von Hippel, and M. Wilhelm, "Elliptic Double-Box Integrals: Massless Scattering Amplitudes beyond Polylogarithms," Phys. Rev. Lett., vol. 120, no. 12, p. 121603, 2018. 98

[156] T. Gehrmann, J. M. Henn, and N. A. Lo Presti, "Pentagon functions for massless planar scattering amplitudes," JHEP, vol. 10, p. 103, 2018. 98

[157] C. Papadopoulos and C. Wever, "Progress on two-loop five-point Master Integrals," PoS, vol. LL2018, p. 015, 2018. 98

[158] D. Chicherin, T. Gehrmann, J. M. Henn, N. A. Lo Presti, V. Mitev, and P. Wasser, "Analytic result for the nonplanar hexa-box integrals," JHEP, vol. 03, p. 042, 2019. 98

[159] D. Chicherin, T. Gehrmann, J. M. Henn, P. Wasser, Y. Zhang, and S. Zoia, "All Master Integrals for Three-Jet Production at Next-to-Next-to-Leading Order," Phys. Rev. Lett., vol. 123, no. 4, p. 041603, 2019. 98, 126

[160] Z. Bern, J. S. Rozowsky, and B. Yan, "Two loop four gluon amplitudes in N=4 superYang-Mills," Phys. Lett., vol. B401, pp. 273-282, 1997. 135

[161] F. Cachazo and P. Svrcek, "Tree level recursion relations in general relativity," 2005. 166

[162] N. E. J. Bjerrum-Bohr, D. C. Dunbar, H. Ita, W. B. Perkins, and K. Risager, "MHV-vertices for gravity amplitudes," JHEP, vol. 01, p. 009, 2006. 166, 195

[163] P. Benincasa, C. Boucher-Veronneau, and F. Cachazo, "Taming Tree Amplitudes In General Relativity," JHEP, vol. 11, p. 057, 2007. 166, 168, 195

[164] D. C. Dunbar, G. R. Jehu, and W. B. Perkins, "Two-Loop Gravity amplitudes from four dimensional Unitarity," Phys. Rev., vol. D95, no. 4, p. $046012,2017.166,173,192$ 
[165] G. 't Hooft and M. J. G. Veltman, "DIAGRAMMAR," NATO Sci. Ser. B, vol. 4, pp. 177-322, 1974. [,177(1973)]. 167, 181

[166] N. Arkani-Hamed and J. Trnka, "The Amplituhedron," JHEP, vol. 10, p. 030, 2014. 167

[167] N. Arkani-Hamed, L. Rodina, and J. Trnka, "Locality and Unitarity of Scattering Amplitudes from Singularities and Gauge Invariance," Phys. Rev. Lett., vol. 120, no. 23, p. 231602, 2018. 167

[168] P. van Nieuwenhuizen and C. C. Wu, "On Integral Relations for Invariants Constructed from Three Riemann Tensors and their Applications in Quantum Gravity," J. Math. Phys., vol. 18, p. 182, 1977. 173, 192

[169] M. T. Grisaru, "Two Loop Renormalizability of Supergravity," Phys. Lett., vol. 66B, pp. $75-76,1977.173$

[170] E. Tomboulis, "On the Two Loop Divergences of Supersymmetric Gravitation," Phys. Lett., vol. 67B, pp. 417-420, 1977. 173

[171] Z. Bern, H.-H. Chi, L. Dixon, and A. Edison, "Two-Loop Renormalization of Quantum Gravity Simplified," Phys. Rev., vol. D95, no. 4, p. 046013, 2017. 173, 192

[172] D. C. Dunbar and N. W. P. Turner, "Gravity and form scattering and renormalization of gravity in six-dimensions and eight-dimensions," Class. Quant. Grav., vol. 20, pp. 2293-2324, 2003. 173, 192

[173] J. Broedel and L. J. Dixon, "Color-kinematics duality and double-copy construction for amplitudes from higher-dimension operators," JHEP, vol. 10, p. 091, 2012. 175, 176, 177, 185, 186

[174] Z. Bern, S. Davies, and J. Nohle, "On Loop Corrections to Subleading Soft Behavior of Gluons and Gravitons," Phys. Rev., vol. D90, no. 8, p. 085015, 2014. 177 
[175] J. Broedel, M. de Leeuw, J. Plefka, and M. Rosso, "Constraining subleading soft gluon and graviton theorems," Phys. Rev., vol. D90, no. 6, p. 065024, 2014. 177

[176] H. Bondi, M. G. J. van der Burg, and A. W. K. Metzner, "Gravitational waves in general relativity. 7. Waves from axisymmetric isolated systems," Proc. Roy. Soc. Lond., vol. A269, pp. 21-52, 1962. 177

[177] S. He, Y.-t. Huang, and C. Wen, "Loop Corrections to Soft Theorems in Gauge Theories and Gravity," JHEP, vol. 12, p. 115, 2014. 177

[178] M. Bianchi, S. He, Y.-t. Huang, and C. Wen, "More on Soft Theorems: Trees, Loops and Strings," Phys. Rev., vol. D92, no. 6, p. 065022, 2015. 177

[179] S. A. Fulling, R. C. King, B. G. Wybourne, and C. J. Cummins, "Normal forms for tensor polynomials. 1: The Riemann tensor," Class. Quant. Grav., vol. 9, pp. 1151-1197, 1992. 178

[180] S. Narison and R. Tarrach, "Higher Dimensional Renormalization Group Invariant Vacuum Condensates in Quantum Chromodynamics," Phys. Lett., vol. 125B, pp. 217-222, 1983. 182, 191

[181] A. Yu. Morozov, "MATRIX OF MIXING OF SCALAR AND VECTOR MESONS OF DIMENSION D $<=8$ IN QCD. (IN RUSSIAN)," Sov. J. Nucl. Phys., vol. 40, p. 505, 1984. [Yad. Fiz.40,788(1984)]. 182, 191

[182] C. W. Bauer and A. V. Manohar, "Renormalization group scaling of the 1/m**2 HQET Lagrangian," Phys. Rev., vol. D57, pp. 337-343, 1998. 182, 191

[183] J. A. Gracey, "Classification and one loop renormalization of dimensionsix and dimension-eight operators in quantum gluodynamics," Nucl. Phys., vol. B634, pp. 192-208, 2002. [Erratum: Nucl. Phys.B696,295(2004)]. 182, 191 
[184] D. C. Dunbar and P. S. Norridge, "Infinities within graviton scattering amplitudes," Class. Quant. Grav., vol. 14, pp. 351-365, 1997. 183, 194

[185] L. J. Dixon and Y. Shadmi, "Testing gluon selfinteractions in three jet events at hadron colliders," Nucl. Phys., vol. B423, pp. 3-32, 1994. [Erratum: Nucl. Phys.B452,724(1995)]. 186

[186] S. He and Y. Zhang, "New Formulas for Amplitudes from HigherDimensional Operators," JHEP, vol. 02, p. 019, 2017. 186

[187] P. S. Norridge, "Recovering infinities in graviton scattering amplitudes using Cutkosky rules," Phys. Lett., vol. B387, pp. 701-706, 1996. 186

[188] H. J. Lu and C. A. Perez, "Massless one loop scalar three point integral and associated Clausen, Glaisher and L functions," 5 1992. 205, 206

[189] R. Ellis and G. Zanderighi, "Scalar one-loop integrals for QCD," JHEP, vol. 02 , p. 002, 2008. 205 